\title{
FERnerkundLICHe WALDFLÄCHEnERFASSUng IM KonteXt INTERNATIONALER UMWELTABKOMMEN
}

\author{
-Eine Analyse kritischer Faktoren-
}

\author{
DisSERTATION \\ zur Erlangung des Doktorgrades \\ der Fakultät für Forstwissenschaften und Waldökologie \\ der Georg-August-Universität Göttingen \\ vorgelegt von \\ Paul Magdon \\ geboren in Dresden
}

Göttingen, 2013 
1. Gutachter: Prof. Dr. Christoph Kleinn

2. Gutachter: Prof. Dr. Joachim Saborowski

Tag der mündlichen Prüfung: 19. April 2013 


\section{Danksagung}

Die Anfertigung dieser Arbeit wäre ohne die Unterstützung vieler Kollegen und Freunde nicht möglich gewesen. So gilt mein erster Dank Prof. Dr. Christoph Kleinn, der maßgeblich an der Entwicklung der dieser Arbeit zugrunde liegenden Ideen mitgewirkt und mich durch sein mir entgegengebrachtes Vertrauen in vielerlei Hinsicht ermutigt hat. Dr. Uwe Walz und Dr. Sebastian Hoechstetter standen mir bei der Berechnung der dreidimensionalen Landschaftsstrukturmaße hilfreich zur Seite. Die Feldarbeiten in Costa Rica entstanden in Zusammenarbeit mit Mauricio Arraya-Vega, dessen Familie mich in aller Gastfreundschaft in ihrem Hause aufnahm. Dr. David Morales stand mir mit organisatorischer Unterstützung bei den Feldaufnahmen in Guanacaste bei.

Gewinnbringend war der fachliche Austausch mit Christoph Fischer, Dr. Lutz Fehrmann, Dr. Hans Fuchs, Prof. Dr. Martin Schlather und Prof. Dr. Joachim Saborowski. Auch sei allen anderen Kollegen der Abteilung Waldinventur und Fernerkundung für die gute Arbeitsatmosphäre gedankt, im speziellen Hendrik Heydecke für die Kooperation bei der Programmierung des ForestEye-Prozessors, Rainhard Schlote für die wichtigen technischen und sportlichen Fachgespräche und Ulrike Docktor für ihre administrativen Kompetenzen.

Eine wichtige Grundvoraussetzung für die Arbeit war die finanzielle Förderung durch die Deutsche Forschungsgemeinschaft (DFG). Die RapidEye-Satellitenbilder stellte mir das Deutsche Zentrum für Luft und Raumfahrt (DLR) freundlich und komplikationslos aus dem RapidEye-Science-Archive zur Verfügung.

Für ihre liebevolle Geduld und ihr Verständnis, ihre moralische, orthografische und kulinarische Unterstützung danke ich meiner Frau Antonia Magdon aus vollem Herzen! 


\section{Zusammenfassung}

Als Reaktion auf die fortschreitende, anthropogen bedingte Veränderung und Zerstörung der Ökosysteme fand 1992 die Konferenz der Vereinten Nationen über Umwelt und Entwicklung in Rio de Janeiro statt. Die Konferenz markiert aus heutiger Sicht einen Startpunkt multilateraler Umweltschutzbemühungen, da offiziell formuliert wurde, dass die lokal auftretenden Umweltprobleme durch globale Veränderungen hervorgerufen werden und nur durch gemeinsame Bemühungen auf internationaler Ebene zu bewältigen sind. Ergebnis dieser Konferenz war die Verabschiedung verschiedener internationaler Abkommen zum Umweltschutz. Wälder spielen dabei in ihrer Funktion als Kohlenstoffspeicher und als terrestrischer Lebensraum mit der höchsten Artenvielfalt eine zentrale Rolle. Vor diesem Hintergrund stellen sich für die Waldinventur neue Aufgaben, sowohl bezüglich der zu erfassenden Zielgrößen als auch hinsichtlich der Rahmenbedingungen, da die erhobenen Informationen nicht mehr nur auf Betriebsebene für die Planung der Bewirtschaftung verwendet werden, sondern auch für die Erfüllung der internationalen Berichtspflichten. Als zentrale Größe des Waldmonitorings muss die Waldfläche gesehen werden, da sie die Grundlage für die meisten Berechnungen ist. Daneben wird die Waldfragmentierung, also die Form und räumliche Verteilung der Waldflächen, häufig als Indikator für die Biodiversität diskutiert.

Die fernerkundliche Erfassung der Waldfläche und die Beschreibung der Waldfragmentierung mit Landschaftsstrukturmaßen (LSM) im Kontext der internationalen Umweltabkommen ist Gegenstand der vorliegenden Arbeit. Zielsetzung ist es, kritische methodische und technische Aspekte, welche die Schätzung der Waldfläche und die Berechnung der LSM beeinflussen, zu identifizieren und ihre Wirkungen zu analysieren.

Anhand einer Literaturrecherche wurden zunächst vier kritische Faktoren identifiziert: i) die Walddefinition, ii) die Waldranddefinition, iii) der Beobachtungsmaßstab und iv) das Landschaftsmodell, welches für die Berechnung der LSM verwendet wird. Die Effekte und Wechselwirkungen zwischen den vier genannten Faktoren wurden im zweiten Teil der Arbeit auf Grundlage einer Simulationsstudie untersucht. Dafür wurden Kronenkarten und Geländemodelle auf Basis von Gauß'schen Zufallsfeldern in verschiedenen Auflösungstufen simuliert. Durch Variation der Mindestüberschirmung und der Größe der Referenzfläche, auf der die Überschirmung gemessen wird, konnten aus den Kronenkarten Waldkarten mit unterschiedlichen Wald- und Waldranddefinitionen erstellt werden. Zusätzlich wurde der Einfluss des Landschaftsmodells auf die Berechnung der LSM untersucht. Dies geschah mit 
Hilfe eines neuen Verfahrens, das die Berechnung der LSM im dreidimensionalen Raum ermöglicht. Die Ergebnisse der Simulation zeigen, dass alle vier Faktoren einen wesentlichen Einfluss auf die Waldflächenkarten haben können. Dabei ergeben sich besonders für die Kronenüberschirmung und die Referenzflächengröße spezifische Wechselwirkungen, die sich teilweise mit einem einfachen geometrischen Waldrandmodell theoretisch erklären lassen. So zeigt sich, dass besonders für Walddefinitionen mit einer Mindestüberschirmung, die stark von $50 \%$ abweicht, die Referenzflächengröße einen erheblichen Einfluss auf die Waldfläche und Fragmentierung hat.

Basierend auf den Ergebnissen der Simulationstudie wurde im 3. Teil der Arbeit ein Klassifikationschschema entwickelt, das es ermöglicht spezifische Kriterien einer Walddefinition in den Auswertungs- und Klassifikationsprozess von Fernerkundungsdaten zu integrieren, um standardisierte Waldkarten zu erstellen. Beispielhafte Grundlage war die Walddefinition der Food and Agriculture Organization of the United Nations (FAO), die Wald als eine Landnutzungsform beschreibt. Im Gegensatz zu Landbedeckungsformen können Landnutzungsklassen nicht direkt in Fernerkundungsdaten beobachtet werden. Zur Erstellung einer Landnutzungskarte müssen daher neben den Landbedeckungsklassen weitere Kontextinformationen berücksichtigt werden. Dafür wurde in der vorliegenden Arbeit ein hierarchischer Klassifikationsschlüssel entwickelt, der ausgehend von einer Landbedeckungskarte, eine Landnutzungs- und eine Waldkarte generiert. Die benötigten Kontextinformationen werden dabei mit Hilfe von Entscheidungsbäumen, die auf eine fixe Referenzfläche angewendet werden, berücksichtigt. Dieses Verfahren ermöglicht es, Waldkarten zu erstellen, die einer bestimmten vorher festgelegten Walddefinition entsprechen. Insofern kann das Verfahren zur Standardisierung der Waldflächenerfassung beitragen. Darüber hinaus bietet es die Möglichkeit die Walddefinition durch Änderung der Kriterien oder der Schwellenwerte flexibel anzupassen, sodass es als wissenschaftliches Werkzeug zur Analyse des Effektes verschiedener Walddefinitionen verwendet werden kann.

Im letzten Teil der Arbeit wurde eine Fallstudie durchgeführt, die untersucht inwieweit sich das entwickelte Verfahren operational für die Waldflächenerfassung einsetzen lässt. Da der Fokus der internationalen Umweltabkommen auf den tropischen Waldgebieten liegt, wurden für die Fallstudie zwei unterschiedliche tropische Waldlandschaften in Costa Rica ausgewählt. Zur Klassifikation der Landbedeckung kamen Satellitenbilder des RapidEye-Systems mit einer räumlichen Aufösung von $5 \mathrm{~m}$ zum Einsatz. Für die Klassifikation der Landbedeckung wurde zunächst eine Software entwickelt, welche atmosphärische und topographische Korrekturen, Bildverbesserung, nicht-parametrische Klassifikationsverfahren und den, 
im dritten Teil der Arbeit entwickelten hierarchischen Klassifikationsansatz für die Erstellung der Landnutzungskarten, implementiert. Die Ergebnisse der Fallstudie zeigen, dass das entwickelte Verfahren geeignet ist, Waldkarten für stark fragmentierte tropische Landschaften zu erstellen. Die Waldkarten entsprechen einer zuvor festgelegten Walddefinition (z. B. FAO), in der die einzelnen Kriterien (u. a. Mindestüberschirmung, Mindestgröße, vorherrschende Landnutzung) während der Klassifikation explizit geprüft werden.

Die vorliegende Arbeit zeigt theoretisch, empirisch und auch in der praktischen Anwendung, dass eine Vielzahl von Faktoren die Erfassung der Waldfläche beeinflusst. Einer der wichtigsten Faktoren ist dabei die Walddefinition. Die übliche Praxis bei der fernerkundlichen Erstellung von Waldkarten, die Klasse „Wald“ ohne expliziten Bezug auf geeignete Kriterien direkt auszuweisen, führt zu großen Unsicherheiten bei der Waldflächenschätzung und ist im Rahmen von international verbindlichen Abkommen kaum akzeptabel. Die Entscheidung welche Walddefinition verwendet werden soll, wird in politischen Verhandlungen bestimmt. Aufgabe der Waldinventur muss es dann sein, diese politischen Vorgaben umzusetzen. Die hier vorgestellten Methoden können insofern zur Standardisierung der fernerkundlichen Waldflächenerfassung beitragen, als das sie transparente Entscheidungsregeln implementieren und somit konsistente Waldkarten erzeugen. 


\section{Summary}

In reaction to the ongoing anthropogenetic changes of the earth' ecosystems the United Nations Conference on Environment and Development was hold in 1992 in Rio de Janeiro. This conference was an important starting point in environmental negotiations as it was recognized that locally observed problems are caused by global changes in the ecosystems and thus global actions are required. The conference resulted in the agreement on different international environmental conventions including the Convention on Biological Diversity (UN-CBD) and the Framework Convention on Climate Change (UN-FCCC) which in turn led to the Kyoto protocol. Forests play a significant role in both conventions either as being a carbon pool or as host to greater biodiversity. In this context forest monitoring faces new challenges regarding the target variables as well as the usage of the information which will no longer only be used for forest management decisions but also to fulfill international reporting obligations. The forest area and its fragmentation are the most fundamental variables of the required monitoring then.

The topic of this work is the remote sensing based assessment of forest area and characterization of its fragmentation by means of landscape metrics in the context of international environmental conventions with the specific objective to identify and analyze critical methodological and technical aspects and their relevance for forest cover monitoring systems.

Based on a literature review four critical factors were identified: i) forest definition, ii) forest edge definition, iii) scale of observation, and iv) the landscape model used to derive the landscape metrics. The effects and interactions of these factors were analyzed in the second part of the study using a spatial simulation based on artificial landscapes. Therefore tree crown maps and elevation models were simulated with different spatial resolutions using Gaussian random fields. By varying the minimum crown cover threshold and the reference area on which crown cover is determined, forest maps with different underling forest and forest edge definitions were generated. To analyze the effects of the landscape model on the calculation of landscape metrics a new approach which enables the calculation of landscape metrics in the three dimensional space was taken. The simulation results showed that all of the studied factors have an influence on the forest area estimates and the derived landscape metrics. Significant interactions between crown cover and the size of the reference area were 
found and could be explained partly by the analysis of a simple geometric forest edge model. A major finding of the simulation study is, that if the crown cover threshold is different from 0.5 , significant effects are to be expected from the size of the reference area.

Based on the findings from the simulation a classification framework was developed in the third part of the study which allows the integration of a specific forest and forest edge definition into the analysis of remote sensing data. The FAO forest definition is most widely used in the context of international environmental conventions and defines forest as a land use class. Land use can not be observed directly in remote sensing data without the integration of context information. Therefore a hierarchical classification key which allows the generation of land cover, land use and forest cover maps was developed. The required context information was collected by applying the concept of a fixed reference area. This approach allows the implementation of specific criteria of forest definitions and separates the process of image classification form the application of a forest definition. Thus forest definitions can easily be adopted to the user requirements and forest maps with different forest definition can be generated, which makes the framework an interesting research tool.

In the last part of the work the operational practicability of the developed approach was tested in a case study. Therefore two tropical highly fragmented forest landscapes in Costa Rica were selected. For both study sites land cover maps were generated from RapidEye satellite images. Therefore a software was programmed which implements the 6 -S atmospheric correction, topographic correction, image enhancement and the Random Forests classifier. The results of the case study demonstrated that the developed approach is capable of producing forest cover maps for highly fragmented tropical landscapes. The forest maps are in line with the current forest definitions (e.g. FAO) as the criteria (e.g. crown cover, minimum area, predominantly land use) are evaluated individually during the classification.

The study at hand shows theoretically, empirically and also practically that a number of factors influence the assessment of forest cover and fragmentation. The most important factor is the forest definition. The widespread practice to generate forest maps without the implication of specific forest definitions leads to large uncertainties of the forest area estimates which are hardly acceptable in the context of international conventions. The decision on criteria and thresholds of forest definitions are negotiated in a political debate. The forest 
inventory will be responsible to transcript the political guidelines into an operational monitoring system. The proposed approaches can contribute to the standardization of forest cover monitoring as they implement transparent definitions to generate consistent forest cover maps. 



\section{Inhaltsverzeichnis}

$\begin{array}{lll}\text { I Einleitung und Hintergrund } & 1\end{array}$

1 Wälder im Fokus internationaler Abkommen zum Schutz der Umwelt $\quad 3$

1.1 Der Weltgipfel von Rio de Janeiro . . . . . . . . . . . . . . . . . 3

1.2 Klimarahmenkonvention . . . . . . . . . . . . . . . . 4

1.2.1 Der Wald als Kohlenstoffspeicher . . . . . . . . . . . . . . 4

1.2.2 Politischer Hintergrund der Klimarahmenkonvention . . . . . . . . . . 5

1.2.3 Erfassung der Kohlenstoffbilanz von Wäldern . . . . . . . . . . . 5

1.3 Übereinkommen über die Biologische Vielfalt . . . . . . . . . . . . . . . . . 6

1.3.1 Der Wald als Habitat . . . . . . . . . . . . . . . 6

1.3.2 Politischer Rahmen des Übereinkommens über die Biologische Viel-

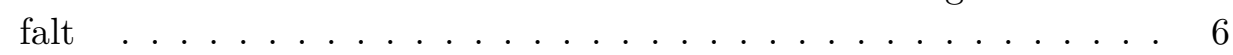

1.3.3 Erfassung der Biodiversität von Wäldern . . . . . . . . . . . . 7

1.4 Berichtspflichten der Rio Umweltabkommen . . . . . . . . . . . . . . . . 12

2 Erfassung der Waldfläche: Historische Entwicklung und Stand der Forschung 15

3 Kritische Faktoren der fernerkundlichen Waldflächenerfassung $\quad 21$

3.1 Walddefinition . . . . . . . . . . . . . . . 21

3.2 Waldranddefinition . . . . . . . . . . . . . . . . . . . 24

3.3 Landschaftsmodell . . . . . . . . . . . . . . . . . . . 25

3.4 Beobachtungsmaßstab . . . . . . . . . . . . . . . . 26

4 Zielsetzung und Gliederung der Arbeit $\quad 31$

II Sensitivitätsanalyse der kritischen Faktoren 33

5 Einleitung Simulation $\quad \mathbf{3 5}$

5.1 Ausgangssituation und Zielsetzung der Simulation . . . . . . . . . . . 35

5.2 Zielgrößen des Waldflächenmonitorings . . . . . . . . . . . . . . . 36

6 Simulationsmethoden $\quad 39$

6.1 Erstellung räumlicher Landschaftsmodelle . . . . . . . . . . . . . . . . . 39

6.2 Variation der Beobachtungsskalen . . . . . . . . . . . . . . . . . . 42

6.3 Waldflächenkartierung auf Basis von Überschirmungsgradienten . . . . . . . . 42

6.4 patch3D: Ein Programm zur dreidimensionalen Landschaftsanalyse . . . . . 44

6.5 Versuchsdesign . . . . . . . . . . . . . . . . 47 
7 Ergebnisse der Simulation $\quad 49$

7.1 Landschaftssimulation . . . . . . . . . . . . . . . . . . . . . . 49

7.2 Geometrische Analyse des Referenzflächenverfahrens . . . . . . . . . . . 50

7.3 Auswertung der Simulation . . . . . . . . . . . . . . . 52

7.3.1 Einfluss der Wald- und Waldranddefinition . . . . . . . . . . . . 52

7.3.2 Einfluss des Landschaftsmodells . . . . . . . . . . . . . . . . . . 56

7.3 .3 Skalen-Effekte . . . . . . . . . . . . . . . . . . . . . . 59

8 Diskussion der Simulationsergebnisse $\quad 63$

9 Schlussfolgerungen für die Standardisierung der Waldflächenerfassung $\quad 71$

III Entwicklung eines Verfahrens zur Standardisierung der fernerkundlichen Waldflächenerfassung

10 Integration einer spezifischen Walddefinition in die Auswertung von Ferner$\begin{array}{ll}\text { kundungsdaten } & \mathbf{7 5}\end{array}$

11 Der ForestEye-Prozessor $\quad \mathbf{7 9}$

11.1 Struktur und Aufbau . . . . . . . . . . . . . . . . . . . . 79

11.2 Bildkalibrierung . . . . . . . . . . . . . . . . . . . 80

11.2.1 Atmosphärenkorrektur . . . . . . . . . . . . . . 80

11.2.2 Topographische Korrektur . . . . . . . . . . . . . . . . . . . 84

11.3 Bildverbesserung . . . . . . . . . . . . . . . . . . 86

11.3.1 Vegetationsindices . . . . . . . . . . . . . 86

11.3 .2 Texturindices . . . . . . . . . . . . . . . . 86

11.4 Random Forests Klassifikation _. . . . . . . . . . . . . . . . . 90

11.5 Evaluation der Klassifikationsergebnisse . . . . . . . . . . . . . . . 94

$\begin{array}{ll}\text { IV Fallstudie: Costa Rica } & 97\end{array}$

$\begin{array}{lr}12 \text { Ausgangssituation } & 99\end{array}$

13 Untersuchungsgebiete und Bildmaterial $\quad 101$

13.1 Beschreibung der Untersuchungsgebiete . . . . . . . . . . . . . . . . . 101

13.2 Bildmaterial . . . . . . . . . . . . . . . . . . . . . 104

13.2 .1 RapidEye . . . . . . . . . . . . . . . . . . . . . 104

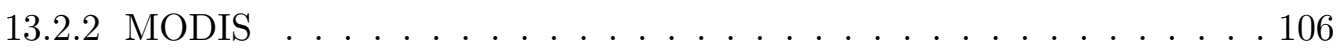

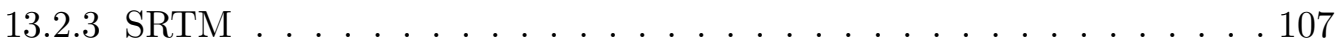

14 Erfassung der Referenzdaten $\quad 109$

14.1 Trainings- und Validierungsdaten aus der Bildinterpretation . . . . . . . . . 109 
14.2 Validierungsdaten aus terrestrischen Aufnahmen . . . . . . . . . . . . 110

15 Ergebnisse der Fallstudie 113

15.1 Bildkalibrierung . . . . . . . . . . . . . . . . . . . 113

15.1.1 Atmosphärenkorrektur . . . . . . . . . . . . . . . . . 113

15.1.2 Georeferenzierung . . . . . . . . . . . . . . . 116

15.1.3 Korrektur der reliefbedingten Beleuchtungsunterschiede . . . . . . . 118

15.2 Analyse der RF-Modelle . . . . . . . . . . . . . . . . . . . . . . . . . . . 119

15.2.1 Parametrisierung der RF-Modelle . . . . . . . . . . . . . . . 119

15.2.2 Variablenselektion . . . . . . . . . . . . . . . . . . 121

15.2.3 Güte der RF-Modelle . . . . . . . . . . . . . . . . . . . . . 121

15.3 Evaluation der Landbedeckungskarten . . . . . . . . . . . . . . . . 123

15.4 Vergleich der terrestrisch und fernerkundlich gemessenen Kronenüberschirmung . . . . . . . . . . . . . . . . . . . . . . . . . . . . . . . . . .

16 Diskussion der Fallstudie $\quad 133$

16.1 Einleitung . . . . . . . . . . . . . . . . . . . . . . 133

16.2 Umsetzung der Kriterien aus der FAO-Walddefinition . . . . . . . . . . . 133

16.3 Erstellung der Waldkarten mit dem ForestEye-Prozessor . . . . . . . . . . 136

16.3.1 Eignung der RapidEye-Bilder . . . . . . . . . . . . . . . 136

16.3 .2 Bildkalibrierung. . . . . . . . . . . . . . . . . . 136

16.3.3 Random Forests Klassifikator . . . . . . . . . . . . . . . . . 138

16.3.4 Qualität der Karten . . . . . . . . . . . . . . . . . . . . 139

16.4 Kartierung und Schätzung der Waldfläche im Rahmen internationaler Umweltabkommen . . . . . . . . . . . . . . . . . 143

$\begin{array}{lll}\text { V Schlußbetrachtung } & 147\end{array}$

17 Möglichkeiten und Grenzen der fernerkundlichen Waldflächenerfassung $\quad 149$

$\begin{array}{ll}\text { VI Literatur } & 153\end{array}$

$\begin{array}{ll}\text { VII Anhang } & 173\end{array}$ 



\section{Abbildungsverzeichnis}

1 Grundtypen der simulierten Baumkronenkarten . . . . . . . . . . . . . . . . . 41

2 Ablaufplan zur Anwendung des Referenzflächenverfahrens . . . . . . . . . . . 43

3 Berechnung der dreidimensionalen Landschaftsstrukturmaße . . . . . . . . . . 45

4 Schematische Darstellung des Waldrandmodells . . . . . . . . . . . . . . . . 50

5 Schema der Waldflächenkartierung . . . . . . . . . . . . . . . . . 52

6 Box-Whisker Plots der Waldanteile . . . . . . . . . . . . . . . . 53

7 Box-Whisker Plots der Patchanzahl . . . . . . . . . . . . . . . . 55

8 Box-Whisker Plots der mittleren Patchgröße . . . . . . . . . . . . . 56

9 Box-Whisker Plots der Landschaftsstrukturmaße . . . . . . . . . . . . 57

10 Gegenüberstellung der zwei- und dreidimensionalen Landschaftsstrukturmaße 58

11 Landschaftsausschnitt in drei verschiedenen horizontalen Auflösungen . . . . 59

12 Gegenüberstellung der Landschaftsstrukturmaße zweier horizontaler Auflö-

sungen . . . . . . . . . . . . . . . . . . . 6 61

13 Gegenüberstellung der Landschaftsstrukturmaße zweier vertikaler Auflösungen 61

14 Klassifikationsschlüssel der FAO-Landnutzungsklassen . . . . . . . . . . . . . 77

15 Entscheidungsbaum zur Überführung der Level-I in Level-II Klassen . . . . . 78

16 Aufbau des ForestEye-Prozessors . . . . . . . . . . . . . . . . . . . . . 79

17 Strahlungswege zwischen Sonne, Erde und Sensor . . . . . . . . . . . . . . . . 81

18 Beispielhafter Ablauf zur atmosphärischen Korrektur von RapidEye-Daten . 84

19 Schema zur Erstellung der Grauwertübergangsmatrix . . . . . . . . . . . . . . 89

20 Schematische Darstellung eines Entscheidungsbaums . . . . . . . . . . . . . . 92

21 Lage der Untersuchungsgebiete . . . . . . . . . . . . . . . . . . . . . . 101

22 Typisches Landschaftsbild in Osa . . . . . . . . . . . . . . . . . . 102

23 Typisches Landschafstbild in Guanacaste . . . . . . . . . . . . . . . . . . 103

24 Klimadiagramme der Untersuchungsgebiete . . . . . . . . . . . . . . . . . . . 104

25 Probeflächendesign der terrestrischen Aufnahmen . . . . . . . . . . . . . . . 111

26 Verteilung der Pixelwerte vor und nach der atmosphärischen Korrektur . . . . 115

27 NDVI-Werte entlang eines Transektes vor und nach der atmosphärischen Kalibrierung . . . . . . . . . . . . . . . . . . . . 116

28 NIR-Reflexionswerte entlang eines Transektes vor und nach der topographischen Korrektur . . . . . . . . . . . . . . . . . . . . . . . . . 118

29 Modellfehler für die unterschiedliche Anzahl von Entscheidungsbäumen und Variablen . . . . . . . . . . . . . . . . . . . . . 120

30 Z-Scores der 25 Bänder . . . . . . . . . . . . . . . . . . 122

31 Klassifikationsergebnisse Guanacaste . . . . . . . . . . . . . . . . . . . 127

32 Klassifikationsergebnisse Osa . . . . . . . . . . . . . . . . . 128

33 Verschiedene Situationen bei der terrestrischen Aufnahme der Linienstichproben . . . . . . . . . . . . . . . . . . . . . . 130

34 Vergleich der terrestrisch und fernerkundlich bestimmten Kronenüberschirmung 131 
35 Veränderung der Patchform für verschiedene Walddefinitionen . . . . . . . . . 175

36 Feldkarte für Stichprobenpunkt 141 . . . . . . . . . . . . . . . . . . 176 


\section{Tabellenverzeichnis}

1 Untersuchte Landschaftsstrukturmaße . . . . . . . . . . . . . . . . . 38

2 Faktoren und Faktorenstufen der Simulation . . . . . . . . . . . . . . . . 47

3 Kenngrößen der simulierten Landschaften . . . . . . . . . . . . . . . . . . . 49

4 Differenzen der zwei- und dreidimensionalen Waldkarten . . . . . . . . . . . 57

5 Differenzen der Waldkarten mit verschiedenen horizontalen Auflösungen . . . 60

6 FAO-Landnutzungsklassen . . . . . . . . . . . . . . . 76

7 Vegetationsindices . . . . . . . . . . . . . . . . 87

8 Texturindices erster Ordnung . . . . . . . . . . . . . . . 88

9 Texturindices zweiter Ordnung . . . . . . . . . . . . . . . . . . . 90

10 Spektrale Aufösung der RapidEye-Sensoren . . . . . . . . . . . . . . . . . . . 105

11 RapidEye-Aufnahmeparameter . . . . . . . . . . . . . . . . . . 106

12 Für die Atmosphärenkorrektur verwendete MODIS-Produkte. . . . . . . . . . 107

13 Geplante Verteilung der Probepunkte auf die vier Straten . . . . . . . . . . . 110

14 Aus den MODIS-Daten berechnete Atmosphärenparameter . . . . . . . . . . 114

15 Kontrollpunktanzahl der Georeferenzierung . . . . . . . . . . . . . . . 117

16 Modellfehler der verwendeten RF-Modelle . . . . . . . . . . . . . . . . . . . 123

17 Fehlermatrix Osa . . . . . . . . . . . . . . . . . . . . 125

18 Fehlermatrix Guanacaste . . . . . . . . . . . . . . . . . 126

19 Waldflächenschätzung beider Untersuchungsgebiete . . . . . . . . . . . . . . 129

20 Verteilung der Probepunkte auf die vier Straten . . . . . . . . . . . . . . . 130 

Teil I.

Einleitung und Hintergrund 



\section{Wälder im Fokus internationaler Abkommen zum Schutz der Umwelt}

\subsection{Der Weltgipfel von Rio de Janeiro}

1992 fand in Rio de Janeiro die Konferenz der Vereinten Nationen über Umwelt und Entwicklung (United Nations Conference on Environment and Development, UNCED) statt. Sie war eine Reaktion auf die fortgesetzten, anthropogen bedingten Veränderungen und Zerstörungen der Ökosysteme, von denen das Wohlergehen der Menschheit abhängt. Ihre Bedeutung erhält die Konferenz aus heutiger Sicht, da die Teilnehmer hier erstmals offiziell darin übereinstimmten, dass die lokal auftretenden Umweltprobleme durch globale Veränderungen hervorgerufen werden und daher nur durch gemeinsame Bemühungen auf internationaler Ebene bewältigt werden können. Auf Grundlage dieser Erkenntnis wurden Entwicklungs- und Umweltziele für das 21. Jahrhundert in der Agenda 21 festgelegt und von 178 Nationen ratifiziert.

Die formulierten Umweltziele beinhalten unter anderem den Erhalt der biologischen Vielfalt, die Bekämpfung der Entwaldung, den Schutz der Erdatmosphäre und die Bekämpfung von Wüstenbildung und Dürre. Um die gesetzten Ziele zu erreichen, wurden drei Übereinkommen/Konventionen verabschiedet: i) die Klimarahmenkonvention (United Nations Framework Convention on Climate Change, UNFCCC), ii) das Übereinkommen über die Biologische Vielfalt (United Nations Convention on Biodiversity, UNCBD) und iii) das Übereinkommen der Vereinten Nationen zur Bekämpfung der Wüstenbildung in den von Dürre und/oder Wüstenbildung schwer betroffenen Ländern, insbesondere in Afrika (United Nations Convention to Combat Desertification in those Countries Experiencing Serious Drought and/or Desertification, particularly in Africa, UNCCD). Auch wenn kein gesondertes Abkommen über den Erhalt oder den Schutz von Wäldern verabschiedet wurde, so sind verschiedene Waldfunktionen besonders für das UNFCCC- und das UNCBD-Abkommen von zentraler Bedeutung. 


\subsection{Klimarahmenkonvention}

\subsubsection{Der Wald als Kohlenstoffspeicher}

Durch Respiration und Assimilation kommt es zu einem Austausch von Kohlenstoff zwischen der Atmosphäre und der lebenden Biomasse von Wäldern. In Abhängigkeit von der Bilanz dieses Austausches können Wälder sowohl Quellen als auch Senken für atmosphärischen Kohlenstoff darstellen. Die Kohlenstoffbilanz des Waldes wird im wesentlichen von seiner Produktivität bestimmt. Wälder, die sich in der Aufbauphase befinden, akkumulieren Kohlenstoff in Form von Biomasse bis sie einen stabilen Zustand erreichen, bei dem Respiration und Assimilation im Gleichgewicht sind. Wälder, die in ihrer Entwicklung nicht durch anthropogene Eingriffe beeinflusst werden, erreichen diesen stabilen Zustand nach einigen wenigen Baumgenerationen. So befinden sich die meisten tropischen Primärwälder in einem solchen Gleichgewicht. In den gemäßigten Breiten werden die meisten Wälder mit dem Ziel einer möglichst hohen Holzproduktion bewirtschaftet. Zur Steigerung der Holzproduktion wird der Wald durch die gezielte Entnahme von Biomasse permanent in der Aufbauphase gehalten. Daher stellen diese nachhaltig bewirtschafteten Wälder häufig eine Kohlenstoffsenke dar. Dem steht die Umwandlung von Waldflächen in andere Landnutzungsformen gegenüber. Besonders in den tropischen Waldgebieten führt dies dazu, dass große Mengen Kohlenstoff freigesetzt werden.

Im globalen Kohlenstoffkreislauf spielen Wälder eine wichtige Rolle, da sie etwa ein Viertel der gesamten Landfläche bedecken und 90\% der lebenden terrestrischen Biomasse speichern (Fabian, 2002). In einer aktuellen Veröffentlichung schätzen Pan et al. (2011), dass Wälder weltweit $861 \pm 66 \mathrm{Pg}$ Kohlenstoff speichern. Davon sind $383 \pm 30 \mathrm{Pg}$ (44\%) im Boden, $363 \pm 28 \mathrm{Pg}(42 \%)$ in der lebenden Biomasse, $73 \pm 6 \mathrm{Pg}(18 \%)$ im Totholz und $43 \pm 3 \mathrm{Pg}$ (5\%) in der Bodenstreu. Die Hälfte des in Wäldern gespeicherten Kohlenstoffs befindet sich in tropischen Gebieten. Durch die Umwandlung dieser Wälder werden jährlich $2.9 \pm 0.5 \mathrm{Pg}$ Kohlenstoff freigesetzt, von denen 1.6 $\pm 0.5 \mathrm{Pg}$ durch die Wiederbewaldung mit tropischen Sekundärwäldern aufgenommen werden. Insgesamt werden also $1.3 \pm 0.7 \mathrm{Pg}$ Kohlenstoff durch die Umwandlung von tropischen Wäldern jährlich freigesetzt. Da die gemäßigten Wälder zwischen 1990 und 2007 insgesamt 2.4 \pm 0.4Pg Kohlenstoff aufgenommen haben, schlussfolgern die Autoren, dass Wälder insgesamt eine Kohlenstoffsenke von $1.1 \pm 0.8 \mathrm{Pg}$ darstellen. 


\subsubsection{Politischer Hintergrund der Klimarahmenkonvention}

Die Teilnehmer des Weltgipfels von Rio 1992 stellten in einer gemeinsamen Erklärung fest, dass die anthropogen bedingte Veränderung des globalen Klimas eine der größten Herausforderungen für den Erhalt einer lebensfreundlichen Umwelt ist. Daher wurde die Klimarahmenkonvention beschlossen, in der sich die Unterzeichnerstaaten verpflichten ihre Treibhausgasemissionen zu reduzieren. Die Reduktionsmengen und Berechnungsvorschriften wurden im Kyoto-Protokoll am 11.12. 1997 erstmals festgelegt. Zwei Artikel des Protokolls berücksichtigen Wälder als Kohlenstoffspeicher:

- Art. 3.3 legt fest, dass die Annex-I Staaten ihre Treibhausgasemissionen aus Landnutzungsänderungen bilanzieren müssen.

- Art. 3.4 sieht vor, dass die Bewirtschaftung von Wäldern als Kohlenstoffsenke angerechnet werden kann.

In einer Untersuchung über die Folgekosten des Klimawandels auf Basis eines ökonomischen Vergleiches der verschiedenen Optionen zur Reduktion von Treibhausgasemissionen schlussfolgerte Stern (2007), dass es am wirtschaftlichsten sei die Entwaldungsraten zu reduzieren. Motiviert durch diesen Report rückte der Wald als Kohlenstoffspeicher weiter in den Fokus der Klimawandeldebatte. Dies gab den Anstoß für ein Programm zur Reduktion von Treibhausgasemissionen durch Entwaldung und Walddegradierung (Reduced Emissions from Deforestation and Degradation, REDD). Auf der 16. Konferenz der Vertragsparteien (Conference of the Parties, COP) in Mexiko beschlossen die Vertragsstaaten, dass zukünftig die Kohlenstoffsenken aus dem REDD Programm bei der Berechnung des nationalen Treibhausgasinventars berücksichtigt werden dürfen.

\subsubsection{Erfassung der Kohlenstoffbilanz von Wäldern}

Die Kohlenstoffbilanz eines Waldes kann mit verschiedenen Methoden bestimmt werden: i) durch Schätzung der Netto-Primär-Produktion ii) durch Eddy-Kovarianz-Messungen oder iii) durch Schätzung der Kohlenstoffvorräte mit Waldinventuren zu unterschiedlichen Zeitpunkten. Die Methoden für die Bilanzierung der Kohlenstoffflüsse innerhalb der Klimarahmenkonvention werden vom Zwischenstaatlichen Ausschuss für Klimaänderungen (Intergovernmental Panel on Climate Change, IPCC) entwickelt. Dieser hat festgelegt, dass die C-Vorräte $\left(C_{\text {total }}\right)$ nach Gleichung 1 durch Multiplikation der Waldfläche $A$ (activity data) 
mit einer entsprechenden Kohlenstoffdichte $E F$ (emission factor) berechnet werden können (UNFCCC, 2003).

$$
C_{\text {total }}=A \times E F
$$

Für die Ermittlung der Kohlenstoffdichte hat IPCC drei Methoden festgelegt, die sich durch ihre Komplexität und Anforderungen an die Daten unterscheiden: Tier 1 verwendet global vorgegebene Emissionsfaktoren, Tier 2 erfordert die Aufnahme lokaler Vorräte und Tier 3 verlangt die wiederholte Aufnahme von permanenten Probeflächen. Für die Erfassung der Waldfläche werden bisher keine konkreten Methoden vorgeschrieben. Einziger Anhaltspunkt ist eine Walddefinition, mit einigen festgelegten Kriterien. Dies führt im Vergleich zu anderen Emittenten von Treibhausgasen zu großen Unsicherheiten bei der Quantifizierung.

\section{3. Übereinkommen über die Biologische Vielfalt}

\subsubsection{Der Wald als Habitat}

Der Wald ist das terrestrische Ökosystem mit den höchsten Artenzahlen weltweit. Der Schutz dieser Lebensräume ist ein wichtiges Element des Übereinkommens über die Biologische Vielfalt (UNCED,1992, Art. 8d). Durch die Intensivierung und Industrialisierung der Landwirtschaft kommt es weltweit zu starken Veränderungen der Lebensräume, die im Bereich der Wälder entweder zu einer Reduktion der Waldfläche oder zu einer Fragmentierung führen. Letztere ist neben der Zerstörung der tropischen Wälder die größte Gefahr für die tropische Biodiversität (Laurance \& Peres, 2006).

\subsubsection{Politischer Rahmen des Übereinkommens über die Biologische Vielfalt}

Neben dem Klimawandel ist die Abnahme der biologischen Vielfalt eine wesentliche Bedrohung für das Leben auf der Erde. Daher wurde auf der Konferenz von Rio ein zweites Abkommen, das Übereinkommen über die Biologische Vielfalt, verabschiedet. Im Rahmen des Übereinkommens verpflichten sich die Vertragsstaaten unter anderem die biologische Vielfalt zu erhalten, deren Bestandteile nachhaltig zu nutzen und den Vorteil, der sich aus 
der Nutzung der genetischen Ressourcen ergibt, gerecht zu verteilen (UN, 1992). Ein wichtiger Punkt des Übereinkommens ist die Überwachung bestimmter Teile der biologischen Vielfalt, die in Art. 7 festgelegt ist. Eine genauere Beschreibung wie diese Überwachung durchzuführen ist, findet sich in dem Übereinkommen nicht. Es wird lediglich spezifiziert, dass „durch Probenahme und andere Verfahren ... Bestandteile der biologischen Vielfalt überwacht ..." (UN, 1992) werden sollen. Der neue strategische Plan des Übereinkommens formuliert für den Zeitraum von 2011-2020 zwanzig Ziele (Aichi-biodiversity targets). Dabei sieht das zweite Ziel vor, dass bis spätestens 2020 „Biodiversitätswerte“ in einer Nationalinventur erfasst und protokolliert werden.

\subsubsection{Erfassung der Biodiversität von Wäldern}

Das Übereinkommen über die Biologische Vielfalt definiert Biodiversität wie folgt:

„Biologische Vielfalt bedeutet die Variabilität unter lebenden Organismen jeglicher Herkunft, darunter unter anderem Land-, Meeres- und sonstige aquatische Ökosysteme und die ökologischen Komplexe, zu denen sie gehören; dies umfasst die Vielfalt innerhalb der Arten und zwischen den Arten und die Vielfalt der Ökosysteme." (UN, 1992)

Dieser weit gefasste Biodiversitätsbegriff beschreibt die Vielfalt auf vier Skalenebenen: i) der genetische Ebene ii) der Arten- oder Populationsebene iii) der Ökosystemebene und iv) der Landschaftsebene. Zur Erfassung der Biodiversität müssen entsprechend der Skalen verschiedene Monitoringansätze gewählt werden. Die gleichzeitige Erfassung der Biodiversität auf allen Skalenebenen ist nur im Rahmen wissenschaftlicher Untersuchungen möglich, bei denen sehr kleine Landschaftsausschnitte intensiv beprobt werden. Im Rahmen von nationalen Inventuren, wie sie im strategischen Plan des CBD vorgesehen sind, müssen daher Indikatoren verwendet werden. Indikatoren stellen in diesem Zusammenhang Größen dar, die einfach zu erfassen sind und Rückschlüsse auf verschiedene Aspekten der Biodiversität ermöglichen. In Reaktion auf die Entscheidung VII/30 der COP der UNCBD wurde eine Initiative zur Entwicklung geeigneter Biodiversitätsindikatoren, die Biodiversity Indicator Partnership (BIP), gegründet. In einer im "Science Magazin" veröffentlichten Studie des BIP (Butchart et al., 2010) werden die entwickelten Indikatoren und ihr Trend über die letzten Dekaden vorgestellt. Zwei dieser Indikatoren beziehen sich direkt auf das Ökosystem Wald: die Waldfläche (forest extent) und die Waldfragmentierung (forest fragmentation). 
Beide vorgeschlagenen Indikatoren setzen auf der Landschaftsebene an, wobei die Waldfläche die Ausdehnung oder den Anteil der Wälder an der Landschaft und die Waldfragmentierung die Größe, Form und Anordnung der Waldflächen in der Landschaft beschreibt. Li \& Wu (2004) erläutern die Notwendigkeit, zunächst eine Beziehung zwischen der Landschaftsstruktur und den zu untersuchenden Prozessen nachzuweisen. Geschieht dies nicht, ist die Auswertung der Landschaftsstruktur für die gegebene Fragestellung nicht zielführend und kann zu falschen Schlussfolgerungen führen. Daher soll im folgenden Abschnitt geklärt werden, welche biologischen Prozesse Einfluss auf die Biodiversität eines Gebietes haben.

Ein weit verbreitetes Modell, das Erklärungen für die Artenanzahl in einem abgegrenzten Gebiet gibt, ist die Equilibrium-Theorie, die von MacArthur \& Wilson (1967) beschrieben wurde und als zentrale Theorie der Biogeographie und Ökologie von Lebensgemeinschaften gesehen werden muss. MacArthur \& Wilson (1967) untersuchten die Artenzahlen auf Inseln und formalisierten die Abhängigkeit dieser von der Größe einer Insel und ihrer Entfernung zum Festland. Sie verknüpften zwei ökologische Prozesse: die Migration und die Extinktion von Arten. Dabei hängt die Migration von der Entfernung oder Isolation und die Extinktion von der Größe der Insel ab. Das sich einstellende Gleichgewicht aus beiden Prozessen legt die Artenanzahl auf einer Insel fest. Sie entwickelten ihre Idee für Inseln, da sie sich leicht von ihrer Umgebung - dem Meer - abgrenzen ließen. Dabei stellten die Inseln einen geeigneten und das Meer einen ungeeigneten Lebensraum dar. Der Kontinent/das Festland ist der Ausgangspunkt für die Besiedlung der Inseln. Dieses, oft auch als „Inselmodell“ bezeichnete Modell, änderte das ökologische Denken, indem es die Anordnung der Habitate im Raum als einen wichtigen Einfluss auf die Populationen beschreibt (Harrison \& Bruna, 1999). Aus der Equilibrium-Theorie ergeben sich Schlussfolgerungen für die Artenvielfalt:

1. Viele Inseln bieten unterschiedliche Lebensräume und führen dadurch zu höherer Artenvielfalt.

2. Größere Inseln begünstigen höhere Artenzahlen durch niedrige Extinktionsraten.

3. Kürzere Entfernungen zwischen den Inseln erhöhen die Migrationsraten und führen zu einer höheren Artenanzahl.

4. Die Form der Insel beeinflusst die Küstenlänge und damit die Randeffekte, die sich negativ auf die Habitatqualität auswirken können.

Um dieses, von der Populationsdynamik geprägte, Modell auf die Problematik der Waldfragmentierung und den damit verbundenen Rückgang der Artenvielfalt zu übertragen, muss zunächst das Insel- auf ein Landschaftsmodell übertragen werden. Im weitesten Sinne kann 
eine Landschaft als ein heterogenes räumliches Mosaik verschiedener miteinander interagierender Elemente betrachtet werden, das sich üblicherweise über mehrere Kilometer erstreckt. Zur quantitativen Beschreibung der Landschaftsstruktur entwickelte Forman (1995b) das Patch-Corridor-Matrix (PCM)-Modell. Es unterscheidet drei Typen von Landschaftselementen:

\section{- Patch}

Ein Patch stellt eine von anderen abgegrenzte Fläche mit homogener Landbedeckung dar (Forman, 1995a). Die Kriterien, nach denen ein Patch ausgewiesen werden kann, sind hauptsächlich vom Gegenstand der jeweiligen Untersuchung geprägt. Übertragen auf Artverbreitungsmodelle wird meist angenommen, dass ein Patch ein geeignetes Habitat darstellt.

\section{- Korridor}

Korridore können als spezieller Fall von Patches betrachtet werden. Sie sind lineare Landschaftselemente, die Patches miteinander verbinden und somit einen Austausch von Energie, Material, etc. zwischen diesen ermöglichen.

- Matrix

Die Matrix ist der dominante Landbedeckungstyp in Bezug auf die Ausdehnung, Konnektivität und Kontinuität innerhalb der Landschaft (Forman, 1995a) und stellt typischerweise den Hintergrund dar. In Artverbreitungsmodellen wird meist angenommen, dass die Matrix nicht als Habitat geeignet ist.

Die Landschaftsstruktur ergibt sich aus Größe, Anzahl, Typ und Anordnung dieser drei Landschaftselemente (Leitão et al., 2006). Bei der Beschreibung der Struktur können zwei Aspekte unterschieden werden: die Landschaftskomposition erfasst die Anzahl und den Anteil der verschiedenen Patchtypen an der Landschaft. Die Landschaftskonfiguration erfasst die räumliche Anordnung, also die Position und Ausrichtung der verschiedenen Landschaftselemente. Die Landschaftsstrukturmaße (LSM) charakterisieren sowohl die Konfiguration als auch die Komposition in Form von quantitativen Metriken. In den letzten 20 Jahren wurden unzählige LSM für sehr verschiedene Fragestellungen entwickelt und angewendet. Ein guter Indikator für die wachsende Anzahl der LSM ist die Entwicklung des weit verbreiteten Softwarepaketes FRAGSTATS (McGarigal et al., 2002). Die aktuelle Version 4.1 stellt bereits über 100 LSM zur Verfügung. 
Überträgt man das Inselmodell auf das $P C M$-Landschaftsmodell können die Waldflächen (Patches) als Inseln und die Nicht-Waldflächen (Matrix) als Meere interpretiert werden. Der Prozess der Waldfragmentierung führt zu einer Verringerung der mittleren Patchgröße, einer Erhöhung der Anzahl an Patches und einer Zunahme der Randlänge (Rutledge, 2003). Dabei finden drei Veränderungen statt (Kupfer et al., 2006):

1. Reduktion der Waldfläche

Folgt man der Equilibrium-Theorie führt die Reduktion der Habitatfläche zu einer geringeren Bandbreite an Habitaten und zu einer Verringerung der vorhandenen Ressourcen. Ersteres hat eine direkte Reduktion der Artenanzahl zur Folge. Letzteres führt aufgrund des geringeren Angebotes an Ressourcen zu kleineren, stärker vom Aussterben bedrohten Populationen. Der ökologische Prozess der Extinktion ist auf die evolutionäre Anpassung und Spezialisierung der Arten an ein bestimmtes Habitat zurückzuführen. Beim Verlust dieses Habitates kommt es in Abhängigkeit des Spezialisierungsgrades der Art zur Extinktion.

2. Isolierung der Waldflächen

Die Struktur der Landschaft hat Auswirkungen auf die Besiedlung, die Bewegung und den genetischen Austausch der Arten. Eine erhöhte Isolation führt zu geringeren Besiedlungen, weniger genetischem Austausch und eingeschränkter Mobilität. Folgt man der Equilibrium-Theorie führt dies ebenfalls zur Reduktion der Artenanzahl.

3. Verlängerung des Waldrandes

Durch die Teilung der Waldflächen erhöht sich der Anteil an Waldrändern, die sich in Mikroklima, Struktur und Zusammensetzung wesentlich von den inneren Bereichen der Wälder unterscheiden. Saunders et al. (1991) geben eine gute Übersicht über die abiotischen und biotischen Veränderungen, die durch die Randeffekte hervorgerufen werden können.

Die ersten beiden Effekte können leicht als Biodiversitätsindikatoren interpretiert werden. Eine Verringerung der Patchgröße müsste eine Abnahme der Artenanzahl zur Folge haben und eine Erhöhung der Patchanzahl die Isolation der Waldflächen erhöhen, was ebenfalls eine Abnahme der Artenanzahl erwarten ließe. Der dritte Effekt, Verlängerung des Waldrandes, ist in dem Inselmodell nicht berücksichtigt. Er beschreibt die Veränderung der Habitate 
an ihren Rändern, deren Wirkung nicht unmittelbar klar ist und im Einzelfall untersucht werden muss.

Eine Vielzahl von Studien prüfte die Übertragbarkeit der Equilibrium-Theorie und der dahinter stehenden biologischen Modelle auf Waldlandschaften. Eines der frühesten und bis heute umfangreichstes Projekt, welches Auswirkungen der Waldfragmentierung in tropischen Regenwäldern untersuchte, ist das Biological Dynamics of Forest Fragments Project (BDFFP). Es wurde in den 70er Jahren durch T. Lovejoy begründet und hat seitdem sehr viele Publikationen hervorgebracht (Laurance et al., 2010). In der Zusammenfassung der wesentlichen Ergebnisse dieser Studien von Laurance et al. (2010) werden folgende Effekte beschrieben:

- Eine Abnahme der Artenanzahl in Abhängigkeit von der Größe der Fragmente konnte unter anderem für Bryophyten, Baumsamen, Palmen, insektenverzehrende Vögel im Unterwuchs, Primaten und größere herbivore Säugetiere beschrieben werden.

- Durch die Verlängerung der Waldränder kommt es zur Änderung im hydrologischen Gefüge der Regenwälder, die bis zu $2.7 \mathrm{~km}$ weit in den Wald reichen.

- An den neuen Waldrändern kommt es zu Austrocknungen und Veränderungen des Windregimes.

- Die Abundanz von Bienen, Wespen, Fliegen, Käfern, Ameisen, Schmetterlingen und Vögeln im Unterwuchs nimmt an den Waldrändern ab.

- Der Randeffekt summiert sich insofern, als dass das Auftreten mehrerer Ränder in unmittelbarer Nachbarschaft die Randeffekte verstärkt.

- Die Randeffekte hängen von der ausgebildeten Struktur des Randes ab und verändern sich mit der Zeit so, dass sie abgeschwächt werden.

- Eine Entfernung von $200 \mathrm{~m}$ zwischen zwei Patches stellt für viele Arten eine unüberwindbare Barriere dar.

- Die Struktur der Matrix zwischen den Patches kann Randeffekte verstärken oder mildern.

- Durch das, vom Trockenstress bedingte, Absterben alter Bäume am Waldrand führt die Fragmentierung zu einer Senkung der Kohlenstoffdichte.

- Der Rückgang der Artenvielfalt durch die fortgesetzte Fragmentierung ist systematisch und lässt sich für viele Gruppen beschreiben.

Harrison \& Bruna (1999) geben einen Überblick über eine Vielzahl von Studien, welche die biologischen Effekte der Fragmentierung analysieren. Sie schlussfolgern, dass durch die 
Fragmentierung eine biologische Verarmung der verbleibenden Flächen stattfindet. Dies drückt sich z. B. im Rückgang von spezialisierten Arten und im Anstieg von Generalisten aus. Marshall et al. (2010) untersuchen die Anzahl der Affenarten in einem Waldgebiet in Tanzania. Auch sie zeigen eine klare Abhängigkeit zwischen der Größe eines Waldpatches und der Anzahl der Arten.

\subsection{Berichtspflichten der Rio Umweltabkommen}

Aus der Klimarahmenkonvention ergeben sich innerhalb der nationalen Treibhausgasinventur Berichtspflichten, die es erfordern, Treibhausgasemissionen, welche durch Landnutzungsänderungen verursacht werden, zu erfassen. Zusätzlich wurde auf der 16. Vertragsstaatenkonferenz der Klimarahmenkonvention in Mexiko die Aufnahme des REDD-Programms beschlossen, das es erlaubt, die Reduktion der Entwaldungs- und Degradierungsraten im Rahmen der nationalen Treibhausgasbilanz zu berücksichtigen (COP16 /Entscheidung 70). Die Umsetzung dieser Programme erfordert die Erfassung der Waldflächen und deren Veränderung auf nationaler Ebene in einem regelmäßigen Turnus mit standardisierten Verfahren (Holmgren et al., 2008). Dies soll durch MRV-Programme (Measurement, Reporting and Verification) umgesetzt werden, die der SBSTA (Subsidiary Body for Scientific and Technological Advice) ausarbeitet (AWG-LCA, 2010). Bereits festgelegt wurde, dass für die Berechnung der Emissionen aus Entwaldung und Degradierung jeweils die aktuellen Richtlinien für die nationalen Treibhausgasinventuren des IPCC verwendet werden sollen (SBSTA, 2009). Diese Richtlinien bestimmen unter anderem, dass standardmäßig die aktuelle Walddefinition der Food and Agriculture Organization of the United Nations (FAO) verwendet werden muss. De facto legt die FAO-Walddefinition also den Standard sowohl für die nationale Treibhausgasinventur als auch für das REDD-Programm fest. Neben dem Klimaschutzabkommen ergeben sich auch Berichtspflichten aus dem Abkommen über den Erhalt der Biologischen Vielfalt. Dies fordert unter anderem, dass die Größe und die Fragmentierung der einzelnen Habitate erfasst und kontrolliert werden soll.

Die Erfassung der Waldfläche und ihrer Verteilung ist für beide Abkommen von zentraler Bedeutung. Zur Erfüllung ihrer Berichtspflichten müssen die Vertragsstaaten transparente und standardisierte Schätzungen der Waldflächen und deren Veränderungen vorlegen. Dafür erarbeiten die jeweiligen wissenschaftlichen Beiräte Standards, die im Rahmen des Waldflächenmonitorings umgesetzt werden müssen. Die Umsetzung erfolgt dann auf nationaler Ebe- 
ne. In Deutschland regelt $\S 41$ a des Bundeswaldgesetzes (BWaldG, Stand 31.Juli 2010), dass alle 10 Jahre eine Bundeswaldinventur durchzuführen ist. Zur Erfüllung der Berichtspflichten aus der Klimarahmenkonvention ist außerdem vorgeschrieben, dass das Bundesministerium für Ernährung, Landwirtschaft und Verbraucherschutz zwischen zwei Bundeswaldinventuren den Kohlenstoffvorrat im Wald schätzen muss. 



\title{
2. Erfassung der Waldfläche: Historische Entwicklung und Stand der Forschung
}

\begin{abstract}
Belege für gezielte terrestrische Aufnahmen zur Erfassung der Waldfläche und der Vorräte reichen bis ins Mittelalter zurück, wie die Einrichtungen des Erfurter Stadtwaldes und des Nürnberger Reichsforstes aus dem 14. Jahrhundert zeigen (Gadow, 2005; Zöhrer, 1980). Diese ersten Waldinventuren waren durch den Mangel an Holz motiviert, der in der Übernutzung und der damit einhergehenden Degradierung der Wälder begründet lag. Holz war zu dieser Zeit der wichtigste Baustoff und Energieträger und deshalb für die Wohlfahrt der Gesellschaft von entscheidender Bedeutung. Zur Verbesserung der Holzversorgung entwickelten sich erste forstliche Lehren zur Bewirtschaftung der Wälder. 1713 erschien als eine der ersten forstwissenschaftlichen Abhandlungen die Schrift von Hans Carl von Carlowitz „Sylvicultura oeconomica, oder haußwirthliche Nachricht und Naturmäßige Anweisung zur wilden Baum-Zucht", in welcher der Verfasser das Prinzip der nachhaltigen Bewirtschaftung der Wälder entwickelte. Carlowitz gilt damit als Begründer der Forstwissenschaften und der Nachhaltigkeit. Um einen Wald nachhaltig zu bewirtschaften, müssen Kenntnisse über die Holzvorräte und Zuwächse des Waldes erworben werden. Dass dazu die Waldfläche erfasst werden muss, weil sie die Grundvoraussetzung ist, auf die sich alle weiteren Kalkulationen beziehen, war schon früh bekannt:
\end{abstract}

\begin{abstract}
„Will man die Größe eines Forstes und den Flächeninhalt der einzelnen Theile oder Distrikte in dem selben wissen, welches absolut nötig ist, um seinen Holzertrag mit Zuverlässigkeit bestimmen zu können; so muss der selbe geometrisch gemessen, gezeichnet und berechnet werden." (Hartig, 1813)
\end{abstract}

Erste und für ihre Zeit sehr präzise methodische Beschreibungen, wie die Waldflächen ermittelt werden sollen, finden sich in Vierenklee \& Meinert (1797) und Hartig (1813). Sie erläutern das Vorgehen bei der Vermessung durch einen Geometer, wobei im Speziellen auf die Grenzziehung eingegangen wird. Die Waldfläche wird anhand der Besitzverhältnisse definiert, so dass der Waldrand entlang der Besitzgrenzen verlief. Noch heute finden sich in vielen deutschen Wäldern alte Grenzsteine als Relikte dieser Waldflächenabgrenzung. Im Anschluss an die Waldfächenerfassung folgte die Forsteinrichtung, welche die Waldfläche in Abteilungen mit homogen bestockten Beständen unterteilt. Die Taxation der Bestände 
erfolgt innerhalb der Abteilungen häufig durch eine Kombination aus Messungen einzelner Variablen und Okularschätzungen. Eine vergleichende Beschreibung, nach welcher Methode diese Gliederung erfolgen sollte, findet sich in Wappes (1893). Das System der Forsteinrichtung ist bis heute in abgeänderter Form das wichtigste Element der operativen Planung der Forstwirtschaft in Deutschland.

Die ersten Waldflächenerhebungen in Deutschland waren meist auf kleinere Ländereien und Waldstücke begrenzt, da sie durch den jeweiligen Besitzer in Auftrag gegeben wurden. Die angefertigten Waldkarten waren oft von hohem ästhetischen Wert, aber ihre Gültigkeit beschränkte sich auf sehr kleine Waldflächen, so dass daraus keine Aussagen über den Waldflächenanteil größerer Gebiete abgeleitet werden konnte. Die Vergleichbarkeit der angefertigten Karten untereinander wurde vor allem durch das Fehlen eines einheitlichen Maßes gemindert (Hartig, 1813).

Durch die Ausbildung des Nationalgedankens, der 1871 durch die Proklamation des ersten Deutschen Kaisers in der Gründung des deutschen Kaiserreiches mündete, wuchs das Interesse an großflächigen Waldinformationen. Eine erste besitzstandsunabhängige Waldflächenerfassung in Deutschland fand 1887 als Forsterhebung für das damalige deutsche Reichsgebiet statt und wurde bis 1937 im zehnjährigen Turnus wiederholt (Schmitz et al., 2008). Diese Forsterhebungen können als erste nationale Waldinventuren gesehen werden, wobei $\mathrm{zu}$ beachten ist, dass es sich nicht um stichprobenbasierte Inventuren, sondern um Zusammenstellungen der Forsteinrichtungsdaten handelt. Die Aussagekraft der Forsterhebungen ist somit eingeschränkt, da die Aufnahmeverfahren in den einzelnen Staaten unterschiedlich waren und keine Abschätzung der Genauigkeit erfolgte.

In dieser Zeit entwickelte sich die Disziplin der Waldinventur sowohl als integraler Teil der Waldbewirtschaftung als auch als Forschungsfeld. Mit Hilfe standardisierter Aufnahmeverfahren sollte eine gesicherte Schätzung der wichtigsten forstlichen Kenngrößen erfolgen. Frühe Erläuterungen zur Anlage von Probeflächen finden sich im "Lehrbuch der Forstwissenschaften für Forstmänner und Waldbesitzer"(Fischbach von, 1886). Es nennt Kriterien für die Anordnung, Größe und Form der Probeflächen. Außerdem wird eine Aufnahme entlang von Linien beschrieben. Die Entwicklung im Bereich der Statistik ermöglichte Anfang des 20. Jahrhunderts erste stichprobenbasierte Waldinventuren. Diese neue Technik wurde zunächst in den skandinavischen Ländern und der USA angewandt, da hier aufgrund der großen Ausdehnung und geringen Bevölkerungsdichte kaum Forsteinrichtungen wie in Deutschland durchgeführt wurden. In Schweden erfolgte die erste nationale Waldinventur 
zwischen 1923-1929 auf Basis von Streifenstichproben (Axelsson et al., 2010). In den USA wurde 1928 durch den Kongress das Forest Inventory and Analysis Programm verabschiedet, das bis 2002 sieben nationale Waldinventuren durchführte (Smith, 2002). In Zentraleuropa etablierten sich die stichprobenbasierten Waldinventuren erst nach Ende des zweiten Weltkrieges: 1960 in der Deutschen Demokratischen Republik, 1987 in der Bundesrepublik Deutschland, 1958 in Frankreich, in den 60er Jahren in Österreich und Spanien und in den 80er Jahren in der Schweiz (Tomppo et al., 2010). Diese späte Entwicklung von stichprobenbasierten nationalen Waldinventuren ist dabei vor allem auf das gut entwickelte System der Forsteinrichtung zurückzuführen.

Die Erfassung der Wälder mittels bildgebender Verfahren hat eine Geschichte, die bis zu den Anfängen der Photographie 1839 zurückreicht. Die systematische Beobachtung der Erdoberfläche wurde jedoch erst durch die Entwicklung geeigneter Flugobjekte ermöglicht. Die Forstwirtschaft nutzte die Fernerkundungstechnik in Form von Luftbildern früh und war maßgeblich an der Entwicklung von Interpretationsverfahren beteiligt. Dabei konzentrierten sich die Anwendungen auf forstwirtschaftliche Fragestellungen, etwa die Erkennung von Waldschäden oder waldwachstumskundliche Auswertung. Für großflächige Waldflächenerfassungen auf regionaler oder nationaler Ebene sind Luftbilder aufgrund ihrer hohen räumlichen Auflösung und kleinen Abdeckung jedoch kaum geeignet.

Die Entwicklung der Raumfahrt und der Satellitentechnik ermöglichen seit den 60er Jahren Erdaufnahmen von großen Gebieten. Die erste globale Landbedeckungskarte wurde aus Daten des Advanced Very High Resolution Radiometer (AVHRR) Systems mit einer räumlichen Auflösung von einem km für die Jahre 1992-93 erstellt (DeFries \& Townshend, 1994; Hansen \& DeFries, 2000). Diese Karten wurden vorwiegend als Eingangsgrößen für globale biogeochemische Modelle verwendet (z. B. Running et al. (1994)). Der dafür vom International Biosphere-Geosphere Programm (IBGP) entwickelte Klassifikationschlüssel enthielt 17 Landbedeckungsklassen, mit fünf unterschiedlichen Waldtypen. (Loveland et al., 2000). Später nutzte Friedl et al. (2002) den selben Klassifikationschlüssel um globale Landbedeckungskarten aus Daten des Moderate Resolution Imaging Spectroradiometer (MODIS) zu erstellen. Bartholomé \& Belward (2005) präsentierten eine globale Landbedeckungskarte basierend auf Daten des VEGETATION-1 Sensors an Bord des SPOT-4 (Satellite Pour l'Observation de la Terre) Satelliten, die eine räumliche Auflösung von einem km über dem Äquator haben. Sie verwendeten das von der FAO entwickelte Klassifikationssystem (engl. Land Cover Classification System, LCCS), das 22 Klassen unterscheidet. Davon sind zehn Klassen durch unterschiedliche Anteile von Kronenüberschirmung gekennzeichnet. Dieser 
als „region-tuned“ bezeichnete Klassifikationsschlüssel unterscheidet sich insofern von dem des IBGP, als dass die Klassen nicht global definiert sind, sondern an bestimmte Regionen der Erde angepasst werden können. Dadurch können die Landbedeckungskarten zwar an die regionalen Besonderheiten angepasst werden, sind aber für einen Vergleich zwischen den Regionen nur eingeschränkt verwendbar. Hansen et al. (2003) entwickelte mit der „continous field of percentage tree cover" Methode einen dritten Ansatz. Diese Methode wurde erfolgreich mit AVHRR- (DeFries et al., 2000) und MODIS- (Hansen et al., 2005) Daten getestet.

Neben den globalen Initiativen gibt es auch auf kontinentaler Ebene verschiedene Projekte zur Erstellung von Landbedeckungskarten. Für die Europäische Union wurden im Rahmen des Programms Coordination of Information on the Environment (CORINE) Karten für die Jahre 1998, 2000 und 2006 mit 44 Klassen und drei thematischen Aufösungen erstellt. Die höchste thematische Auflösung enthält drei Waldtypen (Bossard et al., 2000). Diese Karten stützen sich hauptsächlich auf die visuelle Interpretation von Landsat 7 Thematic Mapper (TM) und Enhanced Thematic Mapper (ETM ${ }^{+}$) Bildern. Pekkarinen et al. (2009) entwickelten eine Waldflächenkarte für die Europäische Union basierend auf den Landsat 7 ETM+ Bildern. Dafür nutzten sie die CORINE Daten als Trainingsdatensatz für das nicht-parametrische Verfahren der $k$-Nächsten Nachbarn $(k-\mathrm{NN})$.

Mittlerweile stehen für fast alle Gebiete der Erde verschiedene Karten zur Verfügung, aus denen Informationen über die Waldfläche gewonnen werden können. Wo diese nicht vorhanden sind, gibt es zumindest genügend Bildmaterial der verschiedenen Satellitensysteme, um solche Karten zu erstellen. Die Verwendungsmöglichkeiten dieser Karten im Rahmen der internationalen Umweltabkommen sind aber aufgrund verschiedener Faktoren eingeschränkt. Ein Problem stellt die Klassendefinition der Landbedeckungsklassen dar. Üblicherweise erfolgt die Kartierung mit Hilfe einer Klassifikation, welche die Pixel eines Bildes anhand ihrer spektralen Signaturen in Landbeckungsklassen überführt. Die spektralen Signaturen werden aus Trainingsgebieten gewonnen, deren Landbedeckungsklasse bekannt ist. Selbst wenn die Trainingsgebiete der jeweiligen Definition genügen, lässt sich kaum nachvollziehen, ob die erstellten Karten einer bestimmten Definition entsprechen. Dies führt sowohl bei den Herstellern als auch bei den Nutzern der Landbedeckungskarten zu Unsicherheiten, da sich die Karten nicht sinnvoll vergleichen lassen und somit im Rahmen von verbindlichen Abkommen kaum zu gebrauchen sind (Herold et al., 2008). 
Ein wesentlicher Grund für die Probleme der Klassendefinition ist in der räumlichen Auflösung der verwendeten Bilder zu sehen. Alle vorgestellten Studien verwenden Bilder mit einer geringen räumlichen Auflösung. Wobei sich "gering“ hier nicht auf einen absoluten Wert, sondern relativ auf das beobachtete Objekt bezieht. Diese von Strahler et al. (1986) vorgeschlagene Terminologie unterscheidet Bilder mit niedriger räumlicher Auflösung, deren Pixel größer als das Objekt sind und Bilder mit hoher räumlicher Auflösung, deren Pixel kleiner als das Objekt sind. Bei einem Wechsel von niedriger zur hoher Auflösung, kommt es somit nicht nur zu einem Anstieg der Auflösung, sondern auch zu einer wesentlichen Änderung des Landschaftskonzeptes, da in hoch aufgelösten Bildern die Objekte direkt beobachtet werden können (Hansen et al., 2002). Erst durch die direkte Beobachtung der Objekte wird es möglich, bestimmte Kriterien, die eine Landbedeckungsklasse definieren, fernerkundlich zu erfassen. Wird zum Beispiel eine Mindestüberschirmung als Kriterium für die Klasse "Wald" festgelegt, kann diese nur dann direkt beobachtet werden, wenn die räumliche Auflösung geringer als die Ausdehnung der Baumkronen ist.

Eine neue Generation von Satelliten stellt schon heute global verfügbare, hoch aufgelöste, multispektrale Bilder zur Verfügung (z. B. GeoEye, IKONOS, QuickBird, RapidEye, SPOT-5, WorldView), die es ermöglichen, Landbedeckungskarten auf regionaler oder sogar nationaler Ebene zu erstellen. Für die nächsten Jahre sind weitere Satellitenmissionen geplant, die zusätzliche hoch aufgelöste Produkte zur Verfügung stellen werden (z. B. SPOT-6 $\& 7$, Pléiades). Ihre hohe Auflösung ermöglicht es bestimmte Kriterien einer Walddefinition (z. B. die Kronenüberschirmung) direkt zu beobachteten. Dies kann die Standardisierung der erstellten Landbedeckungsklassen verbessern.

Es ist abzusehen, dass Fernerkundungsdaten für die Erfassung der Waldflächen im Rahmen internationaler Umweltabkommen eine zentrale Rolle spielen werden (DeFries et al., 2006; Holmgren et al., 2008). Durch den Einsatz von Fernerkundungsdaten können flächendeckende Informationen über die Waldfläche in relativ kurzer Zeit für große Flächen bereit gestellt werden. Dies ist besonders in den tropischen Ländern relevant, wo aufgrund der vielfältigen Besitzverhältnisse und dem geringen Erschließungsgrad der Wälder, terrestrische Waldinventuren sehr aufwendig sind. Außerdem stellen historische Satellitenaufnahmen, wie sie zum Beispiel seit 1972 durch die verschiedenen Sensoren des Landsat Systems aufgenommen wurden, häufig die einzige Information über die Ausdehnung der Waldfläche zu früheren Zeitpunkten dar. Die Erfüllung der Berichtspflichten aus den Umweltabkommen (siehe Abschnitt 1.4) benötigt standardisierte Methoden zur Waldflächenerfassung, die eine unverzerrte und effektive Schätzung der Waldfläche mit Hilfe von Fernerkundungsdaten 
ermöglichen und den Anforderungen der Abkommen genügen. Dafür müssen die Faktoren, welche die Qualität der erstellten Waldflächenkarten maßgeblich beeinflussen, bekannt sein.

Im nächsten Kapitel werden vier solcher im folgenden als „kritisch“ bezeichnete Faktoren vorgestellt. 


\section{Kritische Faktoren der fernerkundlichen Waldflächenerfassung}

\subsection{Walddefinition}

Soll die Waldfläche erfasst werden, muss zunächst eine klare Abgrenzung des Waldes erfolgen. Üblicherweise wird dazu eine Wald- und (damit verbunden) eine Waldranddefinition verwendet. Bevor auf die verschiedenen Möglichkeiten einer naturwissenschaftlichen Walddefinition eingegangen wird, soll eine kurze etymologische Betrachtung des Waldbegriffes erfolgen.

Was ist ein Wald? Obwohl diese Frage schon vielfach diskutiert worden ist, kann sie nicht als beantwortet gelten. Denn die Definition des Waldes ist eng verbunden mit den soziokulturell geprägten Vorstellungen einer Gesellschaft und der Art der Bedeutung, die sie dem Wald beimisst. Laut Maurer \& Rupp (1974) kann das Wort Wald auf das Wort Wituw zurückgeführt werden. Hasel \& Schwartz (2006) weisen daraufhin, dass ab dem Mittelalter die Begriffe Wald (silva) und Forst (forestris) unterschieden worden, wobei der forestris, der in königlichem Besitz befindliche, eingeforstete und silva der nicht eingeforstete Teil des Waldes ist. Der Forst erstreckt sich dabei nicht nur auf den mit Bäumen bestockten Teil, sondern schließt ebenso in den Waldgebieten befindliche Gewässer, Äcker, Weinberge, Weiden und Dörfer ein. Diese Trennung der Begriffe geht einher mit der zunehmenden Nutzung der Wälder. Für eine etymologische Erläuterung zum englischen Wort forest, welches sowohl den Wald als auch den Forst beschreibt, sei auf Putz \& Redford (2010) verwiesen. Diese kurze Betrachtung der Entwicklung des Wortes Waldes zeigt drei Aspekte: i) Der Waldbegriff umfasst sowohl eine Beschreibung der Landbedeckung als auch der Landnutzung, ii) die Verständigung darüber, was einen Wald ausmacht, ist eng mit den gesellschaftlichen Ansprüchen an den Wald verbunden und iii) dieses Verständnis ändert sich fortlaufend, so wie sich auch die Form der Nutzung ändert

Die Frage, was ein Wald sei, muss also ständig neu beantwortet werden. Dies spiegelt sich in der Vielzahl von Walddefinitionen wieder, die rechtliche, ökologische und soziale Aspekte des Waldbegriffes berücksichtigen. Die vermutlich umfangreichste und aktuellste 
Sammlung der verschiedenen Walddefinitionen findet sich bei Lund (2011). In einer dazu gehörigen Publikation ordnet Lund (2002) die Definitionen in drei Gruppen: Landnutzung, Landbedeckung und Andere (z. B. administrative Einheiten).

Wenn die Konzepte, Ansprüche und Nutzung des Waldes weltweit so unterschiedlich sind, wie kann dann eine Vereinheitlichung erfolgen? Zwei Strategien sind denkbar. Zum einen könnten einheitliche Walddefinitionen festgelegt und für die beteiligten Parteien vorgeschrieben werden. Zum anderen könnten Definitionsrahmen gegeben werden, die eine gewisse Flexibilität ermöglichen und trotzdem durch Transformation auf einen einheitlichen Standard gebracht werden können.

Der naheliegendste Ansatz ist, zunächst die verschiedenen Walddefinitionen miteinander zu vergleichen um den kleinsten gemeinsamen Nenner zu ermitteln. Lund (2002) hat dies getan und kam zu dem Ergebnis, dass Wald im weitesten Sinne eine mit Bäumen bestandene Fläche ist. Dieser kleinste gemeinsame Nenner ist jedoch wenig geeignet, um über Wald zu kommunizieren. Daher sind weitere Kriterien notwendig, die in quantitative und qualitative Kriterien unterschieden werden können (Kleinn, 1991, 1992, 2001; Lund, 2002; Vidal et al., 2008). Qualitative Kriterien stützen sich meistens auf andere Begriffe, die ausreichend klar zu sein scheinen. Häufig werden sie verwendet, um Ausschlusskriterien zu formulieren, etwa ob Obstplantagen oder Weihnachtsbaumkulturen zur Waldfläche gehören oder ob Bambuspflanzen als Bäume anerkannt werden. Oft führen diese Kriterien jedoch zu Entscheidungsunsicherheiten, da nicht geregelt wird, was eine Obstplantage, eine Weihnachtsbaumkultur, etc. ist. Insofern führen sie in vielen Fällen zu einer Verlagerung der Definitionslücken. Im Gegensatz dazu geben quantitative Kriterien klare, eindeutig messbare Größen vor, die erreicht werden müssen, damit eine bestimmte Fläche als Wald anerkannt wird. Nach Lund (2002) sind die weltweit am häufigsten verwendeten quantitativen Kriterien:

1. Flächengröße

2. Kronenüberschirmung

3. Baumhöhe oder Bestandeshöhe

4. Breite der Waldfläche

In einer europäischen Studie zur Harmonisierung der nationalen Waldinventuren wurden verschiedene Walddefinitionen untersucht. Auch hier zeigte sich, dass die Kriterien Flächengröße, Kronenüberschirmung und Breite der Waldfläche die am häufigsten verwendeten sind (Vidal et al., 2008). Die gewählten Schwellenwerte für diese Kriterien sind jedoch sehr 
unterschiedlich.

Verschiedene Arbeiten widmen sich den Effekten der Schwellenwerte auf die Waldflächenschätzung. Traub et al. (2000) untersuchten für einige europäische Länder, auf Basis von Stichproben, Schwellenwerteffekte für die nationalen Waldflächenschätzungen anhand simulierter Kronenkarten. Sie zeigten, dass sich die Waldflächen um maximal 25\% ändern, wenn die Schwellenwerte variieren. Mathys et al. (2006) untersuchten die Auswirkung verschiedener Wald- und Waldranddefinitionen auf die Waldflächenschätzung in der Schweiz. Sie konnten darlegen, dass der Einfluss der Kronenüberschirmung am größten ist. Sasaki \& Putz (2009) diskutierten die verschiedenen Schwellenwerte vor dem Hintergrund der Walddegradierung und schlussfolgerten, dass ein Schwellenwert von $40 \%$ Kronenüberschirmung besser geeignet sei, um die Degradierung von tropischen Wäldern zu detektieren. Verchot et al. (2007) analysierten die Änderung des Schwellenwertes der Kronenüberschirmung für vier Untersuchungsgebiete in Kenia, Uganda, Bolivien und Ecuador anhand von MODIS-Daten (Hansen et al., 2003). Zomer et al. (2008) erweiterten diese Forschungen auf alle Länder, die nicht im Annex 1 des Kyotoprotokolls aufgeführt sind, um herauszufinden, wie sich der Schwellenwert für die Kronenüberschirmung auf die Waldfläche und, damit verbunden, die für das Clean Development Mechanism (CDM) Schema nutzbare Landesfläche auswirkt.

Eine der wenigen Studien, die sich explizit mit dem Einfluss der Mindestbreite befasst, ist die von Traub et al. (2000). Sie demonstrierten anhand von simulierten Karten, dass die Mindestbreite einen großen Einfluss auf die Waldflächen hat. Im Gegensatz dazu zeigten die Analysen von Mathys et al. (2006) nur einen moderaten Einfluss der Breite.

Es kann festgestellt werden, dass die Mindestüberschirmung das am häufigsten verwendete Kriterium aktueller Walddefinitionen ist und einen starken Einfluss auf die Waldflächenschätzung hat (Mathys et al., 2006; Verchot et al., 2007; Zomer et al., 2008). Außerdem ist es ein quantitatives Kriterium, das sowohl in terrestrischen als auch durch fernerkundliche Aufnahmen erfasst werden kann. Wird es in Kombination mit anderen quantitativen Kriterien (z. B. der Mindestgröße oder -breite einer Waldfäche) angewendet, stellt es die Grundlage dar, auf der die anderen Kriterien aufbauen. Beispielsweise muss erst die Waldfläche auf Basis der Mindestüberschirmung bestimmt werden, bevor geprüft werden kann, ob die geforderte Mindestgröße erreicht ist. Aufgrund dessen wird in der vorliegenden Arbeit das Kriterium Mindestüberschirmung als kritischer Faktor stellvertretend für die Walddefinition untersucht. 


\subsection{Waldranddefinition}

„Jede Messung sezt aber bestimmte Grenzen voraus. Es müssen daher die äußeren Grenzen der zusammenhängenden Waldung und der abgesondert liegenden Distrikte, so wie auch die Grenzen in dem Walde gelegener Äcker und Wiesen in Richtigkeit gebracht werden, ehe man die Messung selbst vornehmen kann" (Hartig, 1813).

Wie Hartig beschreibt, ist neben den Kriterien der Walddefinition die exakte Festlegung des Waldrandes von entscheidender Bedeutung für die Ausweisung von Waldflächen. Der Waldrand markiert die Grenzlinie zwischen Wald und Nicht-Waldflächen. Eine explizite Definition des Waldrandes findet sich in der Literatur kaum und erfolgt nur in wenigen Waldinventuren. Dabei ist die eindeutige Identifikation der Lage des Waldrandes, sowohl im Rahmen von terrestrischen Inventuren als auch bei der fernerkundlichen Waldflächenerfassung, ein real bestehendes Problem. Eine der wenigen Ausnahmen ist die Walddefinition der Schweizer Nationalinventur, die den Waldrand wie folgt definiert:

„Die Waldbegrenzungslinie (WBL) ist die längste mögliche Verbindungslinie aller Waldrand bildenden und dem Wald vorgelagerten Bestockungsglieder (gemeint sind Waldbäume z. B. Fichten, Weiden oder Birken, aber nicht Obstbäume), die auf Brusthöhe (BHDMesshöhe) von Stammitte zu Stammitte gemessen horizontal höchstens 25m voneinander entfernt sind." (Keller, 2005)

Die WBL wird unter Berücksichtigung eines maximalen Baumabstandes festgelegt, weshalb dieses Vorgehen im folgenden als Abstandsverfahren bezeichnet wird. Erst nach der Festlegung der WBL wird geprüft ob andere Kriterien (Überschirmumg, Breite, Höhe) innerhalb der eingeschlossenen Fläche erfüllt werden. Zu erwähnen ist, dass für die Schweizer Nationalinventur die Mindestüberschirmung als Funktion der Breite einer Waldfläche vorgegeben wird. Das heißt, dass schmale Waldflächen auch mit geringen Überschirmungen noch als Wald gelten, wohingegen Waldfächen, welche breiter als $25 \mathrm{~m}$ sind, zu $100 \%$ überschirmt sein müssen. Dieses Verfahren wurde bisher in drei Schweizer Nationalinventuren verwendet, wobei die Methode sowohl zur Interpretation von Luftbildern als auch bei den terrestrischen Aufnahmen angewendet wurde. Eine detaillierte Beschreibung der Schweizer Nationalinventur findet sich in Brändli et al. (2001).

Eine andere Möglichkeit zur Definition des Waldrandes ist die Anwendung einer fixen Referenzfläche. Im Unterschied zum Abstandsverfahren wird in dieser, hier als Referenzflächen- 
verfahren bezeichneten, Methode zunächst eine Referenzfläche mit fixer Größe und Form unabhängig vom Baumbewuchs festgelegt. Innerhalb dieser Referenzfläche wird die Kronenüberschirmung aus dem Verhältnis des überschirmten und des nicht überschirmten Teils der Referenzfläche bestimmt. Dadurch wird der Waldrand maßgeblich durch die Größe der Referenzfläche bestimmt, denn die Waldgrenze verläuft entlang des Überschirmungsgradienten, genau entlang der Linie an welcher die Mindestüberschirmung unterschritten ist. Diese Methode wurde erstmals von Kleinn (1991) beschrieben, welcher untersuchte wie sich die Größe der fixen Referenzfläche zur Bestimmung der Kronenüberschirmung auf die geschätzte Waldfläche auswirkt. In dieser und in folgenden Studien (Kleinn, 1992, 2001; Magdon \& Kleinn, 2012) konnte gezeigt werden, dass die Definition einer Referenzfläche für die Waldfläche von entscheidender Bedeutung ist, indem sie die Lage des Waldrandes variiert. Damit unterscheidet sich dieses Verfahren vom Abstandsverfahren im Hinblick auf ihre Waldranddefinition deutlich.

In der vorliegenden Arbeit wird das Referenzflächenverfahren zur Definition des Waldrandes verwendet, da es sich im Rahmen von fernerkundungsgestützten Waldkartierungen für eine automatisierte Delinierung der Waldflächen eignet. Außerdem weist Lanz (2005) auf Probleme des Abstandsverfahrens hin, da die Lage der WBL allein durch die Definition eines Mindestabstandes nicht eindeutig festgelegt ist.

\subsection{Landschaftsmodell}

Üblicherweise werden LSM auf Basis des PCM-Modells berechnet. Als Grundlage dienen planimetrische Karten, welche die Landschaft als horizontale Projektion in einem kartesischen Raum darstellen. Topographische Informationen sind in diesem Landschaftsmodell nicht berücksichtigt. Die Topographie spielt für viele ökologische Prozesse eine entscheidende Rolle. Hydrologische Prozesse und Strahlungsbilanzen werden direkt davon beeinflusst und haben weitreichende Auswirkungen auf die Ökosysteme. Das Verhalten der Arten und damit verbunden ihre Populationsdynamik hängen von den verfügbaren Ressourcen ab. Bei einem räumlich begrenzten Angebot dieser Ressourcen werden die Vorgänge daher mit einem dreidimensionalen Modell wesentlich besser erfasst als in planimetrischen Modellen (Jenness, 2004). Die Flächen und Distanzen, wie sie in einer Karte abgebildet sind, entsprechen demnach nicht der Landschaftsperzeption der Arten. Für die sinnvolle Verknüpfung der Landschaftsmodelle mit dem Verhalten der Arten ist dies aber eine Grundvoraussetzung. 
Bisher wurde die Topographie bei der Berechnung von LSM in der Landschaftsökologie kaum berücksichtigt. Eine Ausnahme bildet die Arbeit von Dorner et al. (2002). Sie beschrieben drei Ansätze zur besseren Integration von topographischen Informationen: i) die Anpassung der klassischen Indices durch Berücksichtigung der wahren Oberfläche ii) die Entwicklung von neuen Indices, welche die topographischen Gegebenheiten auf Patchebene erfassen und iii) die statistische Modellierung. Auch Hoechstetter et al. (2008) und Walz et al. (2007) untersuchten explizit die Effekte der Topographie auf die Berechnung der LSM. Sie konnten zeigen, dass in Abhängigkeit der Komplexität der Topographie signifikante Unterschiede für die Flächen, den Umfang und die mittlere Entfernung der Patches ermittelt werden. Des Weiteren untersuchten sie drei Form-Metriken, welche keinen einheitlichen Trend zeigten.

In der vorliegenden Arbeit wird als dritter kritischer Faktor das Landschaftsmodell untersucht, da es sowohl auf die Berechnung der Waldfläche als auch auf die Berechnung der LSM einen Einfluss haben kann. Für die Untersuchung werden zwei- und dreidimensionale Modelle verglichen.

\subsection{Beobachtungsmaßstab}

Jede Beobachtung erfolgt mit einem bestimmten Maßstab, der räumlicher oder auch zeitlicher Art sein kann. Räumliche Muster entstehen durch Prozesse, die auf bestimmten Skalen stattfinden, auf anderen jedoch nicht beobachtet werden können (Gotway \& Young, 2002). Die Wahl des richtigen Maßstabes ist daher für die Beobachtungen dieser Prozesse von großer Bedeutung, da eine Veränderung des Betrachtungsmaßstabes zu unterschiedlichen Schlussfolgerungen bezüglich der landschaftsprägenden Prozesse führen kann. Ein solcher Effekt wird als Skaleneffekt bezeichnet. Diese wurden in vielen Bereichen der Natur- und Sozialwissenschaften beschrieben und intensiv diskutiert. Sie spielen besonders bei der Erfassung und Modellierung räumlicher Prozesse eine wichtige Rolle. Marceau (1999) gibt einen Überblick über die verschiedenen Themenfelder und führt in die Terminologie ein.

Skøien \& Blöschl (2006) beschreiben die drei Elemente des Maßstabes: Auflösung, Ausdehnung und Bezugsfläche als Skalentriplett. Übertragen auf die kartographische Darstellung von Waldflächen kann dies wie folgt interpretiert werden: Die Auflösung legt die Größe des kleinsten Objektes, das erkannt werden kann, fest. In Fernerkundungsdaten wird dies durch räumliche Auflösung der Pixel, also den Teil der Erdoberfläche über welche die elektro- 
magnetische Strahlung integriert wird, beschrieben. In Vektordaten wird meist der Begriff Minimum Mapping Unit (MMU) verwendet. Er beschreibt das kleinste Polygon, das erfasst wird. Die Ausdehnung beschreibt das Gebiet, welches abgedeckt wird, also das größte Objekt, das vollständig erfasst werden kann. Die Bezugsfläche legt die Referenzfläche fest, welche berücksichtigt wird, um einen Punkt einer bestimmten Klasse zuzuordnen.

Umfangreiche Untersuchungen beschäftigen sich mit einzelnen oder mehreren Elementen des Skalentripletts in verschiedenen Fachbereichen. Unter anderem für die Fernerkundung im Allgemeinen (Atkinson \& Curran, 1995; Mareceau \& Geoffrey, 1999; Strahler et al., 1986; Woodcock \& Strahler, 1987), für landschaftsökologische Fragestellungen (Benson \& MacKenzie, 1995; Jelinski \& Wu, 1996; O'Neill et al., 1996; Saura \& Castro, 2007; Turner et al., 1989; Wu, 2004) und im Rahmen der Waldflächenerfassung (Frohn \& Hao, 2006; Nelson et al., 2009; Traub \& Kleinn, 1999; Zheng et al., 2009). Die Ergebnisse dieser vielfältigen Studien zeigen klare, zum Teil sehr unterschiedliche Effekte der einzelnen Skalenelemente bei der Erfassung und Beschreibung von Landschaften.

Im Rahmen von Landschaftsanalysen treten Skaleneffekte auf, wenn Landkarten unterschiedlichen Maßstabes verglichen werden. Solche Situationen ergeben sich zum Beispiel bei der Erstellung von Zeitreihen mit Fernerkundungsdaten, anhand derer Änderungen der Landschaft beschrieben werden. Skaleneffekte führen hier zu inhärenten Variationen, welche zu verzerrten Schlussfolgerungen über die Änderungen der Landschaft führen können. Eine Möglichkeit, diesem Problem zu begegnen, ist die Modellierung der Skaleneffekte. Wu (2004) untersuchte die Wirkung der beiden Elemente Auflösung und Ausdehnung auf 17 verschiedene LSM und beschrieb drei potentielle Skalierungsfunktionen. Einige Effekte konnten mit kontinuierlichen Funktionen (z. B. exponentiellen, linearen oder logarithmischen) beschrieben werden. Andere zeigten sprunghafte Reaktionen und wieder andere ließen keine Modellierung zu. Tendenziell konnten Änderungen in der Auflösung besser modelliert werden als Änderungen der Ausdehnung. Frohn \& Hao (2006) untersuchten 16 LSM hinsichtlich ihrer Skalierbarkeit. Auch sie konnten zeigen, dass sich einige der untersuchten Metriken sehr gut durch Skalierungsfunktionen beschreiben lassen und andere nicht. Saura \& Castro (2007) studierten die Möglichkeiten Skalierungsfunktionen für eine Subpixel-Klassifikation einzusetzen. Dabei verglichen sie acht LSM, die zum einen mit den Skalierungsfunktionen und zum anderen durch Aggregation der Landbedeckungskarten berechnet wurden. Dieser Multiskalenansatz bot neben der Subpixel-Klassifikation zugleich auch die Möglichkeit die Skalierungsfunktionen zu evaluieren. Sie schlussfolgerten, dass nur einige der von Wu (2004) beschriebenen Skalierungsfunktionen skalenunabhängige Berechnungen zulassen. Gustafson 
\& Parker (1992) untersuchten das Verhalten verschiedener LSM als Funktion des Anteils einer Klasse an der Landschaft. Dabei wurde gezeigt, dass die untersuchten LSM zum Teil sensitiv auf den Anteil reagieren. Zusammenfassend lässt sich feststellen, dass alle drei Elemente des Skalentripletts große Wirkung auf die Beschreibung von räumlichen und zeitlichen Prozessen haben. In den vielfältigen Studien wird deutlich, dass diese Wirkung sehr unterschiedlich sein kann und dass eine Modellierung der Effekte nur in einigen Fällen möglich ist.

In den erwähnten Arbeiten werden die Effekte der räumlichen Auflösung auf die LSM beschrieben, indem Landbedeckungskarten aggregiert werden. Die Veränderung der Auflösung wirkt also direkt auf die Karte. Wenn Waldflächen auf Basis von Mindestüberschirmung und Referenzfläche erfasst werden, wirkt die Änderung der räumlichen Auflösung jedoch nicht direkt auf die Waldfläche, sondern indirekt über die Referenzfläche. Folgendes Beispiel verdeutlicht, dass die Wirkung der Auflösung von der Größe der Referenzfläche und der Mindestüberschirmung abhängt:

Für eine quadratische Referenzfläche von $500 \mathrm{~m}^{2}$ soll eine Wald-/Nicht-Waldentscheidung getroffen werden. Diese Fläche wird durch Fernerkundungsdaten mit einer räumlichen Auflösung von 5m Kantenlänge pro Pixel abgedeckt, so dass 20 Pixel in die Referenzfläche fallen. Anschließend werden die von Kronen überschirmten Pixel gezählt und ihr Anteil bestimmt. Bei 10 Pixeln sind die Werte $p=0.5,0.1,0.15, \ldots 1$ möglich. Ist der Wert größer als die geforderte Mindestüberschirmung wird der Punkt als Wald klassifiziert. Wenn eine Überschirmung von $\geq 10 \%$ gefordert ist, wird der Punkt als Wald klassifiziert, wenn mindestens 2 Pixel in der Referenzfläche von Kronen überschirmt sind. Wird die räumliche Auflösung von $5 \mathrm{~m}$ auf $10 \mathrm{~m}$ Kantenlänge verdoppelt, sind bei gleicher Referenzfläche noch fünf Pixel zu prüfen, was die Überschirmungswerte $p=0.2,0.4, \ldots 1$ zulässt. Ein Pixel würde in diesem Fall ausreichen um der geforderte Mindestüberschirmung zu genügen. Das bedeutet, dass bei $5 \mathrm{~m}$ Auflösung mindestens $2 \times 5^{2}=50 \mathrm{~m}^{2}$ und bei einer Auflösung von $10 \mathrm{~m} 1 \times 10^{2}=100 \mathrm{~m}^{2}$ von Kronen überschirmt sein müssen, damit die selbe Referenzfläche als Wald klassifiziert wird. 
Bei der Verwendung von dreidimensionalen Landschaftsmodellen muss neben der räumlichen Auflösung, welche die horizontale Ausdehnung eines Pixels beschreibt, auch die vertikale Aufösung der Pixel berücksichtigt werden. Sie gibt den Wertebereich an, mit dem die Höheninformationen gespeichert sind und kann mit der radiometrischen Auflösung eines Sensors verglichen werden. Die vertikale Auflösung wird zum einen durch die Messverfahren, mit dem die Höheninformationen erfasst werden, und zum anderen durch das Datenformat, in dem diese Informationen gespeichert werden, bestimmt.

In der vorliegenden Arbeit sollen zwei kritische Skaleneffekte untersucht werden: i) die horizontale Auflösung der Landschaften, um zu untersuchen welche Effekte sich für das Referenzflächenverfahren ergeben und ii) die vertikale Auflösungen der Geländemodelle bei der Berechnung von LSM auf Basis von dreidimensionalen Landschaftsmodellen. 



\section{Zielsetzung und Gliederung der Arbeit}

Die fernerkundliche Erfassung der Waldfläche und deren Fragmentierung im Rahmen von internationalen Umweltabkommen sind Gegenstand dieser Arbeit. Zielsetzung ist es, methodische und technische Probleme zu identifizieren und ihre Relevanz innerhalb der Monitoringsysteme zu analysieren. Im vorangestellten ersten Teil der Arbeit wurden vier kritische Faktoren, die einen Einfluss auf die Erfassung der Waldfläche haben können, anhand der Literatur identifiziert. Eine umfassende Analyse auf der Grundlage von Simulationen mit künstlichen Landschaften erfolgt im zweiten Teil der Arbeit. Dabei sollen folgende Forschungsfragen bearbeitet werden:

1. Wie wirken die vier kritischen Faktoren: Walddefinition, Waldranddefinition, Beobachtungsskala und Dimension des Landschaftsmodells auf die Schätzung der Waldfläche und die Landschaftsstrukturmaße?

2. Gibt es Wechselwirkungen zwischen den kritischen Faktoren?

3. Welchen Einfluss hat die Komposition und Konfiguration der beobachteten Landschaft auf die Wirkung der kritischen Faktoren?

Ausgehend von der Analyse wird im dritten Teil der Arbeit ein Konzept zur fernerkundlichen Waldflächenerfassung entwickelt, das die Wirkung der kritischen Faktoren berücksichtigt und somit zur Standardisierung der fernerkundlichen Waldflächenerfassung beitragen kann. Dabei wird angestrebt ein Verfahren zu entwickeln, welches geeignet ist, Waldflächenkarten und Fragmentierungsmetriken für die Berichterstattung im Rahmen des UNFCCC, REDD und UNCBD zu erstellen. Folgende Forschungsfragen werden bearbeitet:

1. Wie können Kriterien der FAO-Walddefinition in einen Klassifikationsschlüssel zur Auswertung von Fernerkundungsdaten übertragen werden?

2. Welche Bildverarbeitungschritte sind notwendig, um einen solchen Klassifikationsschlüssel für eine Bildklassifikation zu verwenden?

Im vierten Teil der Arbeit werden mit dem entwickelten Verfahren Waldflächenkarten für zwei Untersuchungsgebiete in Costa Rica, auf Basis von RapidEye-Satellitenbildern, erstellt. Dabei soll getestet werden, ob das entwickelte Verfahren in der praktischen Anwendung funktioniert. 
Folgende Forschungsfragen ergeben sich für den vierten Teil der Arbeit:

1. Ist das entwickelte Verfahren geeignet, standardisierte Waldflächenkarten für zwei sehr unterschiedliche tropische Landschaften zu erstellen?

2. Sind die Bilder des RapidEye-Satellitensystems geeignet, um standardisierte Waldflächenkarten zu erstellen?

An Ende der Arbeit erfolgt eine Schlußbetrachtung, die neben einer Zusammenfassung der wesentlichen Ergebnisse auch Ansätze für weitergehende Forschung aufzeigt.

Im Rahmen dieser Dissertation sind bislang zwei Veröffentlichungen entstanden. In Magdon \& Kleinn (2012) wurden Teile der Simulationen und Auswertung, die im Teil I und II der vorliegenden Arbeit beschrieben worden sind, veröffentlicht. Die Teile III und IV sollen in Magdon et al. (2013) veröffentlicht werden. Im Interesse der Lesbarkeit wurde darauf verzichtet, permanent auf die beiden eigenen Veröffentlichungen zu verweisen. Bei wörtlichen Zitaten und Übernahme von Abbildungen aus den Publikationen erfolgt der entsprechende Quellennachweis. 


\section{Teil II.}

Sensitivitätsanalyse der kritischen Faktoren 



\section{Einleitung Simulation}

\subsection{Ausgangssituation und Zielsetzung der Simulation}

In Kapitel 3 wurden auf Basis einer Literaturrecherche vier Faktoren identifiziert, die bei der Erfassung der Waldfläche einen kritischen Einfluss haben können. Um operationale Verfahren zur Waldflächenerfassung entwickeln zu können, müssen sowohl die Wirkungsmechanismen der einzelnen Faktoren als auch deren Wechselwirkungen untereinander und mit der zu beschreibenden Landschaft verstanden werden. Zielsetzung ist es, auf Basis von Simulationen die kritischen Faktoren der fernerkundlichen Waldflächenerfassung und deren Wechselwirkungen zu beschreiben. Anschließend soll eine Bewertung hinsichtlich der Bedeutung der Faktoren im Rahmen des Waldflächenmonitoring erfolgen. Dabei müssen zwei Gruppen von Faktoren berücksichtigt werden:

1. Faktoren, die durch die Gestaltung des Aufnahmeverfahrens gesteuert werden können, im Folgenden als endogene Faktoren bezeichnet.

2. Faktoren, die sich aus der zu beobachtenden Landschaft ergeben, im Folgenden als exogene Faktoren bezeichnet.

Es ist anzunehmen, dass zwischen beiden Gruppen Wechselwirkungen auftreten. Zum Beispiel kann erwartet werden, dass die beobachtete Kronenüberschirmung in einem Landschaftsausschnitt maßgeblich von der räumlichen Auflösung der Karte abhängt oder die Distanz zwischen zwei Punkten vom Relief der Landschaft. Um Wechselwirkungen zwischen endogenen und exogenen Faktoren zu analysieren, müssen beide gezielt variiert werden können. Da eine systematische Variation der exogenen Faktoren in realen Landschaften nicht möglich ist, bietet sich die Simulation von räumlichen Landschaften, basierend auf Landschaftsmodellen, an. Für die Simulation werden binäre Landschaften mit zwei Klassen (Baumkrone/Nichtbaumkrone) verwendet, in welchen die drei exogenen Faktoren i) Gesamtanteil an Baumkronen ii) räumliche Verteilung der Baumkronen und iii) Topographie der Landschaft systematisch variiert werden. 


\subsection{Zielgrößen des Waldflächenmonitorings}

Primäre Zielgröße des Waldflächenmonitorings ist die Größe der Waldfläche. Daneben soll aber auch die räumliche Struktur der Waldflächen auf Basis der LSM erfasst werden, um die Fragmentierung zu beschreiben. Bei der Anwendung dieser Metriken muss zwischen drei Skalenebenen differenziert werden: Landschafts-, Klassen- und Patchebene. Gegenstand dieser Arbeit ist die Waldflächenerfassung, daher werden nur zwei Klassen, Wald und Nichtwald, berücksichtigt. So ist die Klassenebene äquivalent zur Landschaftsebene. Zum Beispiel entspricht die Randlänge aller Waldpatches der Gesamtumfanglänge aller Patches der Landschaft, da Wald die einzige Klasse ist. Trotz dieser Reduktion ist die Vielzahl der Metriken immer noch unübersichtlich, was eine Auswahl erfordert.

Grundsätzlich sollten die LSM geeignet sein, die Muster der Landschaft zu beschreiben, welche für die gegebene Fragestellung relevant sind. Die vorliegende Arbeit diskutiert die Verwendung der LSM für die Erfassung der Waldfläche und als potentielle Indikatoren im Rahmen eines Biodiversitätsmonitorings. Für ersteres ist die gesamte Waldfläche in einem Gebiet die Zielgröße. Für die Verwendung als Biodiversitätsindikator muss ein Zusammenhang zwischen der Landschaftsstruktur und den Prozessen, welche die Artenzahl in einem Waldgebiet maßgeblich bestimmen, hergestellt werden. In Abschnitt 1.3.3 wurde ein solcher Zusammenhang zwischen den populationsdynamischen Prozessen und der Struktur einer Waldlandschaft dargestellt und wichtige Landschaftscharakteristiken identifiziert. Im folgenden Absatz werden diesen verschiedene LSM zugeordnet und in Tabelle 1 zusammengefasst. Bei der Auswahl der Metriken wurden ausschließlich häufig verwendete gewählt, da eine Untersuchung ihrer Sensitivität besonders relevant erscheint. Weitere Kriterien für die Auswahl der Metriken waren die Möglichkeit zur Berechnung im dreidimensionalen Raum und die Einfachheit der Interpretation der Metriken.

Waldanteil (COVER) Der Waldanteil ist der Anteil der Landschaft, der von Wald bedeckt ist. Er ist ein Maß der Landschaftskomposition. Der Waldanteil ist die zentrale Größe des Waldflächenmonitorings. Bei der Berechnung von Kohlenstoffbilanzen ist der Waldanteil, neben der Kohlenstoffdichte, die grundlegende Variable. Als Biodiversitätsindikator kann er genutzt werden, um die Gesamtgröße der zur Verfügung stehenden potentiellen Habitate zu schätzen. Es kann davon ausgegangen werden, dass ein Rückgang des Waldanteils in einer Landschaft langfristig auch zu einem Rückgang der auf den Wald als Habitat angewiesenen Arten führt. Der Waldanteil lässt keine Schlussfolgerungen bezüglich der räumlichen Ver- 
teilung der Waldflächen in der Landschaft (Landschaftskonfiguration) zu, daher kann die Beurteilung der Habitatqualität nicht ausschließlich auf Basis des Waldanteils erfolgen.

Patchgröße(SIZE) Die Patchgröße beschreibt die Ausdehnung der Waldflächen und wird auf der Patchebene bestimmt. Auf Landschaftsebene kann sie auch als mittlere Größe aller Patches angegeben werden. In diesem Fall ist es oft hilfreich neben dem Mittelwert auch ein Streuungsmaß anzugeben, um die Variabilität der Patches zu beschreiben.

Patchanzahl (N_PATCH) Die Anzahl der Patches in einem Gebiet ist ein Maß der Landschaftskonfiguration, das auf der Landschaftsebene berechnet werden kann. Sie wird entweder absolut oder als Dichte angegeben. Die Verwendung als Dichtemaß ist immer dann sinnvoll, wenn Gebiete unterschiedlicher Größe miteinander verglichen werden. Die Patchanzahl bestimmt die Menge der potentiellen Habitate und kann somit als Biodiversitätsindikator genutzt werden.

Patchform(PARA,SHAPE,FRAC) Die Form eines Patches beschreibt seine geometrische Komplexität, die einen Einfluss auf seine Eignung als Habitat haben kann. Beispielsweise beschrieb Forman (1995a) drei form-function-principles welche den Einfluss der Patchform auf Pflanzen und Tiere darstellen. Auch Moser et al. (2002) zeigten starke Korrelationen zwischen der Patchform und der Artenzahl krautiger Pflanzen und Moose für landwirtschaftlich geprägte Flächen in Österreich.

Die einfachste Beschreibung der Patchform ist das Verhältnis zwischen der Fläche $a$ und dem Umfang $p$ eines Patches $(P A R A)$. Dabei führen unregelmäßige Patches zu einem großen Umfang bei geringer Fläche. Das einfache Verhältnis Umfang/Fläche hängt von der Größe der Patches ab (größere Flächen führen zu einem kleineren Verhältnis)(Leitão et al., 2006).

$$
P A R A=\frac{p}{a}
$$

Um das Problem der Abhängigkeit von der Patchgröße zu überwinden, wurden weitere Metriken entwickelt, um die Form zu beschreiben. SHAPE ist ein sehr häufig verwendetes LSM, welches das Verhältnis zwischen dem Umfang eines Patches und dem Umfang eines 
flächengleichen Kreises bildet. Dieses Verhältnis ist unabhängig von der Größe des Patches und kann daher genutzt werden, um Landschaften mit sehr unterschiedlichen Patchgrößen zu vergleichen.

$$
S H A P E=\frac{0.25 * p}{\sqrt{a}}
$$

Ein weiteres häufig verwendetes LSM zur Beschreibung der Komplexität von Flächenformen ist die fraktale Dimension $(F R A C)$. Sie basiert auf den Untersuchungen von Mandelbrot (1983). Dieser entwickelte die fraktale Analyse zur Beschreibung räumlicher Phänomene auf mehreren Skalenebenen, welche kontinuierlich, aber nicht differenzierbar sind (Krummel et al., 1987). Die fraktale Dimension kann für Flächen Werte zwischen 1 (einfache Formen) und 2 (unregelmäßige Formen) annehmen (McGarigal et al., 2002).

$$
F R A C=\frac{2 * \ln (0.25 * p)}{\ln (a)}
$$

Waldrandlänge(EDGE, TOTAL_EDGE) Die Randlänge beschreibt auf Patchebene den Umfang eines Patches. Auf Landschaftsebene kann sie als mittlerer Umfang aller Patches $(E D G E)$ oder als Summe der Umfänge aller Patches (TOTAL_EDGE) angegeben werden.

Tabelle 1.: Ausgewählte Landschaftsstrukturmaße, die verschiedenen ökologischen Prozessen zugeordnet werden können und im Rahmen der Simulation untersucht werden.

\begin{tabular}{lll}
\hline Landschaftscharakteristik & Ökologischer Prozess & Landschaftsstrukturmaß \\
\hline Waldanteil & Extinktion & COVER \\
Patchgröße & Habitat Eignung & SIZE \\
Patchanzahl & Migration & N_PATCH \\
Patchform & Randeffekte & SHAPE, PARA, FRAC \\
Randlänge & Randeffekte & EDGE, TOTAL_EDGE \\
\hline
\end{tabular}




\section{Simulationsmethoden}

\subsection{Erstellung räumlicher Landschaftsmodelle}

Um Landschaften simulieren zu können, müssen zunächst Modelle zur Beschreibung der landschaftsbildenden Prozesse gefunden werden. Ein Modell, das sehr häufig in der Landschaftsökologie verwendet wird, ist das neutrale Modell. Es unterstellt eine zufällige Verteilung der Landschaftselemente im Raum. Tobler (1970) formulierte das erste Gesetz der Geographie, welches besagt, dass benachbarte Objekte ähnlicher sind als entfernte. Viele Untersuchungen bestätigen, dass die meisten ökologischen Prozesse starke räumliche Abhängigkeit aufweisen. Daher erscheinen Modelle, die eine räumliche Abhängigkeit berücksichtigen, vorteilhafter als neutralen Modelle. Die Implementierung von räumlichen Abhängigkeiten kann mit Hilfe von stochastischen Modellen erfolgen. Dabei stellt ein räumlicher stochastischer Prozess zunächst eine Menge an Zufallsvariablen $Z$, die über die Dimensionen $D \subset \mathbb{R}^{d}$ indexiert werden können (Schabenberger \& Gotway, 2005). Übertragen auf die Landschaftssimulation lässt sich dann eine Landschaft als Menge von stochastischen Prozessen in einem $d>1$ dimensionalen Raum darstellen. Solche Modelle werden Zufallsfelder genannt.

Zwei Eigenschaften der Zufallsfelder sind für die Modellierung von Landschaften von Bedeutung. Zum einen die Verteilung der $Z$-Variablen, zum anderen ihre räumliche Variation. Da die landschaftsbildenden Prozesse häufig unbekannt sind, wird zunächst eine Gaußverteilung angenommen. Ein Zufallsfeld $\left\{Z_{(s)}: s \in D \subset R^{d}\right\}$, dessen kumulative Häufigkeitsverteilung einer Gaußverteilung folgt, wird als Gauß'sches Zufallsfeld bezeichnet. Neben der Verteilung der Werte ist die Stärke der räumlichen Abhängigkeit für die Landschaftsmodelle von großer Bedeutung. Eine Möglichkeit zur Beschreibung solcher Phänomene ist die räumliche Autokorrelation. Wird $Z_{s}$ als Realisierung eines stochastischen Prozesses am Ort $s(x, y)$ verstanden, so beschreibt die Autokorrelation die Korrelation der Werte $Z_{s i}$ und $Z_{s j}$ (Schabenberger \& Gotway, 2005). Wenn diese Vorstellung in ein dreidimensionales Koordinatensystem mit $Z$ auf der senkrechten Achse übertragen wird, ergeben sich bei positiver Autokorrelation flächenartige Punktwolken, da die Wahrscheinlichkeit, dass näher beieinander liegende Werte ähnlicher sind, größer ist (Schabenberger \& Gotway, 2005). Nimmt man des Weiteren an, dass diese Abhängigkeit in der gesamten Landschaft konstant ist, wird von einem stationären stochastischen Prozess gesprochen. In diesem Fall wird die Autokorrela- 
tion nicht mehr durch die absolute Lage, sondern nur noch durch die Distanz $h$ zwischen den Punkten bestimmt. Die räumliche Autokorrelation kann als Kovarianz des selben Attributes an zwei verschiedenen Orten ausgedrückt werden. Die Simulation von Landschaften mit räumlicher Abhängigkeit kann dann mit Hilfe von Kovarianzmodellen erfolgen. Für die vorliegende Untersuchung war es wichtig, die Fragmentierung der Landschaft variieren zu können. Geht man davon aus, dass die räumliche Abhängigkeit der Landschaft als Indikator für die Fragmentierung genutzt werden kann, deutet eine geringe Autokorrelation auf stärker fragmentierte Landschaften hin. Ein Kovarianzmodell, das die Variation der räumlichen Autokorrelation ermöglicht, ist die einparametrische Form des Cauchy-Modells (siehe Gleichung 5).

$$
C(h)=\left(1+|h|^{\alpha}\right)^{\frac{1}{\alpha}}
$$

In diesem Modell ist $\alpha$ der einzige Parameter. Er beschreibt die fraktale Dimension $F$, welche sich nach $F=3-\alpha / 2$ aus $\alpha$ ableiten lässt (Gneiting \& Schlather, 2004). Die fraktale Dimension beschreibt die Komplexität einer geometrischen Form (Mandelbrot, 1983). Der $\alpha$ Wert beeinflusst daher direkt die räumliche Abhängigkeit innerhalb der Landschaftsmodelle. Kleine Werte stehen für eine schwache und große $\alpha$ Werte für eine starke Autokorrelation.

Für die Simulation der Kronenkarten mit starker Fragmentierung wurde ein Wert von $\alpha=0.7$ und für wenig fragmentierte Landschaften $\alpha=1.4$ gewählt. Um die Kronenkarten zu erstellen, mussten die kontinuierlichen Zufallsfelder in eine binäre Karte überführt werden. Neben der Fragmentierung soll der Baumkronenanteil der Landschaften variiert werden. Aufgrund der Gauß'schen Verteilung der Werte konnten mit Hilfe der 0.3 und 0.7 Quartile für jedes Modell zwei Schwellenwerte bestimmt werden. Diese Schwellenwerte wurden auf die kontinuierlichen Gauß'schen Felder angewendet, wodurch Landschaften mit 30\% oder $70 \%$ Baumkronenanteil entstanden. Durch die Variation der beiden exogenen Faktoren Fragmentierung und Baumkronenanteil mit jeweils zwei Faktorstufen ergaben sich vier Grundtypen der Kronenkarten, welche in Abbildung 1 dargestellt sind.

Neben den einfachen planimetrischen sollten auch dreidimensionale Landschaftmodelle untersucht werden. Diese unterscheiden sich insofern, als dass sie für jeden Punkt neben der Information der Landbedeckung(Baumkrone/Nicht-Baumkrone) auch eine Höheninformation enthalten. Diese Höheninformation wird üblicherweise in Form von digitalen Gelände- 


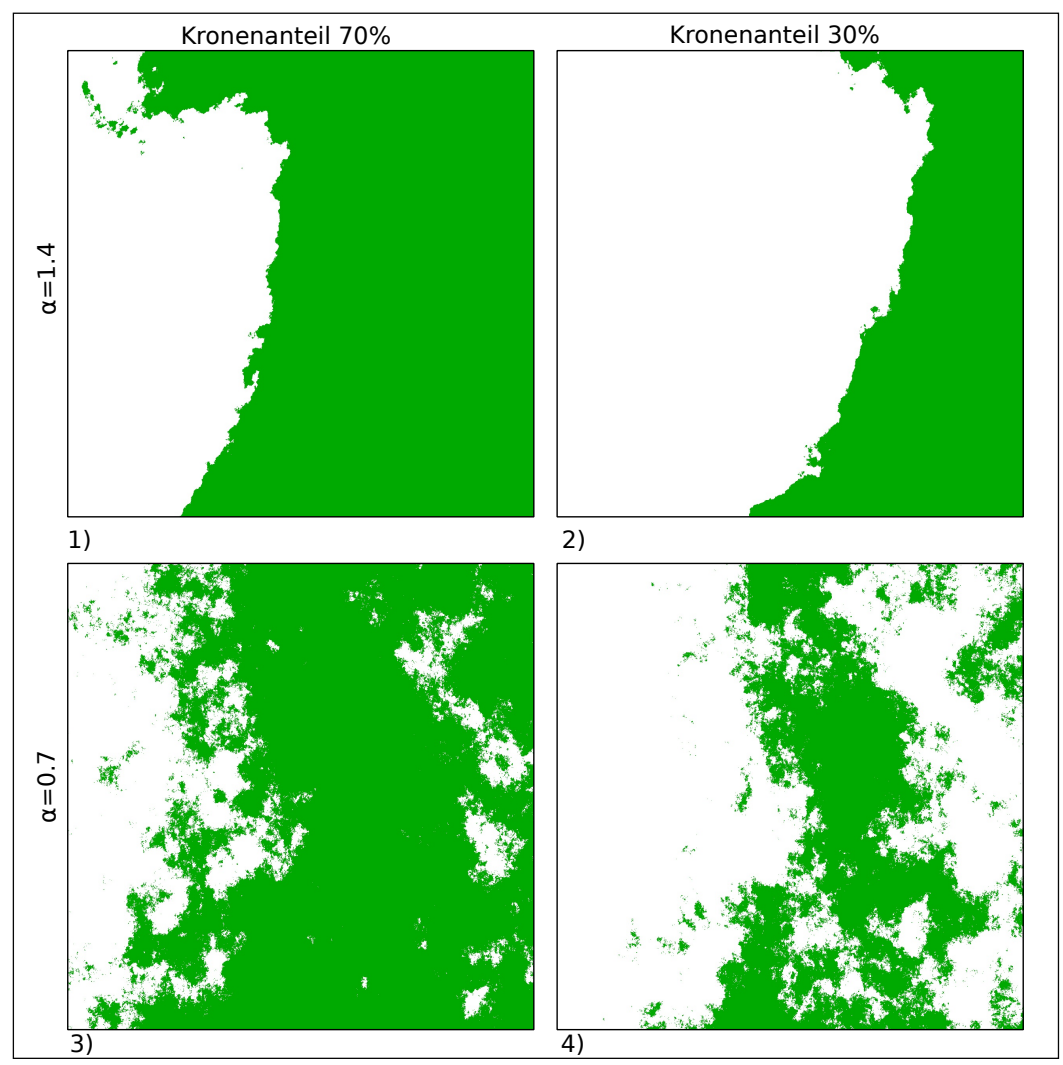

Abbildung 1.: Beispiel der vier Grundtypen von Baumkronenkarten (grün=Baumkronen, wei $\beta=$ Nicht-Baumkronen) mit 1) einer kompakten Landschaft mit hohem Baumkronenanteil 2) einer kompakten Landschaft mit geringem Baumkronenanteil 3) einer stark fragmentierten Landschaft mit hohem Baumkronenanteil und 4) einer stark fragmentierten Landschaften mit geringem Baumkronenanteil. Quelle: Magdon \& Kleinn (2012), verändert.

modellen (DGM) gespeichert. In der vorliegenden Arbeit werden, neben den Baumkronenkarten, auch die DGM mit Gauß'schen Feldern $(\alpha=0.7)$ simuliert. Der Wertebereich der Gauß'schen Felder wurde durch eine lineare auf 0 - 1000 gestreckt und auf ganze Zahlen gerundet, um realistische Landschaftsmodelle mit Höhenunterschieden zwischen 0 und 1000m abzubilden. Durch Überlagerung der Baumkronenkarten mit den Geländemodellen wurden die dreidimensionalen Landschaftsmodelle erstellt. 


\subsection{Variation der Beobachtungsskalen}

Zwei endogene Faktoren, welche Aspekte der Beobachtungsskala beschreiben, sollen untersucht werden. Zum einen die horizontale räumliche Auflösung der Baumkronenkarten und zum anderen die vertikale Auflösung der dreidimensionalen Geländemodelle. Zur Abbildung verschiedener horizontaler Auflösungen wurden die binären Baumkronenkarten mit einem Filter, der jeweils $9 \times 9 p x$ zusammenfasst und den häufigsten Wert in der neuen gröberen Karte speichert, vergröbert. Die Verwendung einer ungeraden Filtergröße erlaubt in allen Fällen eine eindeutige Mehrheit zu bestimmen. Die Festlegung der räumlichen Auflösung der hochaufgelösten Karten auf 1m geschah willkürlich und hat keine Auswirkung auf die Ergebnisse. Es erleichtert jedoch die Darstellung und Kommunikation. Als höchste Auflösung wurden Baumkronenkarten mit $2700 \times 2700 p x$ verwendet. Diese wurden anschließend auf $300 \times 300 p x(9 \mathrm{~m})$ reduziert. Die Simulation zweier vertikaler Auflösungen erfolgte durch Aggregation der ganzzahligen Höhenwerte (1m Auflösung) in 25m Stufen.

\subsection{Waldflächenkartierung auf Basis von Überschirmungsgradienten}

Bei der Kartierung der Waldflächen sollen zwei Kriterien variiert werden: die geforderte Mindestüberschirmung und die Waldranddefinition. Zur Definition des Waldrandes soll hier das Referenzflächenverfahren genutzt werden. Für die Simulation wird die Waldranddefinition daher durch Variation der Größe der Referenzfläche abgebildet. Somit ergeben sich zwei Faktoren: i) die Mindestüberschirmung und ii) die Größe der Referenzfläche, für welche die Effekte auf die Bestimmung des Waldanteils untersucht werden. Zur Implementierung des Referenzflächenverfahrens bei der Auswertung der simulierten Karten wurde das in Abbildung 2 dargestellte Schema genutzt.

Für die Simulation wird angenommen, dass die simulierten Baumkronenkarten eine fehlerfreie Klassifizierung von Satellitenbildern darstellen, eine Annahme, die in der Praxis häufig nicht zutrifft. Hier wird sie jedoch getroffen, da Klassifikationsfehler in der Simulation nicht berücksichtigt werden sollen. Die Beschreibung der praktischen Bildklassifikation von Satellitenbildern und der damit verbundenen Fehler erfolgt im vierten Teil der Arbeit.

Zur Implementierung des Referenzflächenverfahrens wurden Moving-Window-Operatoren 


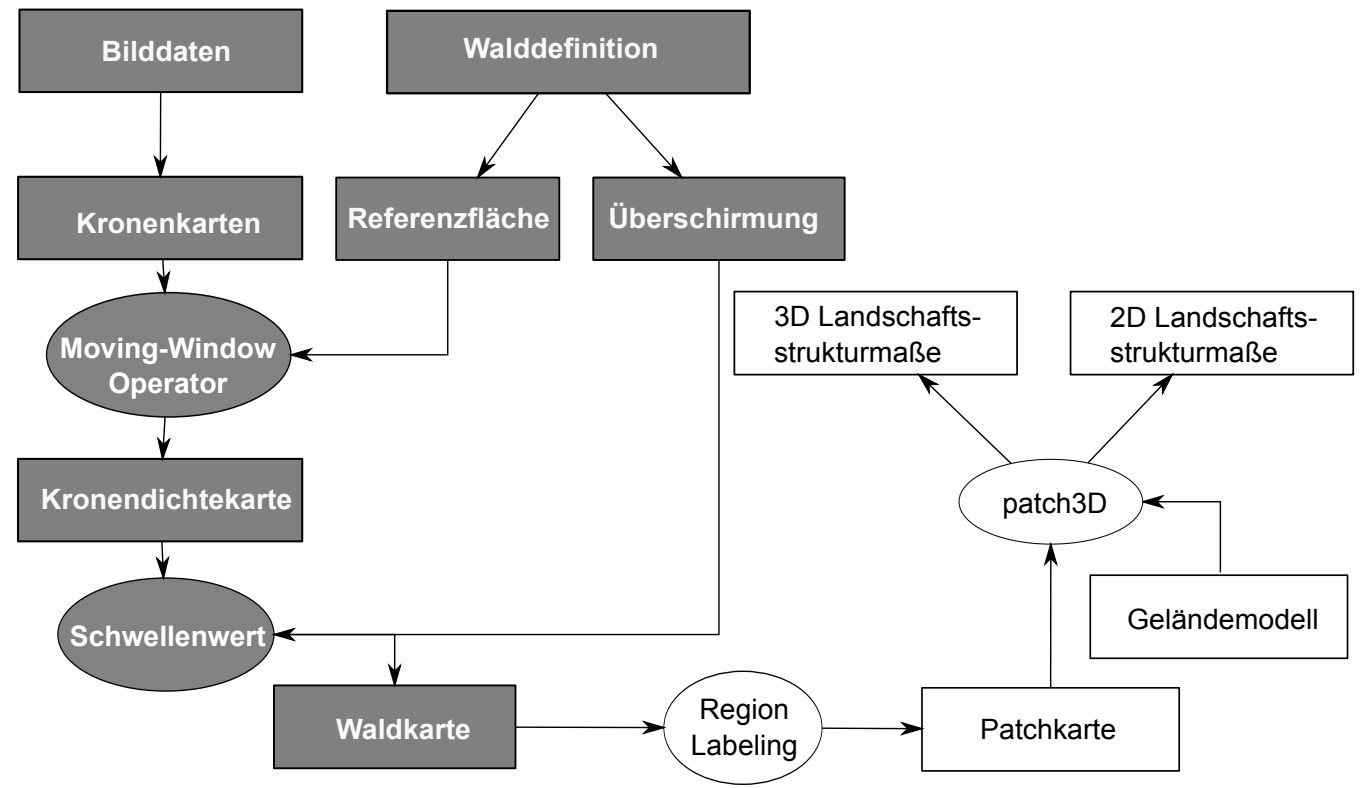

Abbildung 2.: Ablaufplan zur Anwendung des Referenzflüchenverfahrens zur Überführung der Baumkronen- in Waldflächenkarten (grau) mit anschließender Landschaftsstrukturanalyse (weiß). Quelle: Magdon \& Kleinn (2012), verändert.

eingesetzt. Diese führen ein Beobachtungsfenster Pixel für Pixel über das Ausgangsbild und speichern einen neuen Wert für das zentrale Pixel des Beobachtungsfensters ab. Dieser ergibt sich als Statistik der Pixelwerte innerhalb des Beobachungsfensters. Als Beobachtungsfenster werden dabei meist quadratische Flächen verwendet, wobei auch andere Formen möglich sind. Durch Anwendung des Moving-Window-Operators auf alle Pixel des Ausgangsbildes entsteht ein neues gefiltertes Bild. In den Randbereichen liegen die Beobachtungsfenster nicht vollständig im Bild, so dass sich die Statistik dann auf eine kleinere Nachbarschaft bezieht oder nicht berechnet werden kann. Diesem unerwünschten Effekt kann unter anderem durch das Abtrennen der betroffenen Randbereiche begegnet werden. Dadurch verringert sich die Anzahl der Spalten und Reihen des Bildes um eine Fensterbreite.

Die mit Hilfe der Moving-Window-Operatoren aus den Baumkronenkarten erzeugten Bilder werden im Folgenden als Baumkronendichtekarten bezeichnet, da sie bezogen auf die Größe des Beobachtungsfensters den Anteil der Baumkronenpixel angeben. Das Beobachtungsfenster des Moving-Window-Operators entspricht somit der Referenzfläche für die Bestimmung der Kronenüberschirmung. Zur Berechnung der Baumkronendichtekarten wurden zehn verschiedene quadratische Referenzflächengrößen $(9,49,121,255,361,529$, 
729, 961, 1225 und 1521px) verwendet. Dabei beziehen sich die angegebenen Pixelzahlen auf die niedrige Auflösungsstufe. Eine Referenzfläche von 9px entspricht dann einer Anzahl von 27px in der hohen Auflösungsstufe. Durch das Abtrennen der Randbereiche ergaben sich für verschiedene Referenzflächengrößen unterschiedliche Ausdehnungen der Baumkronendichtekarten. Wu (2004) zeigte, dass die Ausdehnung der zu untersuchenden Landschaft signifikanten Einfluss auf die LSM haben kann. Daher wurden alle Baumkronenkarten auf die kleinste Ausdehnung zugeschnitten, was eine einheitliche Größe der Karten sicherstellte. Aus den Baumkronenkarten wurden im nächsten Schritt, durch Anwendung von zehn verschiedenen Schwellenwerten für die Überschirmung $(10 \%, 20 \% \ldots 100 \%)$, binäre Waldkarten abgeleitet, die als Ausgangskarten für die Berechnung der LSM dienten.

Die Simulation, wie sie in Abbildung 2 dargestellt ist, wurde mit der Statistik-Software $\mathrm{R}$ (R Development Core Team, 2011) programmiert. Für die Simulation der Baumkronenkarten wurde das R-Paket RandomFields (Schlather, 2001) verwendet. Die Moving-WindowOperatoren wurden mit Hilfe einer Schnittstelle zum Softwarepaket Geographic Resources Analysis Support System (GRASS Development Team, 2011) implementiert. Die Simulation konnte mit dem Message Passing Interface-Standard parallelisiert werden. Dafür wurde die OpenMPI Bibliothek verwendet. Die Prozessierung erfolgte auf einem Cluster von fünf Desktop-Computern mit insgesamt 24 Kernels.

\section{4. patch3D: Ein Programm zur dreidimensionalen Landschaftsanalyse}

Die Landschaftsanalyse der Waldkarten wurde in einer zweiten Phase der Bildauswertung, wie sie in der Abbildung 2 weiß dargestellt ist, durchgeführt. Im ersten Schritt der Landschaftsanalyse wurden die erzeugten binären Waldkarten in Patchkarten überführt. In der Patchkarte sind alle direkt miteinander verbundenen Baumkronenpixel als Patch gruppiert und mit einer eindeutigen ID versehen. Dazu wurde ein component labeling Algorithmus (Chang et al., 2004), welcher im R-Paket SDM-Tools (VanDerWal et al., 2011) implementiert ist, verwendet. Die Nachbarschaft wurde jeweils für alle acht umliegenden Pixel untersucht (8-Pixel-Neighborhood).

Die Landschaftsanalyse erfolgte sowohl für zwei- als auch für dreidimensionale Landschaftsmodelle. Als Grundlage für die Berechnung der dreidimensionalen Oberfläche diente 
die von Jenness (2004) entwickelte Triangulationsmethode, die von Hoechstetter et al. (2008) um die Berechnung der dreidimensionalen Umfänge erweitert wurde. Die Berechnung der dreidimensionalen Oberfläche erfolgt in drei Schritten, die im Folgenden erläutert werden und in Abbildung 3 dargestellt sind.

\begin{tabular}{|l|l|l|}
\hline 190 & 170 & 155 \\
\hline 183 & 165 & 145 \\
\hline 175 & 160 & 122 \\
\hline
\end{tabular}

1)

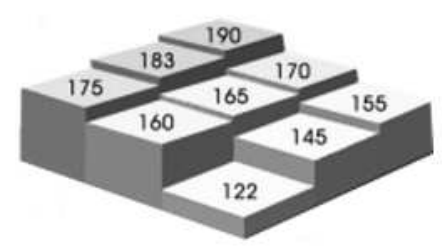

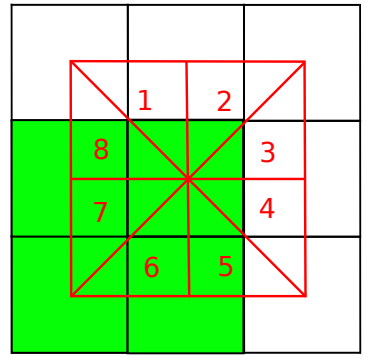

2)

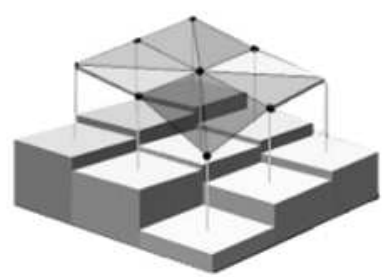

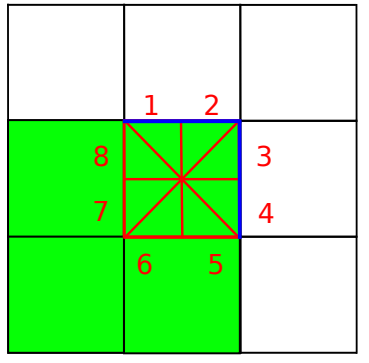

3)

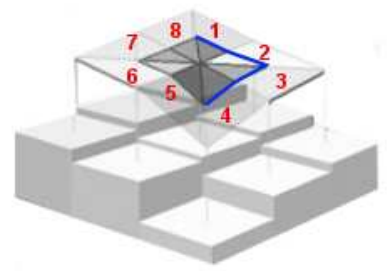

Abbildung 3.: Darstellung der dreidimensionalen Oberflächenberechnung in drei Schritten mit 1) Extraktion der neun Höhenwerte und Patch-ID's 2) Triangulation mit acht Dreiecksflächen und 3) Reduktion der Oberflächen auf die Pixelgröße. Die blaue Linie stellt den Waldrand des Pixels dar. Quelle: Jenness (2004), verändert.

\section{Extraktion}

Im ersten Prozessierungsschritt erfolgt die Koregistrierung des Geländemodells und der Patchkarte. Auf die Patchkarte wird anschließend ein Moving-Window-Operator mit $3 \times 3 p x$ angewendet. Die Korrektur des Randproblems erfolgt, im Gegensatz zum Ansatz bei der Waldflächenkartierung, durch Spiegelung. Dafür werden die Landschaftsmodelle um zwei Zeilen und Spalten erweitert, indem jeweils die erste und letzte Zeile und Spalte wiederholt werden. Der Moving-Window-Operator extrahiert dann für jedes Pixel jeweils neun Höhenwerte aus dem Geländemodell und neun Patch ID's aus der Patchkarte. 
2. Triangulation

Im zweiten Arbeitsschritt werden aus den neun Höhenwerten und der Pixelgröße insgesamt acht rechtwinklige Dreiecke gebildet und die jeweiligen Kantenlängen auf Grundlage des Satz des Pythagoras berechnet. Auf Basis der drei Seitenlängen wird anschließend die Dreiecksfläche bestimmt und für alle acht Dreiecke aufsummiert. Für jedes Pixel wird geprüft ob und wie viele der vier Kanten des Pixels Waldränder sind, sich also in direkter Nachbarschaft zu Nicht-Waldflächen befinden. Durch aufsummieren der dreidimensionalen Kantenlängen wird die Waldrandlänge pro Pixel berechnet.

\section{Reduktion}

Im letzten Arbeitsschritt werden die Dreiecksflächen und Waldrandlängen auf die Ausdehnung des zentralen Pixels reduziert. Abschließend werden die Werte pro Patch aggregiert um sowohl die zwei- als auch die dreidimensionalen Flächen und Umfänge pro Patches zu bestimmen. Aus diesen werden dann die LSM SHAPE, FRAC und EDGE, wie sie in Abschnitt 5.2 beschrieben sind, als zwei- und dreidimensionale Metriken berechnet.

Die Berechnung von LSM auf Basis von dreidimensionalen Landschaftsmodellen ist ein relativ neuer Ansatz, weshalb nur wenige Softwarepakete für die Auswertung zur Verfügung stehen. Die am weitesten fortgeschrittene Implementierung LandscapeMetrics-3D wurde von Walz et al. (2010) als Erweiterung der Software ArcGis-Desktop der Firma Environmental Systems Research Institute (ESRI) entwickelt und bietet umfangreiche Funktionen zur Berechnung von LSM auf Basis von dreidimensionalen Landschaftsmodellen. Aufgrund der Programmstruktur von ArcGIS und der damit verbundenen Speicherverwaltung ist die Berechnung großer Landschaftsmodelle (z. B. $2700 \times 2700 p x)$ jedoch nicht möglich. Außerdem bietet sie keine Funktion zur automatischen Prozessierung mehrerer Landschaften (Batchmodus), wie es die vorliegende Simulation erfordert. Um die angestrebten Landschaftsanalysen durchführen zu können, war es daher notwendig eine eigene Software zu programmieren. Dafür wurde das Programm patch3D erstellt, welches die von Walz et al. (2010) entwickelten Methoden in der Programmiersprache $C^{++}$umsetzt und somit eine hohe Geschwindigkeit und effiziente Speicherverwaltung gewährleistet. Die Ergebnisse werden von dem Programm in einer Textdatei gespeichert und stehen dann für die weitere Auswertung zur Verfügung. Der patch3D Quellcode wurde unter der freien Lizenz GNU GPL v3 veröffentlicht (Magdon, 2011). 


\subsection{Versuchsdesign}

Im Rahmen der Simulation soll die Wirkung von drei Aspekten des Monitoringdesigns (endogene Faktoren: Wald- und Waldranddefinition, Beobachtungsskala und Landschaftmodelle) auf die Waldflächen- und Strukturbeschreibung untersucht werden. Dabei werden sowohl die direkten Wirkungen als auch die Wechselwirkungen mit Landschaftseigenschaften (exogene Faktoren: Fragmentierungszustand und Baumkronenanteil) untersucht. Letztendlich sollen die sechs Faktoren hinsichtlich ihrer Wirkung auf die Waldflächenerfassung und deren Interaktion geprüft werden, wie sie in Tabelle 2 zusammengefasst sind.

Tabelle 2.: Zusammenfassung der Faktoren und Faktorstufen, die anhand der Simulation untersucht werden.

\begin{tabular}{lll}
\hline Typ & Faktor & Faktorstufen \\
\hline \multirow{2}{*}{ Endogen } & Walddefinition (Baumkronenüberschirmung) & $t \in\{0.1,0.2, \ldots, 1\}$ \\
& Waldranddefinition (Referenzfläche) & $s \in\{9,49, \ldots, 1521\}$ \\
& Landschaftsmodell & $2 \mathrm{D} / 3 \mathrm{D}$ \\
& Beobachtungsskala (horizontale Auflösung) & $h \in\{0 ; 9\}$ \\
& Beobachtungsskala (vertikale Auflösung) & $v \in\{1 ; 50\}$ \\
\hline \multirow{2}{*}{ Exogen } & Komposition (Baumkronenanteil) & $p \in\{0.3 ; 0.7\}$ \\
& Konfiguration (Fragmentierung) & $\alpha \in\{0.7 ; 1.4\}$ \\
\hline
\end{tabular}

Für die Simulation wurden jeweils $n=100$ unabhängige Zufallsfelder der vier Grundtypen in zwei horizontalen Auflösungsstufen $h$ simuliert, so dass $n=800$ Kronenkarten entstanden. Aus diesen wurden mit zehn Referenzflächengrößen $s, n=8000$ Kronendichtekarten abgeleitet. Die Kronendichtekarten wurden anschließend mit zehn verschiedenen Schwellenwerten für die Überschirmung $t$ in $n=80000$ Waldkarten überführt. Die Berechnung der LSM erfolgte dann für das zwei- und dreidimensionale Landschaftsmodell, wobei die dreidimensionalen Landschaftsmodelle zusätzlich mit zwei vertikalen Auflösungsstufen $v$ betrachtet wurden. Somit ergaben sich $80000 \times 3=240000$ Landschaftsmodelle, für welche die in Tabelle 1 angegebenen LSM berechnet wurden. Die Berechnung der Kronendichtekarten mit dem Moving-Window-Operator ist besonders für große Beobachtungsfenster/Referenzflächen sehr rechenintensiv, da über alle Pixel im Bild iteriert werden muss. Daher ist die Anzahl von $n=100$ Wiederholungen im Wesentlichen durch die für die Simulation zur Verfügung stehenden Ressourcen und nicht durch statistische Überlegungen bestimmt. 



\section{Ergebnisse der Simulation}

\subsection{Landschaftssimulation}

Als Grundlage für die Untersuchungen dienten auf Basis der Gauß'schen Zufallsfelder generierte binäre Kronenkarten. Es wurden jeweils $n=100$ Kronenkarten für die vier Grundtypen erzeugt. Die Qualität der so erzeugten Landschaften lässt sich nur schwer bemessen und kann letztendlich nur optisch beurteilt werden. Die in Abbildung 1 dargestellten Beispiele der Kronenkarten ergeben realistisch wirkende Landschaften mit klaren Unterschieden zwischen den vier Grundtypen. In Tabelle 3 sind die gemittelten Kenngrößen für die Grundtypen auf Landschaftsebene zusammengefasst. Dabei zeigt sich, dass der $\alpha$-Wert des einparametrischen Cauchy-Kovarianzmodells (siehe Gleichung 6.1) im wesentlichen die Anzahl der Patches beeinflusst. Für $\alpha=0.7$ können deutlich höhere Patchzahlen beobachtet werden. Die Anwendung der entsprechenden Quantile als Schwellenwert ermöglicht die Variation der Kronenanteile. Durch das Abschneiden der Ränder entsprechen die Kronenanteile jedoch nicht exakt den angestrebten Quantilen $(0.3,0.7)$, da die Ränder erst nach der Schwellenwertsetzung abgeschnitten wurden.

Tabelle 3.: Kenngrößen der Patches aus den simulierten Landschaften mit höchster Auflösung, gemittelt über $n=100$ Wiederholungen der vier Grundtypen. Angegeben sind die Werte für die zweidimensionalen Karten, wobei Patchanzahl und Umfang aufsummiert und Größe, FRAC und SHAPE gemittelt wurden.

\begin{tabular}{rrrrrrr}
\hline$\alpha$-Wert & Kronenanteil & Anzahl & Größe (ha) & Umfang $(\mathrm{km})$ & FRAC & SHAPE \\
\hline 1.40 & 0.30 & 229.90 & 0.66 & 21.12 & 1.38 & 1.21 \\
1.40 & 0.74 & 177.14 & 2.33 & 19.15 & 1.39 & 1.20 \\
0.70 & 0.32 & 9065.40 & 0.01 & 320.30 & 1.40 & 1.25 \\
0.70 & 0.75 & 6279.23 & 0.05 & 281.35 & 1.40 & 1.22 \\
\hline
\end{tabular}

Wie erwartet ergaben sich für niedrigere $\alpha$-Werte, also stärker fragmentierte Landschaften, geringere Patchgrößen, höhere Patchanzahlen und damit verbunden ein höherer mittlerer Umfang. Die Metriken SHAPE, FRAC zeigen für stärker fragmentierte Landschaften geringere Werte. Allerdings sind die Unterschiede sehr klein. Insgesamt scheint die Charakteristik der künstlichen Landschaften für die weiteren Simulationen geeignet, da die angestrebten 
Variationen der Landschaftskonfiguration und -komposition gut abgebildet werden und die Karten realistisch wirken.

\subsection{Geometrische Analyse des Referenzflächenverfahrens}

Die Waldranddefinition ist von zentraler Bedeutung für die Ausweisung von Waldflächen. In der vorliegenden Arbeit wurde das Referenzflächenverfahren eingesetzt, welches zwei Kriterien, die Referenzfläche und die Mindesüberschirmung, kombiniert. Zur Analyse der Effekte beider Kriterien auf die Lage des Waldrandes wurden zunächst geometrische Analysen für zwei Waldrandmodelle durchgeführt (siehe Abbildung 4).
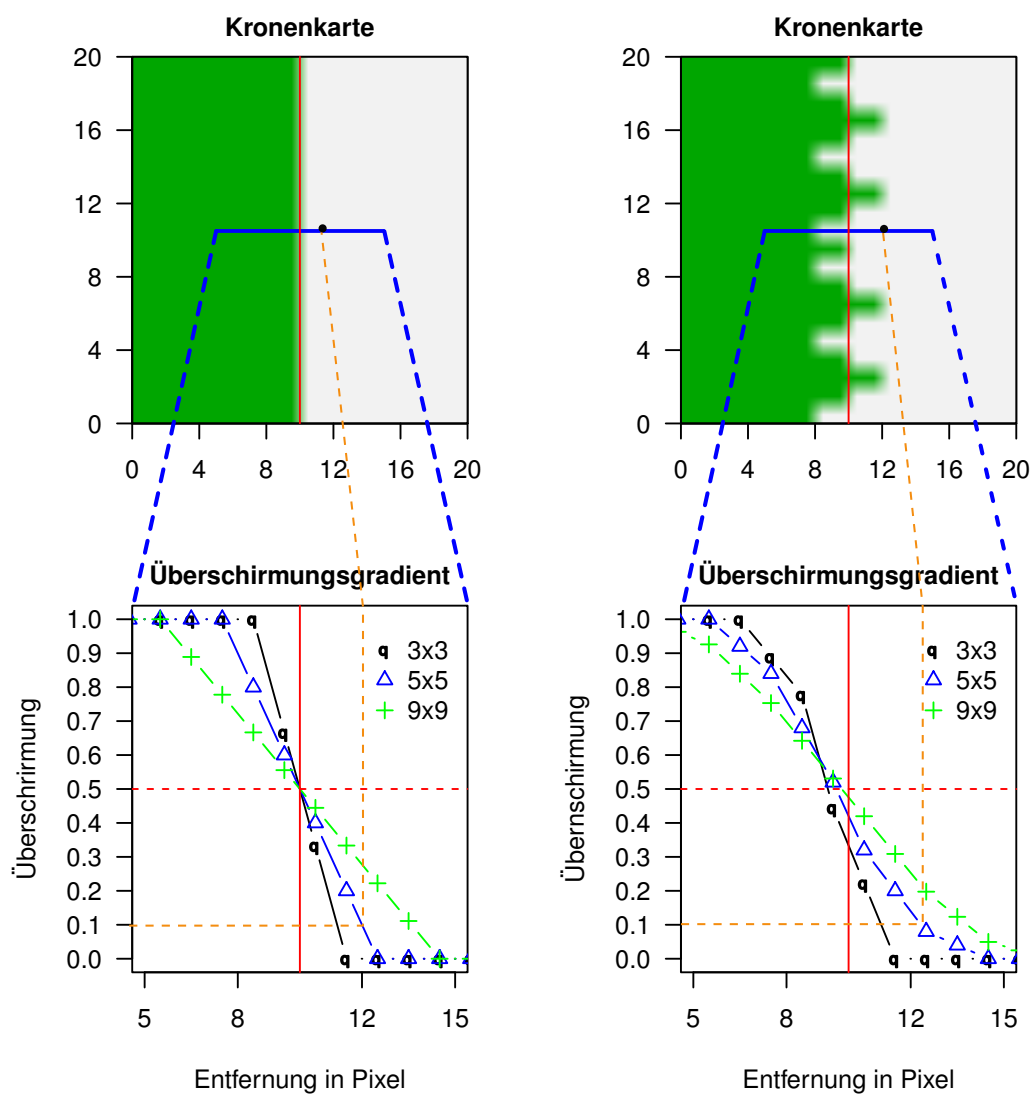

Abbildung 4.: Schematische Darstellung der Überschirmungsgradienten auf einem Transekt (blaue Linie) entlang eines regelmäßigen (links) und unregelmäßigen (rechts) Waldrandes für drei Referenzflächengrößen. Quelle Magdon \& Kleinn (2012), verändert. 
Im linken Teil der Abbildung 4 markiert eine gerade Linie den Übergang zwischen den von Baumkronen überschirmten zu den nicht-überschirmten Flächen. Wird die Überschirmung entlang eines orthogonal zum Kronentrauf verlaufenden Transektes (blaue Linie) untersucht, so ergibt sich ein Überschirmungsgradient wie er im unteren Bildteil für drei verschiedene Größen der Referenzfläche dargestellt ist. Anhand dieses Gradienten kann die Lage des Waldrandes für verschiedene Referenzflächen und Mindestüberschirmungen ermittelt werden. In Abbildung 4 ist das beispielhaft für eine Mindestüberschirmung von 10\% $(t=0.1)$ und eine Referenzfläche $s$ von $5 \times 5 p x$ dargestellt (orange Linie). Der Waldrand verschiebt sich in diesem Beispiel um ungefähr 2px von 10 nach 12. Ein Vergleich der unterschiedlichen Referenzflächengrößen zeigt, dass die Steigung der Gradienten von der Größe der Referenzfläche abhängt. Kleine Referenzflächen zeigen steile und große Referenzflächen flache Gradienten. Für den geradlinigen Kronentrauf (linke Seite) ergeben sich lineare Gradienten mit einem gemeinsamen Schnittpunkt bei $t=0.5$. In diesem Fall verschiebt sich der Waldrand für $t<0.5$ in den nicht überschirmten und für $t>0.5$ in den überschirmten Bereich. Die horizontale Distanz, um welche der Waldrand verschoben wird, hängt dabei von der Größe der Referenzfläche $s$ und der Mindestüberschirmung $t$ ab. Bemerkenswert ist, dass für $t=0.5$ der Waldrand, unabhängig von $s$, genau auf der Kronentraufe liegt. In diesem speziellen Fall spielt die Größe der Referenzfläche offenbar keine Rolle. Auch für den unregelmäßig verlaufenden Kronentrauf (rechte Seite) kommt es -in Abhängigkeit von der Größe der Referenzfläche und der gewählten Mindestüberschirmung- zu einer Verschiebung des Waldrandes. Im Unterschied zum geradelinigen Kronentrauf folgt der Überschirmungsgradient jedoch keiner Geraden sondern einer s-förmigen Kurve, welche für kleine Referenzflächen steiler und für große Referenzflächen flacher ist. Ein gemeinsamer Schnittpunkt der Gradienten, wie beim geradlinigen Kronentrauf, existiert nicht.

Anhand dieser geometrischen Darstellung des Waldrandes wird klar, dass die Variation der Referenzfläche und der Mindestüberschirmung zu einer Verschiebung der Waldränder und damit zu einer Änderung der Waldfläche führen kann. Dabei bestimmt $s$ maßgeblich die Distanz zwischen Kronentraufe und Waldrand und $t$ die Richtung, in welche der Waldrand verschoben wird. Prinzipiell ergibt sich für Schwellenwerte $t<0.5$ eine Vergrößerung und für Werte $t>0.5$ eine Reduktion der Waldfläche gegenüber der Kronenfläche. Dabei liegt der Kipppunkt bei geradlinigem Kronentrauf exakt bei 0.5. Bei unregelmäßigem Verlauf ist kein gemeinsamer Schnittpunkt vorhanden. In diesem Fall ist die Modellierung der Verschiebung schwierig, da der Verlauf der Gradienten von der Lage jedes einzelnen Pixels in der Referenzfläche abhängt. 


\subsection{Auswertung der Simulation}

\subsubsection{Einfluss der Wald- und Waldranddefinition}

Die schematische Darstellung der Waldränder in Abbildung 4 zeigte, dass die Mindestüberschirmung und die Referenzfläche erhebliche Wirkung auf die erstellten Waldkarten haben können. Auf Grundlage der simulierten Landschaften wurde geprüft, wie sich die Effekte der Waldrandverschiebung in komplexen Landschaften mit vielen Waldpatches auf die Waldfläche und Struktur auswirken. In Abbildung 5 wird dies beispielhaft für eine Landschaft anhand der Bildprozessierungskette veranschaulicht.

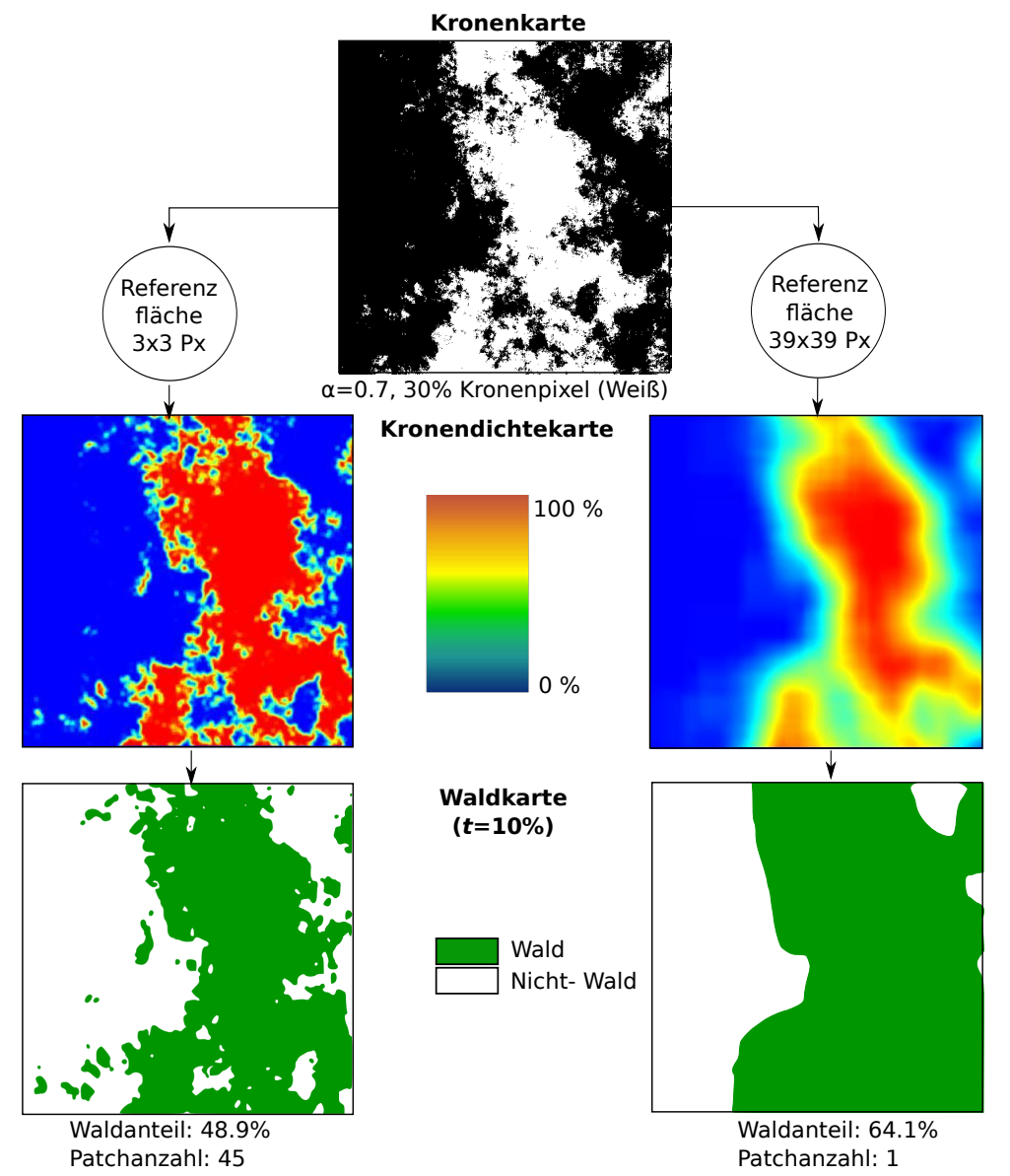

Abbildung 5.: Beispiel der Waldflächenkartierung für eine stark fragmentierte Landschaft ( $\alpha=0.7$ ) mit 30\% Kronenanteil und einer Mindestüberschirmung von $t=0.1$. Quelle: Magdon \& Kleinn (2012), verändert. 
Ausgehend von der simulierten Kronenkarte wurden Kronendichtenkarten für zwei unterschiedlich große Referenzflächen erstellt, die durch Anwendung eines Schwellenwertes, hier $t=0.1$ Mindestüberschirmung, in binäre Waldkarten überführt wurden. Die so generierten Waldkarten unterscheiden sich deutlich, sowohl in ihrem Waldanteil als auch in der räumlichen Verteilung der einzelnen Waldflächen, so dass sich zwei sehr unterschiedliche Landschaftsbilder ergeben. Für die kleine Referenzfläche $(7 \times 7 p x)$ ergibt sich ein Waldanteil von 48.9\% verteilt auf 45 Patches und für die größere Referenzfläche ein Waldanteil von $64.1 \%$ mit nur noch einem Patch. Im gegebenen Beispiel führte die Vergrößerung der Referenzfläche zu einer Erhöhung des Waldanteils indem Lücken zwischen den Patches geschlossen wurden. Dadurch erhöhte sich der Waldanteil bei gleichzeitiger Reduktion der Patchanzahl, was ausschließlich auf die Variation der Referenzfläche und damit verbundenen Verschiebung der Waldränder zurückzuführen ist.
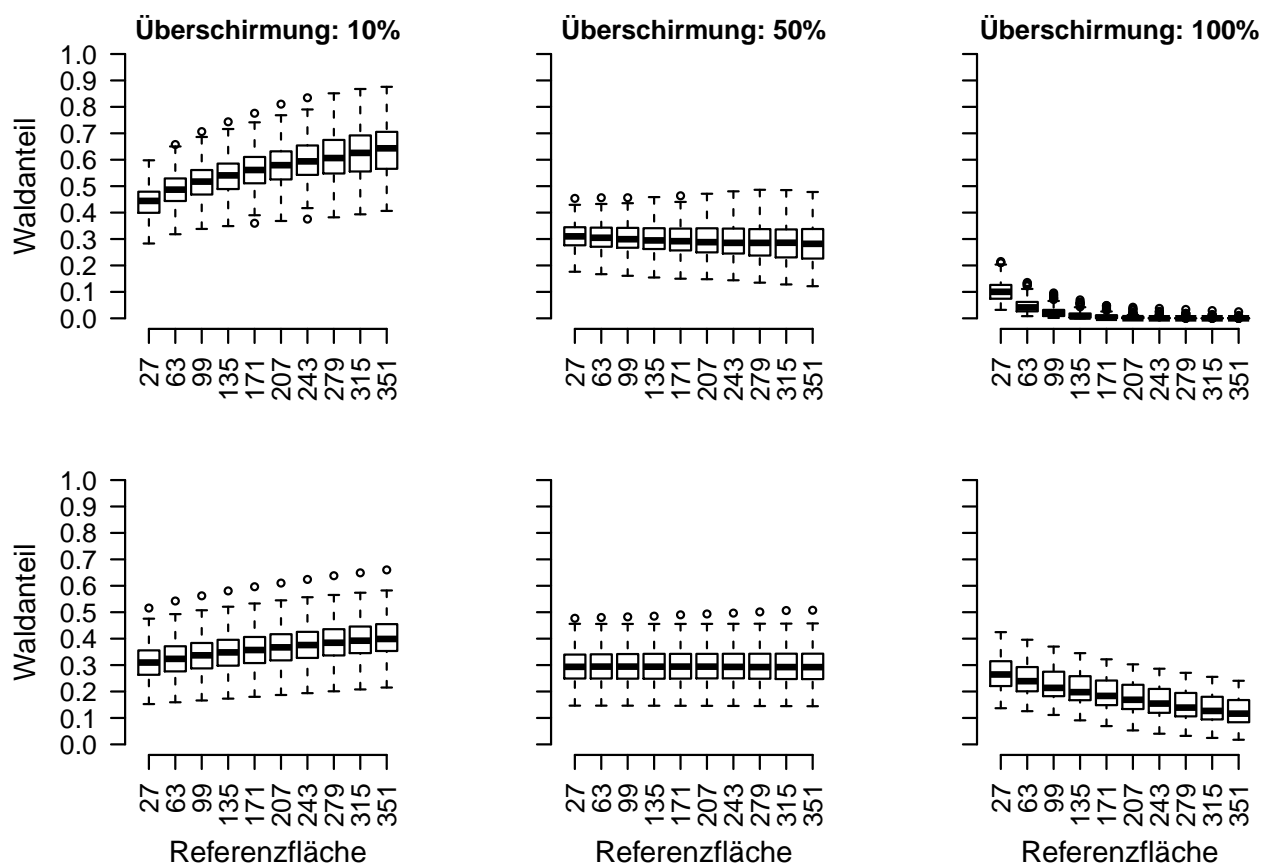

Abbildung 6.: Box-Whisker Plots der Waldanteile für verschiedene Referenzflächen und Mindestüberschirmungen dargestellt für $n=100$ stark fragmentierte $(\alpha=$ 0.7, obere Reihe) und gering fragmentierte Landschaften ( $\alpha=1.4$, untere Reihe) mit einem Kronenanteil von 30\%. Quelle: Magdon \& Kleinn (2012), verändert.

Abbildung 6 zeigt die Waldanteile für verschiedene Referenzflächen und Mindestüberschirmmungen für alle $n=100$ stark fragmentierten (obere Zeile) und kompakten (untere Zeile) 
Landschaften. Die Ergebnisse der Landschaftssimulation spiegeln die Beobachtungen des Waldrandmodells deutlich wieder. Für beide Fragmentierungszustände zeigten sich für $t=$ 0.5 nur geringe Unterschiede zwischen den Größen der Referenzflächen. In diesem Fall entsprach der mittlere Waldanteil ungefähr dem Kronenanteil der Landschaft (vergleiche Tabelle 3). Des Weiteren können zunehmende Waldanteile mit zunehmender Größe der Referenzfläche für $t<0.5$ und abnehmende Waldanteile für $t>0.5$ beobachtet werden, was auf die Verschiebung der Waldränder, wie sie bei der geometrischen Analyse in Abschnitt 7.2 beschrieben wurde, zurückgeführt werden kann. Dieser Trend verläuft bei kompakten Landschaften linear und bei stark fragmentierten nichtlinear, was ebenfalls durch das Waldrandmodell (für gerade Waldränder ergeben sich lineare und für unregelmäßige nichtlineare Überschirmungsgradienten), erklärt werden kann. Offensichtlich verlaufen die Waldränder in den kompakten Landschaften geradliniger als in stark fragmentierten. Insofern ist der $\alpha$-Wert der Kronenlandschaften ein Indikator für die Form des Kronentraufs, auch wenn sich die Formmetriken nur gering unterscheiden. Es ist zu erwähnen, dass die Form des Kronentraufs nur in Relation zur Referenzfläche beschrieben werden kann. Insofern sollte man davon sprechen, dass der Kronentrauf im Verhältnis zur Referenzfläche unregelmäßig verläuft.

Wie schon im Beispiel der Abbildung 5 veranschaulicht, wirkt die Wald-/Waldranddefinition neben dem Waldanteil auch auf die Landschaftskonfiguration, indem z. B. die Anzahl der Patches mit zunehmender Größe der Referenzfläche abnimmt. Dies kann darauf zurückgeführt werden, dass sich die Waldränder für große Referenzflächen so stark verschieben, dass benachbarte Flächen miteinander verbunden werden (siehe Abbildung $35 \mathrm{im}$ Anhang). Abbildung 7 zeigt eine Abnahme der Patchanzahl mit zunehmender Größe der Referenzfläche. Anders als beim Waldanteil sind keine Effekte der Mindestüberschirmung zu beobachten, daher kann -unabhängig vom gewählten Schwellenwert- eine Abnahme der Patchanzahl mit zunehmender Größe der Referenzfläche beobachtet werden. In stark fragmentierten Landschaften, wo wesentlich mehr Patches vorhanden sind, fällt die Patchanzahl exponentiell. In kompakten Landschaften ist häufig schon für kleine Referenzflächen nur eine Waldfläche vorhanden, so dass kein klarer Verlauf zu erkennen ist.

Wenn sich der Waldanteil bei zunehmender Größe der Referenzfläche für $t<0.5$ erhöht und die Patchanzahl abnimmt, müssen die mittleren Patchgrößen steigen, da mehr Wald auf weniger Patches verteilt wird. Abbildung 8 zeigt dies deutlich. Dabei ist zu beobachten, dass der Anstieg der Patchgröße nicht für alle Mindestüberschirmungen gleich ist. Wie vermutet steigt die mittlere Patchgröße für $t<0.5$ mit der Referenzfläche an. Für $t>0.5$ kann 

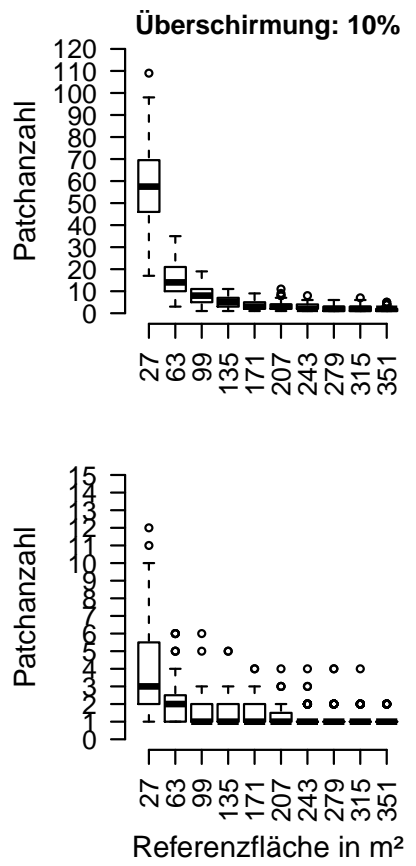
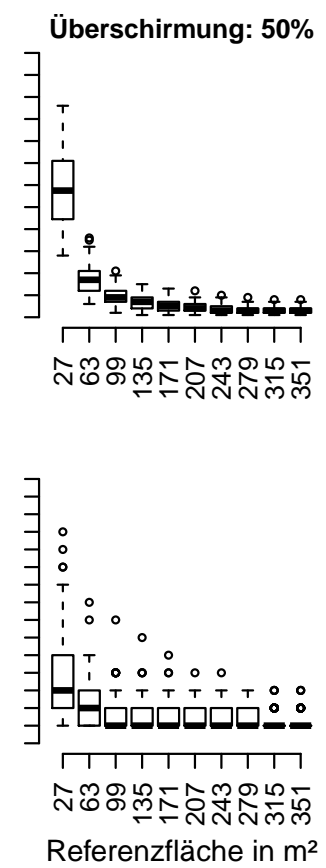
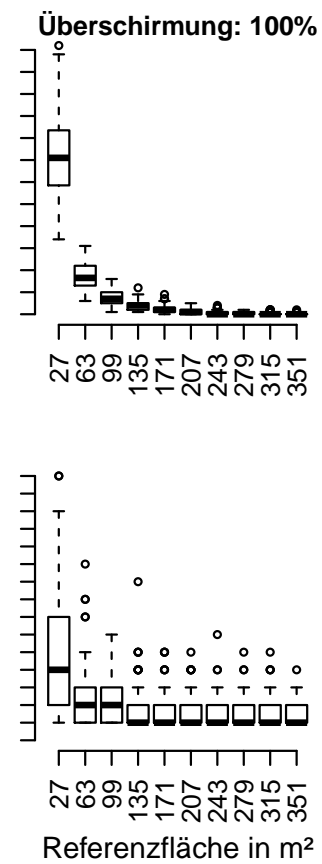

Abbildung 7.: Box-Whisker Plots Patchanzahlen für verschiedene Referenzflächen und Mindestüberschirmungen dargestellt für $n=100$ stark fragmentierte $(\alpha=0.7$, obere Reihe) und gering fragmentierte Landschaften $(\alpha=1.4$, untere Reihe) mit einem Kronenanteil von $30 \%$.

kein klarer Trend beobachtet werden. Hier nehmen die Waldflächen und die Patchzahlen proportional ab, so dass die Patchgrößen annähernd gleich bleiben.

Neben der Anzahl und der Größe ändert sich auch die Form der Patches in Abhängigkeit der Walddefinition. Dabei kann konstatiert werden, dass die Waldränder für große Referenzflächen regelmäßiger sind, da sich kleinere Ein- und Ausbuchtungen auflösen und scharfe Kanten abgerundet werden. So entsteht insgesamt ein kompaktere Waldfläche mit zunehmend runden Formen (siehe Abbildung 35 im Anhang). Diese Formänderungen wurden Anhand der LSM SHAPE, FRACT und EDGE für alle Patches analysiert und deren Werte für jede Waldkarte gemittelt. Abbildung 9 zeigt die Streuung der Mittelwerte für alle Karten, in denen mindestens eine Waldfläche vorhanden ist. Die SHAPE-Werte unterscheiden sich kaum und liegen im Mittel bei 1.5. Es ist kein systematischer Effekt der Referenzfläche zu beobachten. Auch sind kaum Unterschiede zwischen stark und gering fragmentierten Landschaften festzustellen. Für die FRAC Metrik ergibt sich sowohl für stark fragmentierte als auch für kompakte Landschaften eine geringe Abnahme der Werte mit zunehmender 

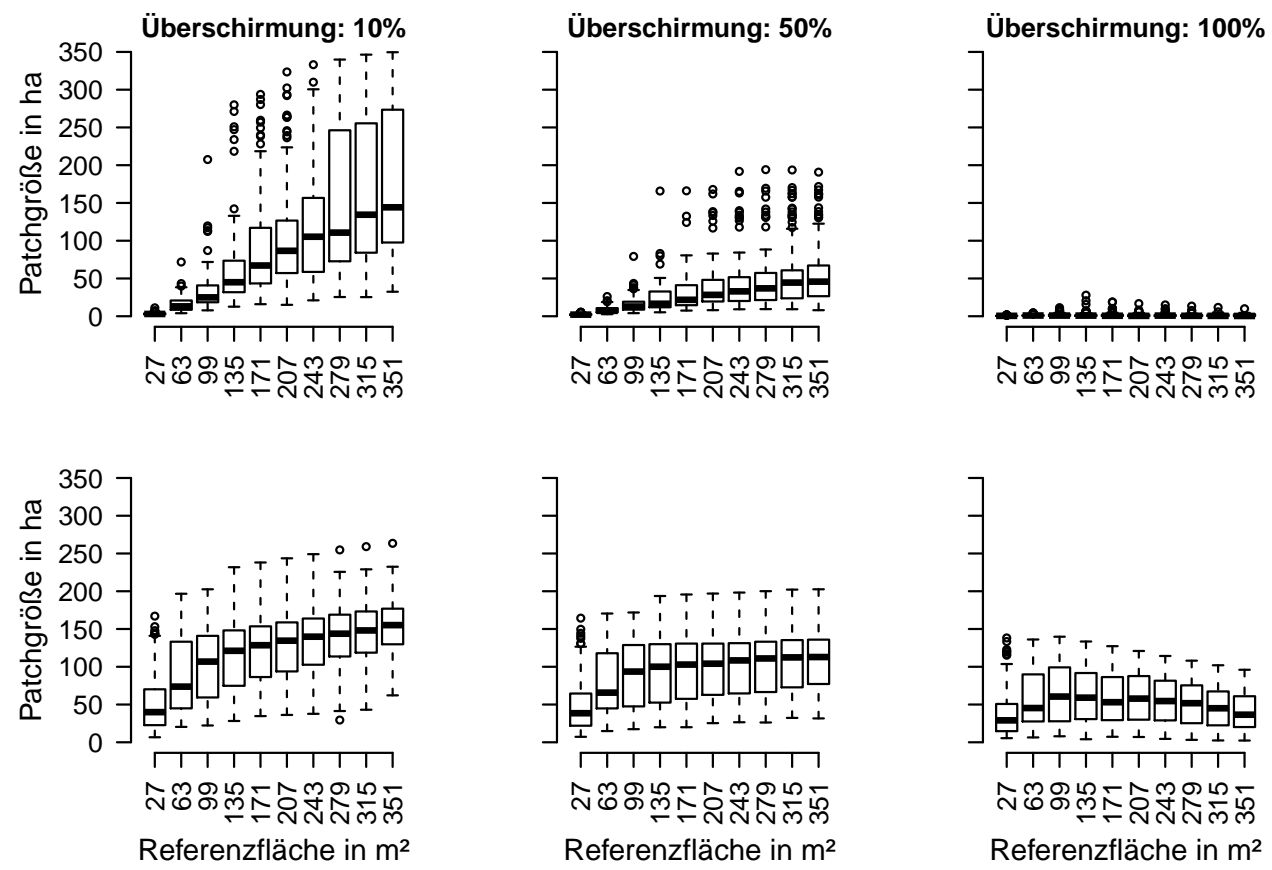

Abbildung 8.: Box-Whisker Plots der mittleren Patchgröße für verschiedene Referenzflächen und Mindestüberschirmungen dargestellt für $n=100$ stark fragmentierte ( $\alpha=0.7$, obere Reihe) und gering fragmentierte Landschaften $(\alpha=1.4$, untere Reihe) mit einem Kronenanteil von $30 \%$.

Referenzflächengröße. Wie anhand der geometrischen Analyse beschrieben, beruhen die Änderungen der Waldflächen hauptsächlich auf Verschiebungen der Waldränder. Dabei wird der Waldrand parallel zum Kronentrauf verschoben, so dass die Form des Waldrandes mehr oder weniger erhalten bleibt. Im Gegensatz dazu nimmt die mittlere Waldrandlänge für zunehmende Größen der Referenzflächen zu. Das ist jedoch nicht auf eine Änderung der Form, sondern im Wesentlichen auf die Zunahme der mittleren Patchgröße bzw. die Abnahme der Patchanzahl zurückzuführen. Es ist somit festzustellen, dass die Patchform durch Variation der Referenzfläche bzw. der Kronenüberschirmung nur geringfügig beeinflusst wird.

\subsubsection{Einfluss des Landschaftsmodells}

Durch das Versuchsdesign wurden Wechselwirkungen zwischen der Topographie und der Walddefinition ausgeschlossen, da zunächst die Waldkarten erstellt wurden und erst anschließend die Landschaftsanalyse mit unterschiedlichen Landschaftsmodellen erfolgte. Da- 

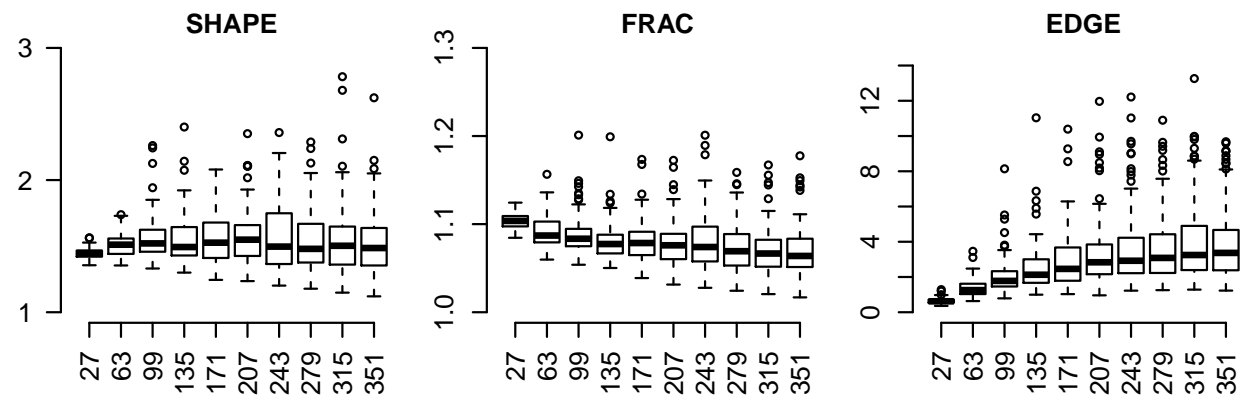

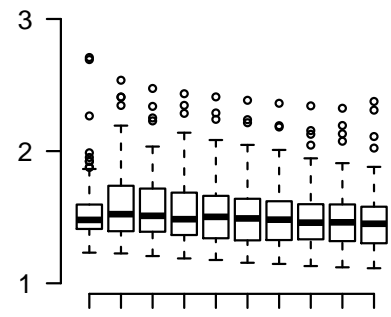

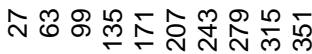

Referenzfläche

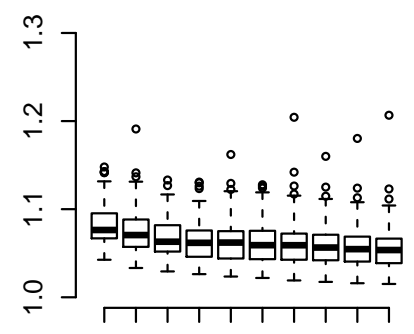

ลิ

Referenzfläche

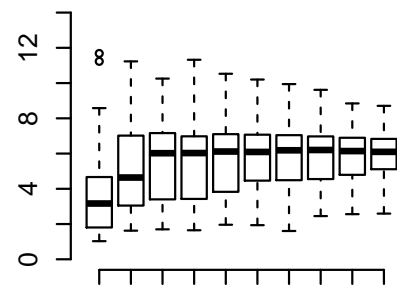

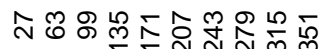

Referenzfläche

Abbildung 9.: Box-Whisker Plots der Landschaftsstrukturmaße für verschiedene Referenzflächen und einer Mindestüberschirmung $t=0.5$ dargestellt für $n=100$ stark fragmentierte ( $\alpha=0.7$, obere Reihe) und gering fragmentierte Landschaften ( $\alpha=1.4$, untere Reihe) mit einem Kronenanteil von $30 \%$.

her wird die weitere Auswertung der Ergebnisse auf eine Walddefinition mit einer Referenzfläche von $27 \mathrm{~m}^{2}$ und eine Mindestüberschirmung von $t=0.5$ beschränkt.

Tabelle 4.: Absolute Differenzen der zwei- und dreidimensionalen Landschaftsmodelle für Waldkarten mit einer Mindestüberschirmung von 0.5 und einer Referenzfläche von $27 p x$.

\begin{tabular}{rrrrr}
\hline Grundtyp & Waldanteil & Patchzahl & Patchgröße & Waldrandlänge \\
\hline$\alpha=1.4 ; p=0.3$ & 0.00 & 0.00 & 110.32 & 11.87 \\
$\alpha=1.4 ; p=0.7$ & 0.00 & 0.00 & 382.03 & 12.53 \\
$\alpha=0.7 ; p=0.3$ & 0.00 & 0.00 & 5.31 & 45.08 \\
$\alpha=0.7 ; p=0.7$ & 0.00 & 0.00 & 25.93 & 41.48 \\
\hline
\end{tabular}

Durch die Verwendung eines dreidimensionalen Landschaftsmodells kommt es in allen Fällen zu einer Vergrößerung der Gesamtfläche der untersuchten Landschaften, unabhängig von der Konfiguration und Komposition der gewählten Kronenkarten. Im Mittel verdreifacht sich die Waldfläche bei der Verwendung eines dreidimensionalen Landschaftsmodells. 
Die Vergrößerung hängt dabei direkt vom $\alpha$-Wert des simulierten Geländemodells ab, der die Oberflächenrauhigkeit beschreibt. In Tabelle 4 sind die Differenzen der zwei- und dreidimensionalen Landschaftsmodelle für einige Kenngrößen zusammengefasst. Dabei fällt auf, dass sich der Waldanteil nicht ändert. Dies lässt sich dadurch erklären, dass für die Simulation unabhängige, isotropische Zufallsfelder zur Modellierung der Landschaften und der Geländemodelle verwendet wurden. Daher vergrößern sich Wald- und Nicht-Waldflächen proportional, so dass der Waldanteil gleich bleibt. Die Patchanzahl ist aufgrund des Versuchsdesign für beide Landschaftsmodelle identisch. Ihre Form unterscheidet sich jedoch. In Abbildung 10 ist die Verteilung der SHAPE-, FRAC- und EDGE-Mittelwerte für beide Landschaftsmodelle gegenüber gestellt. Die größten Differenzen lassen sich für die $S H A$ $P E$-Werte bei stark fragmentierten Landschaften beobachten. Hier ist zu sehen, dass die Werte der dreidimensionalen Metriken höher liegen, also eine komplexere Form annehmen. Ein ähnlicher Trend zeigt sich für die fraktale Dimension und die mittlere Waldrandlänge, jedoch sind die Unterschiede wesentlich geringer.
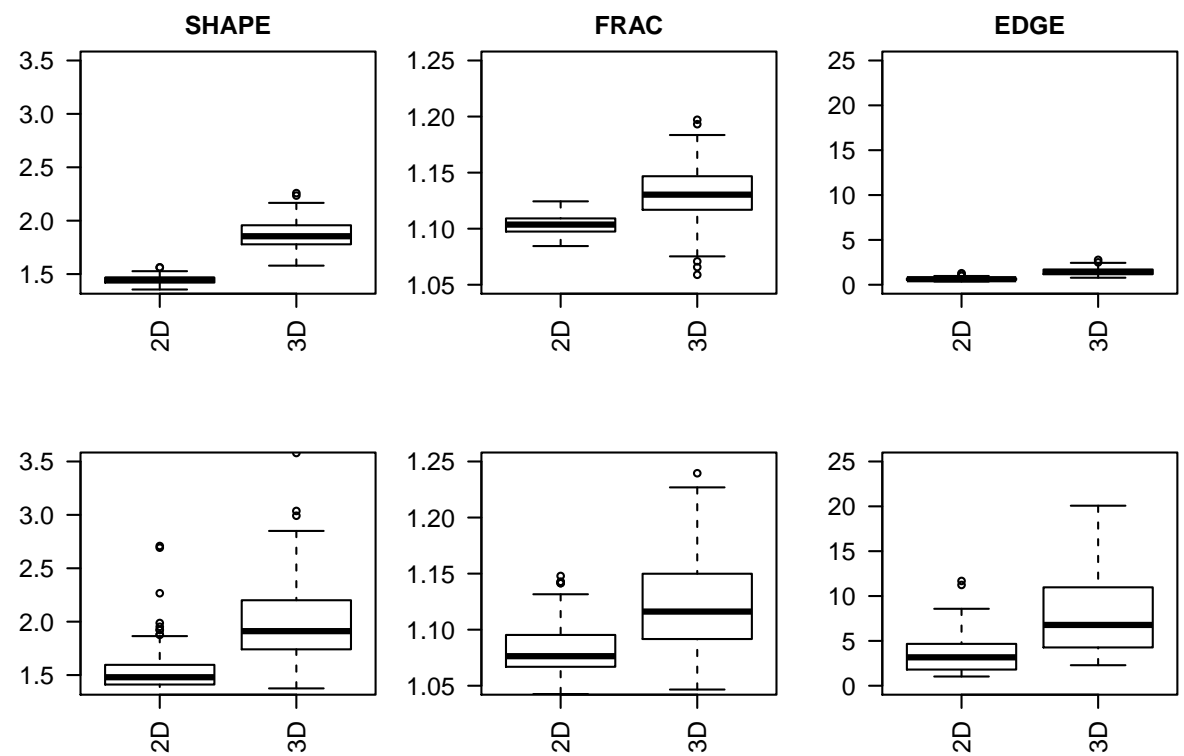

Abbildung 10.: Gegenüberstellung der zwei- und dreidimensionalen Landschaftsstrukturmaße für $n=100$ stark fragmentierte ( $\alpha=0.7$, obere Reihe) und gering fragmentierte Landschaften ( $\alpha=1.4$, untere Reihe) mit einem Kronenanteil von $30 \%$. 


\subsubsection{Skalen-Effekte}

Als vierter kritischer Faktor wurde der Beobachtungsmaßstab untersucht. Dazu wurden die zwei Aspekte horizontale und vertikale Auflösung getrennt in den Landschaftsmodellen variiert. Die Variation der horizontalen Aufösung erfolgt durch Aggregierung der Kronenkarten. Dabei wurden jeweils $3 \times 3 p x$ zusammengefasst. Durch die Variation der horizontalen Auflösung auf Ebene der Kronenkarten wurde im Gegensatz zu anderen Studien eine Analyse der Auflösungseffekte bei Anwendung des Referenzflächenverfahrens analysiert. Die Aggregierung wirkt damit nicht direkt auf die Waldfläche, in welcher Waldpixel aggregiert werden, sondern indirekt über die aggregierten Kronenpixel in der Referenzfläche. Daher ist eine Wechselwirkung mit der Wald-/Waldranddefinition zu prüfen.

Wie in Abbbildung 11 anhand eines Landschaftsausschnitts exemplarisch dargestellt, können im wesentlichen drei Effekte der horizontalen Auflösung beschrieben werden:

- es verschwinden einzelne kleine Waldflächen

- durch Teilung großer Flächen entstehen neue kleine Waldflächen

- es entstehen Lücken in Waldflächen, welche die Patchformen zum Teil erheblich ändern
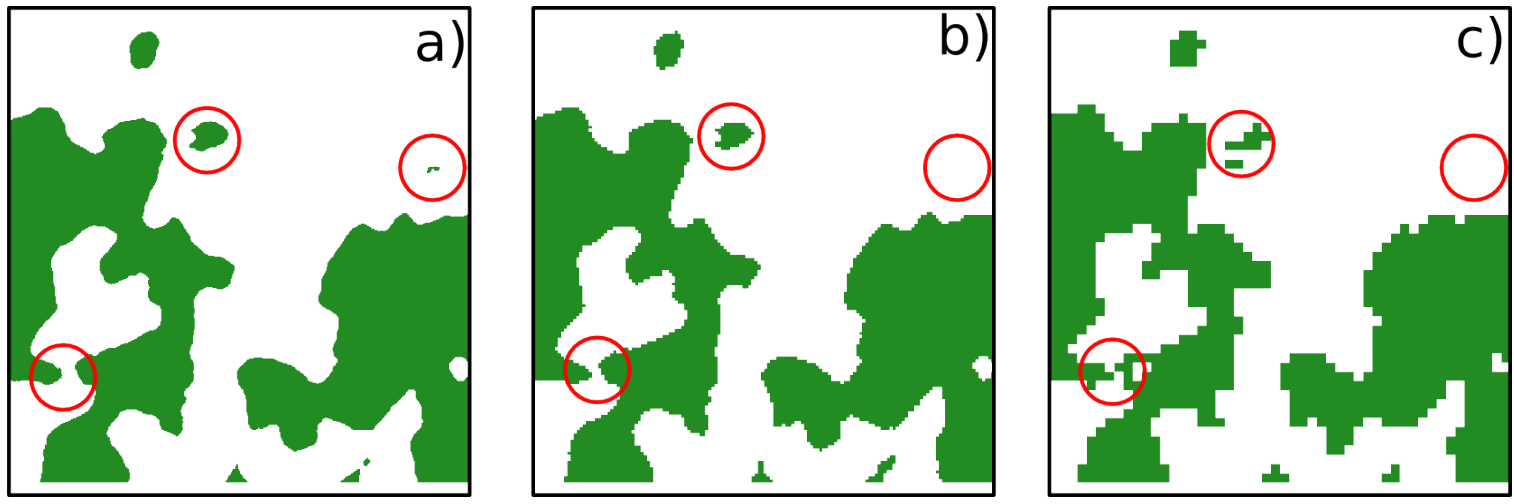

Abbildung 11.: Landschaftsausschnitt in drei verschiedenen horizontalen Auflösungen: a) $1 \mathrm{~m}$ b) $3 \mathrm{~m}$ und c) $9 \mathrm{~m}$. Die roten Kreise markieren drei Ausschnitte mit deutlichen Veränderungen für die verschiedenen Auflösungsstufen.

In Tabelle 5 sind die Differenzen zwischen zwei horizontalen Auflösungen über alle Landschaften zusammengefasst. Für die niedrigere Aufösung $(9 \mathrm{~m})$ sind geringere Patchanzahlen und Gesamtwaldrandlängen zu beobachten, während die mittlere Patchgröße steigt. Offensichtlich überwiegt somit das Verschwinden der Patches gegenüber dem in Abbildung 11 dargestellten Effekt, dass sich Waldflächen aufteilen. Bemerkenswert ist, dass der Waldanteil 
für alle vier Grundtypen konstant bleibt. Die hier untersuchten horizontalen Auflösungen beeinflussen den Waldanteil der Karten offensichtlich in viel geringerem Maß als z. B. die Walddefinition.

Tabelle 5.: Absolute Differenzen der Waldkarten mit horizontalen Aufösungen von 1m und $9 \mathrm{~m}$ mit einer Mindestüberschirmung von 0.5 und einer Referenzfläche von $27 \mathrm{~m}^{2}$.

\begin{tabular}{rrrrr}
\hline Grundtyp & Waldanteil & Patchzahl & Patchgröße & Waldrandlänge \\
\hline$\alpha=1.4 ; p=0.3$ & 0.00 & -0.26 & 2.28 & -0.05 \\
$\alpha=1.4 ; p=0.7$ & 0.00 & -0.06 & 5.44 & -0.03 \\
$\alpha=0.7 ; p=0.3$ & 0.00 & -6.82 & 0.33 & -0.70 \\
$\alpha=0.7 ; p=0.7$ & 0.00 & -5.11 & 2.24 & -0.59 \\
\hline
\end{tabular}

Neben der Anzahl und Größe der Patches kann sich auch eine Form der Patches ändern, wenn die horizontale Auflösung variiert wird (siehe Abbildung 11). Dabei verhalten sich die Form-Metriken unterschiedlich. Abbildung 12 zeigt nur für FRAC in stark fragmentierten Landschaften deutliche Differenzen zwischen beiden horizontalen Auflösungen. Die LSM $S H A P E$ und EDGE reagieren kaum auf die Variation der horizontalen Auflösung, was sich in den fast identischen Verteilungen zeigt.

Neben der horizontalen wurde auch die vertikale Auflösung der Geländemodelle in zwei Faktorstufen $(1 \mathrm{~m}, 25 \mathrm{~m})$ variiert. Abbildung 13 stellt die LSM der beiden vertikalen Auflösungsstufen einander gegenüber. Für die dargestellten LSM zeigen sich keine deutlichen Unterschiede der Mittelwerte zwischen beiden Auflösungsstufen. Es kann jedoch eine geringere Streuung in den Waldkarten mit $25 \mathrm{~m}$ vertikaler Aufösung beobachtet werden. Die größere Auflösung führt hier zu einer Vereinheitlichung der Patchformen. 

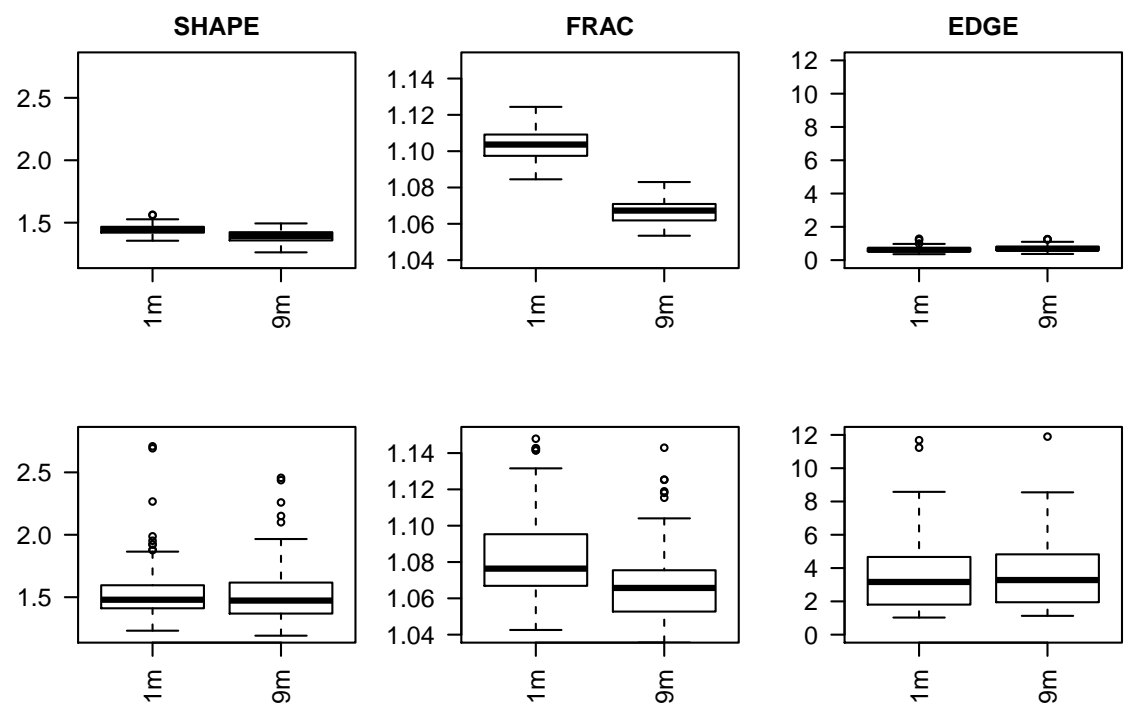

Abbildung 12.: Gegenüberstellung der Landschaftsstrukturmaße zweier horizontaler Auflösungen für $n=100$ stark fragmentierte ( $\alpha=0.7$, obere Reihe) und gering fragmentierte Landschaften ( $\alpha=1.4$, untere Reihe).
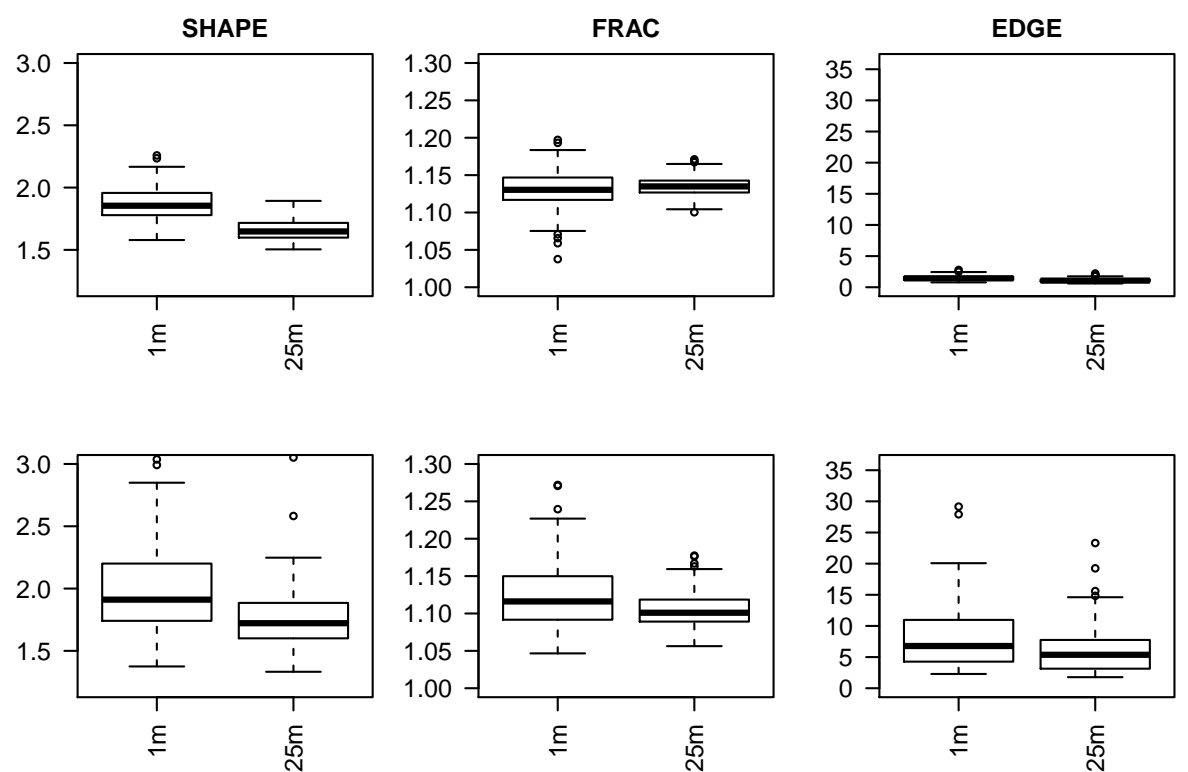

Abbildung 13.: Gegenüberstellung der Landschaftsstrukturmaße zweier vertikaler Auflösungen für $n=100$ stark fragmentierte ( $\alpha=0.7$, obere Reihe) und für gering fragmentierte Landschaften ( $\alpha=1.4$, untere Reihe). 



\section{Diskussion der Simulationsergebnisse}

Im Rahmen nationaler und internationaler Umweltabkommen werden zunehmend vielfältige Informationen über den Zustand, die Ausdehnung und Veränderung von Wäldern nachgefragt. Die Implementierung von Handelsmechanismen setzt dabei im Idealfall voraus, dass diese Informationen klaren Standards genügen, was vor allem impliziert, dass sie statistisch belastbar und nach objektiven Kriterien erfasst werden. Dafür müssen operational anwendbare Methoden zur Erfassung der Waldflächen als primäre Größe sämtlicher Waldinformationssysteme entwickelt werden. Die Simulation untersuchte vier in diesem Zusammenhang als kritisch eingestufte Faktoren und zeigte, dass sie einen wesentlichen Einfluss auf die Erfassung der Waldfläche haben können. Die Faktoren wirken sehr unterschiedlich auf den Waldanteil, die Waldflächenstruktur und die Form der Waldflächen.

Über Walddefinitionen wird seit Beginn der gezielten Bewirtschaftung von Wäldern und der systematischen Erfassung der forstlichen Ressourcen diskutiert (vergl. Abschnitt 3.1 und 3.2). Durch die veränderten gesellschaftlichen Ansprüche an die Wälder werden von verschiedenen Disziplinen bzw. Sektoren immer wieder neue Kriterien genutzt, um Wald gegenüber anderen Landnutzungen abzugrenzen, so dass die Anzahl möglicher Elemente einer Walddefinition kontinuierlich steigt. In allen Fällen muss eine Grenzlinie (Waldrand) zwischen Wald- und Nicht-Waldflächen gezogen werden, um Waldflächen auszuweisen. Die Lage dieser Grenzlinie muss durch die Walddefinitionen explizit festgelegt werden. In der vorliegenden Simulation wurden die Effekte zweier möglicher Elemente der Walddefinition, Kronenüberschirmung und die Referenzfläche, auf welcher die Überschirmung gemessen wird, analysiert.

Als Schnittstelle zur Atmosphäre beeinflusst das Kronendach maßgeblich Kohlenstoffkreislauf, Mikroklima und Produktivität eines Waldes (Ozanne et al., 2003). Die Kronenüberschirmung quantifiziert diese Schnittstelle und stellt somit im Prinzip ein geeignetes Kriterium zur Abgrenzung von Wald dar, was sich in der häufigen Verwendung in internationalen Walddefinitionen widerspiegelt. Ein zusätzlicher Grund für die häufige Verwendung dieses Kriteriums ist die weit verbreitete Annahme, dass sich die Kronüberschirmung sowohl fernerkundlich als auch terrestrisch „einfach“ messen lässt. Da die Kronenüberschirmung ein Verhältnis angibt, muss eine Referenzfläche als Bezugsgröße festgelegt werden. Erst gemeinsam legen Kronenüberschirmung und Referenzfläche die Lage des Waldrandes 
explizit fest und ermöglichen die Ausweisung von Waldflächen. Der Einsatz von Fernerkundungsdaten gewinnt im Rahmen von Waldinventuren an Bedeutung. Dies ist hauptsächlich auf die technologische Entwicklung zurückzuführen, die zu einer verbesserten Bildqualität und zur Senkung der Aufnahmekosten führt. Ein weiterer Grund ist in den Kriterien der Walddefinitionen selbst zu sehen. So gibt es bisher keine praktikablen terrestrischen Stichprobenverfahren zur Erfassung der Kriterien „Mindestgröße“ und „Mindestbreite“ . Der Einsatz von Fernerkundungsdaten erscheint daher besonders dann sinnvoll, wenn eine Kombination mit terrestrischen Messungen erfolgt. Dies setzt jedoch voraus, dass die Definitionen für beide Verfahren kompatibel sind. Die Referenzfläche bestimmt die Größe der Beobachtungsfläche für das Kriterium Kronenüberschirmung. In Fernerkundungsdaten kann nahezu jede beliebige Form und Größe der Referenzfläche abgebildet werden. Für terrestrische Erhebungen ergeben sich praktische Limitationen, da die Größe der Referenzfläche die Mindestgröße der Stichprobenfläche, auf der die Überschirmung im Feld gemessen wird, festlegt. Als Ausgangspunkt für die Diskussion über die geeignete Referenzflächengröße können daher Untersuchungen zur optimalen Plotgröße bei Waldinventuren, wie sie unter anderem bei Zeide (1980) beschrieben sind, dienen. In der praktischen Anwendung des Kriteriums Kronenüberschirmung können in der Literatur vier Ansätze zur Definition der Referenzflächen identifiziert werden:

1. Es wird eine Mindestüberschirmung festgelegt, ohne eine Referenzfläche explizit zu definieren. Dieser Ansatz wird häufig im Zusammenhang mit fernerkundlichen Waldflächenkartierungen auf Basis von Bildern mit geringer räumlicher Auflösung verwendet. Hier wird die Kronenüberschirmung aus den Spektralinformationen eines Pixels modelliert, so dass die Pixelgröße letztendlich die Referenzfläche bestimmt. Dieser Ansatz führt zu erheblichen Definitionslücken, wie die Simulationsergebnisse zeigen.

2. Der Waldrand/die Waldbegrenzungslinie (WBL) wird über eine maximal zulässige Distanz zwischen zwei Bäumen definiert. Dieses Abstandsverfahren wird im Schweizer Landesforstinventar angewendet (Zingg \& Bachofen, 1988). Die Referenzfläche wird hierbei flexibel durch den maximalen Abstand zwischen Bäumen definiert, womit sich für jeden Untersuchungspunkt unterschiedliche Formen und Größen der Referenzflächen ergeben. Lanz (2005) weist darauf hin, dass dieses Verfahren zwar geeignet ist, um eine Wald-/Nicht-Waldentscheidung für den Stichprobenpunkt zu fällen, nicht jedoch um den Verlauf des Waldrandes eindeutig räumlich festzulegen. Zingg \& Bachofen (1988) zeigen, dass es durch die Variation der maximalen Distanz zu einer Verschiebung der WBL und damit verbunden einer Änderung der Referenzfläche kommt. 
3. Die Referenzfläche wird durch Triangulation der Baumpositionen bestimmt. Dabei wird jeweils für drei benachbarte Bäume eine Referenzfläche festgelegt. Der Waldrand verläuft dann entlang der äußeren Triangulationslinien. Das Triangulationverfahren wurde von Eysn et al. (2012) vorgestellt. Es bietet den Vorteil, dass bei bekannten Baumpositionen, die z. B. aus LiDAR Punktwolken ermittelt werden, eine automatische Delinierung erfolgen kann. Da keine fixe Referenzfläche festgelegt wird, ist der Verlauf des Waldrandes ausschließlich von der Verteilung der Baumpositionen abhängig. Dies hat den Vorteil, dass keine Referenzfläche definiert werden muss. Problematisch ist jedoch, dass sich das Verfahren in terrestrischen Inventuren kaum umsetzen lässt. Außerdem stellt es sehr hohe Anforderungen an die Auflösung und Auswertung von Fernerkundungsdaten, da alle Baumpositionen bekannt sein müssen, um die Referenzflächen zu bestimmen.

4. Das in der vorliegenden Arbeit verwendete Referenzflächenverfahren bezieht sich auf eine fixe Referenzfläche. Das hat den Vorteil, dass die Lage des Waldrandes eindeutig festgelegt ist. Außerdem stellt die Verwendung von fixen Referenzflächen die Kompatibilität zu terrestrischen Inventuren sicher, da sie es ermöglicht, sowohl bei der terrestrischen Inventur als auch bei der fernerkundlichen Auswertung die selben Referenzflächen zu verwenden. Für die fernerkundliche Waldflächenerfassung ergibt sich des Weiteren der Vorteil, dass nicht alle Bäume einzeln erfasst werden müssen, wodurch deutlich niedrigere Kosten für die Daten und Auswertung angesetzt werden können. Den Pixeln müssen lediglich die Klassen Baumkrone/Nichtbaumkrone zugeordnet werden. Aus diesen Kronenkarten können dann mit Moving-Window-Operatoren direkt Waldkarten abgeleitet werden. Neben den genannten Vorteilen des Referenzflächenverfahrens ergeben sich auch einige Nachteile. So ist der Verlauf des Waldrandes in vielen Fällen im Feld nicht einfach zu bestimmen, da er entlang des Überschirmungsgradienten verläuft. Dies ist verstärkt bei Überschirmungen der Fall, die von $50 \%$ verschieden sind, da hier der Waldrand teilweise im Bestand oder außerhalb des Bestandes liegt. Außerdem führt die Verwendung von Moving-Window-Operatoren dazu, dass am Bildrand ein Teil der Karte abgeschnitten werden muss.

Die Ergebnisse der vorliegenden Simulationen zeigen, dass die Mindestüberschirmung und die Größe der Referenzfläche einen starken Einfluss sowohl auf den Waldanteil als auch auf die Struktur der Waldfläche haben können. Für fixe Referenzflächen ist ihre Wirkung durch eine starke Interaktion geprägt, welche einem speziellen Muster folgt, das bezogen auf die Waldfläche, schon von Kleinn $(1991,2001)$ beschrieben wurde. Mit Hilfe von geometrischen 
Simulationen des Waldrandes konnten diese Interaktionen in der vorliegenden Arbeit für geradlinig verlaufende Waldränder mit einem geometrischen Modell erklärt werden. Das Modell führt die beobachteten Veränderungen der Waldflächen auf die, durch Variation von Mindestüberschirmung und Referenzfläche bedingte, Verlagerung der Waldränder zurück. Dabei wirkt die Mindestüberschirmung hauptsächlich auf die Richtung und die Referenzfläche auf die Stärke der Verschiebung. Anhand der empirischen Analyse auf Grundlage der simulierten Landschaften konnte gezeigt werden, dass sich das Modell auch auf komplexere Waldlandschaften übertragen lässt. Letztendlich stellt die Referenzfläche somit eine Waldranddefinition dar, welche jedoch nur im Zusammenhang mit einer Mindestüberschirmung den exakten Verlauf des Waldrandes definiert.

Eine Einigung auf eine einheitliche Walddefinition im Rahmen der internationalen Umweltabkommen erscheint unwahrscheinlich, da in den Vertragsstaaten sehr unterschiedliche Waldtypen vorkommen, die nur schwer von einer globalen Walddefinition erfasst werden können. Viel wahrscheinlicher ist es, dass man sich auf eine Spannweite verschiedener Schwellenwerte einigt, wie es jetzt schon der Fall für die Kronenüberschirmung im Rahmen CDM Programmes ist. Hier werden Werte zwischen 10\% und 30\% akzeptiert (COP 7/Entscheidung 11). Die exakte Festlegung des Schwellenwertes für die Mindestüberschirmung ist in vielen Fällen eine politische Entscheidung. Die Größe der Referenzfläche fand in diesen Debatten bisher keine Berücksichtigung. Aus wissenschaftlicher Sicht können aber sowohl ökologische als auch praktische Argumente zur Eingrenzung geeigneter Schwellenwerte gefunden werden. Sasaki \& Putz (2009) fordern, dass zukünftig im Rahmen der UNFCCC Prozesse zwischen „dense forest" und „open forest “ unterschieden wird. Für letztere schlagen sie einen Schwellenwert von $40 \%$ vor. Sie begründen dies mit den ökologischen Konsequenzen welche mit einer Reduktion der Überschirmung von $100 \%$ auf z. B. 10\% einhergehen. Durch die Verwendung von Walddefinitionen mit einem sehr niedrigen Schwellenwert (z. B. 10\%) würden viele Waldfunktionen stark eingeschränkt werden ohne dass diese Veränderungen erfasst wären. Im Zusammenhang mit REDD grenzt die Mindestüberschirmung die Prozesse Entwaldung und Degradation voneinander ab und hat damit weitreichende Konsequenzen für den Erfolg des Programms (Putz \& Redford, 2010; Sasaki \& Putz, 2009). Die Ergebnisse der vorliegenden Simulation geben einen weiteren Hinweis, dass höhere Schwellenwerte für eine funktionale und operationale Walddefinition besser geeignet sind. Hier konnte gezeigt werden, dass ein Schwellenwert von 50\% die Effekte der Referenzfläche minimiert. Unter der Annahme, dass die Vertragstaaten unterschiedliche Schwellenwerte festlegen werden, sind Vergleiche der Waldflächen nur dann möglich, wenn die Unterschiede rechnerisch ausgegli- 
chen werden können. Die COST E43 Initiative strebt die Etablierung von Modellen (bridges) zum Ausgleich der definitionsbedingten Differenzen auf europäischer Ebene an (Vidal et al., 2008). Die Ergebnisse der Simulation zeigen, dass solche „bridges“ in Form linearer Modelle nur für Gebiete mit wenig fragmentierten Wäldern sinnvoll angepasst werden können.

Die UNCBD erfordert ein Waldflächenmonitoring, welches im Gegensatz zum UNFCCC, wo lediglich die absolute Änderung der Waldflächen (activity data) erfasst werden, auch die räumlichen Landschaftsmuster erfasst. Die Ergebnisse zeigen, dass die Erfassung der Waldfragmentierung mit LSM prinzipiell möglich ist aber wesentlich von der Walddefinition beeinflusst wird. In ihrer Reaktion auf die Änderungen der Walddefinition unterscheiden sie sich. LSM, welche die Anzahl und Größe der Patches beschreiben, reagieren sensitiv auf die Änderungen der Wald- und Waldranddefinition. LSM, welche die Form der Patches beschreiben zeigen kaum Unterschiede zwischen für den verschiedenen Wald- und Waldranddefinitionen. Ein ähnliches Verhalten der LSM beschrieb Colson et al. (2009). Sie fassten unterschiedliche Landbedeckungsklassen zusammen um den Einfluss der thematischen Walddefinition auf verschiedene LSM zu untersuchen. Für den brasilianischen Bundesstaat Rondônia konnten sie zeigen, dass die Formmetriken gegenüber den verschiedenen Walddefinitionen kaum sensitiv reagierten. Die Konfigurationsmetriken, z. B. Patchanzahl, schwanken jedoch stark. Bei der Entwicklung eines Biodiversitätsmonitorings muss daher besonders bei der Verwendung von Konfigurationsmetriken auf eine einheitliche Walddefinition geachtet werden.

Die Berechnung von LSM für dreidimensionale Landschaften ist ein relativ neuer Ansatz. Das hier gewählte Verfahren zur Berechnung der korrigierten Flächen und Umfänge nach Jenness (2004) hat sich dabei als praktikabel und zielführend erwiesen und konnte aufgrund der neu entwickelten Software patch $3 D$ auch effizient auf große Landschaftsmodelle angewendet werden. Stupariu et al. (2010) vergleichen dieses Verfahren mit zwei anderen Triangulationsverfahren hinsichtlich ihrer Differenzen bei der topographischen Korrektur von Patchflächen und Umfängen und stellten nur geringe Unterschiede fest. Sie schlussfolgerten, dass prinzipiell alle drei Triangulationsverfahren geeignet sind. Im Rahmen der Simulation wurde eine isotrope Waldverteilung unterstellt. Das heißt, dass zwischen der Topographie und der Waldverteilung keine Abhängigkeiten bestehen. Dies muss für „echte" Landschaften bezweifelt werden. Häufig ist die Bewirtschaftung der Landschaft durch das Relief geprägt. Insofern ist die Übertragbarkeit der Ergebnisse auf reale Landschaft nur eingeschränkt möglich. Die Ergebnisse zeigten, dass die Formmetriken SHAPE und FRAC sensitiv auf Unterschiede im Landschaftsmodell reagieren. Dies stimmt mit den Ergebnissen 
von Hoechstetter et al. (2008) überein, die zeigten das die FRAC und SHAPE Werte für eine zunehmende Oberflächenrauhigkeit der Landschaften zunehmen. Die vorliegenden Ergebnisse der dreidimensionalen Landschaftsanalyse sind konsistent zu den Ergebnissen anderer Studien. Daher kann geschlussfolgert werden, dass die verwendeten Methoden grundsätzlich geeignet sind, dreidimensionale Landschaftsstrukturen zu beschreiben. Ob sich daraus Vorteile im Rahmen eines Biodiverstiätsmonitorings ergeben, muss jedoch in weiteren Untersuchungen geklärt werden. Dazu müsste geprüft werden, inwiefern die Berücksichtigung der topographischen Muster eine verbesserte Beschreibung der verschiedenen ökologischen Prozesse (z. B. Migration, Isolation, Extinktion) erlaubt.

Das REDD-Programm zielt auf den Erhalt und die Verbesserung der Kohlenstoffspeicherfunktionen von Landschaften ab. Zur Ermittlung der Kohlenstoffvorräte werden die Kohlenstoffdichten (emission factors) einer bestimmten Landbedeckungsklasse mit den dazugehörigen Flächen (activity data) multipliziert. Daraus ergibt sich der Bedarf, die Landbedeckungsklassen, z. B. Wald, einheitlich zu definieren. Aus naturwissenschaftlicher Sicht ist zur Erfassung der Kohlenstoffvorräte eine Einteilung der Landschaft in diskrete Klassen jedoch nicht zwingend notwendig. Bezogen auf das eigentliche Anliegen der Klimaschutzprogramme, die Emissionsreduktion, hätte eine Erfassung der Kohlenstoffvorräte und deren Änderung auf Landschaftsebene ohne Einteilung in Landbedeckungsklassen, wie sie auch von Putz \& Sasaki (2009) vorgeschlagen wird, Vorteile. Zum Beispiel würden in einem solchen Verfahren neben den im Wald befindlichen Bäumen auch solche außerhalb des Waldes erfasst werden. Kleinn et al. (2005) zeigten im Rahmen einer Pilotstudie zur nationalen Waldinventur für Costa Rica, dass $8.2 \%$ des Baumholzvolumens außerhalb des Waldes zu finden sind. Diese Ressourcen haben neben ihrer Funktion als Kohlenstoffspeicher und ihrem kommerziellen Wert auch große ökologische Bedeutung. Daher finden sich auch in der Landschaftsökologie vermehrt Ansätze ökologische Gradienten zu beschreiben, statt das diskrete PCM-Modell anzuwenden. Da die Simulation zeigte, dass die meisten LSM sensitiv auf Veränderungen der Walddefinitionen reagieren, würden auch für die Beschreibung der Waldfragmentierung kontinuierliche Ansätze von Vorteil sein. Konzepte zur Erfassung der Landschaftsstruktur basierend auf Gradienten wurden unter anderem von McGarigal \& Cushman (2005) vorgestellt. Im Rahmen dieser Arbeit wurde eine Methode zur Waldflächenkartierung entwickelt, die sowohl binäre Waldkarten zur diskreten als auch Kronendichtekarten zur kontinuierlichen Auswertung zur Verfügung stellt. Die Kronendichtekarten könnten als Grundlage für die kontinuierliche Erfassung der holzigen Biomasse und darin enthaltenen Kohlenstoffvorräte auf Landschaftsebene verwendet werden. Außerdem 
eignen sie sich als Grundlage für das Gradienten-Verfahren zur Beschreibung der Landschaftsstruktur.

Ein weiterer Vorteil der kontinuierlichen Schätzung der holzigen Biomasse ist die Möglichkeit, Veränderungen innerhalb von Klassen zu erfassen. Entwaldung kann in Form von binären Variablen beschrieben werden (Wald/Nicht-Wald). Aber Degradierung folgt einem Kontinuum und muss daher durch kontinuierliche Variablen erfasst werden (Putz \& Redford, 2010). Die Degradierung von Wäldern ist wichtiger Bestandteil des REDD-Programms und namentlich in Form des zweiten D's verankert. Die Kronendichtekarten sind daher prinzipiell geeignet zur Erfassung der Degradierung von Wälder beizutragen. Mathys et al. (2006) stellen die Vorteile eines kontinuierlichen Ansatzes bei der Waldkartierung dar. Sie weisen daraufhin, dass verschiedene Nutzungskonzepte unterschiedliche Walddefinitionen benötigen. So kann man leicht erkennen, dass die Walddefinition für die Erfassung der Kohlenstoffvorräte eine andere sein wird als zur Beschreibung der Biodiversität. Daraus kann geschlussfolgert werden, dass ein Ansatz, der das gleichzeitige Abbilden verschiedener Walddefinitionen basierend auf einheitlichen Kriterien erlaubt, eine wesentliche Verbesserung der Waldressourcenerfassung darstellt.

Aus Sicht der politischen und praktischen Implementierung von internationalen Umweltabkommen (z. B. UNFCCC) wird eine Einteilung in Landbedeckungsklassen weiterhin wichtig sein, da kontinuierliche Ansätze schwer zu kommunizieren sind und ein fächerübergreifender Ansatz erfordern. Zur Zeit sind die Zuständigkeiten von Verhandlungsteilnehmern und Behörden meist an bestimmte Landnutzungen gebunden, was die politische Implementierung von kontinuierlichen Ansätzen verkompliziert. Das die Verwendung weniger diskreter Klassen in der Geographie üblich ist, erschwert den Einsatz von kontinuierliche Verfahren zusätzlich. 



\section{Schlussfolgerungen für die Standardisierung der Waldflächenerfassung}

Vier Schlussfolgerungen für die Entwicklung eines standardisierten Verfahrens zur fernerkundlichen Erfassung der Waldfläche ergeben sich aus der Simulation:

- Die Walddefinition hat signifikanten Einfluss auf die Waldfläche. Unterschiede in der Walddefinition führen zu einer Änderung der Waldanteile und der räumlichen Anordnung der Waldflächen. Um vergleichbare und konsistente Waldkarten zu erhalten, muss eine einheitliche Walddefinition verwendet werden.

- Neben der Mindestüberschirmung hat die Referenzfläche einen großen Einfluss auf die Waldflächen und deren Fragmentierung. Um eine Kombination mit terrestrischen Inventuren zu erleichtern, sollte die Referenzflächengröße so gewählt werden, dass sie den Stichprobenflächen einer Waldinventur entspricht.

- Das Referenzflächenverfahren kann mit Hilfe von Moving-Window Operatoren zur Bestimmung der Kronenüberschirmung in Bilddaten eingesetzt werden. Dies ermöglicht die Implementierung einer spezifischen Waldranddefinition.

- Für die Anwendung des Referenzflächenverfahrens ist die Unterscheidung von Kronenund Nicht-Kronenpixeln erforderlich. Dies ist per Definition nur mit räumlich hoch auflösenden Bildern möglich. Die Simulation zeigte jedoch, dass die räumliche Auflösung nur einen geringen Einfluss auf die Waldflächenschätzung hat. Daher ist es für die Waldflächenschätzung ausreichend, Bilder mit einer räumlichen Auflösung zu verwenden, die eine Unterscheidung zwischen Kronen und Nicht-Kronen „gerade noch“ zulässt. 



\section{Teil III.}

Entwicklung eines Verfahrens zur Standardisierung der fernerkundlichen Waldflächenerfassung 



\section{Integration einer spezifischen Walddefinition in die Auswertung von Fernerkundungsdaten}

Die Analyse der räumlichen Simulation im zweiten Teil der vorliegenden Arbeit zeigte, dass die Walddefinition einen erheblichen Einfluss auf die Größe und Form der Waldfläche hat. Daraus muss geschlussfolgert werden, dass es für die Erstellung von standardisierten, somit vergleichbaren, Waldflächenkarten erforderlich ist, vorher einheitlich festgelegte Walddefinitionen anzuwenden. Bisherige Studien entwickelten eine Vielzahl von Verfahren zur Klassifikation unterschiedlichster Bilder von verschiedenen Sensoren. Die Implementierung einer spezifischen Walddefinition im Rahmen der Klassifikation von Fernerkundungsdaten wurde bisher jedoch kaum untersucht. Für den erfolgreichen Einsatz von Fernerkundungsdaten zur Erfüllung der Berichtspflichten, die sich aus internationalen Umweltabkommen ergeben, ist die Implementierung einer Walddefinition, die kompatibel zu den Walddefinitionen der terrestrischen Aufnahmen ist, jedoch erforderlich.

Für die Entwicklung eines standardisierten Verfahrens zur Waldflächenerfassung soll die FAO-Walddefinition, wie sie im globalen Waldbericht (engl. Global Forest Resources Assessment, FRA) (FRA, 2010b) beschrieben ist, genutzt werden. Diese Definition wird von den meisten internationalen Umweltabkommen verwendet (vergl. Abschnitt 3.1). Die FAO definiert Wald als eine Landnutzungsform. Insgesamt werden vier verschiedene Landnutzungsklassen unterschieden:

i) Wald (engl. forest)

ii) andere mit Bäumen bestockte Flächen (engl. other wooded land)

iii) andere Flächen (engl. other land)

iv) andere Flächen mit Bäumen (engl. other land with tree cover)

Jede dieser Klassen wird wiederum durch eine Kombination von quantiativen und qualitativen Kriterien beschrieben. Da diese Kriterien für die Entwicklung des Klassifikationsschlüssels ausschlaggebend sind, sind sie in Tabelle 6 aufgeführt. 
Tabelle 6.: Landnutzungsklassen nach FAO-Waldbericht. Quelle: (FRA, 2010b).

\begin{tabular}{|c|c|c|}
\hline Name & Abkürzung & Beschreibung \\
\hline Forest & FOREST & $\begin{array}{l}\text { Lands spanning more than } 0.5 \text { ha with trees } \\
\text { higher than } 5 \mathrm{~m} \text { and canopy cover of more } \\
\text { than } 10 \% \text { or trees able to reach these thres- } \\
\text { holds in situ. It does not include land that } \\
\text { is predominantly under agricultural or urban } \\
\text { land use. }\end{array}$ \\
\hline Other wooded land & OWL & $\begin{array}{l}\text { Land not classified as "Forest", spanning mo- } \\
\text { re than } 0.5 \text { ha; with trees higher than } 5 \mathrm{~m} \text { and } \\
\text { a canopycover of } 5-10 \% \text {, or trees able to reach } \\
\text { these thresholds in situ; or with a combined } \\
\text { cover of shrubs, bushes and trees above } 10 \% \text {. } \\
\text { It does not include land that is predominant- } \\
\text { ly under agricultural or urban land use. }\end{array}$ \\
\hline Other land & $\mathrm{OL}$ & $\begin{array}{l}\text { All land that is not classified as "Forest"or } \\
\text { "Other wooded land" }\end{array}$ \\
\hline Other land with tree cover & OLTC & $\begin{array}{l}\text { Land classified as "Other land", spanning } \\
\text { more than } 0.5 \text { ha with a canopy cover of more } \\
\text { than } 10 \% \text { of trees able to reach a height of } \\
5 \mathrm{~m} \text { at maturity. }\end{array}$ \\
\hline Water & WATER & Waterbodies \\
\hline
\end{tabular}

Für die Landnutzungsklasse Wald werden drei quantitative Kriterien genannt und Grenzwerte festgelegt:

i) Mindestfläche (0.5ha)

ii) Mindesthöhe (5m)

iii) Mindestüberschirmung (10\%)

$\mathrm{Zu}$ beachten ist, dass es sich bei der Mindesthöhe und der Mindestüberschirmung um potentielle Grenzwerte handelt, welche die Bäume erst im ausgewachsenen Zustand in situ erreichen müssen (vergl. Tabelle 6). Dieser Zusatz wurde aufgenommen, um Wiederaufforstungsflächen oder Windwürfe zur Waldfläche zu zählen, auch wenn die Schwellenwerte temporär nicht erreicht sind. Neben den quantitativen Kriterien werden auch qualitative Kriterien in der Walddefinition genannt, welche ausschließen, dass die Flächen überwiegend landwirtschaftlich oder für Siedlungen genutzt werden. Um eine Wald/Nicht-Waldentscheidung zu treffen, müssen theoretisch alle genannten Kriterien gemessen werden. Praktisch ist diese 
jedoch kaum umsetzbar, da man die potentielle Höhe eines ausgewachsenen Baumes nicht an einem jungen messen kann. Im Folgenden wird daher davon ausgegangen, dass die Grenzwerte zum Aufnahmezeitpunkt erreicht sein müssen.

Die Klassifikation von Fernerkundungsdaten basiert auf Unterschieden in den spektralen Signaturen verschiedener Landbedeckungsklassen. Die FAO definiert jedoch Landnutzungsklassen (siehe Tabelle 6) basierend auf den genannten Kriterien (z. B. Kronenüberschirmung, Flächengröße, vorherrschende Landnutzung), welche sich spektral oft nicht eindeutig unterscheiden lassen. Um die FAO-Landnutzungsklassen ansprechen zu können, wurde ein hierarchisches Klassifkationsschema mit drei aufeinander aufbauenden Klassifikationschlüsseln entwickelt, wie es in Abbildung 14 dargestellt ist.

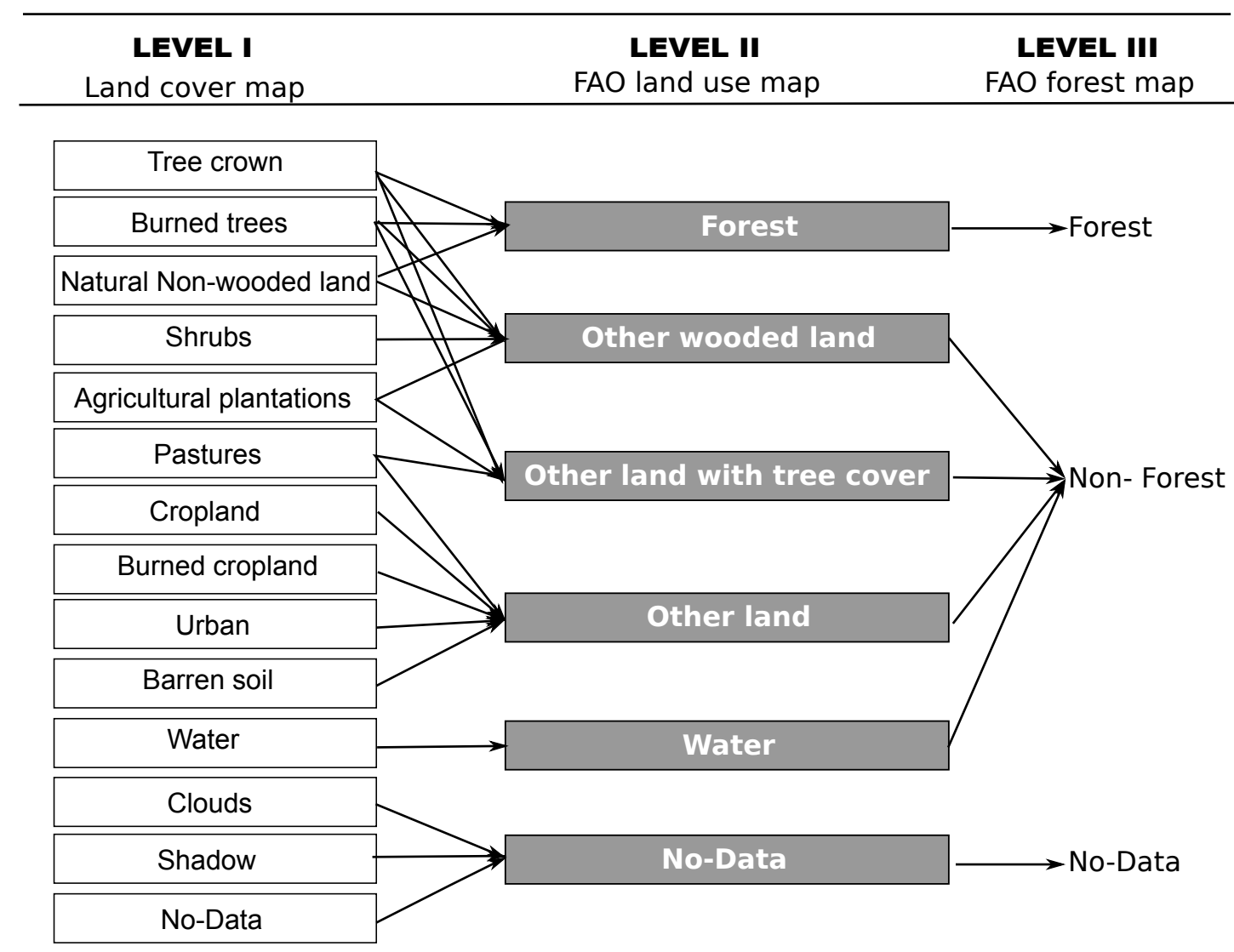

Abbildung 14.: Hierarchischer Klassifikationsschlüssel zur Ableitung der FAOLandnutzungsklassen. Quelle: Magdon et al. (2013).

Abbildung 14 zeigt drei Ebenen der Klassifikation mit den jeweiligen Klassifikationsschlüsseln. Auf der niedrigsten Ebene (Level-I) werden 14 Landbedeckungsklassen direkt im Bild 
unterschieden. Die Klassen sind dabei so gewählt, dass sie sich spektral gut differenzieren lassen und später in die von der FAO definierten Landnutzungklassen überführt werden können, welche sich in der zweiten Ebene (Level-II) befinden. Auf der obersten Ebene (Level-III) steht dann die eigentlich gewünschte Klassifikation mit den Klassen Wald und Nicht-Wald. Die Pfeile zwischen den drei Ebenen zeigen an, wie die einzelnen Klassen aggregiert werden. Dabei ist zu sehen, dass der Übergang zwischen der Ebene II und III eindeutig, und von I auf II nicht eindeutig ist. Das heißt, dass die Level-I Klassen nicht direkt den FAO-Klassen zugeordnet werden können, da hier die Definition der einzelnen Klassen zu berücksichtigen ist. Zur Implementierung der Kriterien der jeweiligen Landnutzungsklasse wurde der Entscheidungsbaum, der in Abbildung 15 dargestellt ist, entwickelt. Der Entscheidungsbaum basiert auf dem Referenzflächenverfahren (vergl. Abschnitt 3.2). Das heißt, dass die Kriterien vorherrschender Landnutzungstyp, Baumkronenüberschirmung und Strauchüberschirmung auf einer Referenzfläche mit einheitlicher Größe für jedes Pixel der Level-I Klassifikation bestimmt werden.

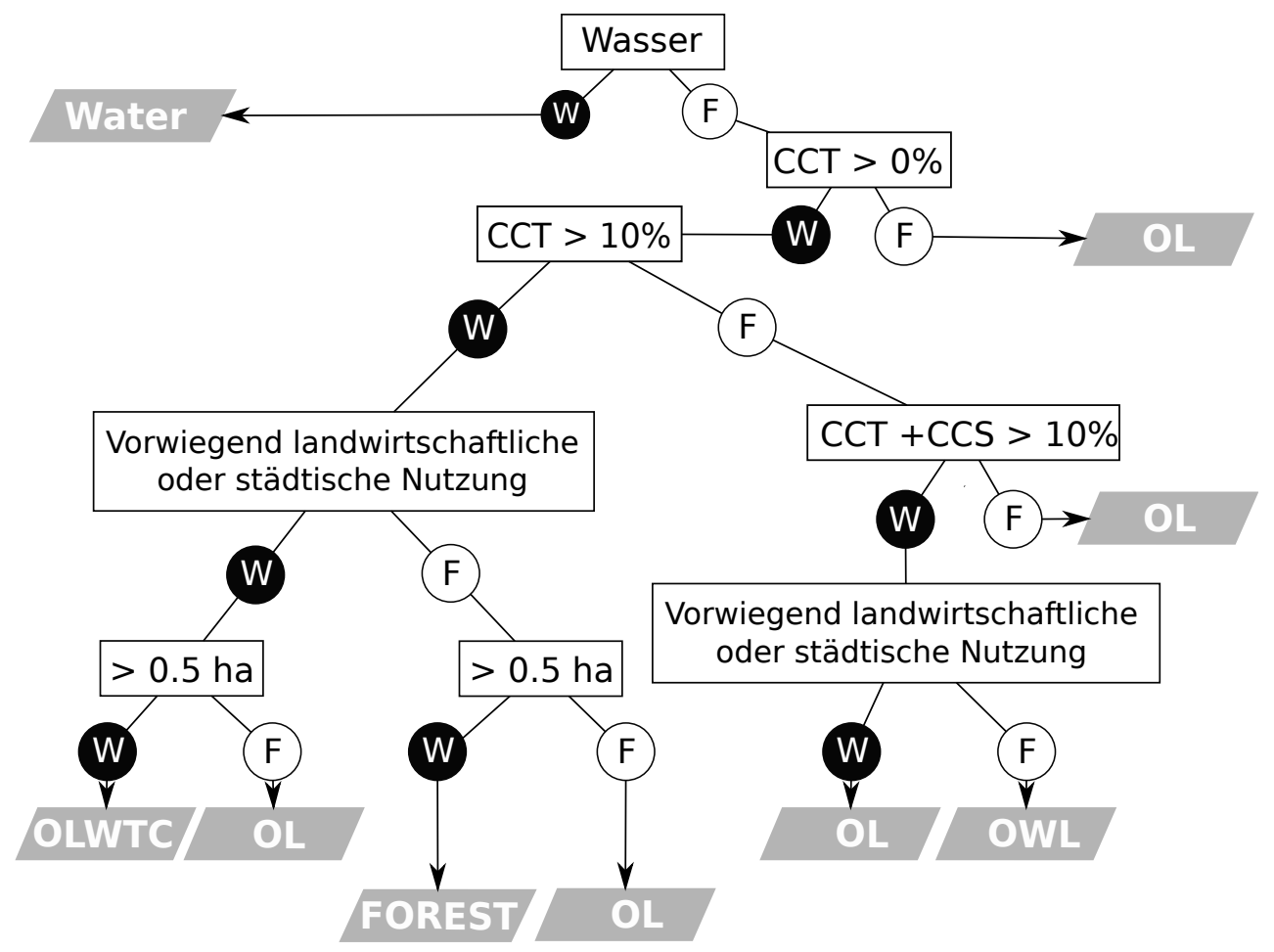

Abbildung 15.: Entscheidungsbaum zur Überführung der Level-I in die Level-II Klassen. Mit CCT: Baumkronenüberschirmung, CCS: Strauchüberschirmung, W: wahr und F: falsch. Eine Beschreibung der Klassen findet sich in Tabelle 6 . Quelle: Magdon et al. (2013), geändert. 


\section{Der ForestEye-Prozessor}

\subsection{Struktur und Aufbau}

Das in Kapitel 10 beschriebene Klassifikationsschema stellt einen neuen Ansatz in der Erstellung von Waldflächenkarten mit Hilfe von Fernerkundungsdaten dar. Die Umsetzung mit kommerziell verfügbaren Standardbildverarbeitungsprogrammen war daher nicht möglich. Deshalb wurde, in Zusammenarbeit mit Mitarbeitern der Abteilung Waldinventur und Fernerkundung der Universität Göttingen, eine eigene Software der -ForestEye-Prozessor (FEP) - entwickelt. Die Programmierung erfolgte in der Interactive Data Language (IDL) Version 8.0. Zur Vereinfachung der Programmpflege und Weiterentwicklung ist das Programm modular aufgebaut (siehe Abbildung 16). Es wurden Module für die typischen fernerkundlichen Arbeitsschritte der Bildklassifikation und zusätzliche Module zur Implementierung des FAO-Klassifikationsschlüssels entwickelt.

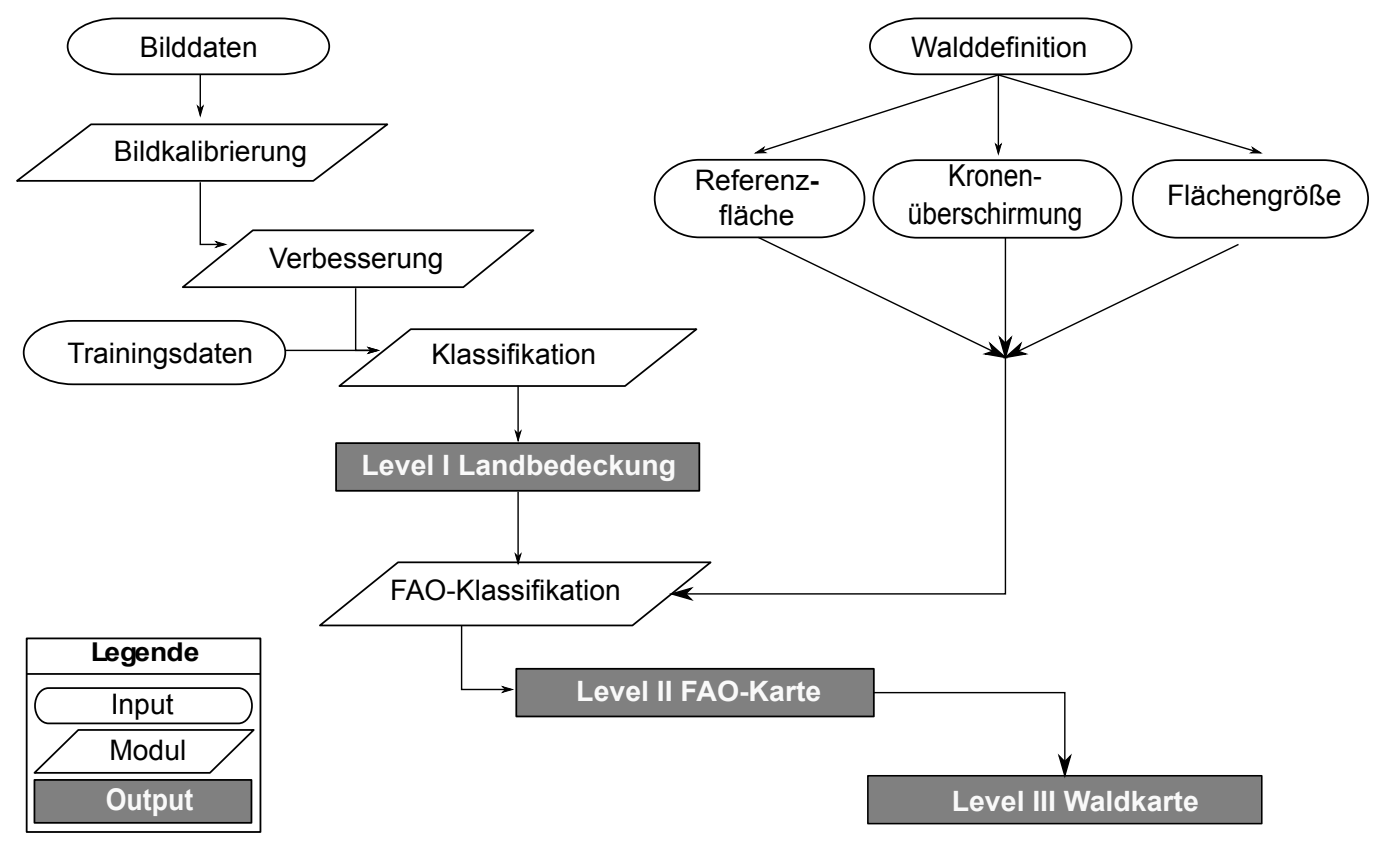

Abbildung 16.: Modularer Aufbau des ForestEye-Prozessors zum Erstellen der Landbedeckungskarten aus RapidEye-Daten gemäß der FAO-Walddefinition. 
Der ForestEye-Prozessor wurde speziell für die Auswertung von Bildern des RapidEyeSystems entwickelt. Als Input müssen neben den Bilddaten Grenzwerte für die Kriterien der Walddefinition (Mindestgröße, Mindestüberschirmung, Größe der Referenzfläche) gewählt werden. Danach durchlaufen die Bilddaten die verschiedenen Prozessierungsschritte und es werden drei Karten (Level-I, Level-II, Level-III) erstellt. Die Details der verschiedenen Prozessierungsschritte werden in den folgenden Abschnitten erläutert.

\subsection{Bildkalibrierung}

\subsubsection{Atmosphärenkorrektur}

Passive Fernerkundungssysteme, die elektromagnetische Energie im optischen Bereich des Sonnensprektrums erfassen (z. B. RapidEye), sind maßgeblich durch die Atmosphäre der Erde beeinflusst. Dieser Einfluss ist im Wesentlichen auf die Absorption und Reflexion der Strahlung an den Bestandteilen der Atmosphäre zurückzuführen. Beide Prozesse sind abhängig vom Zustand und der Zusammensetzung der Atmosphäre, welche permanenten Änderungen unterliegt. Dadurch unterscheiden sich die oberhalb der Atmosphäre am Sensor registrierten spektralen Strahlungsdichten $L_{*}$ zweier Aufnahmen auch dann, wenn keine Änderungen am Zielobjekt aufgetreten sind. Bei der Auswertung von Fernerkundungsdaten wird jedoch üblicherweise angenommen, dass die am Sensor registrierte Strahlung ausschließlich durch die biologischen, chemischen und physikalischen Eigenschaften der beleuchteten Objekte beeinflusst ist (Tso \& Mather, 2009). Vor der Analyse der Bilder sollte daher eine Kalibrierung durchgeführt werden, welche die atmosphärisch bedingten Variationen der Spektralsignaturen reduziert und eine Schätzung der spektralen Strahlungsdichte am Boden $L_{s}$ liefert. Beide Messungen $L_{*}$ und $L_{s}$ werden auch als dimensionsloses Verhältnis zwischen Sonneneneinstrahlung und registrierter Strahlung am Sensor (Top of Atmosphere Reflectance) $\rho *$ oder Reflexion am Boden (Surface Reflectance) $\rho_{s}$ angegeben. Zur Korrektur der Reflexion können zwei Ansätze genutzt werden:

1. empirische Angleichung verschiedener Aufnahmen durch Analyse von Bildbereichen mit konstant niedriger Reflexion (z. B. Wasserflächen)

2. physikalische Modellierung der Interaktion zwischen Strahlung und Atmosphäre mit Strahlungstransfermodellen 
Der zweite Ansatz hat den Vorteil, dass die Schätzung der Reflexion am Boden auf physikalischen Modellen basiert und somit mit anderen Messungen, z. B. Aufnahmen durch Feldspektrometer oder andere Sensoren, verglichen werden kann. In der vorliegenden Arbeit wurde das Strahlungstransfermodell Second Simulation of a Satellite Signal in the Solar Spectrum (6S) wie es in Vermote et al. (2006) beschrieben ist, verwendet. Das Modell wurde um eine neue Subroutine erweitert, welche die Filtertransmissionskurven der fünf RapidEye-Bänder im Bereich von 435-870nm hinterlegt. Eine vollständige Beschreibung der physikalischen Grundlagen des Strahlungstransfermodells findet sich in Tso \& Mather (2009) und Vermote et al. (2006, 1997). Hier soll nur eine kurze und vereinfachte Zusammenfassung erfolgen.

Das 6S-Modell ist ein additives Modell, das die Interaktion zwischen Strahlung und Atmosphäre in die Teilprozesse Absorption, Reflexion und Streuung zerlegt. Alle drei Prozesse hängen direkt von dem Weg der Strahlung zwischen Sonne, Erdoberfläche und Sensor ab. Daher wird die am Sensor registrierte Strahlung, wie in Abbildung 17 dargestellt, in fünf Teilmengen zerlegt.

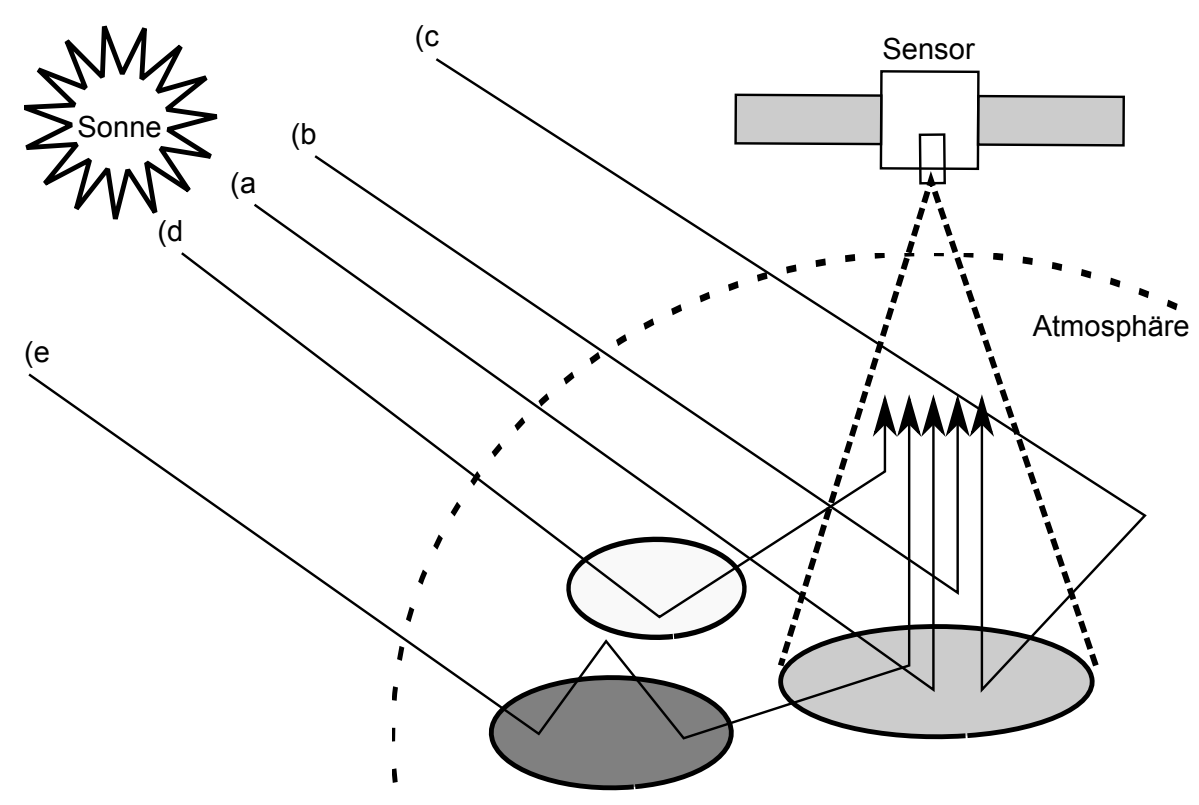

Abbildung 17.: Ansicht der verschiedenen Wege der Strahlung zwischen Sonne, Erde und Sensor nach Tso \& Mather (2009) (verändert) mit a) direkter Strahlung, b) atmosphärischer Strahlung, c) diffuser Strahlung, d) Überstrahlung und e) Trapping. Die Kreise stellen Flächen mit unterschiedlicher Landbedeckung dar. 
Der größte Teil der Sonnenstrahlung trifft nach Durchgang der Erdatmosphäre auf die Oberfläche, wird dort reflektiert und dann direkt am Sensor registriert (siehe Abbildung 17a). Dieser Anteil der Sonnenstrahlung, im Folgenden als $T\left(\theta_{s}\right)$ bezeichnet, ergibt sich aus $\tau$, der optischen Dicke und $\theta_{s}$, dem Sonnenzenitwinkel, wie in Gleichung 6 beschrieben. Ein Teil der in der Atmosphäre gestreuten Strahlung trägt indirekt auch zur Beleuchtung des Objektes in Form von diffuser Strahlung bei (Abbildung 17c) und wird im Folgenden als $t_{d}\left(\theta_{s}\right)$ bezeichnet.

$$
T\left(\theta_{s}\right)=\exp \left(\frac{-\tau}{\cos \left(\theta_{s}\right)}\right)+t_{d}\left(\theta_{s}\right)
$$

Abbildung 17e stellt einen als trapping bezeichneten Prozess dar. Hierbei wird die Strahlung mehrmals zwischen Boden und Atmosphäre reflektiert, bis sie vom Sensor erfasst wird. Somit wird am Sensor Strahlung gemessen, welche aus der Nachbarschaft des beobachteten Objektes stammt und durch Reflexion an der Atmosphäre zur Beleuchtung des Zielobjektes beiträgt. Dieser Prozess hängt vom Albedo der Atmosphäre $S$ und der Bodenreflexion $\rho_{s}$ ab, so dass sich der Anteil, der vom Boden reflektierten Strahlung, nach Gleichung 7 ergibt.

$$
\frac{\rho_{s}}{1-\rho_{s} S} \times T\left(\theta_{s}\right)
$$

Des Weiteren kann es zu Überstrahlungseffekten kommen, wenn Strahlung in der direkten Nachbarschaft des Objektes reflektiert und dann vom Sensor erfasst wird. Dieser Strahlungsanteil geht als $T\left(\theta_{v}\right)$ in das Strahlungsmodell ein. Er kann berechnet werden, indem man in der Gleichung 6 den Sensorzenitwinkel $\theta_{v}$ einsetzt. Als letzte Teilmenge ist der Teil der Strahlung, der den Boden nicht erreicht, sondern direkt in der Atmosphäre reflektiert und vom Sensor erfasst wird, zu berücksichtigen (Abbildung 17b). Dieser, als atmosphärische Strahlung $\rho_{a}$ bezeichnete Anteil, enthält keine Information über das Zielobjekt. Die atmosphärische Strahlung hängt von Sonnenzenit $\theta_{s}$, Sensorzenit $\theta_{v}$ und dem relativen Sonnenzenit $\phi$ ab. Die Releflexionssprozesse in der Atmosphäre können dann wie folgt zusammengefasst werden:

$$
\rho^{*}=\frac{\rho_{s}}{1-\rho_{s} S} \times T\left(\theta_{s}\right) \times T\left(\theta_{v}\right)+\rho_{a}\left(\theta_{s}, \theta_{v}, \phi\right)
$$


Neben den Reflexionsprozessen berücksichtigt das 6S-Modell auch die Absorption von Strahlung. Diese hängt hauptsächlich von der Sauerstoff-, Ozon-, Kohlendioxid-, Wasserdampfkonzentration und Aerosoldichte in der Atmosphäre ab, wobei die $\mathrm{H}_{2} \mathrm{O}$ und $\mathrm{O}_{3}$ Konzentrationen die höchste raum-zeitliche Variabilität zeigen. Das Modell aus Gleichung 8 wird daher um den Term $T_{g}\left(\theta_{s}, \theta_{v}\right)$ erweitert, welcher die Transmission nach der Absorption beschreibt.

$$
\left.\rho^{*}=T\left(\theta_{s}, \theta_{v}\right) \times \frac{\rho_{s}}{1-\rho_{s} S} \times T\left(\theta_{s}\right) \times T\left(\theta_{v}\right)+\rho_{a}\left(\theta_{s}, \theta_{v}, \phi\right)\right]
$$

Mit diesem Strahlungstransfermodell kann von der gemessenen Sensorreflexion $\rho^{*}$ auf die Bodenreflexion $\rho_{s}$ geschlossen werden. Dafür werden neben den verschiedenen Winkeln der Aufnahmegeometrie der atmosphärische Albedo $S$, der Anteil der atmosphärischen Strahlung $\rho_{a}$ und der Absorptionskoeffizient $T_{g}\left(\theta_{s}, \theta_{v}\right)$ benötigt, welche mit Hilfe des 6S-Modells berechnet werden können. Des Weiteren muss ein Atmosphärenmodell gewählt werden. Dieses beschreibt unter anderem Temperatur, Druck und chemische Zusammensetzung entlang eines Höhengradienten in der Atmosphäre. Zur Parametrisierung des 6S-Modells wurde das US-Standard-Atmosphärenmodell von 1962 verwendet (Sissenwine et al., 1962). Da die Konzentrationen von $\mathrm{H}_{2} \mathrm{O}$ und $\mathrm{O}_{3}$ stark schwanken, ist die Verwendung von Konstanten, wie sie im Atmosphärenmodell hinterlegt sind, nicht immer sinnvoll. Das Programm bietet aber die Möglichkeit die Konzentrationen individuell festzulegen, wenn diese z. B. aus anderen Beobachtungen ermittelt werden können (vergl. Abschnitt 13.2.2). Neben dem Atmosphärenmodell muss auch ein Aerosolmodell gewählt werden, das die Konzentration der verschiedenen Aerosole (z. B. Staub, Ruß) in der Atmosphäre beschreibt. Auch hierfür sind verschiedene Standardmodelle hinterlegt (z. B. typische Aerosolkonzentrationen für kontinental oder maritim geprägte Standorte). Der Sonnenzenitwinkel und die Flughöhe des Satelliten können aus den Metadaten der Bilder ausgelesen werden. Das 6S-Modell liefert somit für jede Aufnahme Schätzungen für die Parameter in Gleichung 9, so dass für jedes Bild ein spezifisches Strahlungstransfermodell parametrisiert werden kann.

Abbildung 18 fasst die einzelnen Arbeitschritte der atmosphärischen Bildkalibrierung zusammen. Im ersten Schritt werden die Pixelwerte in die absolute, kalibrierte, spektrale Strahlungsdichte am Sensor $L_{*}\left(\mathrm{~W} / \mathrm{m}^{2} / \mathrm{sr} / \mu \mathrm{m}\right)$ überführt. Dazu wird ein radiometrischer Skalierungsfaktor verwendet, der vom Anbieter der Bilder bezogen werden kann. Anschließend werden die Strahlungsdichten mit der Gleichung 10 ins Verhältnis zur Sonnenstrahlung 


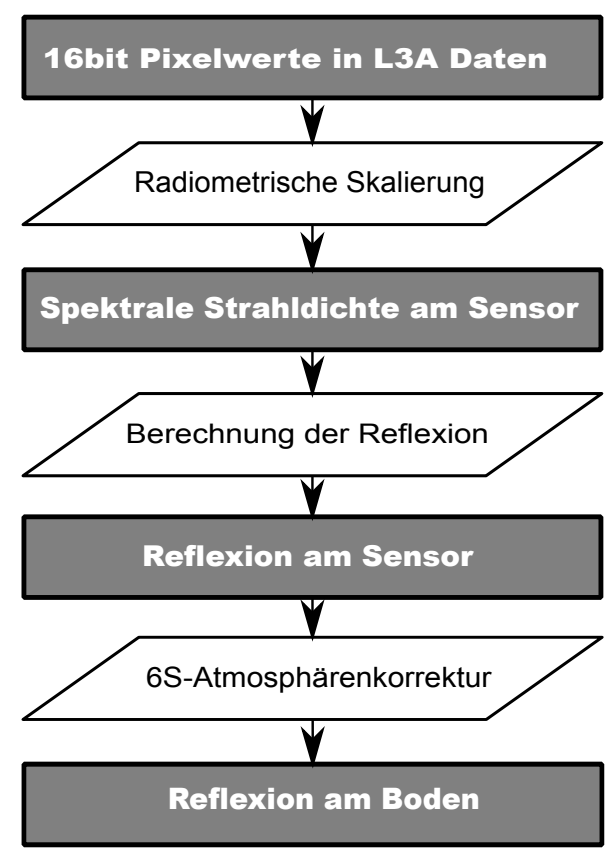

Abbildung 18.: Beispielhafter Ablauf zur atmosphärischen Korrektur von RapidEye-Daten

gesetzt, um die Reflexion am Sensor zu bestimmen (RapidEye AG, 2012).

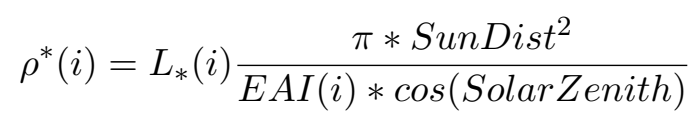

Die Berechnung der Reflexion am Sensor $\rho^{*}(i)$ erfolgt für jedes Band $i$ auf Grundlage des Abstands zur Sonne SunDist am Tag der Aufnahme, der exo-atmosphärischen Sonnenstrahlung EAI(i) und des Sonnenzenitwinkels SolarZenith. Im letzten Schritt werden die Parameterwerte des 6S-Modells und $\rho^{*}(i)$ in die Gleichung (9) eingesetzt und die Reflexion am Boden $\rho_{s}$ berechnet.

\subsubsection{Topographische Korrektur}

Durch die unregelmäßige Form der Erdoberfläche kann es zu starken Unterschieden in der Beleuchtung kommen, so dass die Reflexion in Abhängigkeit der Hangneigung und Exposition variiert. Zum Beispiel erscheinen Waldgebiete an Schattenhängen wesentlich dunkler als 
an Sonnenhängen. Dies kann die Klassifikation beeinträchtigen, da die Topographie zu einer Streuung der Spektralsignaturen der beobachteten Objekte führt. Die topographische Korrektur bietet die Möglichkeit, Beleuchtungsunterschiede, die durch die Hangneigung und Exposition verursacht sind, zu reduzieren. Prinzipiell können zwei Ansätze unterschieden werden:

1. die Bildung von Quotienten aus verschiedenen Bändern

2. die Modellierung der Beleuchtungssituation

Dem ersten Ansatz liegt die Annahme zugrunde, dass die topographisch bedingten Beleuchtungsunterschiede in allen Bändern proportional sind. Durch die Quotientenbildung aus zwei Bändern kann somit der topographische Effekt kompensiert werden. Nachteil dieser Methode ist, dass die spektrale Auflösung durch die Quotientenbildung reduziert wird, da für die Klassifizierung nur noch die Quotienten nicht aber die multispektralen Bänder zur Verfügung stehen. Daher wurde der zweite Ansatz, die Modellierung der Beleuchtungssituation, im FEP implementiert.

Im ersten Schritt zur Modellbildung wurde, wie in Riano et al. (2003) beschrieben, ein Beleuchtungskorrekturfaktor $I L$ für jedes Pixel berechnet. $I L$ beschreibt das Verhältnis zwischen einem geneigten Pixel und einem Pixel in der Ebene. Dieses Verhältnis ist abhängig von der Hangneigung $\theta_{p}$, dem Sonnenzenitwinkel $\theta_{z}$, dem Sonnenazimutwinkel $\phi_{a}$ und der Exposition $\phi_{o}$ :

$$
I L=\cos \left(\gamma_{i}\right)=\cos \left(\theta_{p}\right) * \cos \left(\theta_{z}\right)+\sin \left(\theta_{p}\right) * \sin \left(\theta_{z}\right) * \cos \left(\phi_{a}-\phi_{o}\right)
$$

Für die Korrektur der Reflexionswerte wurde das Lambertsche Strahlungsmodell genutzt. Es unterstellt, dass ein Objekt mit einer diffusen Oberfläche die Strahlung in alle Richtungen gleichmäßig reflektiert und somit aus jedem Betrachtungswinkel gleich hell erscheint. Daraus ergibt sich das Lambertsche Kosinusgesetz, welches besagt, dass die Strahlungsstärke proportional zum Kosinus des Betrachtungswinkels ist. Da die einfache Kosinuskorrektur oft zu einer Überkompensation der Beleuchtungsunterschiede führt, wurde die erweiterte Kosinuskorrektur von Cicvo (1989) implementiert, wie sie in Riano et al. (2003) beschrieben und in Gleichung 12 angegeben ist. Sie ermittelt für alle Reflexionswerte $\rho_{T}$ an der geneigten Oberfläche die erwarteten Reflexionswerte $\rho_{H}$ in der Ebene, indem sowohl die lokale 
Beleuchtung $I L$ als auch die mittlere Beleuchtung im Bild $I L_{m}$ berücksichtigt werden.

$$
\rho_{H}=\rho_{T}+\left[\rho_{T}\left(\frac{I L_{m}-I L}{I L_{m}}\right)\right]
$$

\subsection{Bildverbesserung}

\subsubsection{Vegetationsindices}

Die Verwendung von Vegetationsindices zur Verbesserung der Klassifikation ist weit verbreitet. Sie werden unter anderem eingesetzt, um die Vegetation von Nichtvegetation zu unterscheiden, Biomasseschätzungen abzuleiten oder die Vitalität von Pflanzen zu charakterisieren. Dazu werden aus den multispektralen Informationen durch Addition, Multiplikation und Division neue Kanäle berechnet, die sich die spezielle Reflexionscharakteristik von lebender Vegetation zunutze machen. Das RapidEye-System bietet neben dem nahen Infrarot (NIR) den RedEdge-Kanal, welcher im Wellenlängenbereich von 690-730nm sensitiv ist. In verschiedenen Studien konnte gezeigt werden, dass dieser Wellenlängenbereich empfindlich auf Veränderung der Pflanzenvitalität reagiert (Filella \& Penuelas, 1994) und somit zur einer verbesserten Landbedeckungsklassifikation führen kann (Förster et al., 2011; Zarco-Tejada \& Miller, 1999). Daher wurden für den FEP Routinen entwickelt, die neben den klassischen Vegetationsindices auch Indices berechnen, welche explizit die Informationen aus dem RedEdge-Kanal nutzen. Eine detaillierte Übersicht aller berechneter Vegetationsindices mit den entsprechenden Formeln und Referenzen findet sich in Tabelle 7.

\subsubsection{Texturindices}

Neben den Spektral- gehören Textur- und Kontextinformationen zu den wichtigsten Bildeigenschaften, die Menschen nutzen, um Bilder zu interpretieren (Haralick et al., 1973). Dies wird zum Beispiel bei der manuellen Interpretation von Graustufenbildern deutlich. Hier können aufgrund der Texturen häufig Objekte identifiziert werden, auch wenn keine Farbinformationen zur Verfügung stehten. Der Standard 610.4-1990 des Institute of Electrical and Electronics Engineers (IEEE) definiert Textur als ein Attribut, das die räumliche Anordnung der Grauwerte in einer Region beschreibt (IEEE, 1990). Bei der Auswertung digitaler Bilder 
Tabelle 7.: Vegetationsindices die mit dem ForestEye-Prozessor berechnet werden können.

\begin{tabular}{|c|c|c|}
\hline Name & Formel & Referenz \\
\hline NDVI & $N D V I=\frac{\mathrm{NIR}-\mathrm{ROT}}{\mathrm{NIR}+\mathrm{ROT}}$ & Campbell (2002) \\
\hline NDVI_RE & $N D V I_{R E}=\frac{\mathrm{NIR}-\mathrm{RE}}{\mathrm{NIR}+\mathrm{RE}}$ & Gitelson \& Merzlyak (1997) \\
\hline NDVI_GR & $N D V I_{G R}=\frac{\mathrm{NIR}-\mathrm{GRÜ \textrm {N }}}{\mathrm{NIR}+\mathrm{GR} \ddot{\mathrm{U} N}}$ & Gitelson \& Merzlyak (1997) \\
\hline RATIO & $R A T I O=\frac{N D V I_{R E}}{N D V I_{G R E E N}}$ & Marx (2010) \\
\hline Chlorophyll Green Model (CGM) & $C G M=\frac{\mathrm{NIR}}{\mathrm{GRÜN}}-1$ & Gitelson et al. (2005) \\
\hline Chlorophyll RedEdge (CRM) & $C R M=\frac{N I R}{R E-1}$ & Gitelson et al. (2005) \\
\hline
\end{tabular}

können statistische, modellbasierte und frequenzbasierte Verfahren genutzt werden, um die Textur in einer räumlichen Nachbarschaft zu beschreiben. Der FEP berechnet Texturindices nach zwei statistischen Verfahren, welche im Folgenden kurz erläutert werden.

Das erste Verfahren nutzt Statistiken erster Ordnung, um die Verteilung der Reflexionswerte in einer zuvor definierten Nachbarschaft zu charakterisieren. Die Nachbarschaft wird üblicherweise in Form eines quadratischen Beobachtungsfensters mit einer Seitenlänge von $X$ Spalten und $Y$ Zeilen definiert. Aus dieser Nachbarschaft werden alle Grauwerte extrahiert, eine Statistik berechnet und diese dem zentralen Pixel zugewiesen. Anschließend wird das Beobachtungssfenster um ein Pixel verschoben (Moving-Window-Operator) und erneut die jeweilige Statistik berechnet, bis für alle Pixel des Bildes die neuen Werte vorliegen. So entsteht ein neues Bild, welches die Verteilung der Grauwerte in der lokalen Nachbarschaft charakterisiert. Im FEP sind die in Tabelle 8 aufgelisteten Statistiken erster Ordnung implementiert.

Die Texturindices erster Ordnung quantifizieren die Verteilung der Grauwerte in der definierten Nachbarschaft. Eine Betrachtung der räumlichen Anordnung der Grauwerte ist jedoch nicht möglich. Daher ist das Ergebnis unabhängig von der Lage der Grauwerte und 
Tabelle 8.: Texturindices erster Ordnung, die mit dem ForestEye-Prozessor berechnet werden können.

\begin{tabular}{ll}
\hline Name & Abkürzung \\
\hline Mittelwert & AVG1 \\
Standardabweichung & STD1 \\
Variationskoeffizient & VAR1 \\
\hline
\end{tabular}

somit ungeeignet um Muster mit einer bestimmten Richtung, wie sie zum Beispiel durch Pflanzreihen in Plantagen entstehen, zu erfassen.

Haralick et al. (1973) entwickelten ein Verfahren zur Texturanalyse, welches die räumliche Anordnung der Grauwerte explizit berücksichtigt. Es basiert auf Statistiken zweiter Ordnung, welche nicht direkt aus den Grauwerten, sondern aus der Grauwertübergangsmatrix (engl.: Grey Level Co-Occurence Matrix, GLCM) abgeleitet werden. Die GLCM speichert die absoluten Häufigkeiten $P_{i j}$ mit denen eine Kombination der Grauwerte $i$ und $j$ in einer bestimmten Entfernung $d$ und Richtung $\theta$ in der definierten Nachbarschaft vorkommt. Abbildung 19 zeigt den Ablauf zur Erstellung der GLCM. Üblicherweise wird die GLCM vor der Berechnung der Statistiken auf den Wertebereich [0 - 1] normiert, indem die absoluten Häufigkeiten $P_{i j}$ durch einen Normierungsfaktor $R_{\theta}$ dividiert werden. Dieser ergibt sich. wie in Abbildung 19 dargestellt, aus der Größe der Nachbarschaft und dem Richtungswinkel $\theta$. Letztendlich müssen bei der Berechnung der GLCM vier Parameter definiert werden:

i) Größe der Nachbarschaft

ii) Spektrale Auflösung $G$

iii) Richtung $\theta$

iv) Verschiebung $d$.

Haralick et al. (1973) definierten die Nachbarschaft für die Texturberechnung in Form von überlappenden Blöcken. Zum Beispiel unterteilte er eine MSS Landsatszene $(2340 \times 3200 p x)$ in 624 Blöcke á $64 \times 64 p x$. Für diese Blöcke wurden anschließend die Texturindices berechnet und eine Klassifizierung durchgeführt. Bei diesem Vorgehen reduziert sich die räumliche Auflösung der Texturindices um den Faktor 64. Der FEP verwendet keine Blöcke, sondern definiert die Nachbarschaft in Form eines Moving-Window-Operators. Dieser ermöglicht die Berechnung der GLCM für jedes Pixel, womit die originäre Auflösung erhalten bleibt. Die spektrale Auflösung des Grauwertbildes $G$ ist ein weiterer zu bestimmender Parameter, da die Größe der GLCM nach $N_{g}=G \times G$ bestimmt wird. Daraus ergibt sich, dass in 


Bild mit $N_{g}=2^{2}$ Grauwertstufen
\begin{tabular}{|c|c|c|c|c|}
\hline 1 & 0 & 1 & 3 & 3 \\
\hline 0 & 0 & 1 & 0 & 0 \\
\hline 0 & 3 & 2 & 2 & 2 \\
\hline 2 & 2 & 3 & 3 & 3 \\
\hline 3 & 0 & 3 & 2 & 3 \\
\hline
\end{tabular}

\begin{tabular}{|c|c|c|c|c|c|c|c|}
\hline \multicolumn{4}{|c|}{ GLCM } & \multicolumn{4}{|c|}{ Normalisierte GLCM } \\
\hline 2 & 3 & 0 & 1 & 0.17 & 0.25 & 0 & 0.08 \\
\hline 3 & 0 & 0 & 0 & 0.25 & 0 & 0 & 0 \\
\hline 0 & 0 & 0 & 1 & 0 & 0 & 0 & 0.08 \\
\hline 1 & 0 & 1 & 0 & 0.08 & 0 & 0.08 & 0 \\
\hline
\end{tabular}

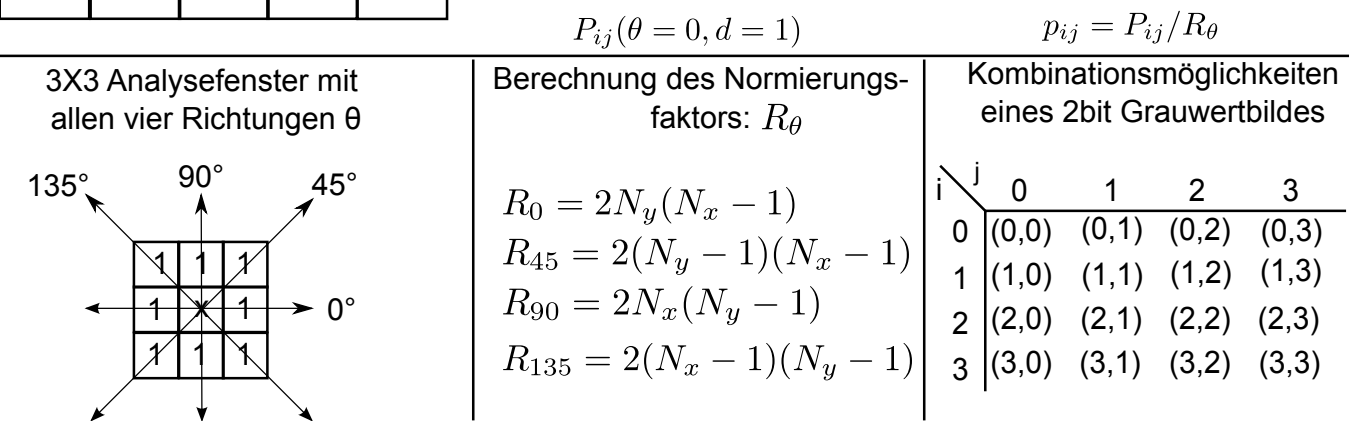

Abbildung 19.: Schema zur Ableitung und Normalisierung einer Grauwertübergangsmatrix (GLCM) aus einem 2bit Grauwertbild für $\theta=0^{\circ}$ und $d=1$.

12bit Daten für jedes Pixel eine GLCM mit den Dimensionen $2^{12} \times 2^{12}=4096 \times 4096$ berechnet werden müsste. Da die Wahrscheinlichkeit, dass Grauwerte mehrmals vorkommen mit zunehmender Anzahl der Grauwertstufen sinkt, sind die meisten Felder einer so großen GLCM mit sehr kleinen bzw. mit Nullwerten besetzt. Clausi (2002) untersuchte den Einfluss der Grauwertstufenanzahl bei der Klassifikation von Radardaten und konnte zeigen, dass eine Anzahl von $G>24$ Grauwertstufen ausreichend ist und bei einer Erhöhung der Anzahl auf $G>64$ kaum noch Verbesserungen zu erwarten sind. Mit Rücksicht auf den hohen Rechenaufwand, ist es daher sinnvoll, die Anzahl der Grauwertstufen für die Berechnung der GLCM zu reduzieren. Der dritte wichtige Parameter für die Berechnung der GLCM ist die Richtung $\theta$. Es können GLC-Matrizen für bestimmte Richtungen oder für eine Kombination aus mehreren Richtungen berechnet werden. In Tabelle 9 sind alle 11 Texturindices zweiter Ordnung aufgeführt, die in der vorliegenden Arbeit berechnet wurden. 
Tabelle 9.: Texturindices zweiter Ordnung mit $G$ : Anzahl der Grauwertstufen; $p_{i, j}$ : Wahrscheinlichkeit aus der normierten GLCM; $\mu_{x}, \mu_{y}$ und $\sigma_{x}, \sigma_{y}$ : Mittelwerte und Standardabweichung der Reihe $i$ und Spalte $j$ der GLCM.

\begin{tabular}{lll}
\hline Name & Formel & Referenz \\
\hline Angular Second Moment & $A S M=\sum_{i, j=1}^{G} p_{i j}^{2}$ & Haralick et al. (1973) \\
Contrast & $C O N=\sum_{i, j=1}^{G} p_{i j}(i-j)^{2}$ & Haralick et al. (1973) \\
Entropy & $E N T=\sum_{i, j=1}^{G} p_{i j} \log p_{i j}$ & Haralick et al. (1973) \\
Inverse Difference Moment & $I D M=\sum_{i, j=1}^{G}\left(\frac{1}{\left.1+(i-j)^{2}\right)} p_{i j}\right.$ & Haralick et al. (1973) \\
Correlation & $C O R=\frac{\sum_{i, j=1}^{G} p_{i j}-\mu_{x} \mu_{y}}{\sigma_{x} \sigma_{y}}$ & \\
Dissimilarity & Haralick et al. (1973) \\
Cluster Shade & DIS $=\sum_{i, j=1}^{G} p_{i j}|i-j|$ & Clausi (2002) \\
Cluster Prominence & PRO $=\sum_{i, j=1}^{G}\left(i+j-\mu_{i}-\mu_{j}\right)^{3} p_{i j}$ & Conners et al. (1984) \\
Maximum Probability & $M A X 2=\sum_{i, j=1}^{G}\left(i+j-\mu_{i}-\mu_{j}\right)^{4} p_{i j}$ & Conners et al. (1984) \\
Mean & $A V G 2=\operatorname{Mean}\left(p_{i j}\right)$ & - \\
Variance & $V A R 2=\operatorname{Variance}\left(p_{i j}\right)$ & - \\
\hline
\end{tabular}

\subsection{Random Forests Klassifikation}

Die Klassifikation der Bilder überführt die physikalischen Messungen $\rho_{s}$ in thematische Klassen und stellt somit die Grundlage für die Erstellung von Landbedeckungskarten dar. Zur Klassifikation stehen unzählige Verfahren mit spezifischen Vor- und Nachteilen zur Verfügung. Für die Auswahl eines geeigneten Verfahrens wurden zunächst folgende Anforderungen formuliert:

- Es soll nicht-parametrisch sein, um ohne restriktive Verteilungsannahmen eine große Bandbreite von Bilddaten verarbeiten zu können. 
- Es soll ein Modell liefern, welches auf neue Bilddatensätze angewendet werden kann.

- Es soll die Möglichkeit bieten, verschiedene Trainingsdatensätze zu kombinieren.

- Es soll die Möglichkeit bieten, die Bedeutung der Prädiktoren zu bestimmen.

- Es soll möglichst effizient in Bezug auf die erforderlichen Berechnungszeiten sein, so dass auch sehr große Datensätze prozessiert werden können.

Aufgrund dieser Anforderungen fiel die Wahl auf ein Verfahren des maschinellen Lernens, den Random Forests (RF) Klassifikator, welcher von Breiman (2001) entwickelt wurde. Dieser erfüllt die genannten Voraussetzungen weitgehend und wurde auch in anderen fernerkundlichen Studien mit forstlichen Fragestellungen erfolgreich eingesetzt (Falkowski et al., 2009; Houghton et al., 2007; Immitzer et al., 2012; Main-Knorn et al., 2011; Powell et al., 2010). Im Folgenden wird das Verfahren kurz erläutert.

Der RF-Klassifikator kann als Weiterentwicklung der ursprünglichen Arbeiten von Breiman et al. (1984) zu Klassifikations- und Entscheidungsbäumen (engl. Classification and Regression Trees, CART) gesehen werden. Breiman (2001) definiert den RF-Klassifikator als eine Sammlung von Entscheidungsbäumen $h\left(x, \theta_{k}\right)$ mit $\theta_{k}$ unabhängig und identisch verteilten Zufallsvektoren. Die Klassifikation von $x$ ergibt sich als Mehrheitsentscheidung aus den einzelnen Entscheidungsbäumen. Dieses Verfahren gehört somit zur Gruppe der kombinierten Klassifikatoren (engl. ensemble-techniques), die dadurch charakterisiert sind, dass sie mehrere unabhängige Klassifikationen miteinander kombinieren. Dahinter steht die „demokratische“ Idee, dass die Qualität einer Entscheidung durch die Kombination mehrerer Vorhersagen/Meinungen verbessert wird. Im Fall des RF-Klassifikators werden mehrere unabhängige Entscheidungsbäume kombiniert.

Der Aufbau der einzelnen Entscheidungsbäume ist für das Verständnis der Funktionsweise des RF-Klassifikators wichtig und soll daher kurz erläutert werden. Ein Entscheidungsbaum kann als eine hierarchische Sequenz von Entscheidungen verstanden werden. Der Entscheidungsprozess verläuft entlang von Zweigen, welche über Knoten verbunden sind und endet am Endknoten, welcher häufig auch als Blatt bezeichnet wird. An oberster Stelle des Baums steht die Population aller zu klassifizierenden Objekte. Durch eine erste Entscheidung wird die Population in zwei Subpopulationen aufgeteilt, so dass zwei neue Zweige entstehen. Wenn damit alle Elemente der Subpopulation einer Klasse zugeordnet werden können, endet der Zweig mit einem Endknoten. Ansonsten folgt eine neue Verzweigung, welche die Subpopulation weiter aufteilt. Die Aufteilung wird solange fortgesetzt bis alle Zweige in einem Endknoten enden und somit jedes Element der Ausgangspopulation einer Klasse zu- 
geordnet ist, wie in Abbildung 20 dargestellt.

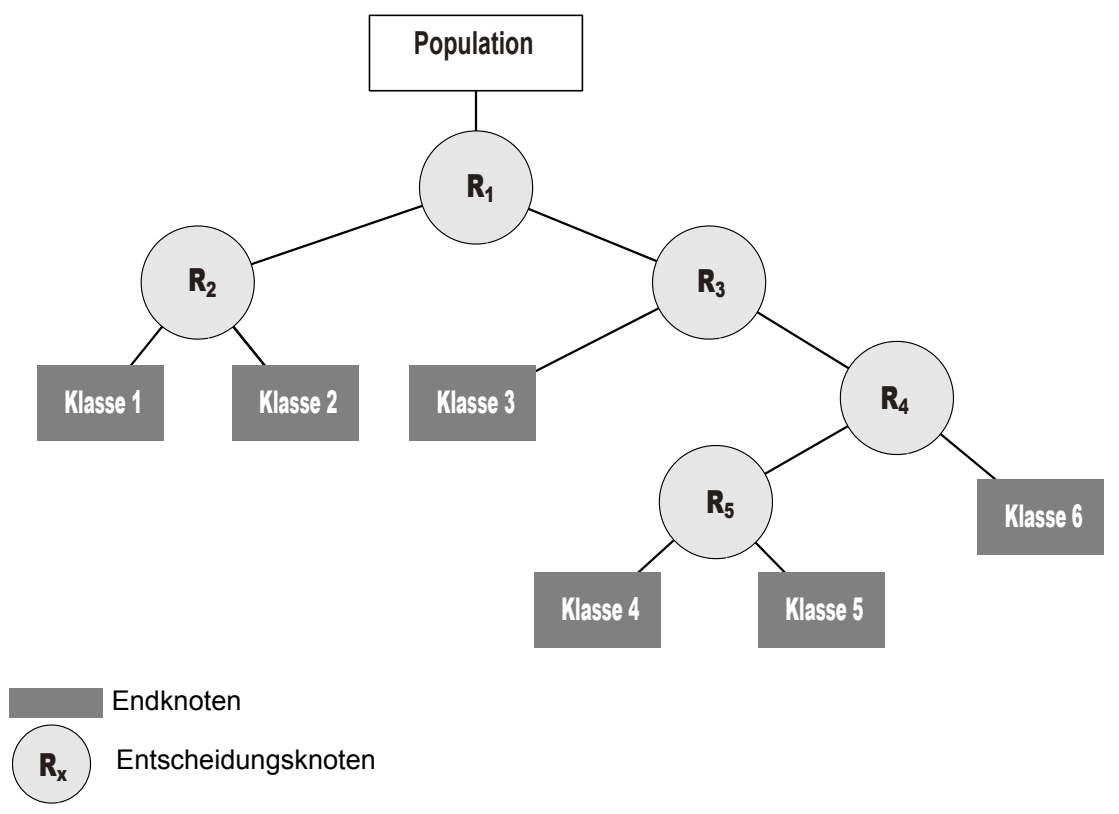

Abbildung 20.: Schematische Darstellung eines Entscheidungsbaums

Um einen Entscheidungsbaum $h_{k}(x)$ aufzubauen, muss an jeder Verzweigung ein Attribut $i \in m$ aus der Liste der zur Verfügung stehenden Attribute $m$ ausgewählt werden. Dabei sollte das Attribut gewählt werden, welches die Population am effizientesten aufteilt. Eine Entscheidung ist dann effizient, wenn sie möglichst viele Elemente der Population klassifiziert, also einem Endknoten zuweist, da so die Anzahl der nötigen Entscheidungen verringert wird. Zur Beurteilung der Effizienz kommen verschiedene Statistiken in Frage. Der RF-Klassifikator verwendet den Gini-Impurity-Index zur Beurteilung der Entscheidungseffizienz. Dieser berechnet sich aus den Wahrscheinlichkeiten $p_{i}$ der Klassen $i$ wie folgt:

$$
I_{G}=1-\sum_{i=1}^{m} p_{i}^{2}
$$

Der RF-Klassifikator $h(x)$ kombiniert mehrere so erzeugte Entscheidungsbäume $h_{k}(x)$, indem die Klassifikationen der einzelnen Entscheidungsbäume zusammengefasst werden. Dafür wird die Verteilung der Ergebnisse aller Entscheidungsbäume für jedes Objekt untersucht und die Klasse, welche am häufigsten zugewiesen wurde, als endgültige Klasse gewählt 
(Mehrheitsentscheid). Nach Breiman (2001) hängt die Genauigkeit des Verfahrens von der Stärke der einzelnen Endscheidungsbäume und deren Korrelation ab. Details zur Berechnung der Stärke und Korrelation der Entscheidungsbäume finden sich in Breiman (2001). Es gilt, dass starke Entscheidungsbäume mit einer schwachen Korrelation die höchste Klassifikationsgenauigkeit ergeben. Um eine möglichst geringe Korrelation der Entscheidungsbäume zu gewährleisten, werden zwei verschiedene Randomisierungstechniken eingesetzt. Zunächst wird das bagging-Verfahren genutzt. Dieses selektiert, für jeden Entscheidungsbaum $h_{k}$ aus dem gesamten Trainingsdatensatz $\theta$ mit $N$ Elementen per Zufallsauswahl (Ziehen mit Zurücklegen) einen neuen Trainingsdatensatz $\theta_{k}$ mit $n<N$ Elementen. Dieser wird genutzt, um den Entscheidungsbaum zu trainieren. Somit verwendet jeder Entscheidungsbaum einen unabhängigen Trainingsdatensatz mit gleicher Verteilung. Neben der Verbesserung der Klassifikationsgenauigkeit ermöglicht das bagging-Verfahren außerdem deren kontinuierliche Schätzung. Dies ist möglich, weil die Elemente aus dem vollständigen Trainingsdatensatz $\theta$, die nicht für den Trainingsdatensatz $\theta_{k}$ ausgewählt wurden (engl. OutOf-Bag, OOB), für die Berechnung des Klassifikationsfehlers zur Verfügung stehen. Mit den so ausgewählten Trainingsdaten wird der Entscheidungsbaum aufgebaut. Um die Korrelation der Entscheidungsbäume zu reduzieren, erfolgt dabei ein zweiter Randomisierungschritt, indem an jedem Entscheidungsknoten nicht alle $M$ Attribute des Trainingsdatensatzes, sondern nur $m<M$ für die Auswahl zur Verfügung stehen. Dadurch wird die Unabhängigkeit der Entscheidungsbäume erhöht, da sich die Ergebnisse der Klassifikation auch bei gleichem Trainingsdatensatz $\theta_{k}$ geringfügig unterscheiden.

Auswahl der Prädiktoren Die Auswahl der Prädiktoren ist sinnvoll, um den Klassifikationsfehler zu reduzieren, den Klassifikationsaufwand zu minimieren und die Klassifikationsmodelle so einfach wie möglich zu gestalten (Guyon \& Elisseeff, 2003). Ein wesentlicher Vorteil des RF-Klassifikators ist die Möglichkeit, die Bedeutung der verwendeten Prädiktoren zu bewerten. Im Gegensatz zu univariaten Verfahren können hier sowohl die einzelnen Prädiktoren als auch deren multivariate Interaktion analysiert werden, auch dann, wenn sie korrelieren (Strobl et al., 2007). Dies ist bei der Auswertung von Fernerkundungsdaten besonders wichtig, da die einzelnen Bänder häufig stark miteinander korrelieren. Drei Statistiken zur Beurteilung der Prädiktorenbedeutung (engl. variable importance=VI) können aus der RF-Klassifikation abgeleitet werden:

1. Häufigkeit, mit der ein bestimmtes Attribut in allen $n$ Entscheidungsbäumen selektiert wurde (Auswahlwahrscheinlichkeit) 
2. Gini Variable Importance (GVI)

3. Permutation Variable Importance (PVI)

In dieser Arbeit wird zur Bewertung der Prädiktoren die PVI genutzt. Wie der Name schon andeutet, werden zur Berechnung die Werte des jeweiligen Attributes zufällig vertauscht. Die Berechnung erfolgt dann in sechs Schritten (Breiman \& Cutler, 2012):

1. Berechnung der Anzahl korrekt klassifizierter Objekte aus den OOB-Daten für jeden Entscheidungsbaum

2. Permutation: Vertauschen der Werte des zu untersuchenden Attributes $m$ und anschließende Berechnung der Anzahl korrekt klassifizierter Objekte

3. Bildung der Differenz zwischen beiden Anzahlen

4. Berechnung des Mittelwerts der Differenzen über alle $n$ Entscheidungsbäume ergibt die raw importance

5. Berechnung des Standardfehlers der raw importance

6. Der Quotient aus dem raw importance und dem Standardfehler ergibt den Z-Score, für den unter der Annahme der Normalverteilung eine Signifikanzwert $p$ bestimmt wird.

\subsection{Evaluation der Klassifikationsergebnisse}

Für die Bewertung der Klassifikation muss zwischen zwei Fehlerarten unterschieden werden:

Modellfehler Der Modellfehler beschreibt die Qualität des parametrisierten Modells indem die Prognosen der Modelle den bekannten Klassen des Trainingsdatensatzes gegenüber gestellt werden. Der RF-Klassifikator nutzt das bagging-Verfahren, weshalb die Berechnung des Modellfehlers auf Basis der OOB-Werte erfolgen kann.

Klassifikationsfehler Bei der Anwendung des Klassifikationsmodells auf unbekannte Objekte, die nicht für die Parametrisierung der Modelle verwendet wurden, können Klassifikationsfehler auftreten. Übertragen auf die fernerkundliche Anwendung kann es zu Fehlklassifikationen der Pixel kommen, bei denen sich der prognostizierte Wert vom wahren Wert unterscheidet. Der Klassifikationsfehler beschreibt diese Abweichung zwischen Modell und 
Realität. In vielen Fällen ist der Modellfehler (z. B. OOB-Fehler) kein geeigneter Schätzer für den Klassifikationsfehler. Eine Möglichkeit zur Bestimmung des Klassifikationsfehlers ist die Verwendung eines unabhängigen Datensatzes. Die Klassen der Klassifikation und des Validierungsdatensatzes können in einer Fehlermatrix gegenübergestellt werden. Aus der Fehlermatrix lassen sich dann die Gütemerkmale: Herstellergenauigkeit, Nutzergenauigkeit, Gesamtgenauigkeit und der Kappawert berechnen. Details, wie diese Gütemerkmale berechnet werden, finden sich in fast allen Fernerkundgslehrbüchern (z. B. Campbell (2002)) und werden daher hier nicht weiter erläutert. 

Teil IV.

Fallstudie: Costa Rica 



\section{Ausgangssituation}

Im vierten Teil der Arbeit soll anhand einer Fallstudie geprüft werden, ob das entwickelte Verfahren geeignet ist, unter realistischen Bedingungen standardisierte Waldkarten zu erstellen. Dazu werden für zwei unterschiedliche tropische Landschaften in Costa Rica mit dem ForestEye-Prozessor Waldkarten erstellt. Costa Rica eignet sich besonders als Untersuchungsgebiet für die Entwicklung eines Waldflächenmonitorings, da sowohl die Waldflächenausdehnung als auch die räumliche Verteilung der Waldfläche stark schwanken. Dieser dynamische Prozess war Gegenstand mehrerer Untersuchungen, welche in Kleinn et al. (2002) zusammengefasst sind. Insgesamt kann für Costa Rica eine typische Waldentwicklungskurve (forest transition curve) mit zunächst sehr starkem Rückgang der Waldfläche zwischen 1960 und 1980 und anschließender Zunahme beobachtetet werden (Kanninen et al., 2007). Als Grundlage für die Klassifikation werden Satellitenbilder des RapidEye-Systems verwendet. Die Bewertung der erstellten Karten erfolgt auf drei Ebenen. Die Modellfehler der RF-Klassifikationsmodelle werden mit dem OOB-Fehler beschrieben. Die Kartengenauigkeit wird auf Basis eines unabhängigen Validierungsdatensatzes bestimmt und in Form von Fehlermatrizen angegeben. Abschließend erfolgt ein Vergleich der aus der Klassifikation abgeleiteten mit der im Feld gemessenen Kronenüberschirmung. 



\section{Untersuchungsgebiete und Bildmaterial}

\subsection{Beschreibung der Untersuchungsgebiete}

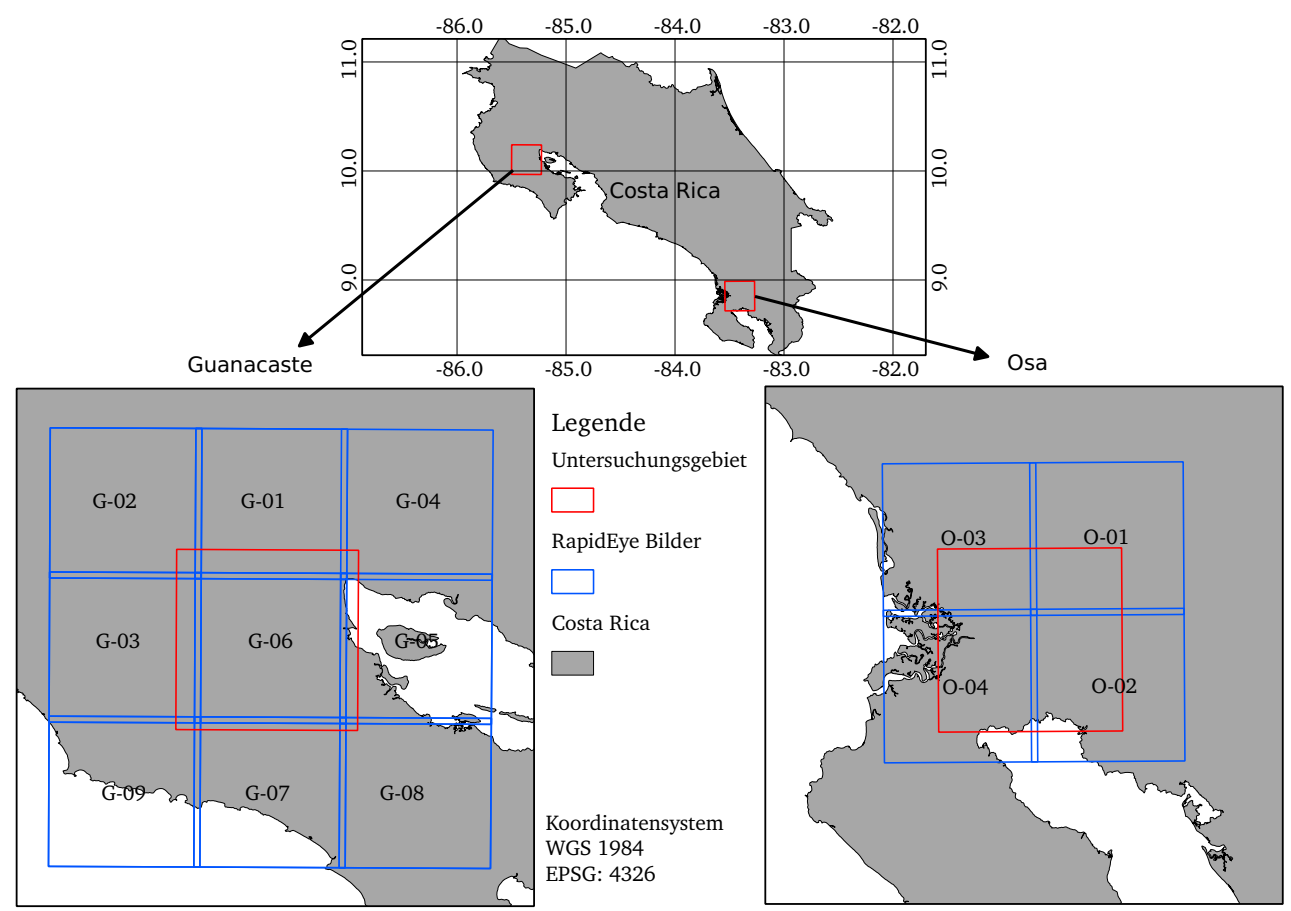

Abbildung 21.: Lage der beiden Untersuchungsgebiete Osa und Guanacaste sowie Darstellung der RapidEye-Abdeckungen und Bildnummern, wie in Tabelle 11 angegeben.

Verschiedene Kriterien wurden bei der Auswahl der Untersuchungsgebiete in Costa Rica berücksichtigt. Es sollten möglichst verschiedene tropische Waldtypen untersucht werden, um zu prüfen ob das Klassifikationsverfahren auf verschiedene Waldtypen anwendbar ist. Ein weiteres Auswahlkriterium war die Fragmentierung der Landschaft. Es wurden bewusst Landschaftsausschnitte mit verschieden stark fragmentierten Wäldern gewählt. Neben diesen Kriterien spielten auch praktische und logistische Argumente eine Rolle. Basierend auf den genannten Kriterien wurden zwei Untersuchungsgebiete mit einer Größe von $30 \mathrm{~km} \times 30 \mathrm{~km}$ angelegt (siehe Abbildung 21), die im Folgenden kurz beschrieben werden. 
Osa Das südliche Untersuchungsgebiet schließt Teile der Halbinsel Osa ein und befindet sich an der Grenze zu Panama. Im Norden wird das Gebiet durch eine Bergkette, im Süden durch den Pazifik geprägt, sodass Höhen zwischen 0-1081m auftreten. Das Untersuchungsgebiet erstreckt sich über die Distrikte Palmar, Sierpe und Piedras Blancas und zeigt eine für Zentralamerika typische, fragmentierte Landschaft, die dem Relief folgt. Im zentralen und nördlichen flachen Teil überwiegt die landwirtschaftliche Nutzung. Hier werden unter anderem Reis, Bananen und Ölpalmen angebaut. Verstreut finden sich einzelne kleine Holzplantagen, vorwiegend mit Melina- (Gmelina arborea) und Teakbeständen (Tectona grandis). Im südlichen bergigen Teil sind noch größere zusammenhängende Waldgebiete mit feuchttropischen Regenwäldern vorhanden. Im Westen dominieren große Mangroven-Gebiete. Das Untersuchungsgebiet befindet sich in der Kernzone des ACOSA-Schutzgebietes (Área de Conservación Osa) und hat aufgrund seiner Lage auf der pazifischen Seite von Costa Rica einen Jahresniederschlag von ca. $5600 \mathrm{~mm}$ bei einer Jahresmitteltemperatur von ca. $27^{\circ} \mathrm{C}$ (vergleiche Abbildung 24).

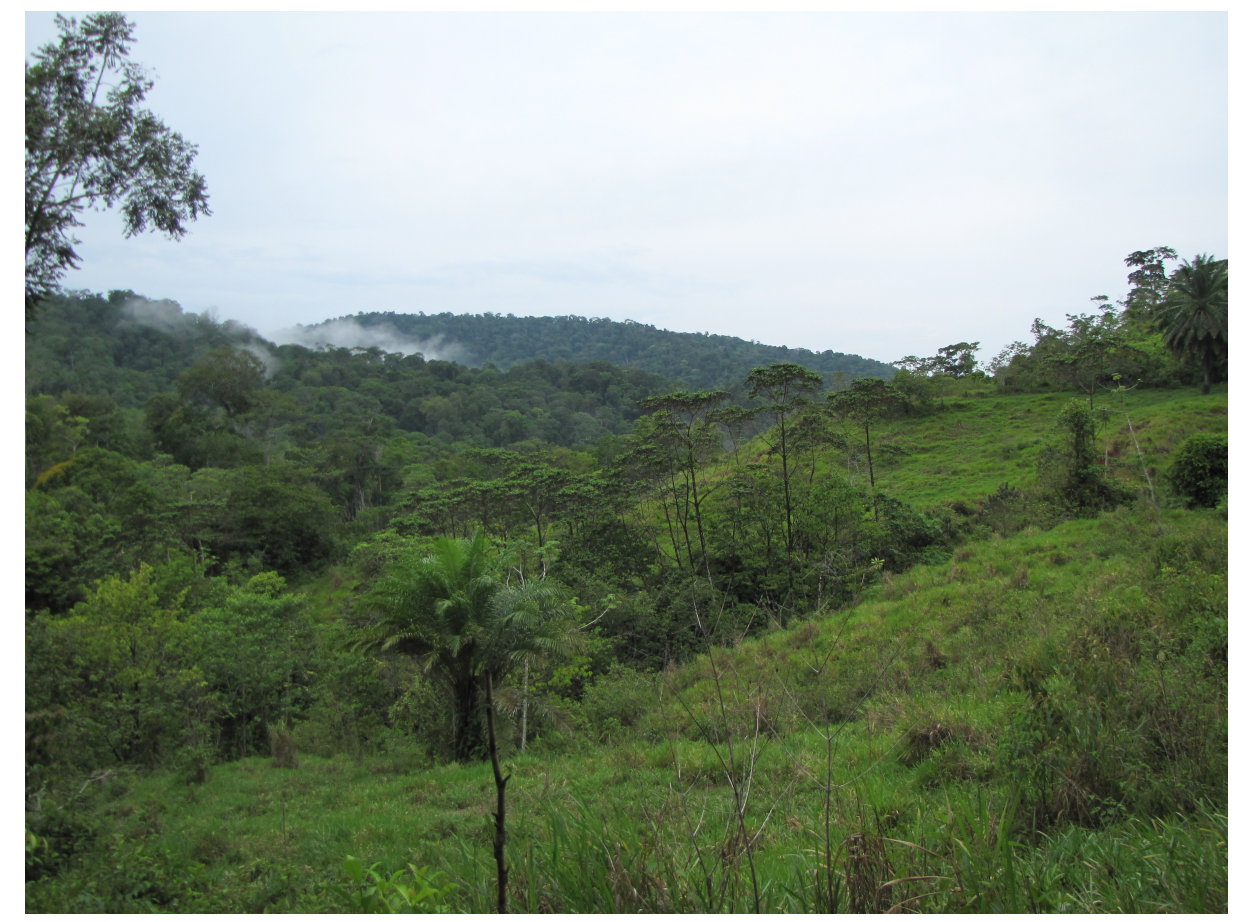

Abbildung 22.: Typisches Landschaftsbild in Osa. Aufgenommen am 13.04.2010. Im Vordergrund zeigen sich einige aufgegebene Weideflächen mit einsetzender Sukzession. Im Hintergrund sind dichte Baumbestände zu erkennen. 
Guanacaste Das zweite Untersuchungsgebiet liegt im Norden von Costa Rica auf der Nicoya Halbinsel. Die gesamte Halbinsel hat ein sehr stark ausgeprägtes Relief, welches die Landnutzung prägt. Entlang der Flussufer, wo eine Bewässerung möglich ist, wird teilweise Acker- und Obstbau betrieben. Hier werden Kürbisse und andere Feldfrüchte angebaut. An den flacheren Hängen finden sich hauptsächlich Viehweiden für Rinder. In den höheren Berglagen wird Kaffee angebaut. Größere Waldflächen finden sich nur noch in Schutzgebieten und entlang der steilen Hänge, wo eine andere Landnutzung kaum möglich ist. Der mittlere Jahresniederschlag beträgt $1880 \mathrm{~mm}$ bei einer Jahresmitteltemperatur von ca. $27^{\circ} \mathrm{C}$.

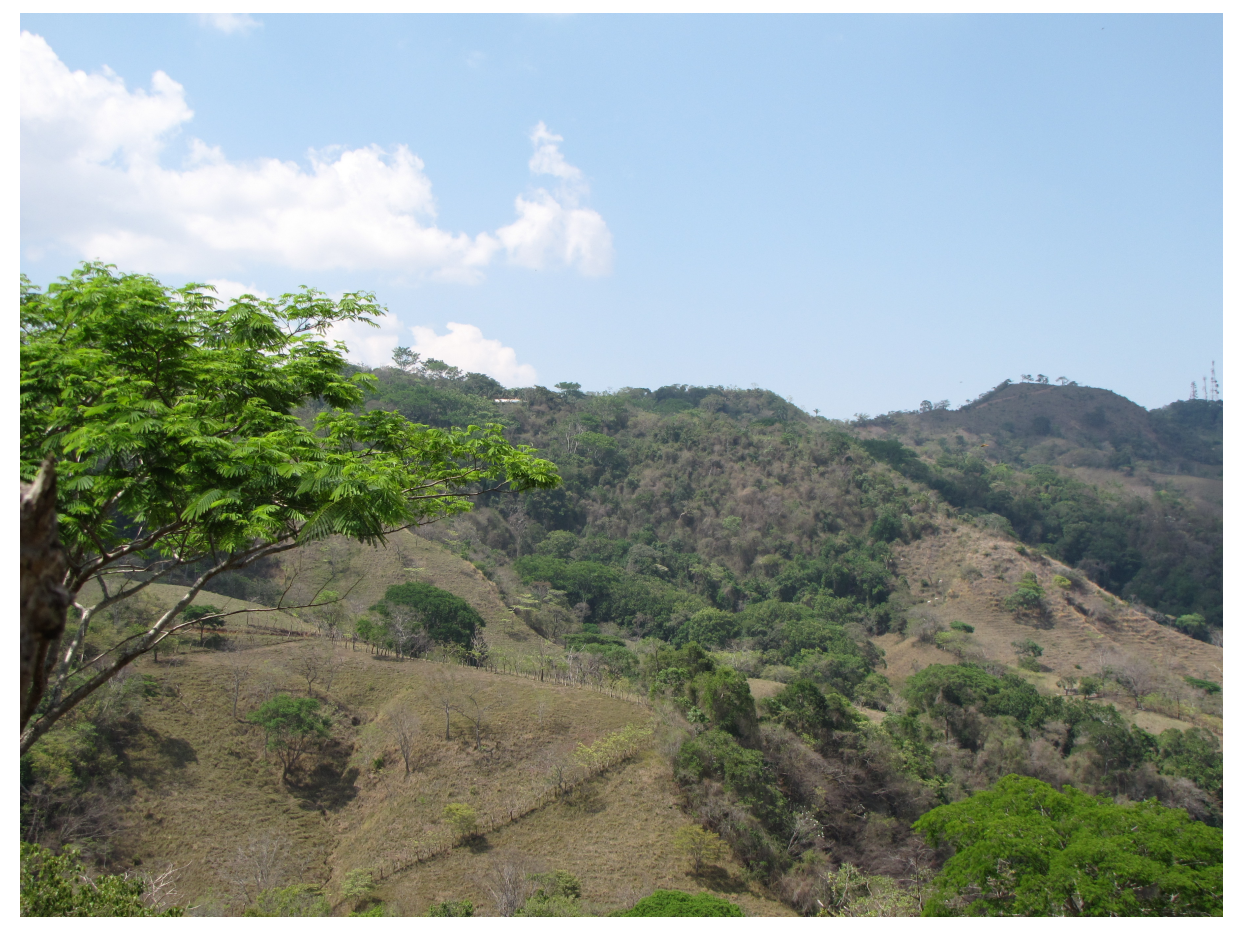

Abbildung 23.: Typisches Landschaftsbild in Guanacaste. Aufgenommen am 18.04.2010 zu Beginn der Regenperiode. Entlang der Hänge befinden sich intensiv genutzte Viehweiden; in den Tälern kommen Fragmente von Baumgruppen vor, die teilweise noch unbelaubt sind.

Wie in Abbildung 24 dargestellt, ist Guanacaste, im Gegensatz zu Osa, durch starke jahreszeitliche Schwankungen der Niederschläge geprägt. In der Trockenperiode, von November bis April, fallen bei anhaltend hohen Temperaturen kaum Niederschläge. Aufgrund dieses besonderen Klimas finden sich in der Region Wälder, die nach der Holdridge LebenszonenKlassifikation (Holdridge, 1967) zu den tropischen Trockenwäldern gehören. Sie zählen zu den am stärksten gefährdeten tropischen Waldtypen (Janzen, 1988) und sind dadurch ge- 
kennzeichnet, dass ein Teil der Bäume ihr Laub während der Trockenperiode abwirft, um sich vor dem Austrocknen zu schützen. Für die fernerkundliche Erfassung solcher Wälder spielt diese phänologische Besonderheit eine wichtige Rolle, da die Aufnahmen während der belaubten Phase stattfinden sollten und somit die mögliche Aufnahmezeit eingrenzen.
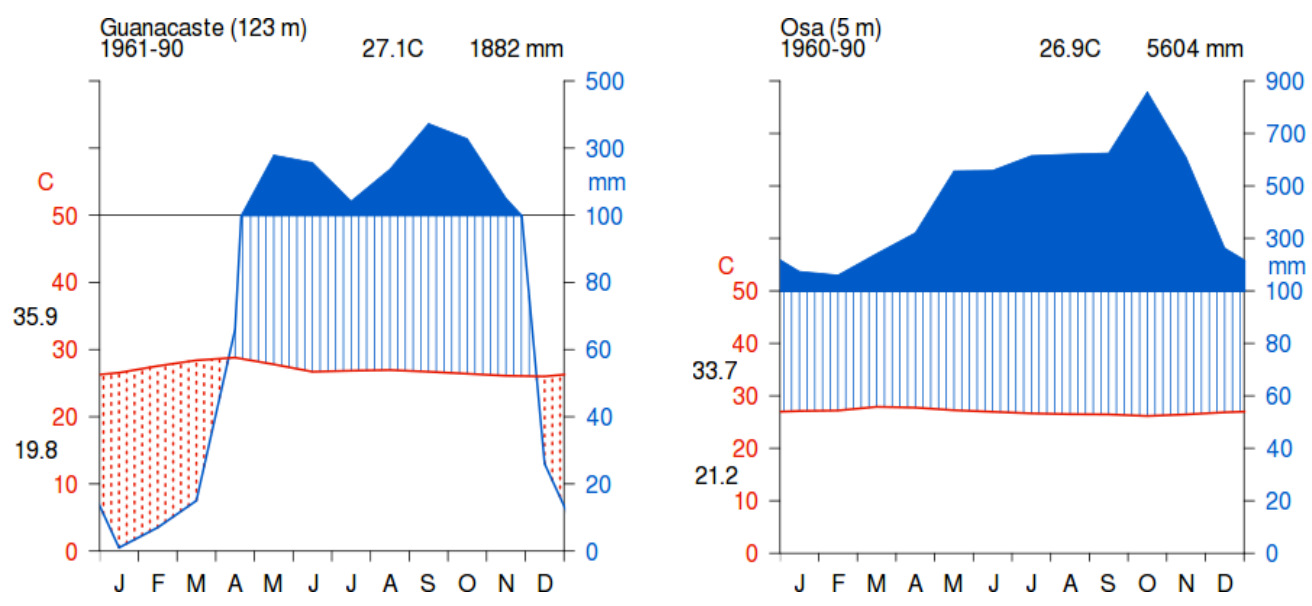

Abbildung 24.: Klimadiagramm nach Walter et al. (1964) für beide Untersuchungsgebiete. Die Klimadaten wurden aus der FAO-LocClim-Datenbank (Grieser et al., 2006) für die Mittelpunkte der Untersuchungsgebiete extrahiert: Osa: $-83.4^{\circ} \mathrm{W} ; 8.85^{\circ} \mathrm{N}$; Guanacaste: $85.37^{\circ} \mathrm{W} ; 10.1^{\circ} \mathrm{N}$.

\subsection{Bildmaterial}

\subsubsection{RapidEye}

Für die vorliegende Arbeit wurden Fernerkundungsdaten des RapidEye-Satellitensystems verwendet, das seit Februar 2009 kommerzielle Erdbeobachtungsdaten liefert. Dabei handelt es sich um eine Konstellation aus fünf baugleichen Satelliten, die sich in einer Höhe von $630 \mathrm{~km}$ in einem sonnen-synchronen Orbit befinden. Durch diese Konstellation kann für jeden Punkt der Erdoberfläche eine Aufnahme pro Tag erfolgen, wobei die Gesamtkapazität auf 4 Millionen $\mathrm{km}^{2}$ pro Tag beschränkt ist (RapidEye AG, 2012). Die verwendeten push-broom Sensoren des RapidEye-Systems zeichnen elektromagnetische Strahlung in fünf multispektralen Wellenlängenbereichen (siehe Tabelle 10) mit einer radiometrischen Auflösung von 12bit und einer räumlichen Auflösung von 6.5m auf. Die Daten werden in Form von 
zwei Produkttypen angeboten: als Basisdaten (Level 1B) und als orthorektifizierte Bilder (L3A). Für die vorliegende Arbeit wurden ausschließlich L3A Daten verwendet. Während der Orthorektifizierung werden die Bilddaten auf eine räumliche Auflösung von 5m interpoliert, als ganzahlige Werte mit 16bit abgespeichert und im GeoTIFF-Format ausgeliefert.

Tabelle 10.: Spektrale Auflösung der RapidEye-Sensoren (RapidEye AG, 2012)

\begin{tabular}{lll}
\hline Name & Band & Wellenlängen $(\mathrm{nm})$ \\
\hline Blau & 1 & $440-510$ \\
Grün & 2 & $520-590$ \\
Rot & 3 & $630-685$ \\
RedEdge & 4 & $690-730$ \\
NIR & 5 & $760-850$ \\
\hline
\end{tabular}

Beide Untersuchungsgebiete befinden sich in den Tropen, welche durch einen ganzjährig hohen Wasserdampfgehalt in der Atmosphäre ausgezeichnet sind. Bei der Auswahl der Aufnahmen für Osa wurde daher vor allem auf einen möglichst geringen Anteil an Wolken und Dunst geachtet. Für Guanacaste war es zusätzlich wichtig Aufnahmen von phänologisch günstigen Zeitpunkten zu verwenden. In Tabelle 11 sind alle Aufnahmen mit den dazugehörigen Aufnahmeparametern aufgelistet. Für Guanacaste wurden insgesamt neun Bilder am Ende der Regenzeit (Mitte November und Anfang Dezember) ausgewählt. Zu diesem Zeitpunkt tragen die Bäume noch ihre Blätter, aber die Bewölkung ist bereits zurückgegangen, so dass die Wahrscheinlichkeit, größere wolkenfreie Gebiete aufnehmen zu können, höher ist.

Die Bilder wurden auf Antrag (RESA Nr. 386) vom Deutschen Zentrum für Luft- und Raumfahrt (DLR) im Rahmen einer öffentlich-privaten Partnerschaft mit der RapidEye AG zur Verfügung gestellt. Nach Erhalt der Daten zeigte sich, dass der Bewölkungsgrad, wie er in den Metadaten angegeben ist, deutlich unterschätzt wurde. Zum Beispiel geben die Metadaten für das Bild O-01 1\% Bewölkung an. Der Vergleich mit dem oberen rechten Ausschnitt in Abbildung 32 zeigt jedoch eine deutlich höhere Bewölkung. Insofern war die Datenqualität niedriger als aus den Metadaten erwartet wurde.

Ein weiterer wichtiger Aspekt der Datenqualität von Fernerkundungsdaten ist die Lagegenauigkeit der Bilder. Diese wird für L3A Daten in Form des CE90-Wertes (Circular Error of 90\%) angegeben. Dieser Wert entspricht dem kleinsten Durchmesser des Kreises, der um alle Ground Controll Points (GCP) gelegt werden kann und in dem sich 90\% der da- 
Tabelle 11.: RapidEye-Aufnahmenparameter entnommen aus den mitgelieferten Metadaten mit CE90 = circular error $90 \%(\mathrm{~m})$

\begin{tabular}{lllllll}
\hline Bild ID & Region & Datum & Aufnahmezeit & Bewölkung & CE90 & Satellit \\
\hline O-01 & Osa & $2010-01-04$ & $16: 55: 01$ & $1 \%$ & 46.37 & RE-2 \\
O-02 & Osa & $2010-01-04$ & $16: 55: 04$ & $2 \%$ & 46.28 & RE-2 \\
O-03 & Osa & $2010-01-17$ & $16: 47: 44$ & $12 \%$ & 41.73 & RE-1 \\
O-04 & Osa & $2010-01-17$ & $16: 47: 48$ & $11 \%$ & 41.43 & RE-1 \\
\hline G-01 & Guanacaste & $2009-11-18$ & $17: 07: 48$ & $0 \%$ & 40.27 & RE-2 \\
G-02 & Guanacaste & $2009-11-18$ & $17: 07: 49$ & $0 \%$ & 39.41 & RE-2 \\
G-03 & Guanacaste & $2009-11-18$ & $17: 07: 52$ & $0 \%$ & 39.59 & RE-2 \\
G-04 & Guanacaste & $2009-11-20$ & $17: 07: 20$ & $12 \%$ & 46.10 & RE-4 \\
G-05 & Guanacaste & $2009-12-08$ & $17: 05: 48$ & $0 \%$ & 39.12 & RE-3 \\
G-06 & Guanacaste & $2009-12-08$ & $17: 05: 49$ & $0 \%$ & 38.16 & RE-3 \\
G-07 & Guanacaste & $2009-12-08$ & $17: 05: 52: 08$ & $0 \%$ & 38.35 & RE-3 \\
G-08 & Guanacaste & $2009-12-08$ & $17: 05: 52: 55$ & $0 \%$ & 39.35 & RE-3 \\
G-09 & Guanacaste & $2009-12-08$ & $17: 05: 53$ & $0 \%$ & 37.85 & RE-3 \\
\hline
\end{tabular}

zugehörigen Bildpunkte befinden. Der mittlere CE90-Wert betrug laut Metadaten $41.1 \mathrm{~m}$ (vergleiche Tabelle 11). Dabei ist jedoch zu beachten, dass die tatsächlichen Abweichungen zum Teil deutlich größer sein können, da die verwendeten GCP durch Interpretation von Landsat Bildern gewonnen werden. Die Lagegenauigkeit dieser Landsat-GCP wird mit einem CE90 $\leq 50 \mathrm{~m}$ angegeben (RapidEye AG, 2012). Die korrekte Lagegenauigkeit lässt sich somit nicht aus den Metadaten rekonstruieren und wurde im Feld mithilfe des Global Positioning System (GPS) erneut bestimmt.

\subsubsection{MODIS}

Für die Atmosphärenkorrektur wurden Informationen über die Zusammensetzung und den Zustand der Atmosphäre zum Zeitpunkt der Aufnahme mit Hilfe des MODIS-Sensors an Bord des AQUA-Satelliten gewonnen. Dieser Satellit ist seit 2002 im Orbit und wurde speziell für die Beobachtung der Wasserkreisläufe der Erde von der National Aeronautics and Space Administration (NASA) entwickelt. Der MODIS-Sensor zeichnet elektromagnetische Strahlung im sichtbaren und Infrarotbereich auf und speichert diese in 36 Kanälen ab. Aus diesen Kanälen wird eine Vielzahl von Produkten abgeleitet, welche sowohl für die Beobachtung der Land- und Wasseroberfläche als auch zur Beobachtung der atmosphärischen 
Zusammensetzung genutzt werden können. Eine ausführliche Darstellung der verschiedenen Produkte findet sich in Parkinson \& Greenstone (2000). In der vorliegenden Arbeit wurden für die Atmosphärenkorrektur die drei in Tabelle 12 gelisteten Produkte verwendet.

Tabelle 12.: Für die Atmosphärenkorrektur verwendete MODIS-Produkte.

\begin{tabular}{lllll}
\hline Produkt-ID & Band & Bandname & $\begin{array}{l}\text { räumliche } \\
\text { Auflösung }\end{array}$ & Bezeichnung \\
\hline MOD04 & 10 & Aerosol Optical Thickness & $10 \mathrm{~km}$ & AOT \\
MOD07 & 20 & Total Ozone & $5 \mathrm{~km}$ & OZONE \\
MOD07 & 24 & Water Vapor & $5 \mathrm{~km}$ & VAPOR \\
\hline
\end{tabular}

\subsubsection{SRTM}

Sowohl für die atmosphärische als auch für die topographische Korrektur der RapidEyeDaten wurden Informationen über die Topographie der Untersuchungsgebiete benötigt. Hierfür wurden Daten der Shuttle Radar Topography Mission (SRTM) verwendet. Die Aufnahmen wurden während des Fluges des NASA Space Shuttles Endeavour vom 11.-22. Februar 2000 mit einer Kombination aus X- und C-Band Radarantennen erstellt. Durch eine versetzte Montage beider Antennen mit einem Abstand von 60m konnte, durch eine interferometrische Analyse der Daten, ein Digitales Gelände Modell (DGM) mit einer räumlichen Auflösung von $90 \mathrm{~m}$ für alle Breitengrade von $56^{\circ}$ Süd bis $60^{\circ}$ Nord erstellt werden. Die vertikale Auflösung der SRTM-Daten ist nicht explizit beschrieben. Allerdings werden die Daten als ganzzahlige Werte (16bit) gespeichert und ausgeliefert, so dass die Auflösung $\geq 1 \mathrm{~m}$ sein muss. Für Osa und Guanacaste wurden die entsprechenden Rasterdaten zunächst mosaikiert und anschließend auf die Untersuchungsgebiete zugeschnitten und mit einem bikubischen Verfahren auf $5 \mathrm{~m}$ interpoliert. Anschließend wurde für jedes RapidEyePixel die Hangneigung und Exposition nach der Methode von Zevenbergen \& Thorne (1987) berechnet. 



\section{Erfassung der Referenzdaten}

\subsection{Trainings- und Validierungsdaten aus der Bildinterpretation}

Die Trainings- und Valididerungsdaten sind Grundlage der überwachten Klassifikation. Die Genauigkeit der Klassifikation als auch der Generalisierungsgrad der Ergebnisse hängt direkt von ihnen ab. Bedingt durch die verwendete Klassifikationsmethode ergeben sich verschiedene Anforderungen an die Auswahl und Art der Referenzdaten:

- randomisierte Auswahl

- Erfassung aller im Bild vorhandenen Klassen

- Erfassung der spektralen Varianz innerhalb der Klassen

- Erfassung der spektralen Varianz innerhalb des Bildes

Um den beschriebenen Anforderungen gerecht zu werden, wurde für jedes Untersuchungsgebiet ein systematisches Raster mit zufälligem Startpunkt angelegt, wobei die Abstände so gewählt wurden, dass ca. 50 Schnittpunkte innerhalb des Bildbereiches lagen. Bei der systematischen Stichprobe ist die Stichprobengröße eine Zufallsvariable, so dass die genaue Anzahl vorher nicht festgelegt werden kann. An jedem Schnittpunkt wurde eine Kartierfläche von $200 \times 200 \mathrm{~m}$ angelegt und nach dem Level-I Klassifikationsschlüssel von einem erfahrenen Bildinterpreten mit Ortskenntnis interpretiert.

Die Anforderungen an den Validierungsdatensatz entsprechen den oben aufgeführten Kriterien. Bei der Auswahl der Validierungsdaten ist im Besonderen darauf zu achten, dass sie unabhängig von den Trainingsgebieten erfolgt. Außerdem sollten die Validierungsdaten nicht für die Parametrisierung des Klassifikators verwendet werden. Da sie in der vorliegenden Arbeit ausschließlich für die Quantifizierung des Klassifikationsfehlers eingesetzt werden, können Punkte als Validierungseinheiten genutzt werden. Zur Auswahl der Validierungspunkte wurde auch ein systematisches Raster mit zufälligem Startpunkt verwendet, wobei die Rasterweite so gewählt wurde, dass ca. 100 Validierungspunkte in jedes Untersuchungsgebiet fielen. Da die Qualität der Karten in den Untersuchungsgebieten beurteilt werden sollte, erfolgte die Validierung auf Ebene der Untersuchungsgebiete. Daher wurden die Validierungspunkte über das gesamte Untersuchungsgebiet verteilt. Jeder Validierungspunkt 
wurde entsprechend des Level-I Klassifikationschlüssels interpretiert.

\subsection{Validierungsdaten aus terrestrischen Aufnahmen}

Die terrestrischen Aufnahmen verfolgte das Ziel, Validierungsdaten für die Kronenüberschirmung zu erheben. Aufgrund der gegebenen Zielsetzung war es wichtig sicherzustellen, dass der gesamte Wertebereich der vorkommenden Kronenüberschirmungen durch die Feldaufnahmen abgedeckt wird. Gleichzeitig standen nur begrenzte Ressourcen für die Feldaufnahmen zur Verfügung, so dass ein möglichst effizientes Stichprobenverfahren genutzt werden sollte. Unter diesen Voraussetzungen bieten sich stratifizierte Stichprobenverfahren an. Zur Stratifizierung wurde ein systematisches Raster mit zufälligem Startpunkt und einer Rasterweite von $200 \mathrm{~m}$ für beide Untersuchungsgebiete angelegt. Aufgrund der Untersuchungsgebietsgröße $(30 \mathrm{~km} \times 30 \mathrm{~km})$ ergaben sich 22500 Probeflächen.

Tabelle 13.: Geplante Verteilung der Probepunkte auf die vier Straten

\begin{tabular}{llll}
\hline Stratum & Kronenüberschirmung & Osa & Guanacaste \\
\hline 1 & $<0.25$ & 5 & 5 \\
2 & $\geq 0.25,<0.5$ & 5 & 5 \\
3 & $\geq 0.5,<0.75$ & 5 & 5 \\
4 & $\geq 0.75$ & 5 & 5 \\
\hline & Gesamt & 20 & 20 \\
\hline
\end{tabular}

Der potentielle Wertebereich der Zielgröße Kronenüberschirmung ist $0 \leq x \leq 1$. Um diesen Wertebereich in der Stichprobe abzudecken, wurden die Probeflächen in $L=4$ Straten aufgeteilt (siehe Tabelle 13). Die jeweilige Überschirmungsklasse wurde durch Verschnitt des systematischen Rasters mit den klassifizierten RapidEye-Bildern bestimmt. Anschließend wurden aus jedem Stratum zufällig fünf Probeflächen ausgewählt und im Feld aufgesucht. Diese Anzahl von $n=20$ Probeflächen pro Untersuchungsgebiet ergab sich aufgrund der limitierten Mittel und des hohen Aufwandes der Feldarbeiten. Sie stellt somit einen Kompromiss zwischen Genauigkeit und den zur Verfügung stehenden Ressourcen dar. 


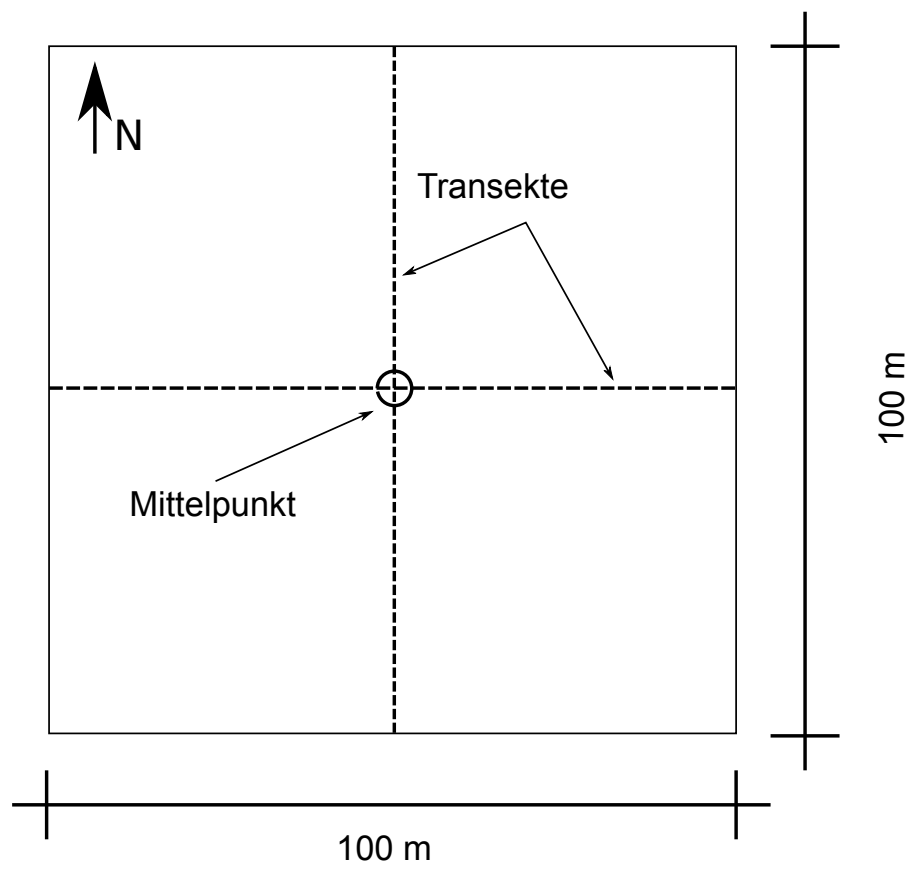

Abbildung 25.: Probeflächendesign der terrestrischen Aufnahmen

Die Erfassung der Kronenüberschirmung im Rahmen von stichprobenbasierten Waldinventuren ist aufwendig und wird nur selten durchgeführt. Trotzdem existieren verschiedene Methoden. $\mathrm{Zu}$ den am häufigsten verwendeten Methoden gehören das Linienabschnittsverfahren (Gregoire \& Valentine, 2008; Ko et al., 2009), systematische Punktstichproben (Korhonen et al., 2006), Densitometer Messungen (Lemmon, 1956) und die Kronenkartierungen mit Hilfe von Kronenspiegeln. Die Punktstichproben sind sehr zeitaufwendig, da für jede Probefläche ca 200-250 Messungen erforderlich sind (Korhonen et al., 2006). Aus praktischen Gründen wurde daher das Linienabschnittsverfahren gewählt. Das Probeflächendesign bestand, wie in Abbildung 25 dargestellt, aus zwei Transekten mit einer Länge von je $100 \mathrm{~m}$. Im Feld wurden diese Transekte mit Hilfe von zwei 50m Rollmaßbändern abgesteckt. Die Aufnahmen erfolgten in der Regel in einem Team aus drei Personen. Nach dem Auffinden des Stichprobenpunktes mit Hilfe eines GPS-Gerätes und einer für jeden Punkt angefertigten Feldkarte (siehe Abbildung 36 im Anhang) wurde von zwei Personen die Transekte angelegt. Dazu stellte sich die erste Person auf den Stichprobenmittelpunkt. Die zweite Person wurde dann mittels Bussole eingewiesen, so dass die Rollmaßbänder in die entsprechende Richtung ausgelegt werden konnten. Sobald ein Transekt fertig ausgelegt war, konnte die dritte Person mit dem Messen der Kronenenabschnitte beginnen. Die Startund Endpunkte der von Baumkronen überschirmten Abschnitte des Transektes wurden mit 
Hilfe eines Kronenspiegels bestimmt und im Aufnahmebogen notiert. An Punkten mit dichten Unterstand war es notwendig, die Pflanzen entlang der Transekte zu entfernen, um das Rollmaßband auslegen zu können. Aus praktischen Gründen konnte dies jedoch nur bis zu einer Höhe von ca. $2 \mathrm{~m}$ erfolgen. Bei der Aufnahme mit dem Kronenspiegel wurde jedoch versucht Pflanzenteile, die niedriger als $5 \mathrm{~m}$ waren, nicht zu berücksichtigen. 


\section{Ergebnisse der Fallstudie}

\subsection{Bildkalibrierung}

\subsubsection{Atmosphärenkorrektur}

Der Zustand und die Zusammensetzung der Atmosphäre unterliegen starken wetter- und tageszeitabhängigen Schwankungen. Für die atmosphärische Korrektur war es daher wichtig eine möglichst geringe zeitliche Differenz zwischen den MODIS- und den RapidEyeAufnahmen sicherzustellen. Die RapidEye-Aufnahmen in Osa erfolgten an zwei Tagen, für die jeweils geeignete MODIS-Produkte mit geringen zeitlichen Differenz gefunden werden konnten (vergl. Tabelle 14). In Guanacaste erfolgten an drei Tagen RapidEye-Aufnahmen. In den korrespondierenden MODIS-Aufnahme waren jedoch große Bildbereiche von Wolken überdeckt, so dass nicht für alle RapidEye-Aufnahmen geeignete Werte aus den MODISProdukten extrahiert werden konnten. In den Fällen, für die keine Informationen vorlagen, wurde der Mittelwert für die Parametrisierung des 6S-Modells verwendet. Da beide Untersuchungsgebiete an den Pazifik angrenzen, wurde ein maritimes Aerosolmodell gewählt, wobei der AOT-Wert ebenfalls aus den MODIS-Daten berechnet wurde.

Vergleicht man die aus den MODIS-Daten abgeleiteten Atmosphärenparameter in Tabelle 14 so wird klar, dass die Zusammensetzung der Atmosphäre während der verschiedenen Aufnahmetage unterschiedlich war. Beispielsweise wurden in Osa am 17. Januar 2010 höhere Wasserdampf-, Ozon- und Aerosolmengen gemessen als am 4. Januar 2010. Für die kombinierte Analyse der Bilder mit unterschiedlichem Aufnahmedatum wurde daher eine atmosphärische Korrektur der Rohdaten mit dem 6S-Modell und den in Tabelle 14 angegebenen Atmosphärenparametern durchgeführt. Durch die Anwendung des Modells wurden die Grauwerte der Rohdaten in die physikalische Größe $\rho_{s}$, den Anteil der Bodenreflexion, überführt. Da es sich dabei um ein Anteilswert handelt, ergibt sich für $\rho_{s}$ ein Wertebereich von $0-1$.

Der Vergleich der Dichtefunktionen in Abbildung 26 zeigt Unterschiede in der Verteilung der Pixelwerte vor und nach der Atmosphärenkorrektur. Durch die Anwendung des 6SModells werden sowohl die Mittelwerte als auch die Streuung der Pixelwerte verändert. Am 
Tabelle 14.: Aus den MODIS-Daten berechnete Atmosphärenparameter mit: VA$\mathrm{POR}=$ Total Column Precipitable Water Vapor-IR Retrieval, OZONE=Total Ozone Burden, AOT=Aerosol optical thickness at 550nm. Klammern zeigen an, dass keine Messungen vorlagen und daher der jeweilige Mittelwert verwendet wurde.

\begin{tabular}{llllll}
\hline Bild-ID & Datum & Aufnahmezeit & AOT & OZONE $[\mathrm{cm}-\mathrm{atm}]$ & VAPOR $\left[\mathrm{g} / \mathrm{m}^{2}\right]$ \\
\hline O-01 & $2010-01-04$ & $16: 30$ & 0.042 & 0.258 & 2.432 \\
O-02 & $2010-01-04$ & $16: 30$ & 0.053 & 0.256 & 2.705 \\
O-03 & $2010-01-17$ & $15: 55$ & 0.065 & 0.261 & 3.159 \\
O-04 & $2010-01-17$ & $15: 55$ & 0.066 & 0.260 & 3.407 \\
\hline G-01 & $2009-11-18$ & $15: 35$ & 0.138 & $(0.254)$ & $(4.325)$ \\
G-02 & $2009-11-18$ & $15: 35$ & $(0.053)$ & $(0.254)$ & $(4.325)$ \\
G-03 & $2009-11-18$ & $15: 35$ & $(0.053)$ & $(0.254)$ & $(4.325)$ \\
G-04 & $2009-11-20$ & $17: 00$ & $(0.053)$ & $(0.254)$ & $(4.325)$ \\
G-05 & $2009-12-08$ & $16: 45$ & 0.041 & 0.256 & 4.725 \\
G-06 & $2009-12-08$ & $16: 45$ & 0.027 & 0.259 & 4.666 \\
G-07 & $2009-12-08$ & $16: 45$ & $(0.053)$ & 0.246 & 3.930 \\
G-08 & $2009-12-08$ & $16: 45$ & 0.031 & 0.252 & 4.134 \\
G-09 & $2009-12-08$ & $16: 45$ & 0.029 & 0.255 & 4.145 \\
\hline
\end{tabular}

deutlichsten ist das für das blaue Band zu beobachten, welches durch die Korrektur nach links verschoben wird. Das heißt, dass das 6S-Modell die Strahlungsanteile im Wellenlängenbereich von 440-510nm (blau) reduziert. Gerade in dem kurzwelligen Bereich kommt es aufgrund der Streuung an den Wassermolekühlen zu einer Überschätzung der Reflexion am Boden, welche durch die Korrektur reduziert wird. Im Gegensatz dazu wird der Anteil der Strahlung im NIR-Bereich erhöht, was an einer Verschiebung der Dichtefunktion nach rechts zu erkennen ist. Die Reflexion im NIR-Bereich ist am höchsten. Neben der Verschiebung ändert sich auch die Streuung: Im korrigierten Bild kann eine stärkere Streuung beobachtet werden.

Die Analyse der Bildstatistiken ergab Pixelwerte außerhalb des plausiblen Wertebereiches. Dies deutet daraufhin, dass das 6S-Modell den Strahlungsdurchgang durch die Atmosphäre während der RapidEye-Aufnahmen nicht für alle Pixel korrekt abbildet. Dies kann durch Differenz zwischen den zur Anpassung des 6S-Modells verwendeten Atmosphärenparametern und der tatsächlichen während der RapidEye-Aufnahmen gegebenen Zusammensetzung der Atmosphäre verursacht werden. Solche Unterschiede können entweder auf zeitlichen oder räumlichen Differenzen zwischen den MODIS- und den RapidEye-Aufnahmen beruhen. Die zeitliche Differenz beträgt für die Aufnahmen in Osa weniger als 2h (vergl. Tabelle 14 

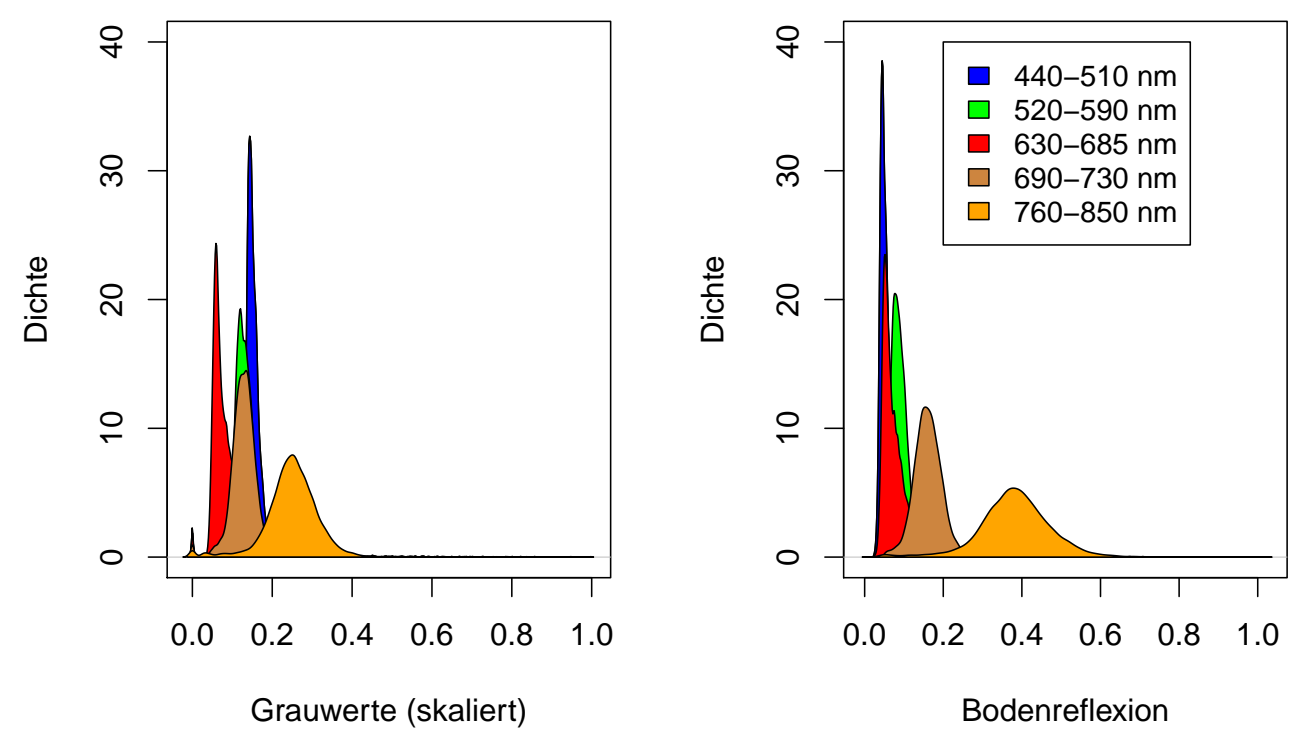

Abbildung 26.: Mit dem Kerndichteschätzer nach Sheather \& Jones (1991) angepasste Dichtefunktion der Pixelwerte des Bildes O-01 vor (links) und nach (rechts) der atmosphärischen Korrektur für die fünf RapidEye-Bänder.

und 11), aber besonders in den Nachmittagsstunden, in welchen alle RapidEye-Aufnahmen erfolgten, kann sich der Zustand der Atmosphäre sehr schnell ändern. In Guanacaste standen nicht für alle Aufnahmetage geeignete MODIS-Aufnahmen zur Verfügung. In diesen Fällen wurde der Mittelwert verwendet, was zwangsläufig zu einer Differenz zwischen modellierter und tatsächlicher Atmosphäre führt. Räumliche Differenzen können bei kleinräumiger Variation der Atmosphärenbedingungen auftreten. Da die verwendeten MODIS-Produkte eine räumliche Auflösung von 5 bzw. 10km haben, können räumliche Variationen $<5 \mathrm{~km}$ nicht abgebildet werden.

Abbildung 27 zeigt Spektralprofile über einem Transekt im Überlappungsbereich zweier RapidEye-Aufnahmen. Unterstellt man eine konstante Kalibrierung des Sensors und dass es in den 13 Tagen zwischen beiden Aufnahmen keine wesentlichen Änderungen der Landbedeckung gegeben hat, müssen die Differenzen beider Profile auf Unterschiede in der Atmosphäre zurückgeführt werden. Im Idealfall sollte die atmosphärische Korrektur diese Differenzen vollständig ausgleichen. Die kalibrierten Profile in Abbildung 27 (rechts) zeigen 

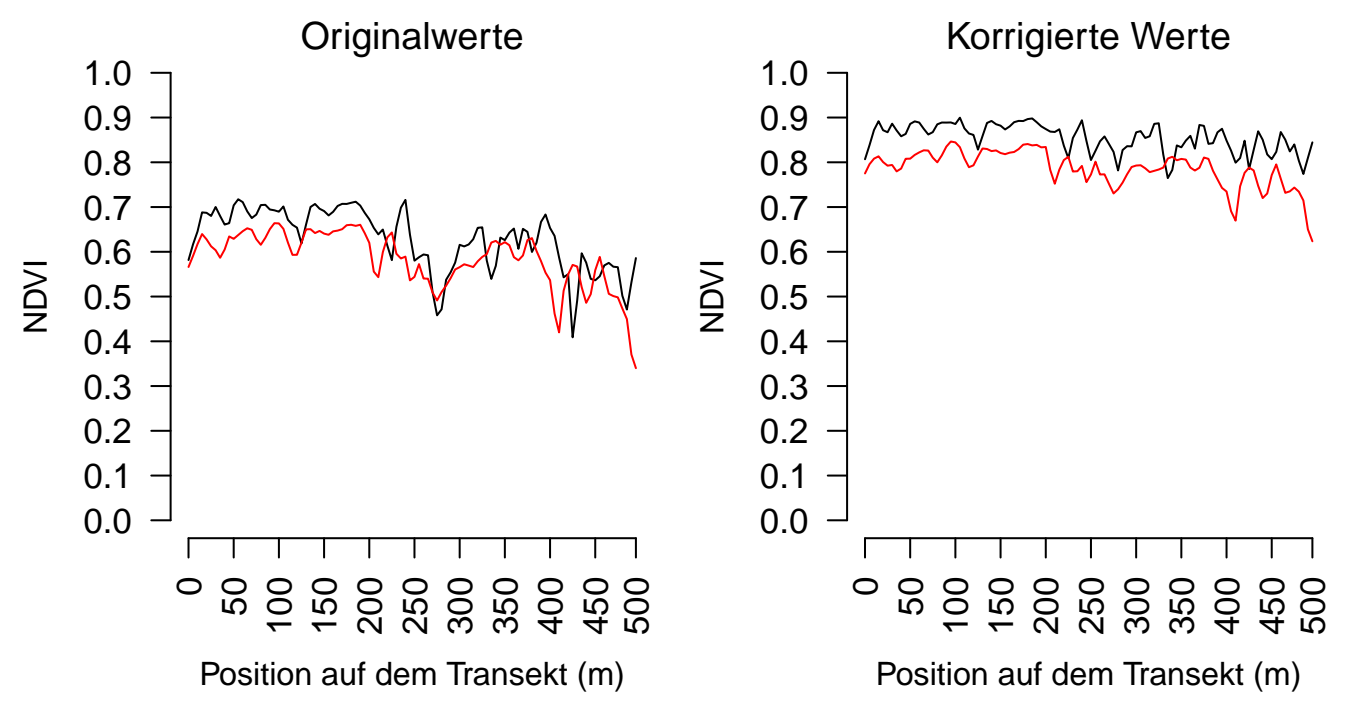

Abbildung 27.: NDVI-Werte entlang eines 500m langen Transektes im Überlappungsbereich der RapidEye-Aufnahmen vom 17.1.2010 (schwarz) und vom 04.01.2010 (rot) berechnet aus den originalen Bildern (links) und aus den atmosphärisch korrigierten Bildern (rechts).

jedoch auch nach der Kalibrierung noch Differenzen zwischen beiden Aufnahmen.

\subsubsection{Georeferenzierung}

Das stratifizierte Stichprobendesign basierte auf der Auswahl von Stichprobenpunkten in Abhängigkeit ihrer aus dem RapidEye-Bild bestimmten Kronenüberschirmung. Daher war es notwendig, dass die Feldaufnahmen exakt an den im Bild identifizierten Punkten erfolgten. Während der Feldaufnahmen zeigte sich, dass die Lagegenauigkeit der RapidEye-Bilder teilweise noch geringer als in den Metadaten angegeben war. Der Lageversatz der RapidEyeAufnahmen führte dazu, dass die im Feld per GPS aufgesuchten Stichprobenpunkte nicht mit der Position in der Karte übereinstimmte, wodurch die Kronenüberschirmung nicht mehr dem angestrebten Stratum entsprach. Daher wurden die Positionen anhand von markanten Anhaltspunkten, z. B. Straßenkreuzungen, Wege, Einzelbäume, Wasserfläche oder Stromtrassen, die im Feld und im RapidEye-Bild eindeutig bestimmt werden konnten, neu ermittelt. Dabei erwiesen sich die Feldkarten, die für jeden Stichprobenpunkt angelegt wurden, als sehr hilfreich (siehe Abbildung 36). Schwierig war die Identifizierung der Punkte in der höchsten Überschirmungsklasse, da diese sich im dichten Wald befanden, wo keine mar- 
kanten Punkte in Satellitenbildern identifiziert werden können. Hier konnten die vorher im Bild bestimmten Punkte nicht immer eindeutig erkannt werden, was aber keinen Einfluss auf die Stratenzuordnung hatte. Auf allen Punkten erfolgte für die Dauer der Aufnahme eine Langzeitmessung mit dem GPS, wodurch die korrigierte Position der Probekreismittelpunkte mit einem Lagefehler $\leq 5 \mathrm{~m}$ bestimmt werden konnte.

Während der Feldaufnahmen wurden in beiden Untersuchungsgebieten bei den An- und Abfahrten zu den Stichprobenpunkten Bodenkontrollpunkte eingemessen. Dafür wurden die Koordinaten markanter Punkte, wie Straßenkreuzungen, Gebäudeecken und Brücken, die sich im RapidEye-Bild eindeutig lokalisieren lassen, mit dem GPS-Gerät gemessen. Nach Abschluss der Feldaufnahmen erfolgte eine erneute Georeferenzierung der RapidEye-Bilder mit den gemessenen Bodenkontrollpunkten. Für die Bildbereiche außerhalb der Untersuchungsgebiete, für die keine Bodenkontrollpunkte vorlagen, wurden Bildverknüpfungspunkte verwendet. Die so durchgeführte Georeferenzierung ergab einen mittleren Lagefehler von $<$ 10m (siehe Tabelle 15). Im Vergleich zu den ursprünglichen Lagefehlern in den Originaldaten (vergl. Tabelle 11) konnte die Lagegenauigkeit also deutlich verbessert werden.

Tabelle 15.: Kontrollpunktanzahl und mittlere Lagefehler der Georeferenzierung der RapidEye-Daten bezogen auf die Bodenkontrollpunkte.

\begin{tabular}{llll}
\hline Bild ID & Region & Kontrollpunkte & Lagefehler \\
\hline O-01 & Osa & 16 & $6.77 \mathrm{~m}$ \\
O-02 & Osa & 12 & $14.79 \mathrm{~m}$ \\
O-03 & Osa & 04 & $9.42 \mathrm{~m}$ \\
O-04 & Osa & 04 & $4.73 \mathrm{~m}$ \\
\hline G-01 & Guanacaste & 08 & $9.30 \mathrm{~m}$ \\
G-02 & Guanacaste & 09 & $19.88 \mathrm{~m}$ \\
G-03 & Guanacaste & 08 & $7.01 \mathrm{~m}$ \\
G-04 & Guanacaste & 05 & $5.06 \mathrm{~m}$ \\
G-05 & Guanacaste & 09 & $8.59 \mathrm{~m}$ \\
G-06 & Guanacaste & 23 & $2.12 \mathrm{~m}$ \\
G-07 & Guanacaste & 05 & $10.27 \mathrm{~m}$ \\
G-08 & Guanacaste & 12 & $6.44 \mathrm{~m}$ \\
G-09 & Guanacaste & 04 & $4.93 \mathrm{~m}$ \\
\hline & Mittelwert: & 9.2 & $8.41 \mathrm{~m}$ \\
\hline
\end{tabular}




\subsubsection{Korrektur der reliefbedingten Beleuchtungsunterschiede}

Durch die Aufnahmezeit am späten Nachmittag und dem damit verbundenen niedrigen Sonnenstand zeigten sich in den stark reliefierten Teilen der Untersuchungsgebiete deutliche Unterschiede zwischen Schatten- und Sonnenhängen. Zur Reduktion dieser reliefbedingten Beleuchtungsunterschiede erfolgte eine topographische Korrektur.

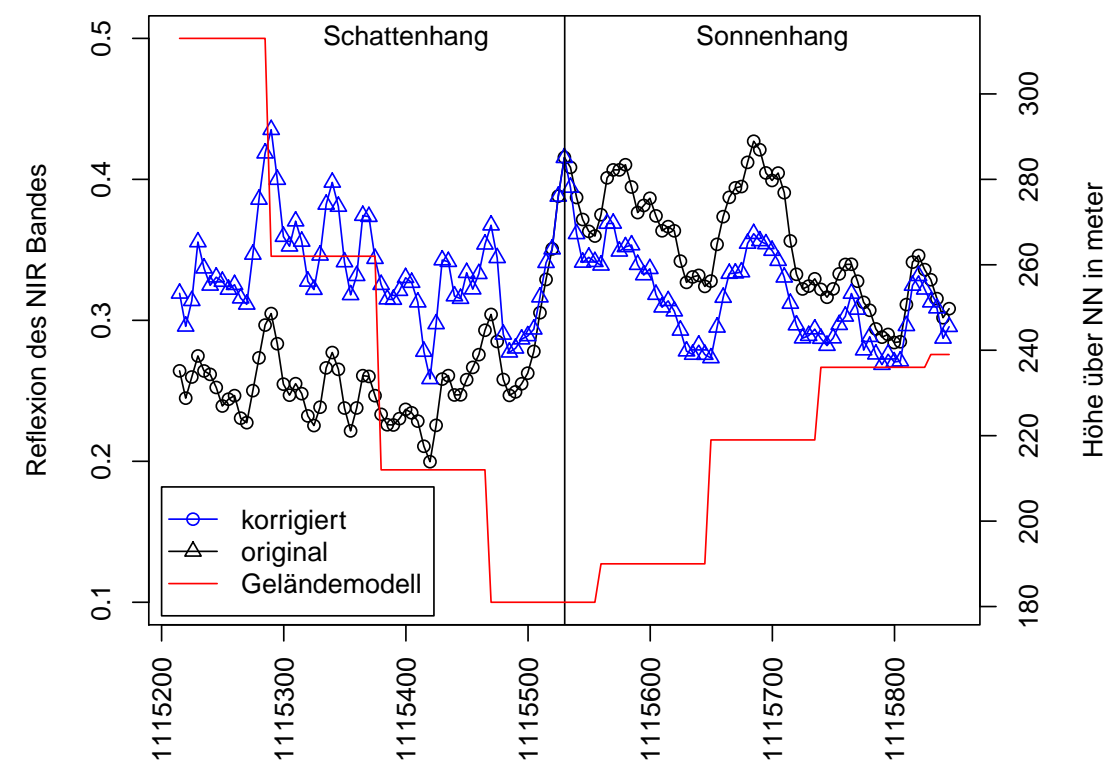

Abbildung 28.: Vergleich der Reflexionswerte des NIR-Bandes vor und nach der topographischen Korrektur entlang eines Transektes von einem Schatten- zu einem Sonnenhang innerhalb ein und derselben Landbedeckungsklasse.

Abbildung 28 zeigt den Effekt der topographischen Korrektur beispielhaft entlang eines Transektes. Vor der Korrektur (dunkle Linie) zeigt sich das erwartete Muster mit systematisch niedrigeren Reflexionswerten für den Schattenhang und höheren für den Sonnenhang. Die topographische Korrektur (helle Linie) reduziert diese Unterschiede deutlich. Der Schnittpunkt beider Profile befindet sich exakt in der Talsohle. Links und rechts des Schnittpunktes verlaufen beide Kurven annähernd parallel, was darauf hin deutet, dass die Variationen der Reflexion entlang des Transektes, die nicht durch die Topographie sondern durch die Reflexionseigenschaften der Landbedeckung beeinflusst wird, erhalten bleiben.

Die visuelle Kontrolle der korrigierten Bilder in Guanacaste zeigte, dass die Beleuchtungs- 
unterschiede nur in dem zentralen Bild (G-06) reduziert werden konnten, was auf die Genauigkeit der Georeferenzierung zurückgeführt werden kann. Für das zentrale Bild standen $n=23$ Kontrollpunkte zur Verfügung, mit denen eine Lagegenauigkeit von $2.12 \mathrm{~m}$ erreicht wurde (vergl. Tabelle 15). Die übrigen Bilder wurden nur mit Hilfe von Verknüpfungpunkten an das zentrale Bild angepasst, so dass die Lagegenauigkeit in den äußeren Bereichen deutlich niedriger ist. Eine Koregisitrierung der SRTM- und RapidEye-Daten war nicht möglich, da im Untersuchungsgebiet keine markanten Punkte vorhanden sind, die eindeutig im Geländemodell identifiziert werden können. Die Lagegenauigkeit der SRTM-Daten wird für Südamerika mit 9m (CE90) angegeben (Rodriguéz et al., 2006). Daher muss davon ausgegangen werden, dass die Differenzen zwischen Bild und Geländemodell in den äußeren Bildern aufgrund der fehlenden GCP $>50 \mathrm{~m}$ sein können, was die Beleuchtungskorrektur stark einschränkt.

\subsection{Analyse der RF-Modelle}

\subsubsection{Parametrisierung der RF-Modelle}

Bevor die RF-Modelle für die Level-I Klassifikation auf Basis der Trainingsdaten trainiert werden konnten, mussten zunächst Werte für drei Parameter festgelegt werden:

Anzahl der Entscheidungsbäume Ein wichtiger Parameter für die Erstellung eines RFModells ist die Anzahl der in dem Modell enthaltenen Entscheidungsbäume $n$. Diese beeinflusst sowohl die Klassifikationsgenauigkeit als auch die Berechnungszeiten, welche sich proportional zu der Anzahl der Bäume erhöhen. Daher muss ein Kompromiss zwischen Genauigkeit und Berechnungsaufwand gefunden werden. Um zu ermitteln, welche Anzahl einen geeigneten Kompromiss darstellt, wurden $\mathrm{RF}$-Modelle mit $n=(1,21, \ldots, 501)$ Entscheidungsbäumen erzeugt und der Testfehler mit Hilfe einer 10-fachen Kreuzvalidierung bestimmt. Abbildung 29 (links) zeigt den Kreuzvalidierungsfehler in Abhängikeit der Anzahl $n$. Mit zunehmender Anzahl nimmt der Fehler und die Steigung der Kurve ab, so dass für RF-Modelle mit einer Anzahl > 100 nur noch eine geringe Verbesserung festgestellt werden kann. Für die Level-I Klassifikation wurde daher der Wert $n=100$ Entscheidungsbäume festgelegt. 

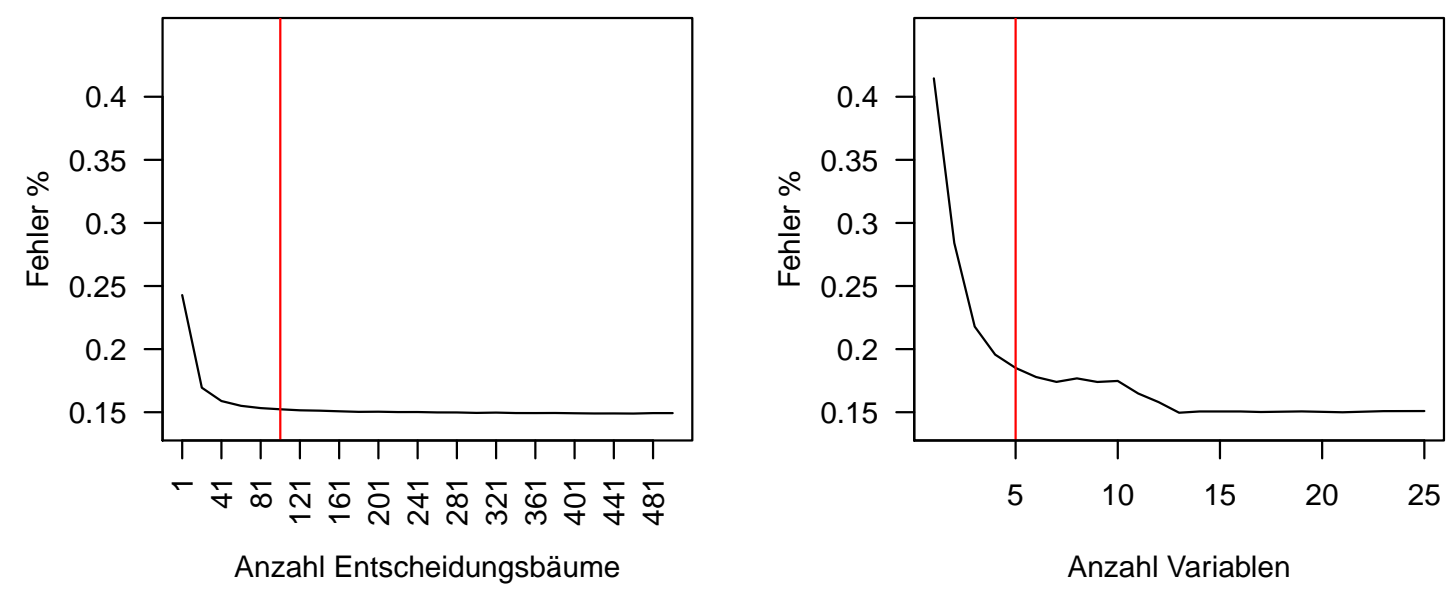

Abbildung 29.: Modellfehler für das Bild O-01. Links: für unterschiedliche Anzahlen von Entscheidungsbäumen, rechts: für unterschiedliche Anzahl von Variablen bei konstanter Anzahl von Entscheidungsbäumen $n=100$. Die senkrechten Linien markieren die gewählten Werte für beide Parameter.

Anzahl der Variablen im RF-Modell Die Anzahl der Variablen $M$, die beim Training des RF-Modells berücksichtigt werden, beeinflusst im wesentlichen die Komplexität der Modelle. Um die Modelle so einfach wie möglich zu halten, sollte das Modell mit der geringsten Anzahl an Variablen, das die Genauigkeitsanforderungen erfüllt, gewählt werden. Um zu untersuchen, wie der Fehler durch die Anzahl an Variablen beeinflusst wird, wurden RFModelle für $M=(1,2, \ldots, 25)$ erstellt und ebenfalls mit einer 10-fachen Kreuzvalidierung der Modellfehler bestimmt. Wie bei der Anzahl der Bäume ist in Abbildung 29 klar zu erkennen, dass mit zunehmender Anzahl $M$ der Fehler und die Steigung der Kurve abnimmt. Als Kompromiss zwischen möglichst hoher Genauigkeit und geringer Komplexität wurde ein Wert von $M=5$ Variablen festgelegt.

Anzahl der Variablen pro Entscheidungsknoten Um die Korrelation zwischen den Entscheidungsbäumen innerhalb eines RF-Modells zu minimieren, entwickelte Breiman (2001) für die RF-Modelle die so genannte „Sub-Space“-Methode. Dabei werden an jedem Entscheidungsknoten innerhalb eines Baums nicht alle $M$ Variablen berücksichtigt, sondern $m<M$ Variablen zufällig ausgewählt. In der Orignalfassung des RF-Programms von Brei- 
man \& Cutler (2012) wird $m=\sqrt{M}$ als Standardwert vorgeschlagen. Dieser Wert wurde auch hier verwendet.

Um die verschiedenen RF-Modelle besser vergleichen zu können, wurden die festgelegten Parameterwerte für alle Bilder in beiden Untersuchungsgebieten verwendet.

\subsubsection{Variablenselektion}

Insgesamt standen für die Klassifikation 25 Variablen zur Verfügung (5 Spektralbänder + 6 Vegetantionsindices +14 Texturindices) von denen die $M=5$ Bänder mit den höchsten Z-Scores gewählt wurden. Abbildung 30 zeigt die Z-Scores der Bänder aus den vier Bildern des Untersuchungsgebietes Osa.

Interessant ist, dass unter den fünf Bändern mit den höchsten Z-Scores bei allen Bildern sowohl originale Bänder als auch Textur- und Vegetationsindices zu finden sind. Offensichtlich ist die Kombination aus spektralen Bändern, Vegetations- und Texturindices besonders geeignet, die Level-I Landbedeckungsklassen zu unterscheiden, da sie jeweils unterschiedliche physiologische Aspekte der Vegetation erfassen. Auffällig ist, dass kein Texturindex zweiter Ordnung unter den fünf Bändern mit den höchsten Z-Scores ist. Die Rangfolge der Bänder nach den Z-Scores ist in allen Bildern unterschiedlich, was auf die verschiedenen Landbedeckungsmuster in den jeweiligen Bildern zurückgeführt werden kann. Daher wurden im Gegensatz zur Auswahl der RF-Parameter bei der Variablenselektion für jedes Bild die fünf Bänder mit den höchsten Z-Scores gewählt.

\subsubsection{Güte der RF-Modelle}

Die Güte der erstellten RF-Modelle ist mit einem mittleren Kappawert von 0.84 für $M=25$ Bänder als hoch einzustufen. Dabei unterscheiden sich die Qualität der einzelnen Modelle um bis zu 10\% der Gesamtgenauigkeit, was auf die unterschiedliche Bildqualität, die Ausprägung der Landbedeckung und die Trainingsdaten zurückzuführen ist. Durch die systematische Auswahl der Trainingsgebiete wurden nicht in jedem Bild alle Klassen erfasst, so dass die Anzahl der Klassen zwischen den Modellen variiert. Der Vergleich zwischen den Modellen mit $M=25$ und $M=5$ Bändern ergibt für alle Bilder eine Reduktion der Modellgüte um weniger als 5\%. Die Reduktion der Anzahl der Bänder führt zu einer starken Vereinfachung der Modelle, so dass die Gefahr einer Überanpassung reduziert werden kann. 

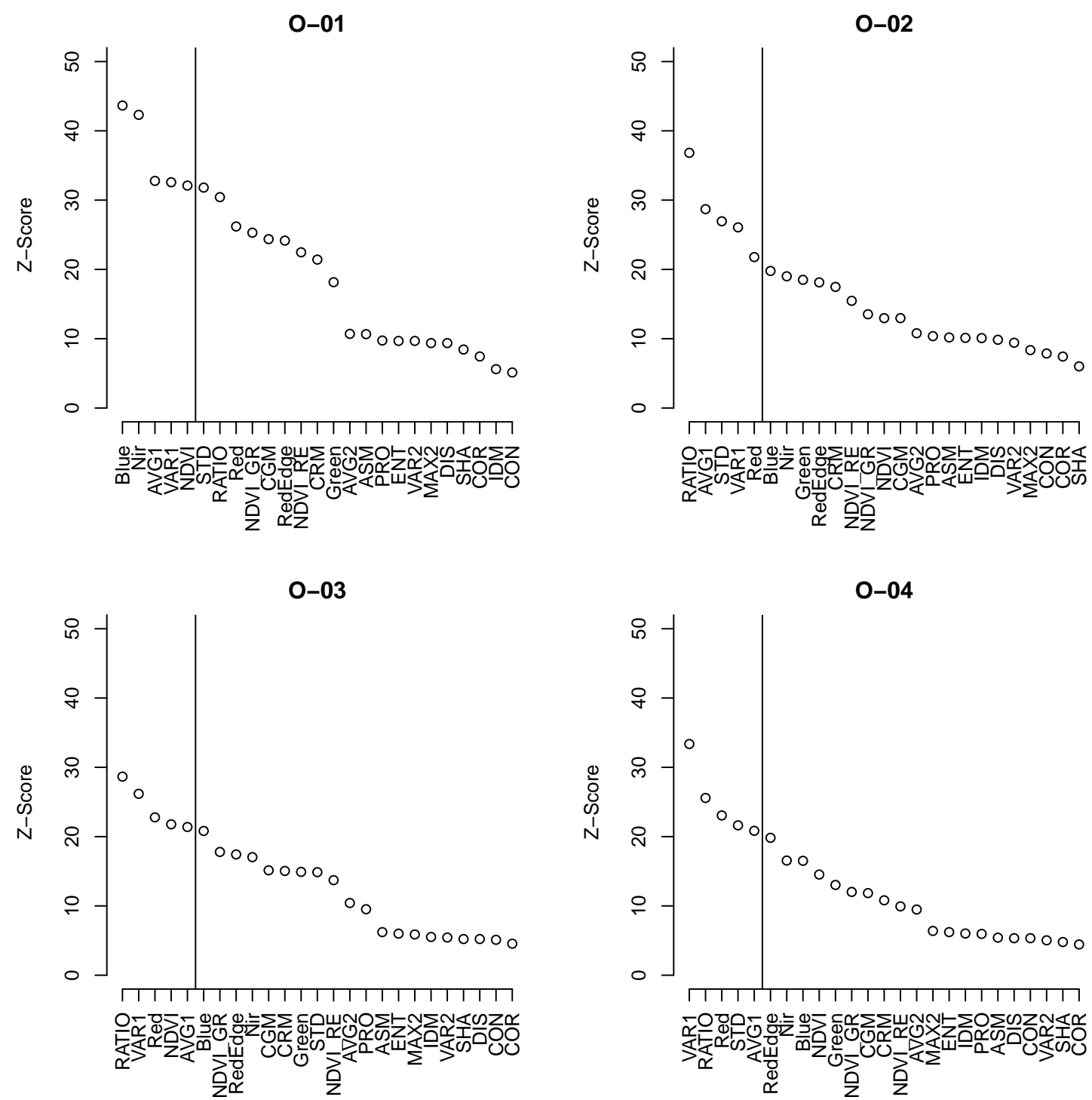

Abbildung 30.: Z-Scores der Bilder O-01 - O-04 (siehe Tabelle 11) für alle 25 Bänder berechnet aus den RF-Modellen (siehe Tabelle 16). Eine Erläuterung der Bandnamen findet sich in den Tabellen 7, 8 und 9. Die senkrechte Linie markiert die fünf Bänder mit den höchsten Z-Scores, welche für die Klassifikation verwendet wurden. 
Außerdem bietet die Reduktion den Vorteil, dass die Entscheidungsbäume innerhalb des Modells noch sinnvoll interpretierbar bleiben und die Berechnungszeiten drastisch reduziert werden können. Daher wurde für die weitere Klassifizierung mit den reduzierten Modellen gearbeitet und der geringe Verlust an Genauigkeit akzeptiert.

Tabelle 16.: Modellfehler der RF-Modelle mit $n=100$ Bäumen mit $M=25$ : allen berechneten Bändern und und $M=5$ : den fünf Bändern mit den höchsten Z-Scores

\begin{tabular}{|c|c|c|c|c|c|}
\hline \multirow[t]{2}{*}{ Bild ID } & \multirow[t]{2}{*}{ Anzahl Klassen } & \multicolumn{2}{|c|}{ Kappa } & \multicolumn{2}{|c|}{ OOB-Fehler } \\
\hline & & $M=25$ & $M=5$ & $M=25$ & $M=5$ \\
\hline O-01 & 11 & 0.81 & 0.66 & $8.95 \%$ & $15.59 \%$ \\
\hline O-02 & 11 & 0.92 & 0.9 & $5.73 \%$ & $6.95 \%$ \\
\hline $\mathrm{O}-03$ & 10 & 0.92 & 0.9 & $5.37 \%$ & $6.95 \%$ \\
\hline $\mathrm{O}-04$ & 10 & 0.88 & 0.79 & $7.51 \%$ & $13.29 \%$ \\
\hline G-01 & 9 & 0.89 & 0.81 & $6.3 \%$ & $11.7 \%$ \\
\hline G-02 & 9 & 0.77 & 0.65 & $16.4 \%$ & $24.8 \%$ \\
\hline G-03 & 10 & 0.58 & 0.51 & $12.68 \%$ & $14.8 \%$ \\
\hline G-04 & 9 & 0.88 & 0.84 & $7.39 \%$ & $11.2 \%$ \\
\hline G-05 & 7 & 0.98 & 0.93 & $0.72 \%$ & $3.2 \%$ \\
\hline G-06 & 8 & 0.81 & 0.7 & $8.00 \%$ & $12.7 \%$ \\
\hline G-07 & 9 & 0.95 & 0.91 & $3.38 \%$ & $5.8 \%$ \\
\hline G-08 & 10 & 0.63 & 0.52 & $16.47 \%$ & $21.6 \%$ \\
\hline \multirow[t]{2}{*}{ G-09 } & 6 & 0.99 & 0.98 & $0.5 \%$ & $0.8 \%$ \\
\hline & Mittelwert: & 0.832 & 0.762 & $8.39 \%$ & $12.18 \%$ \\
\hline
\end{tabular}

\subsection{Evaluation der Landbedeckungskarten}

Das entwickelte Klassifikationsverfahren beruht auf einer hierarchischen Klassifikation, wobei Landkarten mit drei thematischen Aufösungen erzeugt wurden. Aufgrund der hierarchischen Struktur hängen die Klassifikationsergebnisse direkt voneinander ab. Das heißt, dass die Klassifikationsfehler von einer Ebene auf die höheren Ebenen vererbt werden. Daher wurde die Validierung zunächst auf die unterste Ebene (Level-I) beschränkt. Durch die Abnahme der Klassenzahl von Level-I $(n=14)$ über Level-II $(n=5)$ zu Level-III $(n=3)$ nimmt die Wahrscheinlichkeit von Verwechslungen zwischen den Klassen und somit auch der Klassifikationsfehler ab. Ein Effekt, der z. B. von Walker et al. (2010) für Waldflächenkartierungen im Amazonasbecken beschrieben wurde. Insofern kann der Level-I 
Klassfikationsfehler als konservativer Schätzer für die Klassifikationsfehler der Level-II und -III Karten betrachtet werden.

Die Ergebnisse der Validierung zeigen, dass die Klassifikationsfehler der Level-I Karten deutlich größer als die entsprechenden Modellfehler sind. Bei einer Betrachtung der Klassifikationsfehler in den Fehlermatrizen (siehe Tabelle 15.3 und 15.3) zeigt sich, dass die Fehler für die einzelnen Klassen sehr unterschiedlich sind. Bei der Interpretation der Fehlermatrizen muss jedoch beachtet werden, dass die Anzahl der Validierungspunkte in den Klassen stark schwankt. Dies ist auf das Stichprobendesign zur Auswahl der Validierungspunkte zurückzuführen. Aufgrund der systematischen Auswahl entspricht die Klassenverteilung der Punkte der Flächenverteilung der Klassen im Untersuchungsgebiet. Im Extremfall führt dies dazu, dass für seltene Klassen (z. B. Urban) kein Validierungspunkt vorhanden ist.

Auch wenn beide Untersuchungsgebiete stark fragmentiert sind, unterscheidet sich die Landnutzung erheblich. In Osa finden sich sehr heterogene Landnutzungsysteme in einem kleinteiligen Mosaik aus Palmöl-, Bananen-, Teak- und Melina-Plantagen, Waldflächen, Weiden und kleinen Ackerflächen. Im Gegensatz dazu wird Guanacaste hauptsächlich durch seine Viehhaltung geprägt, so dass im wesentlichen Wald- und Weideflächen vorkommen. Die unterschiedlichen Landnutzungsformen spiegeln sich auch in der Qualität der Landbedeckungskarten wieder. Die Gesamtgenauigkeit und der Kappawert der Klassifikation sind für Guanacaste deutlich höher (Kappa: 0.75) als in Osa (Kappa: 0.58), wo es verstärkt zu Verwechslungen zwischen den einzelnen Klassen kommt.

Die Aufnahmen in beiden Untersuchungsgebieten werden teilweise durch Wolken und Dunst beeinträchtigt (siehe Abbildung 31 und 32). Dies ist besonders für die Validierung problematisch, da vom Bildinterpreten entschieden werden muss, ob ein bestimmter Punkt der Klasse Cloud zugeordnet wird oder nicht. Dies ist vor allem dann problematisch, wenn es sich um nicht klar abgrenzbare Wolken (z. B. der Gattung Cirrus oder Stratus) handelt. Hier sind teilweise durch die Wolken hindurch Objekte am Boden zu erkennen, deren Signatur jedoch durch die Wolken stark verändert ist.

Durch Anwendung der FAO-Klassendefinitionen auf die Level-I Landkarten (siehe Abbildung 15) wurden die Level-II Karten erstellt. Dabei kommt der Moving-Window-Operator zum Einsatz, der eine fixe Referenzfläche implementiert. Für die vorliegende Arbeit wurde eine Referenzfläche von $3 \times 3 p x$, das entspricht einer Fläche von $9 * 25 \mathrm{~m}^{2}=225 \mathrm{~m}^{2}$, verwendet. 
15.3. Evaluation der Landbedeckungskarten

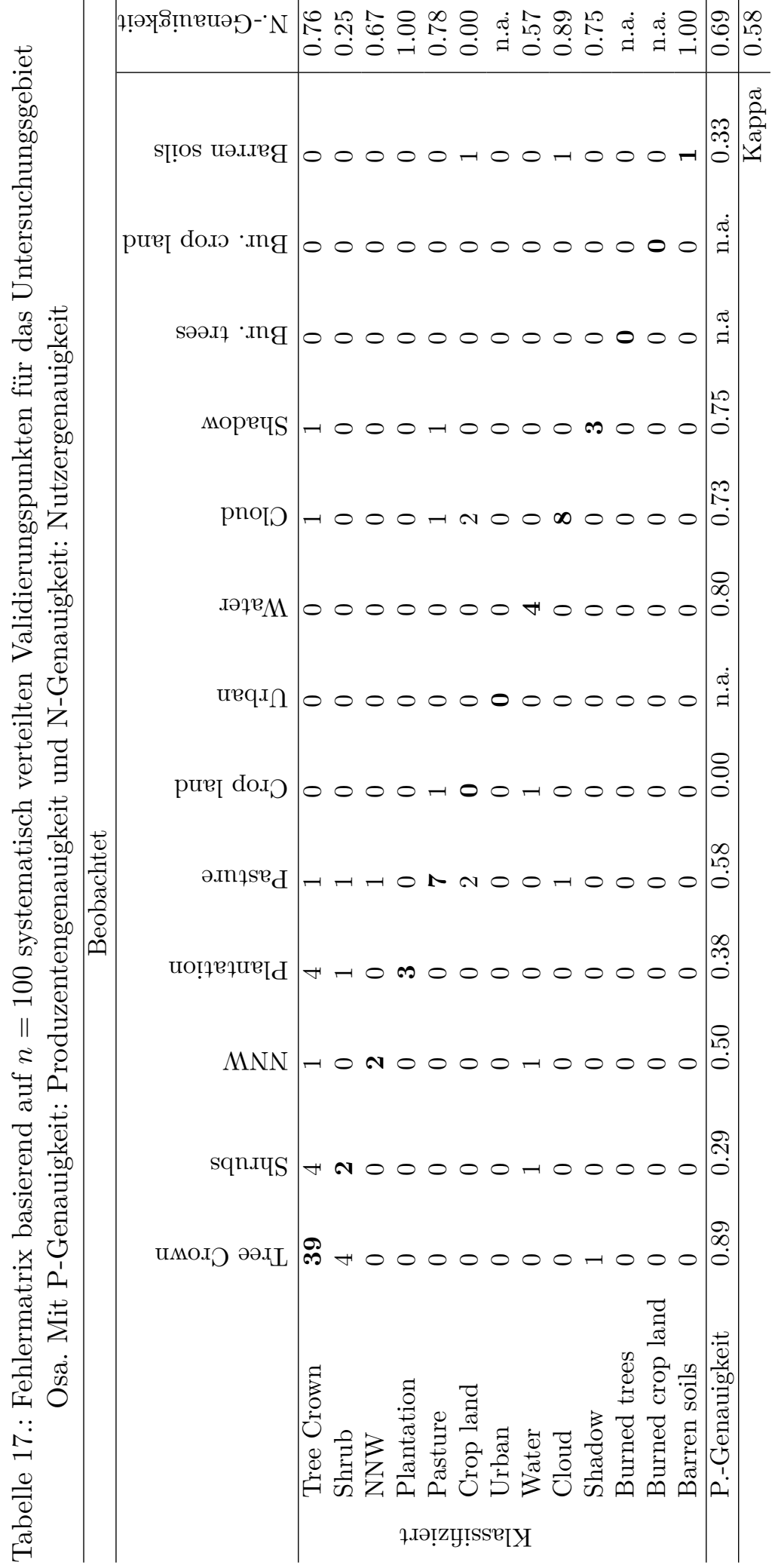




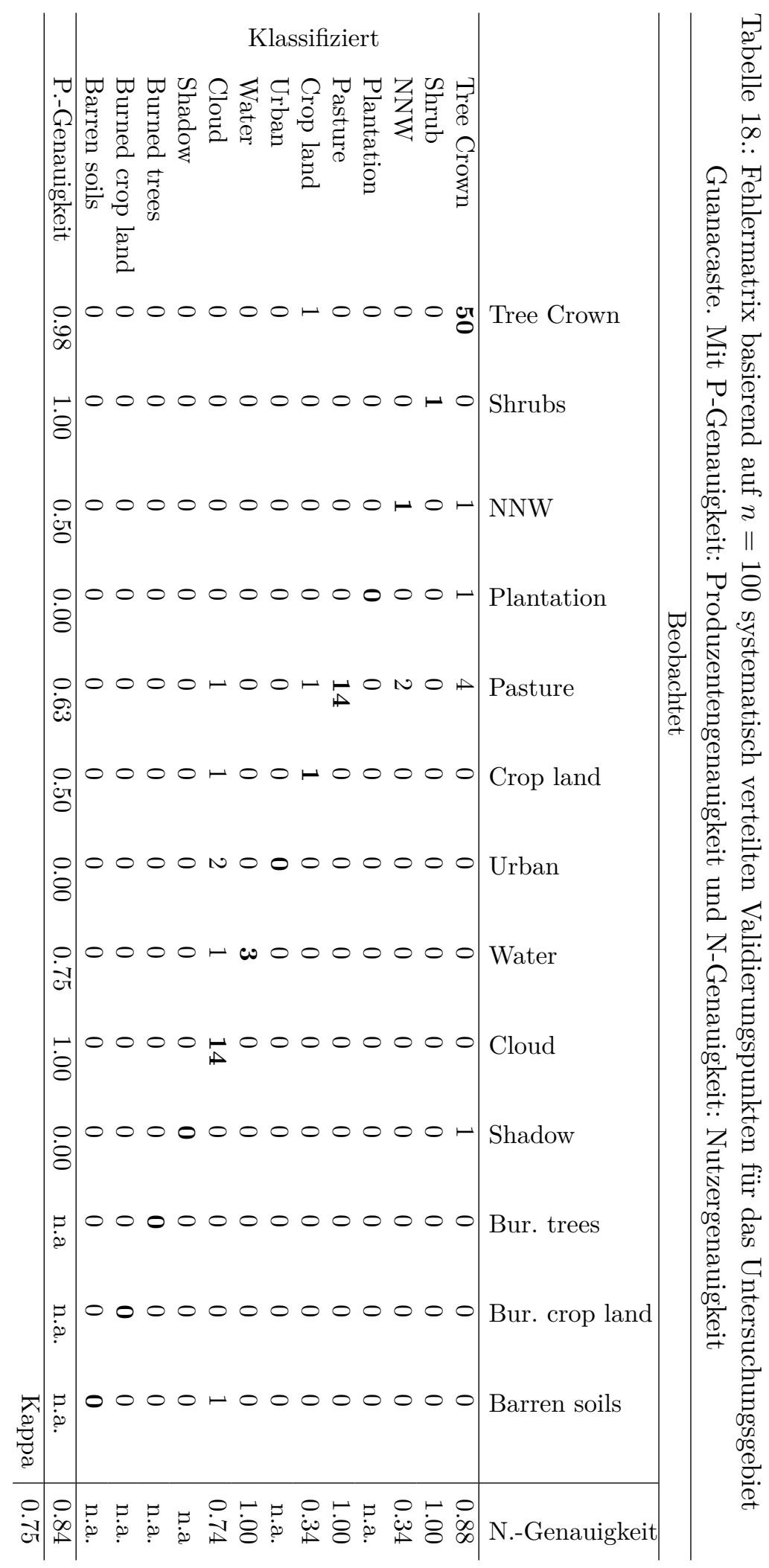




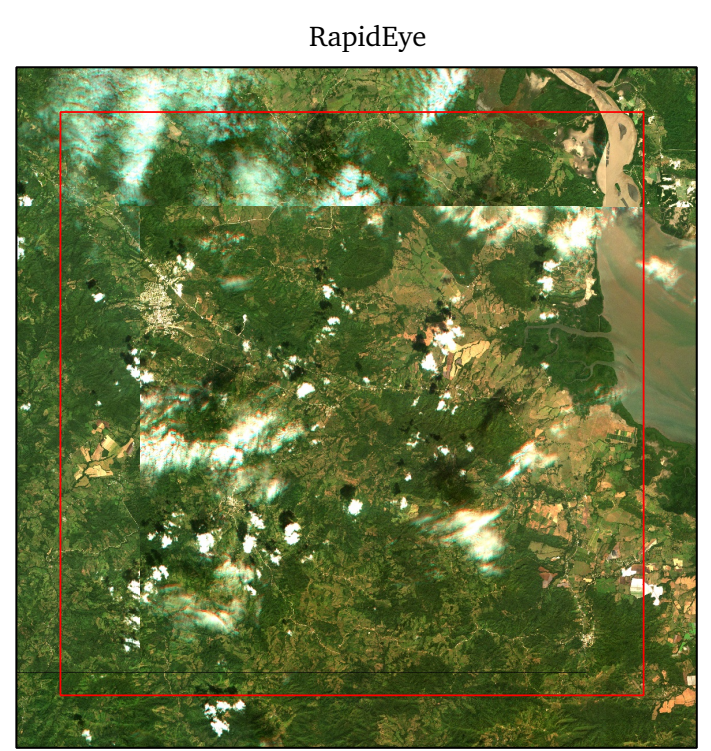

Level II

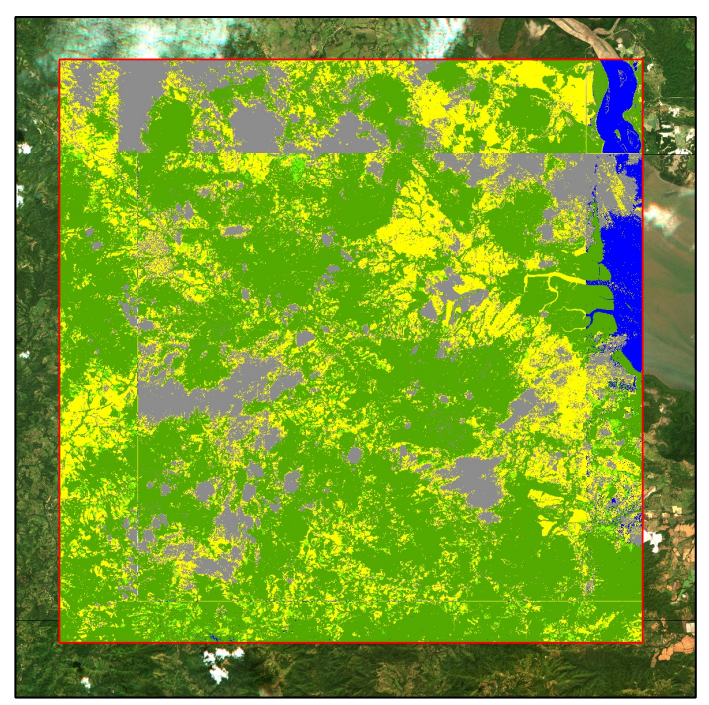

Level I

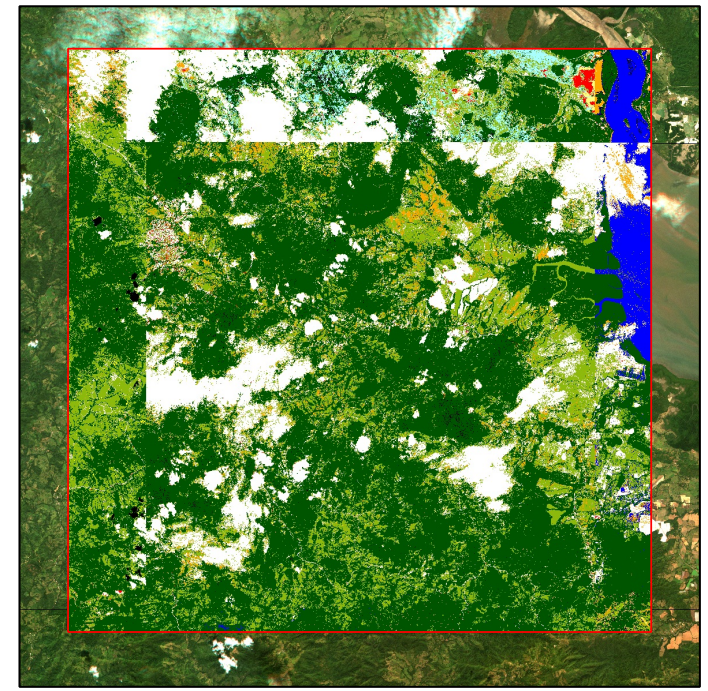

Level III

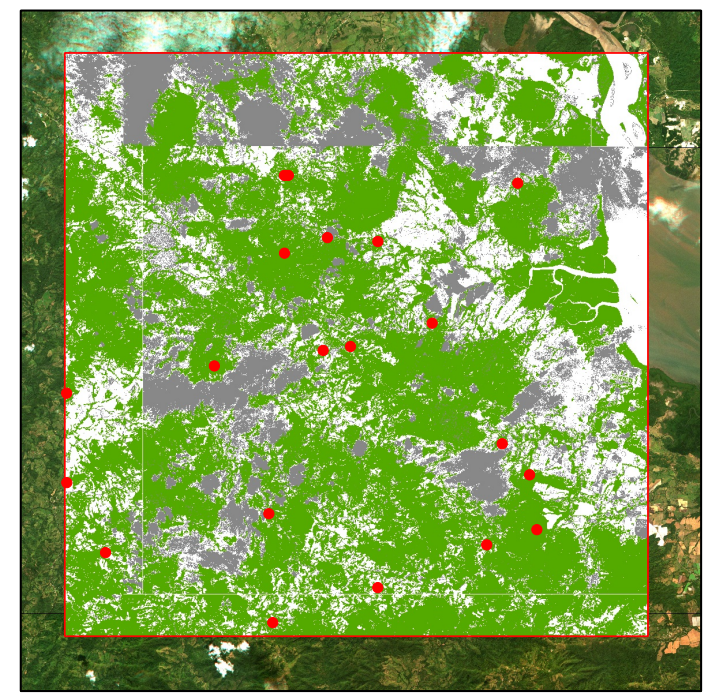

\begin{tabular}{|c|c|c|c|}
\hline Level I & & Level II & Level III \\
\hline Tree Crown & Water & $\square$ No Data & $\square$ Non-Forest \\
\hline Shrub & Cloud & $\square$ Forest & $\square$ Forest \\
\hline$\square$ NNW & Cloud Shadow & Other Land with Tree Cover & $\square$ NoData \\
\hline$\square$ Plantation & Burned tree crowns & Other Land & Fieldplots \\
\hline Pasture & Burned crop land & $\square$ Other Wooded Land & • \\
\hline $\begin{array}{l}\square \text { Cropland } \\
\square \text { Urban }\end{array}$ & $\square$ Baren Ground & Water & \\
\hline
\end{tabular}

Abbildung 31.: Ergebnisse der Klassifizierungen für das Untersuchungsgebiet Guanacaste 


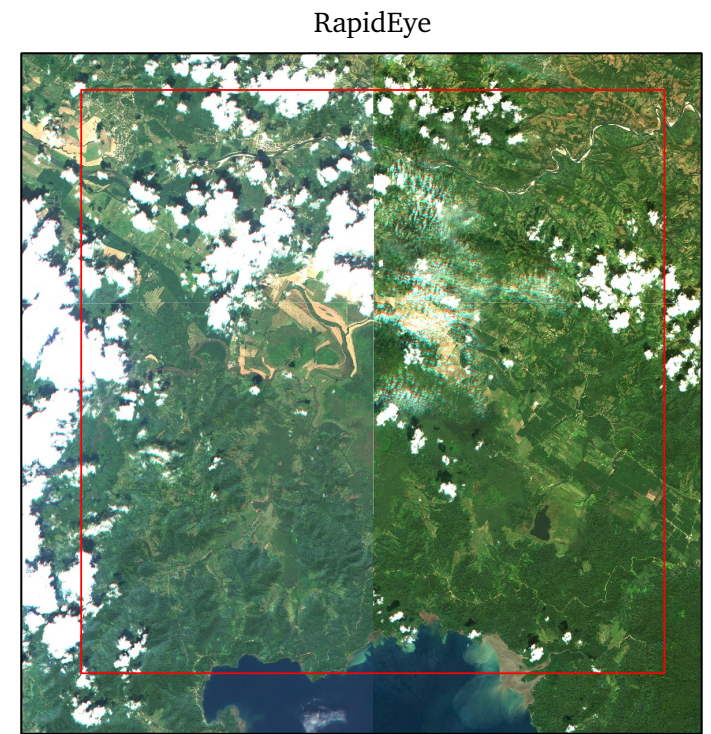

Level II

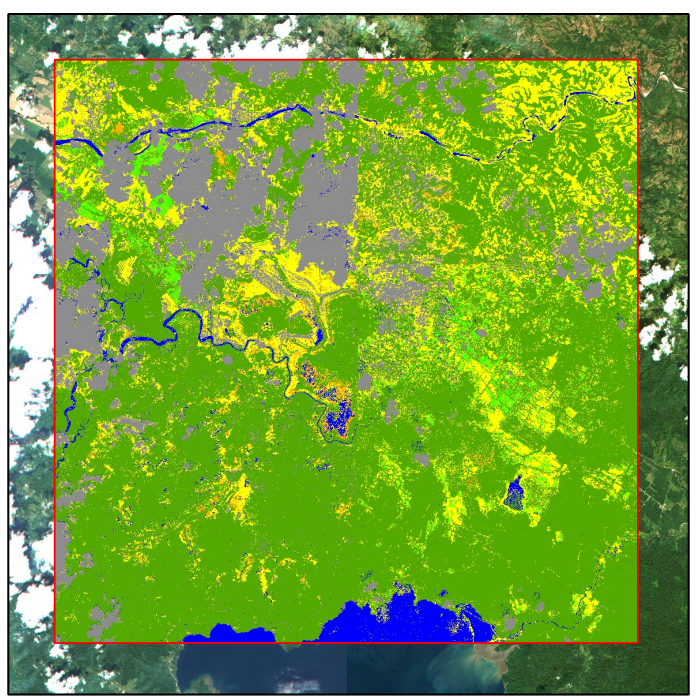

Level I

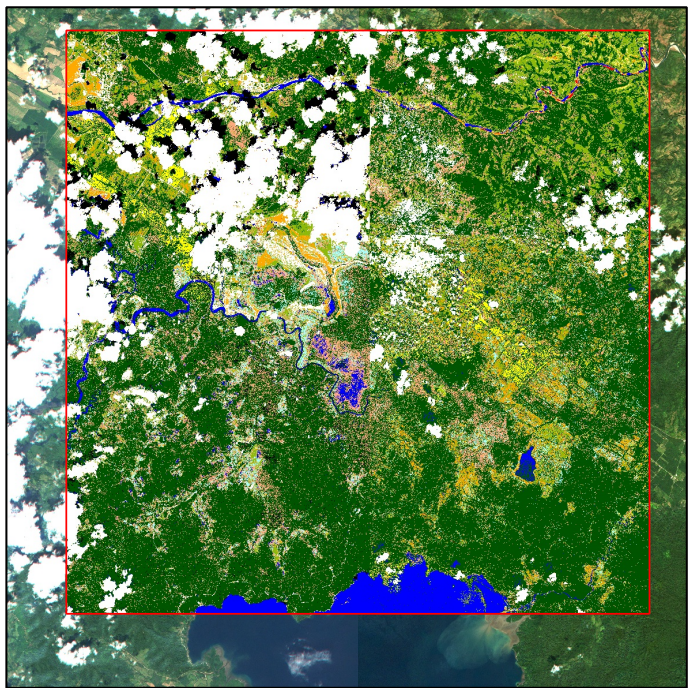

Level III

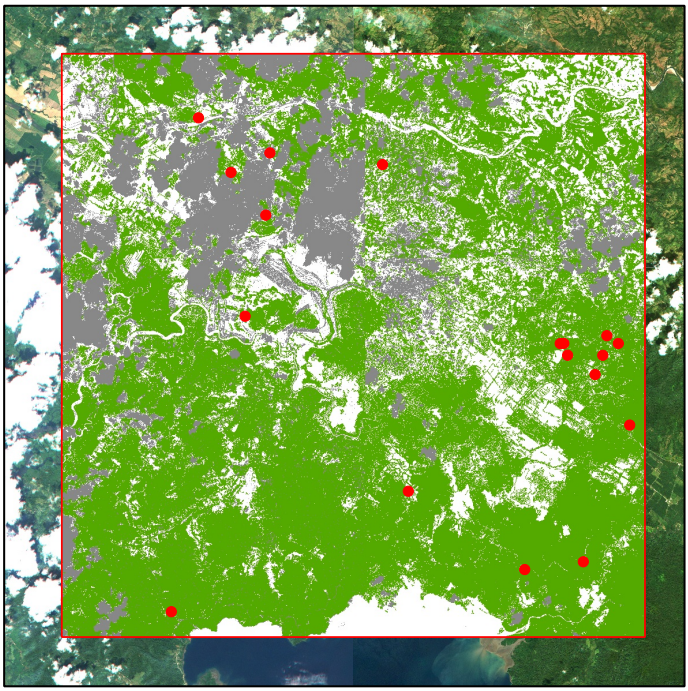

\begin{tabular}{|llll|}
\hline Level I & Level II & Level III \\
$\square$ Tree Crown & $\square$ Water & $\square$ Non-Forest \\
$\square$ Shrub & $\square$ Cloud & $\square$ No Data \\
$\square$ NNW & $\square$ Cloud Shadow & $\square$ Other Land with Tree Cover & $\square$ NoData \\
$\square$ Plantation & Burned tree crowns & $\square$ Other Land & Fieldplots \\
$\square$ Pasture & $\square$ Burned crop land & $\square$ Other Wooded Land & \\
$\square$ Cropland & $\square$ Baren Ground & Water \\
Urban & & \\
\hline
\end{tabular}

Abbildung 32.: Ergebnisse der Klassifizierungen für das Untersuchungsgebiet Osa 
Die Aggregierung der Klassen und die Verwendung einer Referenzfläche führt zunächst zu einer Generalisierung der Karte, so dass einzelne Baumpixel nicht mehr als solche erscheinen, sondern anderen Klassen zugeordnet werden.

Die Waldflächen der Waldkarten (Level-III) entsprechen den Kriterien der FAO-Walddefinition. Neben den Klassen Wald und Nicht-Wald sind auch Flächen vorhanden, die keine Information über die Landbedeckung enthalten. Dies ist vor allem auf den hohen Wolkenanteil in den RapidEye-Bildern zurückzuführen. Zur Berechnung der Waldfläche in Tabelle 19 wurde daher zunächst der Waldanteil für die nicht von Wolken und Wolkenschatten bedeckten Gebiete bestimmt. Die Waldfläche ergibt sich dann aus der Multiplikation des Anteils mit der Größe des Untersuchungsgebietes $\left(900 \mathrm{~km}^{2}\right)$.

Tabelle 19.: Waldflächenschätzung für beide Untersuchungsgebiete abgeleitet aus den LevelIII Karten.

\begin{tabular}{lllll}
\hline Gebiet & Waldanteil & Nicht-Waldanteil & Wolken / Schatten & Waldfläche $\left(\mathrm{km}^{2}\right)$ \\
\hline Osa & $59.7 \%$ & $23.1 \%$ & $17.3 \%$ & 649.404 \\
Guanacaste & $67.1 \%$ & $32.9 \%$ & $17.6 \%$ & 604.048 \\
\hline
\end{tabular}

\subsection{Vergleich der terrestrisch und fernerkundlich gemessenen Kronenüberschirmung}

Die Aufnahmen im Feld fanden im April 2010 statt. Dabei zeigte sich, dass das Anlegen der Transekte besonders in den dicht bewachsenen tropischen Waldgebieten Osas einen erheblichen Aufwand darstellte. Dies war zum einen durch die dichte Vegetation, zum anderen aber auch durch die Topographie bedingt. Die meisten Waldflächen in Osa befinden sich in Gebieten mit steilen Hängen, in denen andere Landnutzungsformen nicht möglich sind. Die Aufnahmen von Transekten an diesen Hängen ist sehr schwierig und teilweise nicht möglich. Abbildung 33 zeigt zwei unterschiedliche Aufnahmesituationen bei den Feldarbeiten. Aufgrund der Schwierigkeiten bei der Aufnahme der Transkete in den Waldgebieten wurde während der Feldaufnahmen entschieden, die Transektlängen für das Stratum drei und vier von $100 \mathrm{~m}$ auf $50 \mathrm{~m}$ zu reduzieren.

Wie in Abschnitt 15.1.2 beschrieben, betrug die Lageabweichung der RapidEye-Bilder bis zu 70m, weshalb sie nach den Feldaufnahmen mit den GCP erneut georeferenziert wurden. 

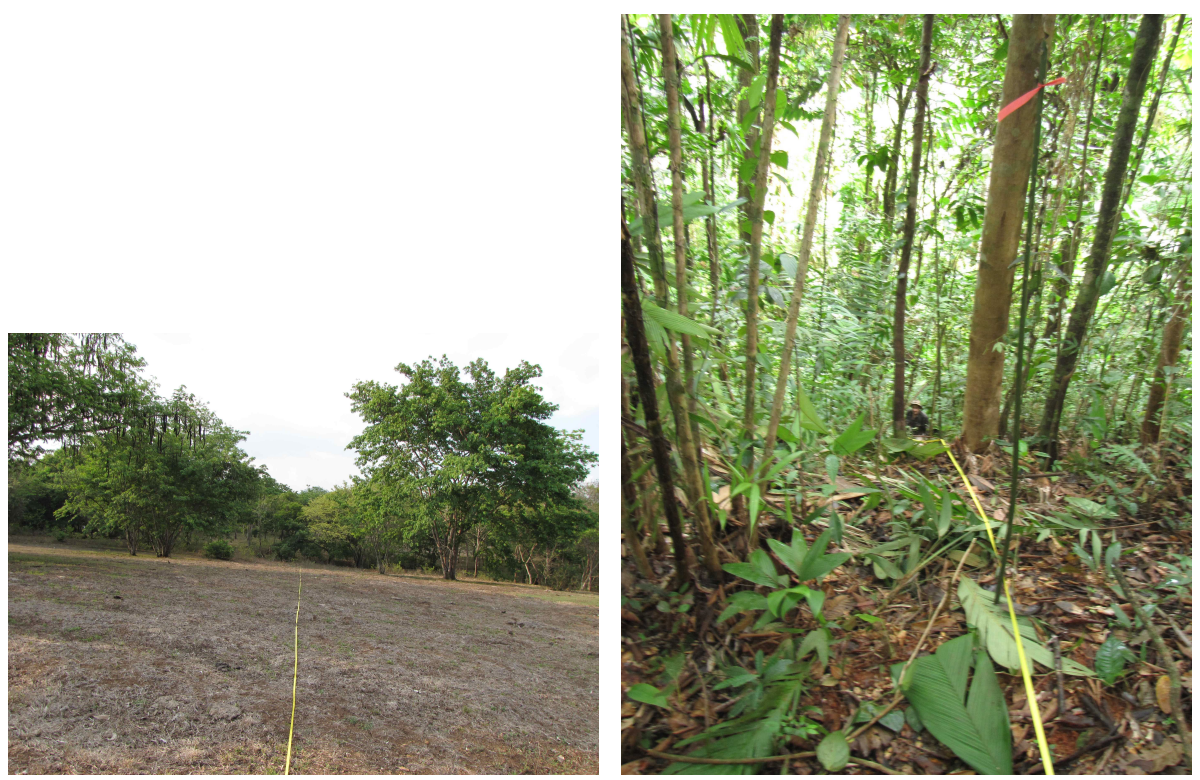

Abbildung 33.: Verschiedene Situationen bei der Aufnahme der Linienstichproben im Feld. Links eine Weide mit Baumbestand in Guanacaste. Rechts: Steilhang in einem dichten Wald in Osa.

Dies erforderte eine erneute Klassifikation der Bilder nach der Georeferenzierung. Dadurch und durch die Verkürzung der Transektlängen kam es zu einer Veränderung der Stratenzuordnung der Probeflächen. Außerdem konnten an einigen Punkten keine Aufnahmen erfolgen, da sie nicht erreichbar waren oder keine Genehmigung des Grundstückseigentümers eingeholt werden konnte. Demnach reduzierte sich die Anzahl der aufgenommenen Stichprobenpunkte. Tabelle 20 gibt die tatsächliche Anzahl und Stratenzuordnung der aufgenommenen Punkte an.

Tabelle 20.: Verteilung der aufgenommenen Probepunkte auf die vier Straten ermittelt aus den Level-I Karten nach der Georeferenzierung

\begin{tabular}{llll}
\hline Stratum & Kronenüberschirmung & Osa & Guanacaste \\
\hline 1 & $<0.25$ & 3 & 0 \\
2 & $\geq 0.25,<0.5$ & 2 & 5 \\
3 & $\geq 0.5,<0.75$ & 4 & 4 \\
4 & $\geq 0.75$ & 9 & 11 \\
\hline & Gesamt & 18 & 20 \\
\hline
\end{tabular}


Für die $n=38$ aufgenommenen Punkte wurde die Kronenüberschirmung aus den Level-I Karten abgeleitet. Um die Kronenüberschirmung aus den Level-I Karten mit den im Feld gemessenen Werten vergleichen zu können, müssen die Referenzflächen identisch sein. Bei den im Feld aufgenommenen Linienstichproben gibt es jedoch keine Referenzfläche im eigentlichen Sinne. Um dennoch einen Vergleich durchführen zu können, wurden als Referenzfläche ein Quadrat mit 200m Kantenlänge für die Straten eins und zwei und 100m Kantenlänge für die Straten drei und vier festgelegt (vergleiche Abbildung 25). Als Schätzer für die terrestrische Kronenüberschirmung diente der Mittelwert des von Baumkronen überschirmten Anteils beider Transekte.

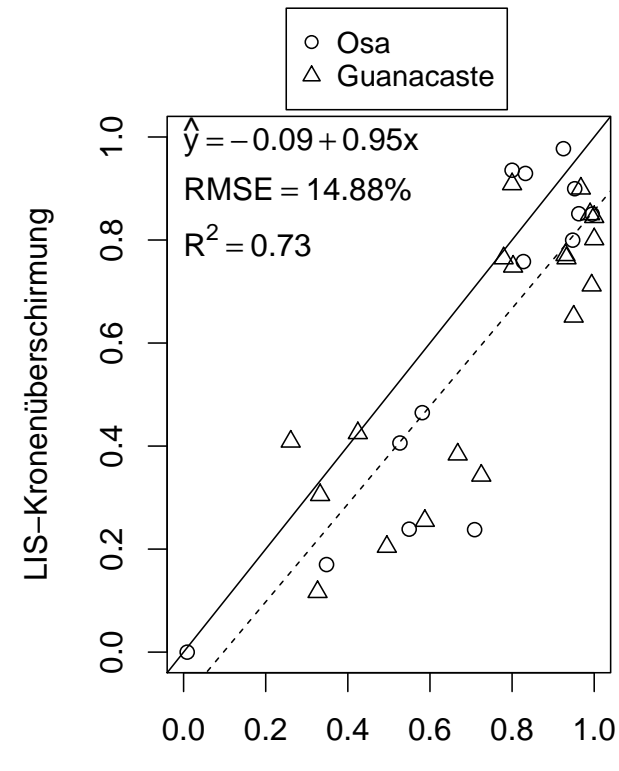

RapidEye-Kronenüberschirmung

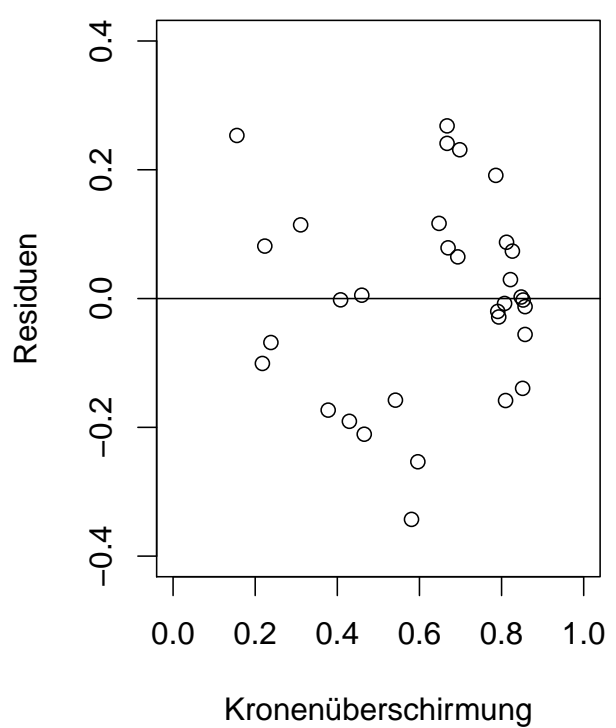

Abbildung 34.: Vergleich der Kronenüberschirmung aus den Linienstichproben (LIS) und den Level-I Karten. Die gestrichelte Linie beschreibt das angepasste lineare Modell. RMSE: Root Mean Square Error.

Wie in Abbildung 34 dargestellt, korrelieren beide Schätzungen der Kronenüberschirmung. Insgesamt kommt es zu einer Überschätzung der Kronenüberschirmung bei der RapidEyeKlassifikation. Trotzdem ist die mittlere Abweichung null, wobei die Residuen homogen über den Wertebereich streuen. Zwischen den beiden Untersuchungsgebieten können keine Unterschiede festgestellt werden, so dass geschlussfolgert werden kann, dass die Klassifikati- 
on in beiden Gebieten ähnlich gut funktioniert. Unter Berücksichtigung der fundamentalen Unterschiede beider Aufnahmemethoden zeigt ein Bestimmtheitsmaß von $R^{2}=0.73$ mit einem $R M S E=14.88 \%$ eine relativ gute Übereinstimmung. 


\section{Diskussion der Fallstudie}

\subsection{Einleitung}

Die Erstellung von Landbedeckungskarten ist ein zentrales Anliegen der fernerkundlichen Erdbeobachtung. Seit dem Start der ersten Erdbeobachtungssatelliten werden die Techniken und Auswertungsmethoden zur Erfassung der Landbedeckung ständig weiterentwickelt. Durch die verstärkte Integration von Fernerkundungstechniken in administrative Aufgaben gewinnt die Standardisierung der Produkte an Bedeutung. Spätestens wenn solche Informationen für die Erfüllung von rechtsverbindlichen Aufgaben und Abkommen genutzt werden, sollte eine Standardisierung und damit verbundene Vergleichbarkeit sichergestellt sein. Erster Schritt zur Standardisierung der Produkte ist die Anwendung einheitlicher Kriterien, Definitionen und Methoden. In der Praxis werden diese häufig in einem politischen Entscheidungsfindungsprozess und nicht durch eine wissenschaftliche Analyse festgelegt. Bartholomé \& Belward (2005) weisen jedoch darauf hin, dass die Standardisierung der Landbedeckungskarten zwar die Konsistenz der Karten erhöht, häufig jedoch zu Akzeptanzproblemen in den einzelnen Ländern führt, da diese Karten nicht der jeweiligen regionalen Walddefinition entsprechen. Dies verdeutlicht, dass verschiedene sich teilweise widersprechende Anforderungen an Landbedeckungskarten gestellt werden. Am Beispiel der Walddefinition lässt sich der Unterschied zwischen der politischen und wissenschaftlich/technischen Vorgehensweise bei der Auswahl der Kriterien gut beobachten. In politischen Verhandlungen wird Wald meistens als eine Form der Landnutzung verstanden. Daher werden auch Flächen, die momentan nicht mit Bäumen bestockt sind als Waldflächen bezeichnet. Aus wissenschaftlicher Sicht stellt eine solche Definition eine Herausforderung dar, da im Rahmen einer monotemporalen Inventur nur schwer festzustellen ist, welche Landnutzung vorliegt.

\subsection{Umsetzung der Kriterien aus der FAO-Walddefinition}

Die Walddefinition der FAO, wie sie im Rahmen des FRA 2010 und der Rio-Abkommen genutzt wird, ist eine Landnutzungsdefinition. Dies drückt sich in der Verwendung von Landnutzungskriterien (z. B. „,orherrschende Landnutzung“, „potentielle Baumhöhe“) aus. Die 
Verwendung von Landnutzungskriterien stellt für die Erfassung der Waldfläche eine Herausforderung dar, da sie sich häufig schwer beobachten lassen. Es gibt verschiedene Gründe die für die Verwendung von Landnutzungskriterien sprechen, auch wenn die Aufnahmen dadurch erschwert werden. Die Landnutzung ist ein wesentlicher Indikator zur Beschreibung des menschlichen Einflusses auf die Umwelt und damit für die Erforschung des globalen Wandels von großer Bedeutung. Daneben gibt es auch praktische Aspekte, die für die Verwendung von Landnutzungskriterien sprechen. So entsprechen Waldflächen, auf denen im Rahmen der normalen Bewirtschaftung Kahlschläge durchgeführt wurden, nach dem Kahlschlag nicht mehr den Landbedeckungskriterien (z. B. Mindestüberschirmung). Aus Sicht des Nutzers stellt der Kahlschlag jedoch keine Änderung der Landnutzung, sondern einen Teil der Waldbewirtschaftung dar. Die FAO-Walddefinition sieht daher vor, temporär unbestockte Flächen als „Wald“ zu klassifizieren, wenn sie innerhalb von fünf Jahren wieder bestockt werden. Um zu entscheiden, ob eine Fläche als Wald zu klassifizieren ist, muss demnach festgestellt werden, ob die Wiederbestockung tatsächlich innerhalb der Frist stattfindet und ob die ausgewachsenen Bäume eine Höhe von $5 \mathrm{~m}$ erreichen. Beide Kriterien können anhand einer einzigen Beobachtung vor Ort nicht bestimmt werden. Neben der Walddefinition spielt die Landnutzung auch bei der Definition der Walddegradierung im Rahmen des REDD Programms eine wichtige Rolle, wie sich gut an der kontroversen Diskussion über die geeigneten Kriterien und Schwellenwerte nachvollziehen lässt (Guariguata et al., 2009; Karjalainen et al., 2003; Putz \& Redford, 2010; Putz \& Sasaki, 2009; Schoene et al., 2007). Berücksichtigt man ausschließlich den Rückgang der Kronenüberschirmung in einem bestimmten Zeitraum, wie es vom IPCC (Karjalainen et al., 2003) vorgeschlagen wurde, müssten viele nachhaltig bewirtschaftete mitteleuropäische Wälder als degradiert angesehen werden. Daher schlagen Sasaki \& Putz (2009) vor, zumindest auf nationaler Ebene auch sozioökonomische Waldfunktionen, sprich Landnutzung, als Kriterium für die Degradierung von Wäldern zu berücksichtigen.

Die fernerkundliche Erfassung der Landnutzung ist relativ neu und bisher wenig fortgeschritten. Im dritten Teil der vorliegenden Arbeit wurde ein Verfahren entwickelt, das es ermöglicht Landnutzungskarten zu erstellen, die auf den Kriterien der FAO-Walddefinition beruhen. Als methodischer Ansatz wurde ein hierarchischer Klassifikationsschlüssel gewählt, welcher zunächst verschiedene Landbedeckungsklassen (Level-I) wie Baumgruppen, Sträucher und Wasserflächen identifiziert. Anschließend wurden diese Landbedeckungsklassen durch ein Regelwerk, das in Form eines Entscheidungsbaums implementiert ist, in Beziehung zueinander gesetzt, um sie in Landnutzungsklassen zu überführen. Ein ähnlicher hier- 
archischer Ansatz wurde auch von Martínez \& Mollicone (2012) verfolgt. Anstelle von Entscheidungsbäumen verwenden die Autoren „hierarchische Ketten“ mit festgelegten Schwellenwerten. Leider geht aus ihrer Arbeit nicht hervor wie diese festgelegt werden können. Ein Nachteil der Methode von Martínez \& Mollicone (2012) ist, dass die Referenzfläche nicht unabhängig von der Mindestfläche definiert werden kann. Beide sind jedoch verschiedene Kriterien der Walddefinition. Demgegenüber bietet das in der vorliegenden Arbeit entwickelte Referenzflächenverfahren folgende Vorteile:

- Die Referenzfläche und Mindestgröße können getrennt festgelegt werden.

- Auf der Referenzfläche können die Kriterien Mindestüberschirmung und vorherrschender Landnutzungstyp bestimmt werden.

- Die Verwendung eines Moving-Window-Operators ermöglicht eine Schätzung für jedes Pixel und liefert somit kontinuierliche Schätzungen der Kronenüberschirmung.

- Das Verfahren ist kompatibel mit terrestrischen Aufnahmen, da die selben Referenzflächen verwendet werden können.

Die Kriterien und Schwellenwerte der Walddefinitionen werden derzeit weiterhin verhandelt, so dass davon auszugehen ist, dass sie sich kontinuierlich ändern. Durch die Trennung von Bildklassifikation und Anwendung der Kriterien bietet das entwickelte Verfahren die Möglichkeit, die Schwellenwerte nach der einmaligen Bildklassifikation einfach zu ändern. Außerdem lassen sich so weitere Kriterien ohne großen Aufwand hinzuzufügen. So könnte als weiteres Kriterium die Mindestbreite, welche in der vorliegenden Arbeit nicht berücksichtigt wurde, in das Regelwerk integriert werden. Weitere Vorteile der Trennung von Klassifikation und Anwendung der Kriterien ist, dass das Verfahren nicht auf einen bestimmten Sensor festgelegt ist. Die Landbedeckungskarten (Level-I) können mit verschiedenen hochaufgelösten multispektralen Sensoren erstellt werden. Auch der Klassifikator kann beliebig gewählt werden, solange er die Differenzierung der Landbedeckungsklassen gestattet. Durch diese Flexibilität lässt sich das Verfahren gut an sehr unterschiedliche Umweltbedingungen anpassen, wie der Vergleich der Ergebnisse zwischen Costa Rica und Burkina Faso zeigt (Magdon et al., 2013). 


\subsection{Erstellung der Waldkarten mit dem ForestEye-Prozessor}

\subsubsection{Eignung der RapidEye-Bilder}

Im vierten Teil der Arbeit wurde das entwickelte Verfahren mit Daten des RapidEyeSatellitensystems auf seine praktische Anwendbarkeit in zwei Untersuchungsgebieten in Costa Rica getestet. Dabei zeigte sich, dass die Bilder aufgrund ihrer hohen spektralen, räumlichen und temporären Auflösung sowie der globalen Abdeckung für Waldmonitorings im Rahmen der internationalen Umweltabkommen zweckmäßig sind. Die nominale Auflösung von $6.5 \mathrm{~m}$, die während der Orthorektifizierung auf $5 \mathrm{~m}$ reduziert wurde, ist geeignet große einzelne Bäume, Baumgruppen und Baumreihen zu erkennen und somit die Kronenüberschirmung zu erfassen. Der Vergleich mit den terrestrischen Messungen der Kronenüberschirmung mit einem Bestimmtheitsmaß von $R^{2}=0.74$ bestätigt dies. Hansen et al. (2003) klassifizierten Baumkronen in IKONOS-Daten (räumliche Auflösung: 4m) mit einer Modellgenauigkeit von 86.7\%. Die so klassifizierten Daten wurden dann auf 30m Landsatpixel aggregiert. Der Vergleich mit der im Feld gemessenen Kronenüberschirmung zeigte einen, zur vorliegenden Studie vergleichbaren, Wert des Bestimmtheitsmaßes von $R^{2}=0.74$. Goetz et al. (2003) ermittelten Klassifikationsgenauigkeiten von $97.3 \%$ für die Unterscheidung der Klassen „Bäume“, „Nicht-Bäume“ und „Wolken“ in multispektralen IKONOS-Bildern. Diese Studien zeigen, dass die räumliche Auflösung im Bereich von $5 \mathrm{~m}$ grundsätzlich ausreichend ist, um die Kronenüberschirmung in den untersuchten Waldtypen zu ermitteln. Gleichzeitig bleiben die Datenmengen relativ gering, so dass auch größere Flächen bearbeitet werden können. Für multispektrale RapidEye-Bilder mit 16bit ergibt sich zum Beispiel eine Datenmenge von ca. $0.4 \mathrm{MB} / \mathrm{km}^{2}$.

\subsubsection{Bildkalibrierung}

Bestandteil des entwickelten Verfahrens ist die Bildkalibrierung und Bildverbesserung. Prinzipiell können zwei Ansätze zur atmosphärischen Korrektur unterschieden werden, die absolute und die relative Korrektur. In der vorliegenden Arbeit wurde eine absolute Korrektur mit dem 6S-Strahlungstransfermodell durchgeführt. Diese hat den Vorteil, dass die Messungen am Sensor in physikalisch interpretierbare Größen überführt werden, was eine einfache Interpretation der Werte ermöglicht und einen Vergleich mit Daten anderer Sensoren erlaubt. Für die absolute Korrektur werden jedoch Informationen über die 
Atmosphärenzusammensetzung benötigt. Diese können entweder aus dem Bild selbst oder aus externen Quellen gewonnen werden. In der vorliegenden Arbeit wurden die Informationen über den Zustand der Atmosphäre aus den MODIS-Produkten gewonnen. Das ist ein neuer Ansatz, der maßgeblich von Dr. H. Fuchs aus der Abteilung Waldinventur und Fernerkundung, Georg-August-Universität Göttingen, entwickelt wurde. Die Verwendung von MODIS-Produkten hat mehrere Vorteile i) die MODIS-Produkte haben eine globale Abdeckung mit einer hohen zeitlichen Auflösung (täglich), ii) die Daten liegen in einem einheitlichen Standard vor und iii) die MODIS-Produkte werden von der NASA frei zur Verfügung gestellt. Neben diesen Vorteilen ergeben sich auch Nachteile, wenn die Atmosphäreninformationen aus Satellitendaten gewonnen werden. Zum einen treten die typischen Probleme mit Wolken und Wolkenschatten auf, was dazu führen kann, dass für das jeweilige Gebiet keine oder nur vereinzelt Informationen zur Verfügung stehen, wie es in Guanacaste der Fall ist (vergl. Tabelle 14). Zum anderen können zeitliche Differenzen zwischen beiden Aufnahmen auftreten. Für die Testgebiete in Costa Rica war die zeitliche Differenz gering, aber für andere Gebiete ist das nicht sichergestellt, da die RapidEye- und MODIS-Satelliten nicht synchronisiert sind. Aufgrund des hohen Aufwandes, der mangelhaften Korrektur und der teilweise unplausiblen Reflexionswerte muss geschlussfolgert werden, dass die Korrektur der RapidEye-Daten mit dem durch MODIS-Daten parametrisierten 6-Modell noch nicht zufriedenstellend funktioniert. Zur Entwicklung eines operationalen Verfahrens sollten daher vor allem die Verfahren, welche Informationen aus dem Bild für die Korrektur nutzen, weiter untersucht werden. El Hajj et al. (2008) verglichen eine relative Korrektur auf Basis von linearen Modellen mit der absoluten Korrektur durch das 6S-Modell und zeigen, dass die relative Korrektur ähnlich gute Ergebnisse liefert. Hadjimitsis et al. (2004) verglichen neun verschiedene Verfahren und schlussfolgern, dass die wesentlich leichter zu implementierenden Dark-Object-Pixel (DOP)-Ansätze zu besseren Ergebnissen führen. Diese Korrektur basiert auf dem Vergleich von invarianten Objekten im Bild. Da sich die RapidEye-Bilder nur um 500m überdecken ist nicht sichergestellt, dass solche Objekte in den Überlappungsbereichen vorkommen. In diesem Fall könnten auch Histogrammanpassungen über den gesamten Überdeckungsbereich erfolgen, wenn zuvor die Wolken und Wolkenschatten ausmaskiert wurden.

Die Detektion der Wolken und Wolkenschatten erfolgte in der vorliegenden Arbeit während der Level-I Klassifikation mit einer hohen Genauigkeit, wenn in den Trainingsdaten ausreichend Pixel beider Klassen vorhanden waren. Insofern kann geschlussfolgert werden, dass die überwachte Klassifikation der RapidEye-Bilder für die Erstellung von Wolkenmas- 
ken geeignet ist. Magdon et al. (2013) zeigten, dass die Detektion der Wolken im Falle von dünnen Schleierwolken jedoch problematisch sein kann. In Fällen, in denen die Wolkendecke dünn ist und die Landbedeckung darunter noch erkannt werden konnte, kam es zu Unterschieden bei der visuellen Interpretation und der automatischen Klassifikation. Dabei liegt das Problem in der Definition von Wolken. Die räumliche Konzentration von Wassertropfen folgt einem Kontinuum und eine Abgrenzung lässt sich nur schwer rechtfertigen.

\subsubsection{Random Forests Klassifikator}

Die Klassifikation der RapidEye-Bilder erfolgte mit dem RF-Klassifikator und zeigte sehr geringe Modellfehler, die vergleichbar mit anderen Studien sind, in denen Landbedeckungskarten aus multispektralen Satellitenbildern mit dem RF-Klassifikator erstellt wurden (Gislason et al., 2006; Pal, 2005; Rodriguez-Galiano et al., 2012). Zwei Parameter mussten für die Klassifikation der RF-Parameter festgelegt werden: i) die Anzahl an Klassifikationsbäumen $n$ und ii) die Anzahl der potentiellen Prädiktoren $m$. Zur Festlegung des ersten Parameters wurden RF-Modelle mit unterschiedlicher Anzahl an Entscheidungsbäumen erzeugt. Die Ergebnisse zeigen den von Breiman (2001) beschriebenen Verlauf, wobei der OOBFehler mit zunehmender Anzahl der Entscheidungsbäume konvergiert. In der vorliegenden Studie kann die Konvergenz ab ca. 100 Bäumen beobachtet werden (siehe Abbildung 29), was übereinstimmt mit den Ergebnissen von Rodriguez-Galiano et al. (2012). Auch Pal (2005) zeigte, dass sich der OOB-Fehler nur noch um weniger als 1\% reduzierte, wenn statt 100, 12000 Entscheidungsbäume verwendet werden. Der zweite Parameter beeinflusst zum einen die Stärke des einzelnen Entscheidungsbaums, zum anderen die Unabhängigkeit der Entscheidungsbäume untereinander. Ein hoher $m$-Wert führt zu einem stärkeren Entscheidungsbaum und zu einer höheren Abhängigkeit der Bäume. Daher schlägt Breiman (2001) vor, für jedes RF-Modell den geeignetsten Wert für $m$ individuell anzupassen. In der vorliegenden Arbeit wurde der Wert für alle RF-Modelle pauschal auf $m=\sqrt{M}$ festgelegt. Rodriguez-Galiano et al. (2012) zeigten, dass $m$ nur dann einen signifikanten Einfluss hat, wenn die Anzahl der Entscheidungsbäume so gering ist, dass noch keine Konvergenz erreicht wurde $(n<100)$. Insofern kann davon ausgegangen werden, dass durch die starre Festlegung des $m$ Wertes keine Verringerung der Genauigkeit der Klassifikationsmodelle eingetreten ist, auch wenn dies nicht explizit untersucht wurde.

Ein wesentlicher Vorteil des RF-Klassifikators ist die Möglichkeit, auf Grundlage des bagging-Verfahrens die Bedeutung der einzelnen Prädiktoren zu bestimmen. Die Variablen- 
selektion ist speziell für die Auswertung multispektraler Bilder ein wichtiger Aspekt in der Fernerkundung. Häufig stehen eine begrenzte Anzahl von Trainingsdaten einer sehr großen Anzahl von möglichen Prädiktoren gegenüber. Da die Prädiktoren aus den Bildern abgeleitet werden, korrelieren sie stark und tragen somit redundante Informationen. Der RFKlassifikator stellt eine Möglichkeit zur Beurteilung der Prädiktorbedeutung zur Verfügung, welche sich einfach in den Klassifikationsprozess integrieren lässt. Einige Aspekte müssen jedoch kritisch betrachtet werden:

- Gislason et al. (2006) hinterfragen die zufällige Auswahl von $m$ Prädiktoren, da nicht sichergestellt ist, dass jeder Prädiktor mindestens einmal gewählt wird. Dies kann dazu führen, dass ein geeigneter Prädiktor zufällig nie ausgewählt wird und somit als unbedeutend eingestuft wird. Dies ist besonders kritisch, wenn sehr viele Prädiktoren und nur wenige Entscheidungsbäume verwendet werden.

- Verschiedene Studien weisen darauf hin, dass die VI Statistiken verzerrt sein können. Strobl et al. (2007) zeigten, dass die GVI verzerrt ist, wenn sich die Prädiktoren in ihrem Wertebereich oder der Anzahl der Ausprägungen stark unterscheiden. Dabei wird die Bedeutung von Prädiktoren mit einer größeren Anzahl an Ausprägungen systematisch überschätzt. Dies gilt nicht für die PVI, da sie nicht aus der Auswahlhäufigkeit der Kriterien, sondern über die Änderung der Klassifikationsfehler vor und nach der Permutation bestimmt wird. Boulesteix et al. (2012) weisen jedoch daraufhin, dass Prädiktoren mit vielen Ausprägungen zu einer höheren Varianz der PVI führen. Die in der Arbeit verwendeten spektralen Bänder, Vegetations- und Texturindices sind alle kontinuierlich, unterscheiden sich jedoch in ihrem Wertebereich. Zum Beispiel ist der Wertebereich der spektralen Reflexion $0 \leq x \leq 1$, während der NDVI durch die Normierung einen Wertebereich von $-1 \leq x \leq 1$ hat. Für die Texturen ergeben sich sehr unterschiedliche Wertebereiche, die im wesentlichen durch die Anzahl der Grauwertstufen bei der Berechnung der GLCM beeinflusst wird. Vor diesem Hintergrund erscheint es sinnvoll bei der Weiterentwicklung des Verfahrens eine Normierung aller Prädiktoren auf einen einheitlichen Wertebereich durchzuführen.

\subsubsection{Qualität der Karten}

Die Ergebnisse zeigen große Unterschiede zwischen den Modell- und Kartengenauigkeiten, wobei letztere wesentlich heterogener und niedriger sind. Im Kontext der Waldflächenerfas- 
sung ist das unbefriedigend, da nicht die Modelle sondern die Karten von Interesse sind. Daher stellt sich die Frage nach den Ursachen für die große Differenz, besonders wenn in anderen vergleichbaren Studien (Rodriguez-Galiano et al., 2012) wesentlich geringere Unterschiede beobachtet wurden. Verschiedene Interpretationen sind möglich:

- Die Differenzen können auf die Verteilung und den Wertebereich der Pixelwerte in den Trainings- und Testdatensätzen (Bilder) zurückgeführt werden. Wenn der Wertebereich des Test- größer als der des Trainingsdatensatzes ist, kommt es zu einer Extrapolation durch den RF-Klassifikator. Da dieser jedoch auf festen Schwellenwerten beruht, die in der Trainingsphase aus den Trainingsdaten abgeleitet werden, ist die Genauigkeit der Extrapolation unbekannt. Daher sollte eine Extrapolation grundsätzlich vermieden werden.

- Die Differenzen können auf die Verteilung der Landbedeckungsklassen im Trainingsdatensatz zurückgeführt werden. Viele überwachte Klassifikationsverfahren reagieren sensitiv auf ungleich verteilte Trainingsdaten (Dupret \& Koda, 2001; Weiss \& Provost, 2001). Der RF-Klassifikator maximiert die Gesamtgenauigkeit des Modells. Da die falsche Klassifikation einer häufig vorkommenden Klasse den Modellfehler stärker erhöht als die fehlerhafte Klassifikation einer selten vorkommenden Klasse wird das RF-Modell so trainiert, dass es die häufige Klasse besser klassifiziert. Daher kann davon ausgegangen werden, dass die Modellgenauigkeit verzerrt ist.

- Die Differenzen können auf die Verteilung der Landbedeckungsklassen im Validierungsdatensatz zurückgeführt werden. Wenn die Klassenverteilung des Validierungsdatensatzes ungleich ist, wird die Aussagekraft der Fehlermatrix eingeschränkt. Wenn z. B. nur ein Validierungspunkt vorhanden ist, kann die Klassifikationsgenauigkeit nur 0\% (bei einer fehlerhaften Klassifikation) oder 100\% (bei einer korrekten Klassifikation) betragen.

Alle drei genannten Gründe sind auf die Auswahl der Referenzdaten zurückzuführen. Diese erfolgte sowohl für die Trainings- als auch für die Validierungsdaten mit einem systematischen Stichprobendesign. Dies hat die Eigenschaft, dass die Verteilung der Klassen in der Stichprobe den räumlichen Anteilen der Klassen im Untersuchungsgebiet entspricht (Selbststratifizierung). Im konkreten Fall führte dies dazu, dass in Osa und Guanacaste die meisten Referenzdaten der Klasse „Baumkrone“ angehörten, da in beiden Gebieten diese Klasse den größten Flächenanteil an der Landschaft hat. Landbedeckungsklassen wie 
„Wasserflächen“ und „Siedlungsflächen“ waren nur gering vertreten, was zu niedrigen Klassifikationsgenauigkeiten dieser Klassen führte (vergl. Tabelle 15.3 und 15.3). Da zu erwarten ist, dass eine gleichmäßigere Verteilung der Landbedeckungsklassen in den Referenzdaten die Kartengenauigkeit erhöht, sollten im Zuge der Weiterentwicklung des Verfahrens Optionen untersucht werden, die zur Verbesserung der Verteilung beitragen. Auf folgenden drei Ebenen könnte angesetzt werden:

\section{Stichprobenverfahren für die Auswahl der Referenzdaten}

Die erste Möglichkeit zur Verbesserung der Verteilung des Referenzdatensatzes ist eine Änderung des Auswahlverfahrens. Die Verwendung des systematischen Stichprobenverfahrens hat den Nachteil, dass die Verteilung der Klassen ihren Flächenanteilen im Untersuchungsgebiet entspricht. Somit führt eine einfache systematische Stichprobe zwangsläufig zu einer Ungleichverteilung der Referenzdaten, wenn nicht alle Klassen gleichmäßig im Untersuchungsgebiet vertreten sind. Bei der Erstellung von Landbedeckungskarten ist das selten der Fall, da bestimmte Klassen häufig nur auf geringer Fläche vorkommen (z. B. Siedlungsflächen, Wasserflächen, Wolkenschatten). An dieser Stelle sollte erwähnt werden, dass der Einsatz von Stichprobenverfahren einen Fortschritt gegenüber der subjektiven Auswahl darstellt, wie sie in vielen fernerkundlichen Studien üblich ist. Im Gegensatz zur subjektiven Auswahl „repräsentativer" Gebiete kann durch die Anwendung eines wahrscheinlichkeitsbasierten Auswahlverfahrens erwartet werden, dass die Referenzdaten die Population des Untersuchungsgebietes widerspiegeln. Dies ist z. B. von Bedeutung, wenn anhand der Fehlermatrix auf die Güte der Klassifikation im Untersuchungsgebiet geschlossen werden soll. Hierbei wird angenommen, dass die beobachteten Klassifikationsergebnisse in der Fehlermatrix (Stichprobe) den Ergebnissen der Bildklassifikation entsprechen (Population)(Stehman, 2000).

Verschiedene Studien untersuchen den Einfluss des Stichprobendesigns auf die Schätzung der Kartengenauigkeit. Congalton (1988) verglich fünf verschiedene Designs zur Erstellung von Validierungsdaten anhand von simulierten Satellitenbildern für drei Landschaftstypen. Er zeigte, dass die Wahl des geeignetsten Stichprobendesigns vom Landschaftstyp abhängt. Auch Chen \& Wei (2009) untersuchten in einer Simulationsstudie verschiedene Stichprobendesigns. Sie konnten zeigen, dass die Klassenverteilung einen größeren Effekt als die Landschaftsstruktur (Autokorrelation) auf die Wahl des besten Stichprobenverfahrens hat. Ihre Simulationen bestätigen auch das Ergebnis der vorliegenden Arbeit, nachdem systematische Stichproben wenig geeig- 
net sind die Klassifikationsgenauigkeit der einzelnen Klassen zu beschreiben, wenn diese ungleich verteilt sind. In solchen Fällen ist die Verwendung von stratifizierten Stichprobendesigns wesentlich effektiver, da so eine Gleichverteilung der Klassen in den Referenzdaten erreicht werden kann. Stehman (2004) untersuchte verschiedene Optionen zur Kombination von Stratifizierten- und Klumpenstichproben für die Validierung der nationalen Landbedeckungskarten in den USA. Er konnte zeigen, dass durch die Kombination beider Verfahren auch für seltene Klassen Nutzergenauigkeiten mit hoher Präzision geschätzt werden können.

Im Gegensatz zu den Validierungsdaten ist der Einfluss verschiedener Stichprobenverfahren zur Erstellung von Trainingsdaten auf die Klassifikationsgenauigkeit bisher wenig untersucht worden. Ein Grund dafür ist, dass der Einfluss des Stichprobendesigns wesentlich vom verwendeten Klassifikator abhängt. Werden parametrische Klassifikationsverfahren, wie zum Beispiel der Maximum-Likelihood (ML)-Klassifikator verwendet, muss der Trainingsdatensatz geeignet sein die Mittelwerte und Varianz-Kovarianz Matrix für jede Klasse zu schätzen (Tso \& Mather, 2009). Bei nicht parametrischen Verfahren kann es wichtig sein Informationen aus den jeweiligen Klassengrenzen zu selektieren, wie Foody \& Mathur (2006) für Support Vector Maschines (SVM) zeigten. Hier könnte vor der Auswahl der Trainingsdaten eine unüberwachte Klassifizierung vorgeschaltet werden, um auf diese Weise spektrale Straten zu definieren, aus welchen dann die Trainingsdaten gezogen werden. Bei diesem Ansatz stellt sich jedoch die Frage wie viele Klassen/Straten in der unüberwachten Klassifikation unterschieden werden müssen, um einen geeigneten Trainingsdatensatz zu erhalten.

\section{Anpassung der Referenzdatenverteilung}

Durch eine nachträgliche Stratifizierung (engl. post stratification) der Referenzdaten mit anschließender Zufallsauswahl könnte ein neuer Datensatz mit Gleichverteilung der Klassen erstellt werden. Dabei würden aus den häufigen Klassen nur einige Stichproben gezogen (down sampling) und die Stichproben in den seltenen Klassen teilweise mehrfach ausgewählt (over sampling). Solche Verfahren wurden unter anderen von Chen et al. (2004); De Fries et al. (1998) und Liu et al. (2006) vorgeschlagen. Letztere testeten vier Stichprobendesigns zur Anpassung der Klassenverteilung und kamen zu dem Ergebnis, dass die Modellgenauigkeit in allen Fällen erhöht wird. Diese Verfahren können die Klassenverteilung anpassen. Für den Fall, dass eine seltene Landbedeckungsklasse nicht im Referenzdatensatz ist, können sie jedoch nicht verwendet werden. 


\section{Anpassung des RF-Klassifikators}

Die dritte Methode setzt auf Ebene des RF-Klassifikators an. Die Probleme bei der Klassifikation von Daten mit unbalancierten Referenzdatensätzen treten auf, weil der RF-Klassifikator die Gesamtgenauigkeit maximiert. Eine Fehlklassifikation einer seltenen Klasse hat daher eine geringere Auswirkung auf den Fehler als die Fehlklassifikation einer häufigen Klasse. Durch die Einführung einer Gewichtung der Klassen kann dieser Effekt reduziert werden, wie Chen et al. (2004) zeigten.

Die genannten Maßnahmen erhöhen die Klassifikationsgenauigkeit. Daher kann davon ausgegangen werden, dass der in der vorliegenden Arbeit erzielte Klassifikationsfehler vor allem für die Klassen, die bisher im Referenzdatensatz unterrepräsentiert waren noch deutlich verringert werden kann.

Für die weitere Entwicklung eines Stichprobendesigns zur Erfassung der Referenzdaten im Kontext des Waldmonitorings muss außerdem beachtet werden, dass das Design kompatibel zu den terrestrischen Inventuren sein sollte, da häufig kombinierte Verfahren angewendet werden. Hier gibt es noch erheblichen Forschungsbedarf zu Stichprobenverfahren, die den teilweise widersprüchlichen Anforderungen des Modelltrainings, der Modellvalidierung und der terrestrischen Waldinventur gerecht werden können.

\subsection{Kartierung und Schätzung der Waldfläche im Rahmen internationaler Umweltabkommen}

Zielsetzung der Bildklassifikation war die Schätzung der Waldfläche und die Beschreibung der räumlichen Waldverteilung in Form einer Waldkarte. Die Ergebnisse aus den beiden Untersuchungsgebieten in Costa Rica zeigen, dass das entwickelte Verfahren prinzipiell geeignet ist, Waldkarten für unterschiedliche tropische Waldgebiete zu erstellen, welche einer zuvor festgelegten Walddefinition entsprechen. Insofern kann das Verfahren dazu beitragen die Transparenz von Waldkarten und den damit verbundenen Waldflächen- und Biomasseschätzungen zu erhöhen.

Die Hoffnung, durch die hohe zeitliche Auflösung des RapidEye-Systems wolkenfreie Aufnahmen auch in den Tropen zu erhalten, erfüllte sich nicht. Allerdings war dies auch darin begründet, das die RapidEye-Sensoren erst im Februar 2009 ihren operationalen Betrieb aufgenommen haben und daher bei der Bestellung der Bilder Ende 2009/Anfang 2010 keine 
Archivaufnahmen vorlagen. Deshalb mussten neue Aufnahmen in Auftrag gegeben werden (Tasking), welche jedoch im angesetzten Zeitraum nicht wolkenfrei geliefert werden konnten. Eine aktuelle Abfrage des RapidEye-Archivs zeigt, dass mittlerweile in Osa für die vier RapidEye-Tiles durchschnittlich 42 und in Guanacaste für die acht Tiles durchschnittlich 69 RapidEye-Aufnahmen pro Tile vorliegen (Stand: 17.Januar 2013). Allerdings ist die Qualität sehr unterschiedlich. Wolkenfreie Aufnahmen des gesamten Untersuchungsgebietes gibt es nach wie vor nicht.

Aufgrund der Bewölkung konnten in beiden Untersuchungsgebieten keine vollflächigen Waldkarten erstellt werden. Die Ausweisung von Flächen mit der Klasse „Keine Information“ ist die einzige konsistente Möglichkeit, Wolken und Wolkenschatten korrekt in den Landbedeckungskarten abzubilden. Bei der Ermittlung der Waldfläche und der Erstellung der Waldkarte führt das jedoch zu Problemen, da nicht klar ist welche Landnutzungsklassen unter den Wolken zu finden sind. Eine Möglichkeit, die in der vorliegenden Arbeit genutzt wurde, die Waldfläche trotzdem zu schätzen, ist die Extraploation des in den wolkenfreien Bildbereichen berechneten Waldanteils auf das gesamte Untersuchungsgebiet. Dieses Verfahren wird unter anderem auch für die Waldflächenschätzung der FRA 2010 in Indonesien angewendet (FRA, 2010a). Dabei wird die Grundannahme getroffen, dass die Verteilung der Landnutzungsklassen unter den Wolken identisch zur Verteilung in den wolkenfreien Gebieten, oder anders formuliert, die Verteilung der Wolken unabhängig von der Verteilung der Landnutzungsklassen ist. Für das Untersuchungsgebiet Osa muss jedoch bezweifelt werden, dass diese Annahme zutrifft. Die Wolken konzentrieren sich im Nordwesten des Untersuchungsgebietes, wo überwiegend Melina-, Teak-, Bananen- und Ölpalmenplantagen zu finden sind (vergl. Abbildung 32). Der südliche Teil des Untersuchungsgebietes, der von größeren Waldflächen dominiert wird, ist fast vollständig wolkenfrei. Daher muss angenommen werden, dass die Waldfläche überschätzt wird, wenn man die Verteilung der Landnutzung in den wolkenfreien Bereichen auch für die von Wolken bedeckten annimmt.

In Fällen, in denen die Grundannahme der Unabhängigkeit zwischen Wolken und Landnutzungsverteilung nicht gegeben ist oder wenn LSM berechnet werden sollen, muss eine Möglichkeit gefunden werden trotz der Wolken eine lückenlose Waldkarte zu erstellen. Eine mögliche Lösung des Problems ist die multitemporale Auswertung eines Gebietes anhand sehr dichter Zeitreihen. Durch die Konstellation der 5 Satelliten des RapidEye-Systems kann theoretisch eine Aufnahme pro Tag erfolgen. Außerdem gibt es für die meisten Gebiete mittlerweile große Mengen an Archivdaten. Durch die kombinierte Auswertung mehrerer Aufnahmen des selben Gebietes wird die Wahrscheinlichkeit erhört, dass alle Bildbereiche in 
mindestens einem Bild wolkenfrei sind. Die kompakten Wolken könnten, basierend auf der Level-I Karte, maskiert werden, da diese bereits gut erkannt werden. Für die Detektion von Dunst und Schleierwolken müssten jedoch verbesserte Algorithmen entwickelt werden. Der modulare Aufbau des ForestEye-Prozessors bietet hierfür eine hervorragende Grundlage. Im Anschluss der Klassifikation würden dann die Karten kombiniert und Bereiche mit der Klasse „keine Information“ mit den Kartenwerten aus den anderen Bildern gefüllt.

Neben der Schätzung der Waldfläche ist es besonders im Rahmen politischer Prozesse wichtig, die Genauigkeit der Schätzung zu ermitteln. Für die Verwendung der Waldflächenschätzung im Rahmen der Umweltschutzabkommen muss daher neben der Flächenschätzung auch ein Konfidenzintervall angegeben werden. McRoberts (2010) zeigt sowohl designals auch modellbasierte Ansätze zur Schätzung des Konfindenzintervalls und des Bias einer binären Landbedeckungskarte. Dieses Konzept wurde später auf drei Klassen erweitert (McRoberts, 2011), wobei die Fehlermatrizen für die Berechnung des Konfidenzintervalls und des Bias verwendet wurden. In der vorliegenden Arbeit wurde die Validierung der erstellten Landkarten auf die Level-I Karten beschränkt. Dies ist darin begründet, dass für die Validierung Punkte als Bezugsgröße verwendet wurden. Für die Zuordnung der LevelII Klassen zu einem Validierungspunkt wird jedoch eine Referenzfläche benötigt. Das hat zur Folge, dass ein Punkt während der Bildinterpretation nicht eindeutig einer der Level-II Klassen zugeordnet werden kann, ohne alle Objekte der Referenzfläche zu kartieren, um z. B. die Kronenüberschirmung zu bestimmen. Neben dem großen Aufwand der mit einem solchen Vorgehen verbunden ist, stellen sich auch einige theoretische Fragen in Bezug auf das Probeflächendesign für die Validierung: „Auf welcher Ebene der Klassifikationshierachie sollen die Polygone definiert werden? Wie können diese Polygone auf einer anderen Ebene mit anderen räumlichen Einheiten verwendet werden? "(Stehman \& Czaplewski, 1998). Eindeutige Antworten auf diese Fragen bei der Validierung von hierarchischen Klassifikationen gibt es bisher nicht und auch hier besteht noch erheblicher Forschungsbedarf. In der vorliegenden Arbeit kann aber davon ausgegangen werden, dass der Level-I Fehler als konservativer Schätzer für die Kartengenauigkeit der Level-II und III Karten verwendet werden kann, da die Anzahl der Klassen von Level-I nach Level-III abnimmt und somit auch weniger Verwechslungen auftreten können (Ioka \& Koda, 1986; Walker et al., 2010). Das bedeutet, dass die Genauigkeit der Waldflächenschätzung zwar unbekannt, jedoch höher als die der Level-I Karten ist. Diese Annahme entspricht dem Prinzip der konservativen Schätzung, wie es in den internationalen Umweltabkommen üblich ist. 

Teil V.

Schlußbetrachtung 



\section{Möglichkeiten und Grenzen der fernerkundlichen Waldflächenerfassung}

Die Anforderungen an die Waldinventur verändern sich mit den Ansprüchen der Gesellschaft an den Wald. Nachdem dieser viele Jahrhunderte in seiner Funktion als Holzlieferant im Vordergrund stand und somit die Erfassung der Vorräte und Zuwächse höchste Priorität hatte, treten jetzt zunehmend andere Waldfunktionen, wie der Klima- und Artenschutz, in den Fokus der öffentlichen Aufmerksamkeit. Außerdem ändern sich die Rahmenbedingungen für die Inventuren insofern, als Informationen nicht mehr nur lokal auf Betriebsebene für die Planung der Bewirtschaftung, sondern im Zusammenhang mit internationalen Abkommen und den sich daraus ergebenden Berichtspflichten auf nationaler bzw. internationaler Ebene benötigt werden. Für den Erfolg der internationalen Umweltabkommen ist es daher wichtig, dass die Inventuren transparenten Regeln folgen und somit konsistente Informationen liefern, die einen Vergleich auf internationaler Ebene ermöglichen. Konsequenterweise müssen deshalb bei der Erfassung der Waldflächen nachvollziehbare und eindeutig definierte Kriterien zur Unterscheidung von Wald- und Nicht-Waldflächen angewendet werden.

Ziel der vorliegenden Arbeit war es, methodische und technische Aspekte der fernerkundlichen Erfassung der Waldfläche und deren Fragmentierung zu untersuchen. Die Ergebnisse zeigen theoretisch, empirisch und in der praktischen Anwendung, dass eine Vielzahl von Faktoren die Erfassung der Waldfläche beeinflusst. Zentral ist dabei die Walddefinition. Die empirische Simulation untersuchte zwei Kriterien der Walddefinition: den Überschirmungsschwellenwert und die Referenzflächengröße und zeigte spezifische Wechselwirkungen zwischen beiden auf. Diese Wechselwirkungen konnten teilweise theoretisch anhand eines geometrischen Waldrandmodells erklärt werden, aus dem sich folgende Schlussfolgerung ergab: Wenn die Referenzflächengröße nicht explizit definiert wird und sich der Schwellenwerte für die Kronenüberschirmung signifikant von $50 \%$ unterscheidet, sind die Waldflächenschätzungen mit starken Unsicherheiten behaftet. Beides, Schwellenwerte, die deutlich von $50 \%$ abweichen und das Fehlen einer Definition für die Referenzfläche sind momentan Grundlage international verwendeter Walddefinitionen. Die Festlegung einer Referenzfläche könnte die Unsicherheiten der aktuellen Waldflächenschätzung somit erheblich reduzieren. 
Aufgrund der besonderen Bedeutung der Walddefinition für die Waldflächenerfassung wurde im dritten Teil der Arbeit ein Verfahren entwickelt, das es ermöglicht, spezifische Kriterien der Walddefinition in die Auswertung von Satellitenbildern zu integrieren und somit konsistente Waldkarten zu erstellen. Beispielgebend waren die Kriterien der FAOWalddefinition. Dabei handelt es sich um eine Landnutzungsdefinition. Grundsätzlich kann in Fernerkundungsdaten nur die Landbedeckung beobachtet werden, so dass die direkte Unterscheidung von Wald- und Nicht-Waldflächen nach der FAO-Definition in einem Satellitenbild nicht möglich ist. Um eine Landbedeckungskarte in eine FAO-Landnutzungskarte zu überführen, muss die Landbedeckung in einem räumlichen und zeitlichen Kontext betrachtet werden. So kann zum Beispiel für die Landbedeckungsklasse Baumkrone nicht entschieden werden, ob es sich um einen Einzelbaum oder einen Wald handelt, wenn der räumliche Kontext, also die Landbedeckung in den angrenzenden Bereichen, nicht berücksichtigt wird. Durch die Implementierung eines hierarchischen Klassifikationschlüssels in Kombination mit dem Referenzfüchenverfahren konnte ein Ansatz entwickelt werden, der diesen räumlichen Zusammenhang berücksichtigt. Die Integration zeitlicher Kontexte konnte bisher nicht erfolgen. So ist es bspw. noch nicht möglich, Aussagen darüber zu treffen, ob eine bestimmte Fläche nach einem Brand innerhalb einer Frist wieder eine bestimmte Höhe oder Kronenüberschirmung erreichen wird. Die Verwendung solcher „potentieller“ Kriterien ist politisch begründbar, erhöht aber immer die Unsicherheit der Waldflächenschätzung. Daher ist es aus wissenschaftlich-rationaler Sicht fragwürdig, ob solche Kriterien im Rahmen der internationalen Umweltabkommen verwendet werden sollten.

Neben der Waldfläche wurde auch die Beschreibung der Waldfragmentierung mittels LSM untersucht. Dabei zeigte sich, dass die LSM prinzipiell als Indikatoren für die Waldfragmentierung geeignet sind und mit dem vorgestellte Verfahren auch auf Basis von dreidimensionalen Landschaftsmodellen erfolgen kann. Insofern kann das Verfahren und die dafür entwickelte Software als Werkzeug für weitere wissenschaftliche Untersuchungen dienen. Solche Untersuchungen könnten z. B. der Frage nachgehen, ob durch die Berücksichtigung der dreidimensionalen Landschaftsmodelle die Aussagekraft der LSM als Biodiversitätsindikator verbessert werden kann.

Es ist abzusehen, dass sich die Technologie und Verfügbarkeit von Fernerkundungsdaten weiter verbessern wird und dadurch zunehmend für die Erfassung natürlicher Ressourcen eingesetzt werden kann. Da mit Fernerkundungsdaten nur sehr wenige Variablen direkt beobachtet werden können, finden häufig jene Modelle Verwendung, die den Zusammenhang der am Sensor gemessenen Reflexion und einer forstliche Zielgröße beschreiben. Zur 
Parametrisierung dieser Modelle werden Informationen aus terrestrischen Inventuren benötigt. Da zudem viele forstliche Größen ausschließlich im Feld aufgenommen werden können, ist davon auszugehen, dass die terrestrische Waldinventur weiterhin ein fester Bestandteil des Waldmonitorings bleiben wird. Eine Kombination der terrestrischen und fernerkundlichen Erfassung scheint momentan am vorteilhaftesten. Das in der vorliegenden Arbeit verwendete Referenzflächenverfahren eignet sich für den Einsatz in kombinierten Monitoringverfahren. In Bezug auf die Implementierung kombinierter Inventuren gibt es aber noch erheblichen Forschungsbedarf, besonders bei der Entwicklung von Stichprobenverfahren, die sich sowohl für die Schätzung der Zielgrößen einer terrestrischen Waldinventur als auch für die Parametrisierung der Klassifikationsmodelle zur Auswertung der Fernerkundungsdaten eignen.

In letzter Zeit gibt es vermehrt Ansätze Ökosystem-Dienstleistungen (engl. environmental services) auf Landschaftsebene zu erfassen. Dies wird unter anderem am Motto „Living Landscapes" des 6. Forest Days, einer Veranstaltung im Rahmenprogramm der 18. Konferenz der Vertragstaaten des UNFCCC, deutlich. Bei diesem Treffen internationaler Forstexperten stand die Wechselwirkung zwischen Forst- und Landwirtschaft als landschaftsprägende Landnutzungen im Vordergrund. Das in der vorliegenden Arbeit verwendete Referenzflächenverfahren liefert, neben den Landbedeckungs-, Landnutzungs-, und Waldkarten, als Nebenprodukt auch Kronendichtekarten. Diese können als Grundlage für die kontinuierliche Erfassung der Kronenüberschirmung auf Landschaftsebene, unabhängig von diskreten Klassen, verwendet werden. Hier sollten weitere Untersuchungen zum Zusammenhang zwischen der Kronendichte und holziger Biomasse erfolgen, um zu untersuchen inwiefern die Kronendichtekarten die Verteilung der holzigen Biomasse auf Landschaftsebene beschreiben können. Sollte dies möglich sein, könnte zumindest aus wissenschaftlich-rationaler Sicht eine Einteilung der Landschaft in verschiedene Landnutzungsklassen hinfällig sein, da es für die Klimawirkung unerheblich ist, wo Treibhausgas gespeichert bzw. emittiert wird.

Momentan ist nicht absehbar, ob es gelingt, im Kontext der internationalen Umweltabkommen, konsistente und transparente Methoden zur Erfassung der Waldfläche zu vereinbaren. Die Ergebnisse dieser Arbeit können dazu beitragen, einige für die Standardisierung relevante Aspekte besser zu verstehen. Auch wenn die vollständige Standardisierung der Waldflächenerfassung momentan nicht in Reichweite ist, soll das abschließende und von der Geschichte widerlegte 200 Jahre alte Zitat dafür stehen, die Hoffnung nicht aufzugeben: 
„Da in Deutschland wahrscheinlich niemals ein allgemeines Maas eingeführt werden wird, und die Umstände sehr oft nicht einmal zulassen, daß nur in manchem kleinen Teilen von Deutschland für alle Flächenmessungen ein gleiches Maas vorgeschrieben werden kann; so ist die Auswahl eines allgemeinen Maases für die Waldmessung mit keinen Schwierigkeiten verknüpft. Sie kann ganz willkührlich geschehen. Doch würde ich rathen, ein solches Maas zu wählen, das zwischen allen im Land gebräuchlichen Maasen das Mittel hält, damit keine allzu große Differenz zwischen dem neuen Waldmaase und den älteren Flächenmaasen statt finde." (Hartig, 1813) 
Teil VI.

\section{Literatur}





\section{Literaturverzeichnis}

Atkinson, P. \& Curran, P. (1995). Defining an optimal size of support for remote sensing investigations. IEEE Transactions on Geoscience and Remote Sensing, 33(3), 768-776.

AWG-LCA (2010). Policy approaches and positive incentives on issues relating to reducing emissions from deforestation and forest degradation in developing countries; and the role of conservation, sustainable management of forests and enhancement of forest carbon stocks in developing countries. Technical Report FCCC/AWGLCA/2010/L.7, Ad hoc Working Group on Long-term Cooperative Action under the Convention, Geneva (Switzerland).

Axelsson, A.-L., Ståhl, G., Söderberg, U., Petersson, H., Fridman, J. \& Lundström, A. (2010). Sweden. In E. Tomppo, T. Gschwantner, M. Lawrence, \& R. McRoberts (Eds.), National Forest Inventories - Pathways for Common Reporting (pp. 541-553). Springer.

Bartholomé, E. \& Belward, A. S. (2005). GLC2000: a new approach to global land cover mapping from Earth observation data. International Journal of Remote Sensing, 26(9), 1959-1977.

Benson, B. \& MacKenzie, M. (1995). Effects of sensor spatial resolution on landscape structure parameters. Landscape Ecology, 10(2), 113-120.

Bossard, M., Feranec, J. \& Otahel, J. (2000). CORINE land cover technical guide: Addendum 2000. Technical report 40, European Environment Agency, Copenhagen.

Boulesteix, A.-L., Janitza, S., Kruppa, J. \& König, I. R. (2012). Overview of Random Forest Methodology and Practical Guidance with Emphasis on Computational Biology and Bioinformatics. Technical Report 129, Institut für Statistik, Ludwig-MaximiliansUniversität, München.

Breiman, L. (2001). Random Forests. Machine Learning, 45(1), 5-32.

Breiman, L. \& Cutler, A. (2012). Random forest manual. [online] http://www.stat . berkeley.edu/?breiman/RandomForests/cchome.htm. last visited 12.10. 2012. 
Breiman, L., Friedman, J., Stone, C. \& Olshen, R. (1984). Classification and regression trees. Chapman \& Hall/CRC.

Brändli, U.-B., Brassel, P., Duc, P., Keller, M., Köhl, M., Herold, A., Kaufmann, E., Lischke, H., Paschedag, P., Schnellbächer, H.-J., Schwyzer, A., Stierlin, H. R., Strobel, T., Traub, B., Ulmer, U. \& Zinggeler, J. (2001). Swiss National Forest Inventory: Methods and Models of the Second Assessment. Birmersdorf, Swiss: Federal Research Institute WSL.

Butchart, S. H. M., Walpole, M., Collen, B., van Strien, A., Scharlemann, J. P. W., Almond, R. E. a., Baillie, J. E. M., Bomhard, B., Brown, C., Bruno, J., Carpenter, K. E., Carr, G. M., Chanson, J., Chenery, A. M., Csirke, J., Davidson, N. C., Dentener, F., Foster, M., Galli, A., Galloway, J. N., Genovesi, P., Gregory, R. D., Hockings, M., Kapos, V., Lamarque, J.-F., Leverington, F., Loh, J., McGeoch, M. a., McRae, L., Minasyan, A., Hernández Morcillo, M., Oldfield, T. E. E., Pauly, D., Quader, S., Revenga, C., Sauer, J. R., Skolnik, B., Spear, D., Stanwell-Smith, D., Stuart, S. N., Symes, A., Tierney, M., Tyrrell, T. D., Vié, J.-C. \& Watson, R. (2010). Global biodiversity: indicators of recent declines. Science (New York, N.Y.), 328(5982), 1164-8.

Campbell, J. B. (2002). Introduction to Remote Sensing. Guilford Press, 3rd edition.

Chang, F., Chen, C. \& Lu, C. (2004). A linear-time component-labeling algorithm using contour tracing technique. Computer Vision and Image Understanding, 93(2), 206-220.

Chen, C., Liaw, A. \& Breiman, L. (2004). Using random forest to learn imbalanced data. Statistics Technical Report 666, Department of Statistics, UC Berkeley, Berkeley.

Chen, D. \& Wei, H. (2009). The effect of spatial autocorrelation and class proportion on the accuracy measures from different sampling designs. ISPRS Journal of Photogrammetry and Remote Sensing, 64(2), 140-150.

Cicvo, D. (1989). Topographic normalization of Landsat Thematic Mapper digital imagery. Photogrammetric Engineering and Remote Sensing, 55, 1303-1309.

Clausi, D. A. (2002). An analysis of co-occurrence texture statistics as a function of grey level quantization. Canadian Journal of remote sensing, 28(1), 45-62.

Colson, F., Bogaert, J., Carneiro, A., Nelson, B., Pinagé, E. \& Ceulemans, R. (2009). The influence of forest definition on landscape fragmentation assessment in Rondônia, Brazil. 
Ecological Indicators, 9(6), 1163-1168.

Congalton, R. (1988). A comparison of sampling schemes used in generating error matrices for assessing the accuracy of maps generated from remotely sensed data. Photogrammetric Engineering and Remote Sensing, 54(5), 593-600.

Conners, R., Trivedi, M. \& Harlow, C. (1984). Segmentation of a high-resolution urban scene using texture operators. Computer Vision, Graphics, and Image Processing, 25(3), 273-310.

De Fries, R. S., Hansen, M., Townshend, J. R. G. \& Sohlberg, R. (1998). Global land cover classifications at $8 \mathrm{~km}$ spatial resolution: The use of training data derived from landsat imagery in decision tree classifiers. International Journal of Remote Sensing, 19(16), $3141-3168$.

DeFries, R., Achard, F., Brown, S., Herold, M., Murdiyarso, D.and Schlamadinger, B. \& Souza, C. D. (2006). Developing Countries: Considerations for Monitoring and Measuring. Report 46 /26, Global Terrestrial Observing System (GTOS) / GOFC-GOLD, www.fao.org/gtos/pubs.html.

DeFries, R., Hansen, M., Townshend, J., Janetos, A. \& Loveland, T. (2000). A new global 1$\mathrm{km}$ dataset of percentage tree cover derived from remote sensing. Global Change Biology, $6(2), 247-254$.

DeFries, R. \& Townshend, J. (1994). Ndvi-derived land cover classifications at a global scale. International Journal of Remote Sensing, 15(17), 3567-3586.

Dorner, B., Lertzman, K. \& Fall, J. (2002). Landscape pattern in topographically complex landscapes: issues and techniques for analysis. Landscape Ecology, 17(8), 729-743.

Dupret, G. \& Koda, M. (2001). Bootstrap re-sampling for unbalanced data in supervised learning. European Journal of Operational Research, 134(1), 141-156.

El Hajj, M., Bégué, A., Lafrance, B., Hagolle, O., Dedieu, G. \& Rumeau, M. (2008). Relative Radiometric Normalization and Atmospheric Correction of a SPOT 5 Time Series. Sensors, 8(4), 2774-2791.

Eysn, L., Hollaus, M., Schadauer, K. \& Pfeifer, N. (2012). Forest Delineation Based on Airborne LIDAR Data. Remote Sensing, 4(3), 762-783. 
Fabian, P. (2002). Leben im Treibhaus: unser Klimasystem - und was wir daraus machen. Springer Verlag.

Falkowski, M., Evans, J., Martinuzzi, S., Gessler, P. \& Hudak, A. (2009). Characterizing forest succession with lidar data: An evaluation for the Inland Northwest, USA. Remote Sensing of Environment, 113(5), 946-956.

Filella, I. \& Penuelas, J. (1994). The red edge position and shape as indicators of plant chlorophyll content, biomass and hydric status. International Journal of Remote Sensing, 15(7), 1459-1470.

Fischbach von, C. (1886). Lehrbuch der Forstwissenschaft für Forstmänner und Waldbesitzer. J. Springer.

Foody, G. M. \& Mathur, A. (2006). The use of small training sets containing mixed pixels for accurate hard image classification: Training on mixed spectral responses for classification by a SVM. Remote Sensing of Environment, 103(2), 179-189.

Forman, R. (1995a). Land mosaics: the ecology of landscapes and regions. Cambridge Univ Press.

Forman, R. (1995b). Some general principles of landscape and regional ecology. Landscape Ecology, 10(3), 133-142.

FRA (2010a). Global Forest Resources Assessment: Country Report Indonesia. Technical Report 2010/095, Food and Agriculture Organization of the United Nations, Forestry Department, Rome.

FRA (2010b). Global Forest Resources Assessment: Main Report. Foresty Paper 163, Food and Agriculture Organization of the United Nations, Forestry Department, Rome.

Friedl, M., McIver, D., Hodges, J., Zhang, X., Muchoney, D., Strahler, A., Woodcock, C., Gopal, S., Schneider, A., Cooper, A. et al. (2002). Global land cover mapping from MODIS: algorithms and early results. Remote Sensing of Environment, 83(1), 287-302.

Frohn, R. \& Hao, Y. (2006). Landscape metric performance in analyzing two decades of deforestation in the Amazon Basin of Rondonia, Brazil. Remote sensing of environment, $100(2), 237-251$. 
Förster, M., Schuster, C., Sonnenschein, R., Bahls, A. \& Kleinnschmit, B. (2011). Möglichkeiten der Erfassung von Landbedeckung und Vegetationsgesellschaften mittels RapidEyeDaten. In E. Borg \& H. Daedelow (Eds.), RapidEye Science Archive Erste Ergebnisse, 3. Resa Workshop Neustrelitz: DLR.

Gadow, K. v. (2005). Forsteinrichtung. Universitätsdruck Göttingen.

Gislason, P. O., Benediktsson, J. A. \& Sveinsson, J. R. (2006). Random forests for land cover classification. Pattern Recognition Letters, 27(4), 294-300.

Gitelson, A. \& Merzlyak, M. (1997). Remote estimation of chlorophyll content in higher plant leaves. International Journal of Remote Sensing, 18(12), 2691-2697.

Gitelson, A., Viña, A., Ciganda, V., Rundquist, D. C. \& Arkebauer, T. (2005). Remote estimation of canopy chlorophyll content in crops. Geophysical Research Letters, 32(8).

Gneiting, T. \& Schlather, M. (2004). Stochastic models that separate fractal dimension and the Hurst effect. SIAM review, (pp. 269-282).

Goetz, S. J., Wright, R. K., Smith, A. J., Zinecker, E. \& Schaub, E. (2003). IKONOS imagery for resource management: Tree cover, impervious surfaces, and riparian buffer analyses in the mid-Atlantic region. Remote Sensing of Environment, 88(1-2), 195-208.

Gotway, C. \& Young, L. (2002). Combining incompatible spatial data. Journal of the American Statistical Association, 97(458), 632-648.

GRASS Development Team (2011). Geographic Resources Analysis Support System (GRASS GIS) Software. Open Source Geospatial Foundation.

Gregoire, T. \& Valentine, H. (2008). Sampling strategies for natural resources and the environment, volume 1. CRC Press.

Grieser, J., Gommes, R. \& Bernardi, M. (2006). New locclim - the local climate estimator of fao. In Geophys Res Abstr, volume 8.

Guariguata, M. R., Nasi, R. \& Kanninen, M. (2009). Forest degradation: it is not a matter of new definitions. Conservation Letters, 2(6), 286-287.

Gustafson, E. \& Parker, G. (1992). Relationships between landcover proportion and indices 
of landscape spatial pattern. Landscape Ecology, 7(2), 101-110.

Guyon, I. \& Elisseeff, A. (2003). An introduction to variable and feature selection. The Journal of Machine Learning Research, 3, 1157-1182.

Hadjimitsis, D. G., Clayton, C. R. I. \& Hope, V. S. (2004). An assessment of the effectiveness of atmospheric correction algorithms through the remote sensing of some reservoirs. International Journal of Remote Sensing, 25(18), 3651-3674.

Hansen, M. \& DeFries, R. (2000). Global land cover classification at $1 \mathrm{~km}$ spatial resolution using a classification tree approach. International Journal of Remote Sensing, 21(6 \& 7), $1331-1364$.

Hansen, M., DeFries, R., Townshed, J., Caroll, M., Dimiceli, C. \& Sohlberg, R. (2003). Vegetation continous fields mod44b. University of Maryland, Version 3.0.

Hansen, M., DeFries, R., Townshend, J., Marufu, L. \& Sohlberg, R. (2002). Development of a MODIS tree cover validation data set for western province, zambia. Remote Sensing of Environment, 83(12), 320-335.

Hansen, M., Townshend, J., DeFries, R. \& Carroll, M. (2005). Estimation of tree cover using MODIS data at global, continental and regional/local scales. International Journal of Remote Sensing, 26(19), 4359-4380.

Haralick, R. M., Shanmugam, K. \& Dinstein, I. (1973). Textural features for image classification. IEEE Transactions on Systems, Man and Cybernetics, 3(6), 610-621.

Harrison, S. \& Bruna, E. (1999). Habitat fragmentation and large-scale conservation: what do we know for sure? Ecography, 22(3), 225-232.

Hartig, G. L. (1813). Anweisung zur Taxation und Beschreibung der Forste - Erster theoretischer Theil nebst illuminierten Forstkarten, Schema und mehreren Tabellen. Gießen, Darmstadt: Georg Friedrich Heuer. Dritte, abermals verbesserte und vermehrte Auflage.

Hasel, K. \& Schwartz, E. (2006). Forstgeschichte. Verlag Kessel, 3 edition.

Herold, M., Mayaux, P., Woodcock, C., Baccini, A. \& Schmullius, C. (2008). Some challenges in global land cover mapping: An assessment of agreement and accuracy in existing $1 \mathrm{~km}$ datasets. Remote Sensing of Environment, 112(5), 2538-2556. 
Hoechstetter, S., Walz, U., Dang, L. \& Thinh, N. X. (2008). Effects of topography and surface roughness in analyses of landscape structure- a proposal to modify the existing set of landscape metrics. Landscape Online, 1, 1-14.

Holdridge, L. R. (1967). Life Zone Ecology. Tropical science center, San Jose, Costa Rica.

Holmgren, P., Clairs, T. \& Kasten, T. (2008). Role of satellite remote sensing in REDD. Working Paper 1, UN-REDD Programm.

Houghton, R., Butman, D., Bunn, A., Krankina, O., Schlesinger, P. \& Stone, T. (2007). Mapping russian forest biomass with data from satellites and forest inventories. Environmental Research Letters, 2(4), 045032.

IEEE (1990). Standard Glossary of Image Processing and Pattern Recognition Terminology. Standard 610.4, Institute of Electrical and Electronics Engineers.

Immitzer, M., Atzberger, C. \& Koukal, T. (2012). Tree species classification with random forest using very high spatial resolution 8-band worldview-2 satellite data. Remote Sensing, 4(9), 2661-2693.

Ioka, M. \& Koda, M. (1986). Performance of LANDSAT-5 TM data in land-cover classification? International Journal of Remote Sensing, 7(12), 1715-1728.

Janzen, D. (1988). Tropical dry forests. The most endangered major tropical ecosystem. In E. Wilson (Ed.), Biodiversity (pp. 130-137). Washington DC: National Academy of Sciences / Smithsonian Institution.

Jelinski, D. \& Wu, J. (1996). The modifiable areal unit problem and implications for landscape ecology. Landscape Ecology, 11(3), 129-140.

Jenness, J. S. (2004). Calculating landscape surface area from digital elevation models. Wildlife Society Bulletin, 32(3), 829-839.

Kanninen, M., Murdiyarso, D., Seymour, F., Angelsen, A., Wunder, S. \& German, L. (2007). Do trees grow on money: The implications of deforestation research for policies to promote $R E D D$. Number 4 in Forest Prespectives. Center for International Forestry Research (CIFOR).

Karjalainen, T., Richards, G., Hernandez, T., Kainja, s., Lawson, G., Liu, S., Prisley, S., 
Pardo, J. I. A., Birdsey, R., Bohem, M., Daka, J., Kobayashi, S., Lund, H. G., Michalak, R. \& Takahashi, M. (2003). Definitions and methodological options to inventory emissions from direct human-induced degradation of forests and devegetation of other vegetation types. IGES. IPCC.

Keller, M. (2005). Schweizerisches Landesforstinventar. Anleitung für die Feldaufnahmen der Erhebung 2004-200\%. Birmersdorf: Eidgenösische Forschungsanstalt WSL.

Kleinn, C. (1991). Zum Waldbegriff in Forstlichen Großrauminventuren (Conceptos forestales en inventarios forestales en grandes espacios). Allgemeine Forst und Jagdzeitung, $162,201-210$.

Kleinn, C. (1992). On the Comparability of Forest Inventory Results. In G. Wood \& B. Turner (Eds.), Proceedings of the international IUFRO Conference on integrated Forest Management over Space and Time (pp. 278-285). Canberra, Australia.

Kleinn, C. (2001). A cautionary note on the minimum crown cover criterion in forest definitions. Canadian Journal of Forest Research, 31(2), 350-356.

Kleinn, C., Corrales, L. \& Morales, D. (2002). Forest Area in Costa Rica: A Comparative Study of Tropical Forest Cover Estimates over Time. Environmental Monitoring and Assessment, 73(1), 17-40.

Kleinn, C., Ramirez, C., Holmgren, P., Valverde, S. \& Chavez, G. (2005). A national forest resources assessment for costa rica based on low intensity sampling. Forest Ecology and Management, 210(1-3), 9-23.

Ko, D., Bristow, N., Greenwood, D. \& Weisberg, P. (2009). Canopy cover estimation in semiarid woodlands: comparison of field-based and remote sensing methods. Forest Science, $55(2), 132-141$.

Korhonen, L., Korhonen, K., Rautiainen, M. \& Stenberg, P. (2006). Estimation of forest canopy cover: a comparison of field measurement techniques. Silva Fennica, 40(4), 577588.

Krummel, J., Gardner, R., Sugihara, G., O’Neill, R. V. \& Coleman, P. R. (1987). Landscape patterns in a disturbed environment. Oikos, 48, 321-324.

Kupfer, J., Malanson, G. \& Franklin, S. (2006). Not seeing the ocean for the islands: the 
mediating influence of matrix-based processes on forest fragmentation effects. Global Ecology and Biogeography, 15(1), 8-20.

Lanz, A. (2005). Stichproben am Waldrand - Probleme und neue Lösungsansätze. In U. Wunn (Ed.), Deutscher Verband Forstlicher Forschungsanstalten 17. Tagungsband Freiburg: Forschungsanstalt für Waldökologie und Forstwissenschaft Rheinland-Pfalz.

Laurance, W., Camargo, J., Luizão, R., Laurance, S., Pimm, S., Bruna, E., Stouffer, P., Bruce Williamson, G., Benítez-Malvido, J., Vasconcelos, H., K.S., H., Zartman, C., Boyle, S., K., D. R., Andrade, A. \& Lovejoy, T. (2010). The fate of Amazonian forest fragments: A 32-year investigation. Biological Conservation.

Laurance, W. \& Peres, C. (2006). Emerging threats to tropical forests. University of Chicago Press.

Leitão, A., Miller, J., Ahern, J. \& McGardigal, K. (2006). Measuring landscapes: a planner's handbook. Island Presss.

Lemmon, P. (1956). A spherical densiometer for estimating forest overstory density. Forest Science, 2(4), 314-320.

Li, H. \& Wu, J. (2004). Use and misuse of landscape indices. Landscape Ecology, 19(4), 389-399.

Liu, Y., Chawla, N. V., Harper, M. P., Shriberg, E. \& Stolcke, A. (2006). A study in machine learning from imbalanced data for sentence boundary detection in speech. Computer Speech \&3 Language, 20(4), 468-494.

Loveland, T., Reed, B., Brown, J., Ohlen, D., Zhu, Z., Yang, L. \& Merchant, J. (2000). Development of a global land cover characteristics database and IGBP DISCover from $1 \mathrm{~km}$ AVHRR data. International Journal of Remote Sensing, 21(6-7), 1303-1330.

Lund, G. (2011). Definitions of Forest, Deforestation, Afforestation, and Reforestation. [online] http://home.comcast.net/ gyde/DEFpaper.htm. last visited 24.08.2011.

Lund, H. G. (2002). When Is a Forest Not a Forest? Journal of Forestry, 100(8), 21-28.

MacArthur, R. \& Wilson, E. (1967). The theory of island biogeography. Princeton Univ Press. 
Magdon, P. (2011). patch3d: Calculation of 3d patch metrics. [online] http://code.google . $\mathrm{com} / \mathrm{p} / \mathrm{patch} 3 \mathrm{~d} /$. last visited 20.1.2013.

Magdon, P., Fischer, C., Fuchs, H. \& Kleinn, C. (2013). Translating Criteria of International Forest Definitions into Remote Sensing Image Analysis. Remote Sensing of Environment. in review.

Magdon, P. \& Kleinn, C. (2012). Uncertainties of forest area estimates caused by the definition of minimum crown cover - a scale issue relevant to forest definitions. Environmental Monitoring and Assessment, (pp. 1-16). doi: 10.1007/s10661-012-2950-0.

Main-Knorn, M., Moisen, G., Healey, S., Keeton, W., Freeman, E. \& Hostert, P. (2011). Evaluating the remote sensing and inventory-based estimation of biomass in the western carpathians. Remote Sensing, 3(7), 1427-1446.

Mandelbrot, B. B. (1983). The Fractal Geometry of Nature. New York: Freeman.

Marceau, D. (1999). The scale issue in social and natural sciences. Canadian Journal of Remote Sensing, 25(4), 347-356.

Mareceau, D. J. \& Geoffrey, J. H. (1999). Remote sensing contributions to the scale issue. Canadian Journal of Remote Sensing, 25(4), 357-366.

Marshall, A., Jørgensbye, H., Rovero, F., Platts, P., White, P. \& Lovett, J. (2010). The species-area relationship and confounding variables in a threatened monkey community. American journal of primatology, 72(4), 325-336.

Martínez, S. \& Mollicone, D. (2012). From Land Cover to Land Use: A Methodology to Assess Land Use from Remote Sensing Data. Remote Sensing, 4(4), 1024-1045.

Marx, A. (2010). Erkennung von Borkenkäferbefall in Fichtenreinbeständen mit multitemporalen RapidEye Satellitenbildern und Datamining-Techniken. PhotogrammetrieFernerkundung-Geoinformation, 4, 243-252.

Mathys, L., Ginzler, C., Zimmermann, N. E., Brassel, P. \& Wildi, O. (2006). Sensitivity assessment on continuous landscape variables to classify a discrete forest area. Forest Ecology and Management, 229(1-3), 111-119.

Maurer, F. \& Rupp, H. (1974). Deutsche Wortgeschichte. Walter de Gruyter. 
McGarigal, K. \& Cushman, S. (2005). The gradient concept of landscape structure. In J. A. Wiens \& M. R. Moss (Eds.), Issues and Perspectives in Landscape ecology chapter 12, (pp. 112-127). Cambridge University Press.

McGarigal, K., Cushman, S., Neel, M. \& Ene, E. (2002). FRAGSTATS: spatial pattern analysis program for categorical maps. University of Massachusetts, Amherst, Massachusetts, USA.

McRoberts, R. E. (2010). Probability- and model-based approaches to inference for proportion forest using satellite imagery as ancillary data. Remote Sensing of Environment, 114(5), 1017-1025.

McRoberts, R. E. (2011). Satellite image-based maps: Scientific inference or pretty pictures? Remote Sensing of Environment, 115(2), 715-724.

Moser, D., Zechmeister, H. G., Plutzar, C., Sauberer, N., Wrbka, T. \& Grabherr, G. (2002). Landscape patch shape complexity as an effective measure for plant species richness in rural landscapes. Landscape Ecology, 17(7), 657-669.

Nelson, M., McRoberts, R., Holden, G. \& Bauer, M. (2009). Effects of satellite image spatial aggregation and resolution on estimates of forest land area. International Journal of Remote Sensing, 30(8), 1913-1940.

O’Neill, R., Hunsaker, C., Timmins, S., Jackson, B., Jones, K., Riitters, K. \& Wickham, J. (1996). Scale problems in reporting landscape pattern at the regional scale. Landscape Ecology, 11(3), 169-180.

Ozanne, C., Anhuf, D., Boulter, S., Keller, M., Kitching, R., Körner, C., Meinzer, F., Mitchell, A., Nakashizuka, T., Dias, P., Stork, N., Wright, S. \& Yoshimura, M. (2003). Biodiversity meets the atmosphere: a global view of forest canopies. Science, 301(5630), 183.

Pal, M. (2005). Random forest classifier for remote sensing classification. International Journal of Remote Sensing, 26(1), 217-222.

Pan, Y., Birdsey, R. A., Fang, J., Houghton, R., Kauppi, P. E., Kurz, W. A., Phillips, O. L., Shvidenko, A., Lewis, S. L., Canadell, J. G., Ciais, P., Jackson, R. B., Pacala, S. W., McGuire, A. D., Piao, S., Rautiainen, A., Sitch, S. \& Hayes, D. (2011). A large and persistent carbon sink in the world's forests. Science (New York, N.Y.), 333(6045), 
988-93.

Parkinson, C. \& Greenstone, R. (2000). EOS data products handbook. EOS Project Science Office, Greenbelt, Maryland. Vol. 2.

Pekkarinen, A., Reithmaier, L. \& Strobl, P. (2009). Pan-European forest/non-forest mapping with Landsat ETM+ and CORINE Land Cover 2000 data. ISPRS Journal of Photogrammetry and Remote Sensing, 64(2), 171-183.

Powell, S. L., Cohen, W. B., Healey, S. P., Kennedy, R. E., Moisen, G. G., Pierce, K. B. \& Ohmann, J. L. (2010). Quantification of live aboveground forest biomass dynamics with Landsat time-series and field inventory data: A comparison of empirical modeling approaches. Remote Sensing of Environment, 114(5), 1053-1068.

Putz, F. \& Redford, K. (2010). The Importance of Defining'Forest': Tropical Forest Degradation, Deforestation, Long-term Phase Shifts, and Further Transitions. Biotropica, 42(1), 10-20.

Putz, F. \& Sasaki, N. (2009). What is 'forest?' Response to Guariguata et al. Conservation Letters, 2(6), 288-289.

R Development Core Team (2011). R: A Language and Environment for Statistical Computing. R Foundation for Statistical Computing, Vienna, Austria.

RapidEye AG (2012). Satellite Imagery Product Specifications. Version 3.3, RapidEye AG, http://www.rapideye.net/upload/RE_Product_Specifications_ENG.pdf.

Riano, D., Chuvieco, E., Salas, J. \& Aguado, I. (2003). Assessment of different topographic corrections in landsat-tm data for mapping vegetation types (2003). Geoscience and Remote Sensing, IEEE Transactions on, 41(5), 1056-1061.

Rodriguéz, E., Morris, C. \& Belz, J. (2006). A global assessment of the srtm performance. Photogrammetric Engineering and Remote Sensing, 72(3), 249-260.

Rodriguez-Galiano, V., Ghimire, B., Rogan, J., Chica-Olmo, M. \& Rigol-Sanchez, J. (2012). An assessment of the effectiveness of a random forest classifier for land-cover classification. ISPRS Journal of Photogrammetry and Remote Sensing, 67, 93-104.

Running, S., Loveland, T. \& Pierce, L. (1994). A vegetation classification logic based on 
remote sensing for use in global biogeochemical models. AMBIO, 23, 77-77.

Rutledge, D. (2003). Landscape indices as measures of the effects of fragmentation: can pattern reflect process? Technical Report 98, Department of Conservation, Wellington.

Sasaki, N. \& Putz, F. (2009). Critical need for new definitions of"foreständ"forest degradationïn global climate change agreements. Conservation Letters, 2(5), 226-232.

Saunders, D., Hobbs, R. \& Margules, C. (1991). Biological consequences of ecosystem fragmentation: a review. Conservation biology, 5(1), 18-32.

Saura, S. \& Castro, S. (2007). Scaling functions for landscape pattern metrics derived from remotely sensed data: Are their subpixel estimates really accurate? ISPRS journal of photogrammetry and remote sensing, 62(3), 201-216.

SBSTA (2009). Methodologies guidance for activities relating to reducing emissions from deforestation and forest degradation and the role of conservation, sustainable management of forest and enhancement of forest carbon stocks in developing countries. Decision 4/CP.15. FCCC/SBSTA/2009/2, Subsidary Body for Scientific and Technological Advice.

Schabenberger, O. \& Gotway, C. (2005). Statistical Methods for Spatial Data Analysis. Chapman \& Hall /CRC.

Schlather, M. (2001). Simulation of stationary and isotropic random fields. R-News, 1, $18-20$.

Schmitz, F., Polley, H., Hennig, P., Dunger, K. \& Frank, S. (2008). Die zweite Bundeswaldinventur-BW $I^{2}$ Inventur- und Auswertungsmethoden; Zu den Bundeswaldinventuren 2001 bis 2002 und 1986 bis 1988. Arbeitsbericht 2008/1, Johann Heinrich von Thünen-Institut.

Schoene, D., Killmann, W., von Lüpke, H. \& Wilkie, M. (2007). Definitional Issues Related to Reducing Emissions from Deforesation in Developing Countries. Forests and Climate Change Workingpaper 5, Food and Agriculture Organization of the United Nations, Forestry Department, Rome.

Sheather, S. \& Jones, M. (1991). A reliable data-based bandwidth selection method for kernel density estimation. Journal of the Royal Statistical Society, 53(3), 683-690.

Sissenwine, N., Dubin, M. \& Wexler, H. (1962). The US Standard Atmosphere, 1962. 
Journal of Geophysical Research, 67(9), 3627-3630.

Skøien, J. O. \& Blöschl, J. (2006). Sampling scale effects in random fields and implications for environmental monitoring. Environmental monitoring and assessment, 114(1-3), 521552.

Smith, W. (2002). Forest inventory and analysis: a national inventory and monitoring program. Environmental Pollution, 116, 233-242.

Stehman, S. V. (2000). Practical Implications of Design-Based Sampling Inference for Thematic Map Accuracy Assessment. Remote Sensing of Environment, 72(1), 35-45.

Stehman, S. V. (2004). Sampling Design for Accuracy Assessment of Large-Area, LandCover Maps: Challenges and Future Directions. In R. Lunetta \& J. Lyon (Eds.), Remote Sensing and GIS Accuracy Assessment (pp. 13-27). CRC.

Stehman, S. V. \& Czaplewski, R. L. (1998). Design and Analysis for Thematic Map Accuracy Assessment: Fundamental Principles. Remote Sensing of Environment, 64(3), 331 - 344.

Stern, N. (2007). The Economics of Climate Change: The Stern Review. Cambridge University Press.

Strahler, A., Woodcock, C. \& Smith, J. (1986). On the nature of models in remote sensing. Remote Sensing of Environment, 20(2), 121-139.

Strobl, C., Boulesteix, A., Zeileis, A. \& Hothorn, T. (2007). Bias in random forest variable importance measures: Illustrations, sources and a solution. BMC bioinformatics, 8(1), 25.

Stupariu, M., Pătru-Stupariu, I. \& Cuculici, R. (2010). Geometric approaches to computing 3D-landscape metrics. Landscape Online.

Tobler, W. (1970). A computer movie simulating urban growth in the detroit region. Economoic Geography, 46, 223-240.

Tomppo, E., Schadauer, K., McRoberts, R., Geschwantner, T., Gabler, K. \& Ståhl, G. (2010). Introduction. In E. Tomppo, T. Gschwantner, M. Lawrence, \& R. McRoberts (Eds.), National Forest Inventories - Pathways for Common Reporting (pp. 1-18). Springer. 
Traub, B. \& Kleinn, C. (1999). Zur Erfassung von Flächenfragmentierung und struktureller Diversität. Forstwissenschaftliches Centralblatt, 118(1), 39-50.

Traub, B., Köhl, M., Paivinen, R. \& Kugler, O. (2000). Effects of different definitions on forest area estimation in national forest inventories in Europe. Unites States Department of Agriculture Forest Ecology and Management Forest Service Gerneral Technical Report, (pp. 176-184).

Tso, B. \& Mather, P. (2009). Classification methods for remotely sensed data. CRC Press, 2 edition.

Turner, M., O'Neill, R., Gardner, R. \& Milne, B. (1989). Effects of changing spatial scale on the analysis of landscape pattern. Landscape Ecology, 3(3), 153-162.

UN (1992). Multilateral Convention on biological diversity (with annexes). Concluded at Rio de Janeiro on 5 June 1992. In United Nations Treaty Series, volume 1760 (pp. 146). Rio de Janeiro: United Nations. Deutsche Übersetzung.

UNFCCC (2003). Modalities and procedures for afforestation and reforestation project activities under the clean development mechanism in the first commitment period of the kyoto protocol, fccc/cp/2003/6/add.2. In Report of the Conference of the Parties on its Ninth Session (pp.13). Milan: UN Framework Convention on Climate Change. Decision 19/CP.09.

VanDerWal, J., Falconi, L., Januchowski, S.and Shoo, L. \& Storlie, C. (2011). Species Distribution Modelling Tools: Tools for processing data associated with species distribution modelling exercises, 1.1-5 edition. R-Package.

Verchot, L., Zomer, R., Straaten, O. \& Muys, B. (2007). Implications of country-level decisions on the specification of crown cover in the definition of forests for land area eligible for afforestation and reforestation activities in the CDM. Climatic Change, 81(3), $415-430$.

Vermote, E., Tanré, D., Deuzé, J. L., Herman, M., Morcrette, J. J. \& Kotchenova, S. Y. (2006). Second Simulation of a Satellite Signal in the Solar Spectrum-Vector (6SV), 6s user guide 3 edition.

Vermote, E. F., Tanré, D., Deuze, J. L., Herman, M. \& Morcette, J. J. (1997). Second 
simulation of the satellite signal in the solar spectrum, 6s: An overview. Geoscience and Remote Sensing, IEEE Transactions on, 35(3), 675-686.

Vidal, C., Lanz, A., Tomppo, E., Schadauer, K., Gschwantner, T., di Cosmo, L. \& Robert, N. (2008). Establishing forest inventory reference definitions for forest and growing stock: a study towards common reporting. Silva Fennica, 42(2), 247.

Vierenklee, J. E. \& Meinert, F. (1797). Anfangsgründe der theoretisch-praktischen Arithmetik und Geometrie für diejenigen, welche sich dem Forstwesen widmen: Mit Kupfern und einem illuminirten Plane. Leipzig: Weidmann. Nach den gegenwärtigen Bedürfnissen verbessert und vermehrt von Friedrich Meinert.

Walker, W., Stickler, C., Kellndorfer, J., Kirsch, K. \& Nepstad, D. (2010). Large-Area classification and mapping of forest and land cover in the Brazilian Amazon: a comparative analysis of ALOS/PALSAR and Landsat data sources. Selected Topics in Applied Earth Observations and Remote Sensing, IEEE Journal of, 3(4), 594-604.

Walter, H., Lieth, H., Rehder, H. \& Harnickell, E. (1964). Klimadiagramm Weltatlas. G. Fischer.

Walz, U., Hoechstetter, S. \& Thinh, N. X. (2007). Daten und Methoden zur Analyse von dreidimensionalen Landschaftsstukturen. Photogrammetrie, Fernerkundung, Geoinformatik, 4, 225-238.

Walz, U., Hoechstetter, S., Vock, D. \& Dang, L. H. (2010). LandMetrics-3D Landscape metrics for raster-based structure analysis in three dimensions. Leibniz Institute of Ecological and Regional Development (IOER), Weberplatz 1, D-01217 Dresden, Germany. Software.

Wappes, L. (1893). Vergleichende Untersuchungen über die Waldeinteilung bei der Forsteinrichtung in einigen deutschen Staaten. Inaugural-dissertation, München, Phil. Fak., Langensalza.

Weiss, G. \& Provost, F. (2001). The effect of class distribution on classifier learning: an empirical study. Technical Report ML-TR-44, Department of Computer Science, Rutgers University.

Woodcock, C. \& Strahler, A. (1987). The factor of scale in remote sensing. Remote Sensing 
of Environment, 21(3), 311-332.

Wu, J. (2004). Effects of changing scale on landscape pattern analysis: scaling relations. Landscape Ecology, 19(2), 125-138.

Zarco-Tejada, P. J. \& Miller, J. R. (1999). Land cover mapping at BOREAS using red edge spectral parameters from CASI imagery. Journal of Geophysical Research, 104(D22), 921-933.

Zeide, B. (1980). Plot size optimization. Forest Science, 26(2), 251-257.

Zevenbergen, L. \& Thorne, C. (1987). Quantitative analysis of land surface topography. Earth surface processes and landforms, 12(1), 47-56.

Zheng, D., Heath, L., Ducey, M. \& Simth, J. (2009). Quantifying scaling effects on satellitederived forest area estimates for the conterminous usa. International Journal of Remote Sensing, 30(12), 3097-3114.

Zingg, A. \& Bachofen, H. (1988). Schweizerisches Landesforstinventar: Anleitung für die Erstaufnahme 1982-1986. Eidgenössische Anstalt für das forstliche Versuchswesen.

Zomer, R., Trabucco, A., Verchot, L. \& Muys, B. (2008). Land area eligible for afforestation and reforestation within the Clean Development Mechanism: a global analysis of the impact of forest definition. Mitigation and Adaptation Strategies for Global Change, 13(3), 219-239.

Zöhrer, F. (1980). Forstinventur. Hamburg, Berlin: Verlag Paul Paray. 

Teil VII.

Anhang 


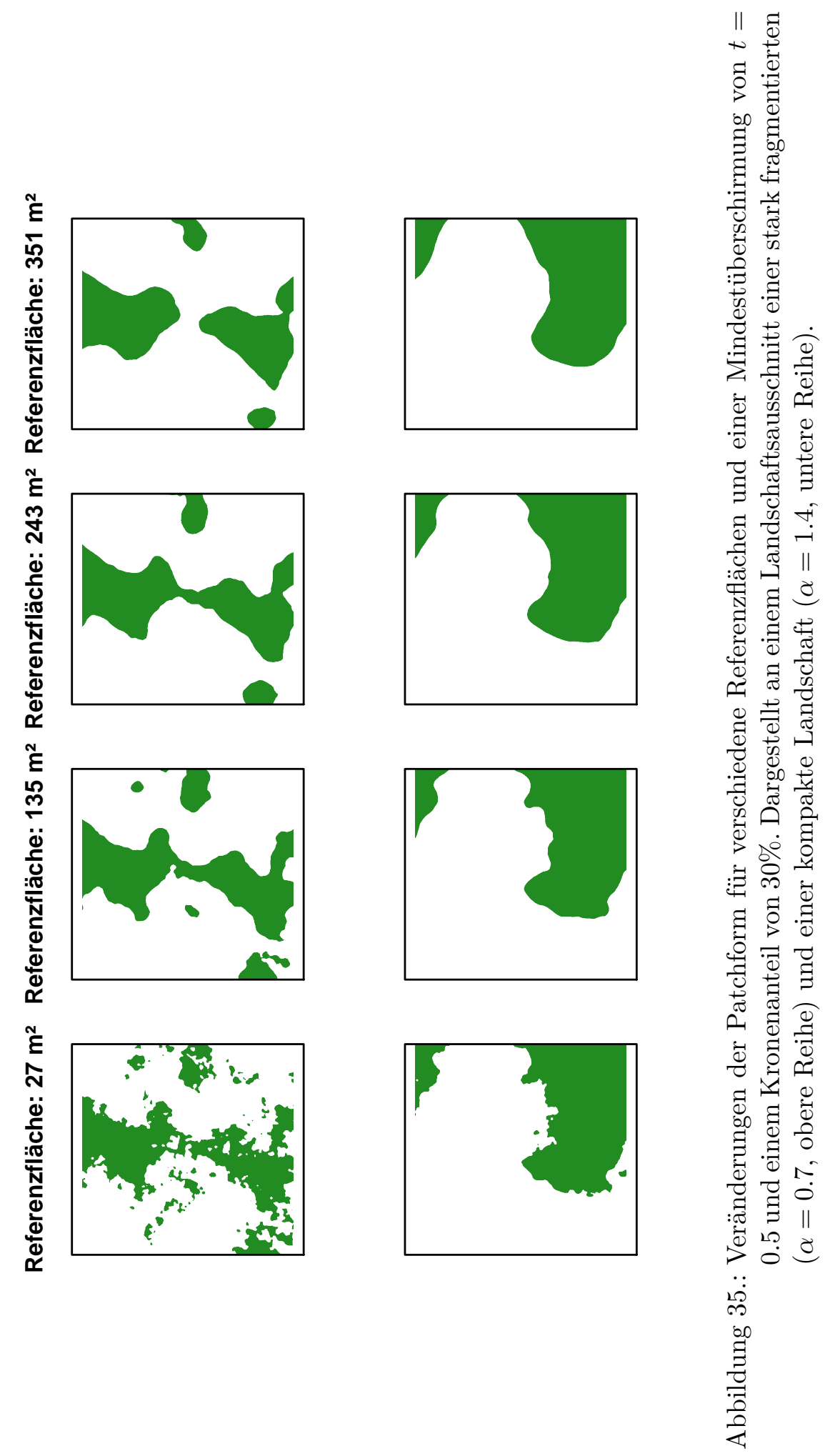


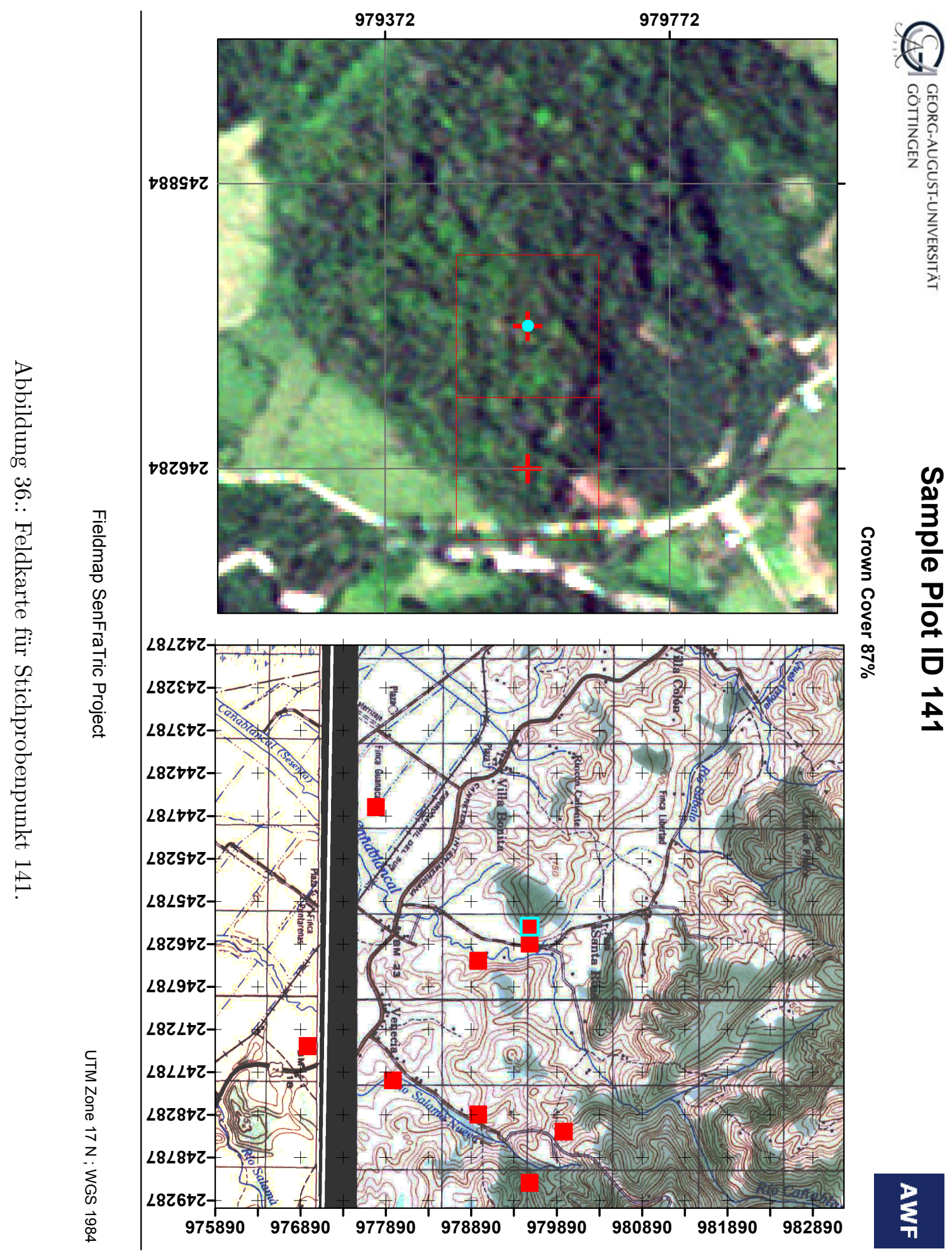

